\title{
Molecular characterization of non-subtype C and recombinant HIV-1 viruses from Cape Town, South Africa
}

\author{
by

\section{Eduan Wilkinson}

Submitted in fulfilment for the degree

MSc in BioMedical Science

at

\section{Stellenbosch University}

Division of Medical Virology, Department of Pathology

Faculty of Health Science

Supervisor: Prof Susan Engelbrecht

Date: 1 December 2008 


\section{Declaration}

$\mathrm{I}$, the undersigned, hereby declare that the work contained in this thesis is my own original work and that I have not previously in its entirety or in part, submitted it at any university for a degree.

Signature

Name in full

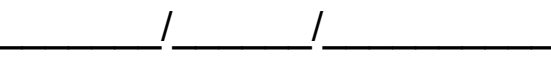

Date

Copyright (C) 2008 Stellenbosch University

All rights reserved 


\section{Acknowledgements}

I would hereby like to extend my fullest gratitude to the following people and institutions; without which this thesis would not have been possible:

Prof. Susan Engelbrecht, the promoter of this study, for her expert advice, guidance and assistance throughout the course of this project.

Annette Laten for all her assistance in the laboratory and with the sequences.

To my parents, Edward and Elizabeth Wilkinson, for always believing in me and supporting me with my studies.

To all the people in the Division of Medical Virology, Department of Pathology, Faculty of Health Sciences, University of Stellenbosch for their moral support throughout the course of the two years.

To my friends for all their support and encouragement throughout my studies.

To the Poliomyelitis Research Foundation (PRF) and the National Research Foundation (NRF) of South Africa for the generous bursary money.

To the Harry Crossly and the Poliomyelitis Research Foundation for funding the project. 


\begin{abstract}
HIV-1 was first diagnosed within South Africa in 1982. In the 1980's homosexual transmission dominated the HIV-1 epidemic within the country. In the late 1980's the second HIV-1 epidemic was recognized amongst heterosexual individuals. Today heterosexual transmission of HIV-1 dominates the epidemic in South Africa. Subtype C HIV-1 is responsible for the overwhelming majority of heterosexual infections. An estimated $95 \%$ of all infections in the country are thought to be subtype $C$ related. To date only a few papers have been published on non-subtype C HIV within the country. This study characterized subgenomic and near full-length sequences of nonsubtype C HIV-1 viruses from the Cape Town area.
\end{abstract}

The gag p24, pol-integrase, and env gp41 regions of 11 of the 12 samples were characterized by amplification and direct sequencing. Phylogenetic analysis of the sequenced data, with online subtyping tools (REGA and jpHMM) and the drawing of NJ-trees revealed the presence of subtype $A 1, B$, $\mathrm{F} 1$ and recombinant viral forms such as $A D, A G$ and $A C$. One of the isolates was classified as a subtype $\mathrm{C}$ and was included for control purposes.

Near full-length characterization of four of the samples were attempted, through full genome PCR amplification and sequencing. Analysis of sequenced data with the use of subtyping-, recombination identification, and tree drawing tools revealed a subtype $B$, and $A 1$ isolate. The other two isolates were identified as possible $A C$ and $A D$ recombinants.

The data that was generated will greatly improve our knowledge of nonsubtype $\mathrm{C}$ isolates circulating within South Africa. Due to the possible impact that the high degree of genetic variation that HIV may have on vaccine design and development and ARV treatment and HIV diagnosis, ongoing research of the epidemiology and spread of HIV within South Africa are needed. 


\section{Opsomming}

MIV was in 1982 vir die eerste keer in Suid Afrika gediagnoseer en was hoofsaaklik deur homoseksuele kontak oorgedra. Aan die begin van die 1990's is 'n tweede MIV epidemie gewaar onder heteroseksuele individue. Heteroseksuele oordrag van die virus domineer tans die MIV epidemie in Suid Afrika en is meestal subtipe C verwant. Subtipe C, MIV-1 is verantwoordelik vir 95 persent van alle infeksies in die land. Tot hede is slegs 'n paar publikasies oor die nie-subtipe $C$ epidemie in die land gepubliseer. Die huidige studie was gemik op die karakterisering van subgenomiese en vollengte genome van nie-subtipe C MIV isolate van die Kaapstad omgewing.

Die gag p24, pol-integrase en env gp41 subgenomiese fragmente van 12 monsters was gekarakteriseer deur amplifikasie en DNS nukleotied volgorde bepaling. Filogenetiese analise deur middel van subtipering (REGA en jpHMM aanlyn subtiperings programme) asook NJ-filogenetiese bome van die data het die teenwoordigheid van subtipe $A 1, B$, en $F 1$, asook verskeie rekombinante viruse insluitende $A G, A D$ en $A C$ vorme aangedui. Een van die isolate was geklassifiseer as 'n subtipe C maar is in die studie ingevoeg vir kontrole doeleindes.

Vollengte karakterisering van 4 uit die 12 isolate was ook gedoen deur vollengte genoom amplifikasie en DNS nukleotied volgorde bepaling. Tydens die analisering van die DNS volgorde data, deur middel van aanlyn subtipering, rekombinasie identifikasie (Simplot en RIP), en filogenetiese boom konstruksie programme is twee isolate geidentifiseer as subtipe $B$ en A1 MIV-1 viruse. Die ander twee isolate was as moontlike $A C$ en $A D$ rekombinante geklassifiseer.

Die data van nie-subtipe C MIV isolate sal ons kennis van die nie-subtipe C epidemie in Suid Afrika versterk. As gevolg van die impak wat die hoë graad van genetisie variasie van MIV op die ontwikkeling van entstowwe, sowel as die diagnose en behandeling van pasiente kan hê, is verdere navorsing in die epidemiologie van die MI-virus in Suid Afrika nodig. 


\section{Table of Contents}

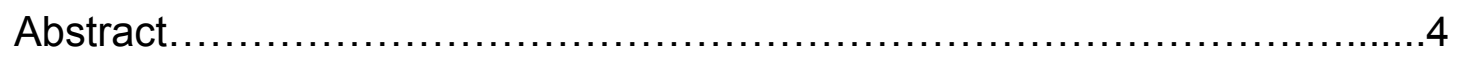

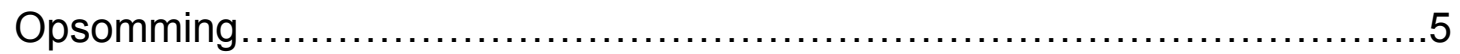

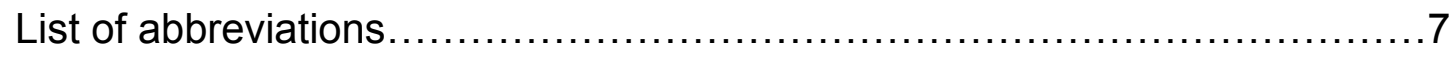

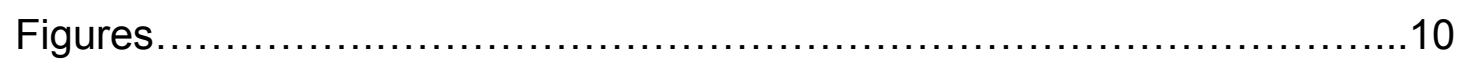

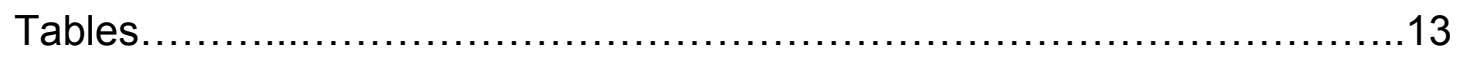

Chapter 1: INTRODUCTION AND LITERATURE REVIEW 15

$\begin{array}{ll}\text { INTRODUCTION } & 16\end{array}$

$\begin{array}{ll}\text { LITERATURE REVIEW } & 17\end{array}$

$\begin{array}{ll}\text { AIM OF THE STUDY } & 50\end{array}$

Chapter 2: MATERIALS AND METHODS 51

$\begin{array}{ll}\text { Chapter 3: RESULTS } & 76\end{array}$

Chapter 4: DISCUSSION AND CONCLUSION 105

$\begin{array}{ll}\text { DISCUSSION } & 106\end{array}$

$\begin{array}{ll}\text { CONCLUSION } & 115\end{array}$

$\begin{array}{lr}\text { Chapter 5: REFERENCES } & 116\end{array}$

$\begin{array}{ll}\text { Chapter 6: APPENDIX } & 145\end{array}$ 


\section{List of abbreviations}

${ }^{\circ} \mathrm{C}$ - degrees Celsius

$\mu \mathrm{l}$ - micro liters

A - Adenine

AIDS - Acquired Immunodeficiency syndrome

ARV - Antiretroviral

BEAST - Bayesian Evolutionary Analysis Sampling Trees

BLAST - Basic Local Alignment Search Tool

bp - base pairs

C - Cytosine

CA - California

CCR5 - Chemokine (C-C motif) receptor 5

CD4 - cluster of differentiation 4

CDC - Center for Disease Control and Prevention

cDNA - complimentary deoxyribonucleic acid

CRF - Circulating recombinant form

CTL - Cytotoxic T Iymphocytes

DDBJ - DNA Database of Japan

DNA - Deoxyribonucleic acid

dNTP - Deoxyribonucleotide triphosphates

DOE - Department of Energy

DRC - Democratic Republic of the Congo

EMBL - European Molecular Biology Laboratory

F - Forward Primer

g- Gravitational constant

G - Guanine

gp - glycoprotein

GTR - General Time Reversible Model

HIV - Human Immunodeficiency Virus

HIV-1 - Human Immunodeficiency Virus type 1

HIV-2 - Human Immunodeficiency Virus type 2

HKY - Hasegawa, Kishino, Yano Model 
HMA - Hetroduplex Mobility Assay

HSRV - Human Spuma Retrovirus

HTLV-I - Human T-cell leukemia virus type I

HTLV-II - Human T-cell leukemia virus type II

HTLV-III - Human T-cell leukemia virus type III

JAMA - Journal of American Medical Assosiation

jpHMM - jumping profile Hidden Markov Model

kbp - kilo base pairs

KS - Kaposi's sarcoma

LANL - Los Alamos National Laboratory

LAS - Lymphadenopathy syndrome

LAV - Lymphadenopathy virus

LAV-2 - Lymphadenopathy virus type 2

LTR - Long Terminal Repeats

$\mathrm{M}$ - Molecular marker

MEGA - Molecular Evolutionary Genetics Analysis

$\mathrm{MgCl}_{2}-$ Magnesium Chloride

MHC - Major Histocompatibility Complex

$\mathrm{mM}$ - millimoles

MMWR - Morbitity and Mortality Weekly

mRNA - messenger RNA

NCBI - National Center for Biolnformatics

NFLG - Near full-length genome

NIAID -National Institute of Allergy and Infectious Diseases

$\mathrm{nm}$ - nanometers

$\mathrm{NM}$ - New Mexico

$\mathrm{NNI}$ - Nearest Neighbor Interchange

NRF - National Research Foundation

NY - New York

OTU - Operating Taxonomic Units

$\mathrm{p}$ - Protein

PAUP - Phylogenetic analysis using Parsimony* and other methods

PBMC - Peripheral Blood Mononuclear Cell

PCP - Pneumocystis carinii pneumonia 


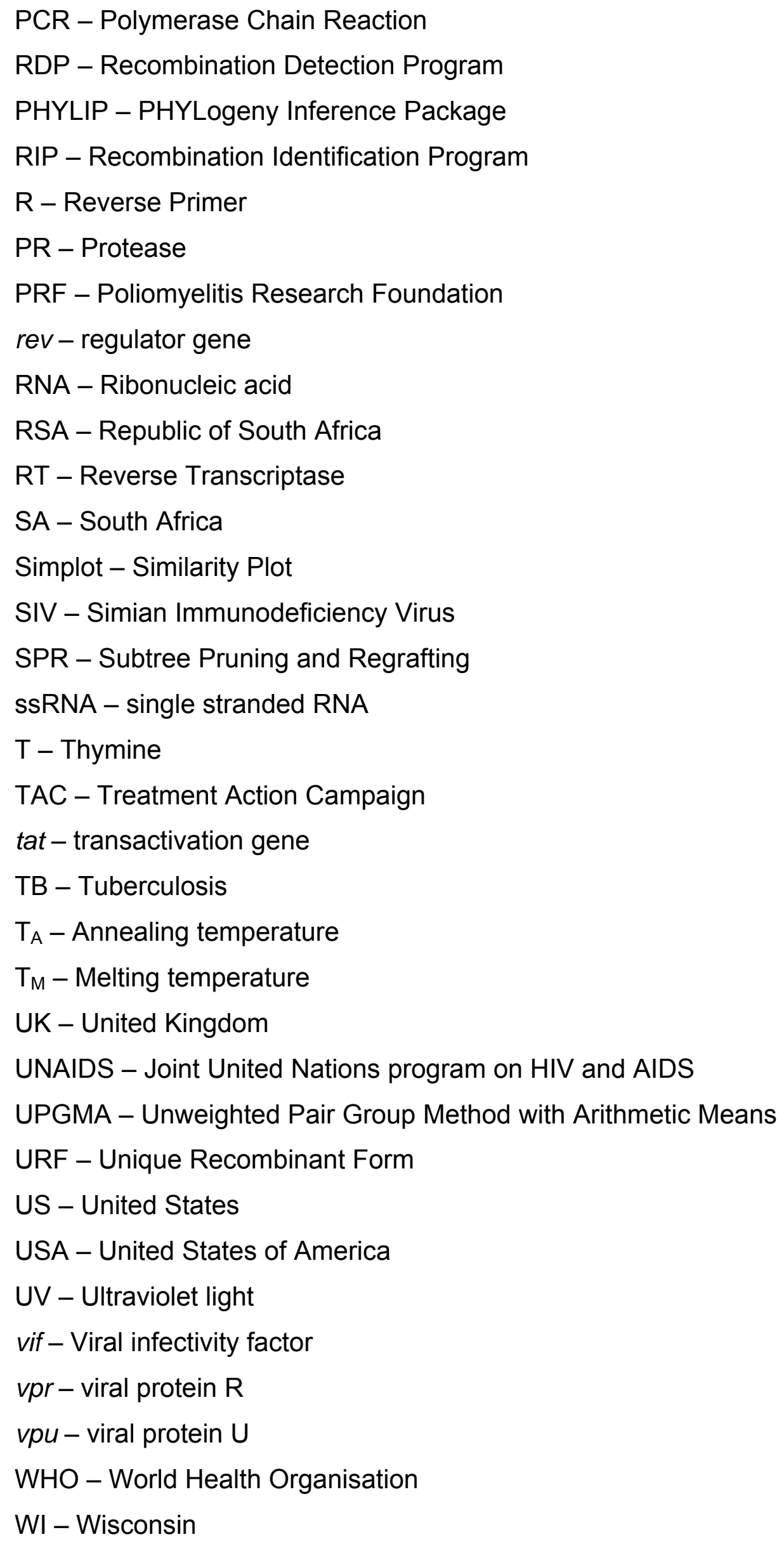




\section{List of Figures}

Page

Figure 1.1: A phylogenetic tree depicting the evolutionary

relationship between: HIV-1 and its major groups;

HIV-2; and several different SI-viruses isolated from

different primates on the African continent. 21

Figure 1.2: A schematic diagram of the HIV virion. 24

Figure 1.3: $\quad$ A diagrammatical representation of the genome layout of HIV-1. 25

Figure 1.4: $\quad$ The HIV-1 life cycle. 27

Figure 1.5: $\quad$ Creation of a URF in a coinfected individual. 31

Figure 1.6: $\quad$ Global distribution of different HIV-1 subtypes and circulating recombinant forms. 32

Figure 1.7: The genomic structure of a CRF01_AE isolate. 34

Figure 1.8: $\quad$ Subtype distribution of HIV-1 in a handful of sub-Sahara African countries. $\quad 35$

Figure 1.9: The genomic structure of CRF02_AG. 36

Figure 1.10: $\quad$ A simplistic representation of a phylogenetic tree. 47

Figure 1.11: $\quad$ A graphical representation of a full-length amplification assay for the characterization of a near full-length HIV-1 genome. 49

Figure 2.1: $\quad$ A flow diagram summarizing the methodology used in the study.

Figure 2.2: $\quad$ Schematic diagrams of the full genome amplification of an HIV isolate using a long PCR amplification assay.

Figure 2.3: $\quad$ Schematic diagrams of the full genome amplification of an HIV isolate in four overlapping fragments. 
Figure 3.1: $\quad$ Agarose gel electrophoresis of the nested pol-integrase PCR products.

Figure 3.2: $\quad$ A Neighbor-joining tree of gag sequences (485 bp) indicating reference sequences and TV sequences. 82

Figure 3.3: $\quad$ A Neighbor-joining tree of pol sequences (944 bp) indicating reference sequences and TV sequences. 83

Figure 3.4: $\quad$ A Neighbor-joining tree of env sequences (438 bp) indicating reference sequences and TV sequences. 84

Figure 3.5: $\quad$ Agarose gel of the $9.2 \mathrm{kbp}$ PCR products of sample R84.

Figure 3.6:

A Neighbor-joining tree containing the NFLG sequences of the four TV samples and reference samples.

Figure 3.7: $\quad$ A Neighbor-joining tree containing reference sequences and the sequence of the gag-pol fragment of TV239.

Figure 3.8: $\quad$ A Neighbor-joining tree containing reference sequences and the sequence of the env-nef fragment of TV239.

Figure 3.9: $\quad$ The analysis of the TV239 env-nef fragment with RIP.

Figure 3.10:

The analysis of TV412 with RIP.

Figure 3.13 A: A schematic diagram of viral recombination within the TV239 env-nef fragment.

Figure 3.13 B: $\quad$ NJ-trees of the different viral recombinant regions of the TV 239 env-nef fragment.

Figure 3.14 A: A schematic diagram of viral recombination within sample TV412.

Figure 3.14 B: $\quad$ NJ-trees of the different viral recombinant regions of sample TV412. 
Page

Figure 3.15: $\quad$ A NJ tree exploring the relationship of subtype $B$ HIV-1 isolates with sample R84.

Figure 3.16: $\quad$ A Neighbor-joining tree looking at the relationship between subtypes A1 HIV-1 isolates and sample TV314.

Figure 6.1: $\quad$ REGA subtyping results of the gag p24 sequences.

Figure 6.2: $\quad$ REGA subtyping results of the pol - integrase sequences.

Figure 6.3: $\quad$ REGA subtyping results of the env gp41 sequences.

Figure 6.4:

REGA subtyping results of NFLG's fragments. 


\section{List of Tables}

Page

Table 1.1: $\quad$ The HIV-1 genes and proteins with their functions. 26

Table 1.2: Summary of published data of subgenomic fragments of HIV-1 isolates from South Africa.

Table 1.3: $\quad$ Published data of near-full length HIV-1 sequences from South Africa.

Table 2.1: $\quad$ Equipment used to perform sample analysis.

Table 2.2: $\quad$ List of chemicals and commercial products used in the study.

Table 2.3:

Software packages used in the analysis of sequenced data.

Table 2.4:

Patient samples and demographics.

Table 2.5:

List of different primers used in the amplification of the gag (p24), pol-integrase and the env (gp41) fragments.

Table 2.6: $\quad$ List of different sequencing primers for the gag p24, pol-integrase and the env gp41 fragments.

Table 2.7: $\quad$ Primers that were used for the amplification of $9.2 \mathrm{kbp}$ fragments

Table 2.8: $\quad$ Primers that were used for the four the overlapping fragments

(LTR-gag, gag-pol, pol-env, and env-LTR)

Table 2.9: PCR cycling condition of the four overlapping fragments.

Table 3.1

PCR amplification of the subgenomic regions of the 12 samples.

Table 3.2:

Sequencing results for the subgenomic regions.

Table 3.3: Subtyping analysis performed on the gag p24, pol-integrase and env gp41 fragments. 
Table 3.4: $\quad$ PCR amplification of the four overlapping fragments. 86

$\begin{array}{lll}\text { Table 3.5: } & \text { jpHMM and REGA subtyping tools. }\end{array}$

Table 3.6: $\quad$ Breakpoint coordinates of the four samples as was determined by jpHMM analysis. 96

Table 6.1: $\quad$ jpHMM subtyping results of gag p24 subgenomic regions.

Table 6.2: $\quad$ jpHMM subtyping results of $p o l$ - integrase subgenomic regions. 157

Table 6.3: $\quad$ jpHMM subtyping results of env gp41 subgenomic regions.

Table 6.4: $\quad$ Sequencing primers used for the characterization of R84.

Table 6.5: $\quad$ Sequencing primers used for the sequencing of sample TV239's.

Table 6.6: $\quad$ Sequencing primers used for the sequencing of TV314.

Table 6.7: $\quad$ Sequencing primers used for the sequencing of TV 412.

$165 / 166$

Table 6.8:

jpHMM subtyping results of NFLG's fragments.

208 


\section{CHAPTER ONE - Introduction and Literature Review}

Table of Content

Page

INTRODUCTION. 16

LITERATURE REVIEW

1.1 History. 17

1.1.1 The HIV pandemic...............................................

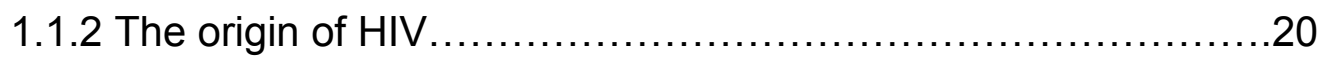

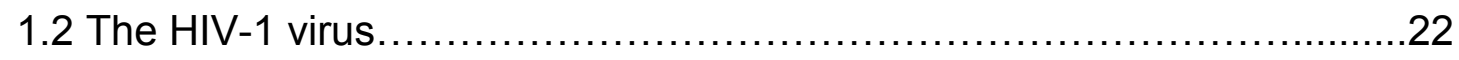

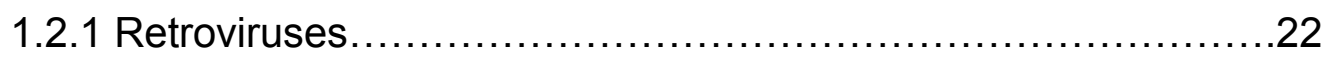

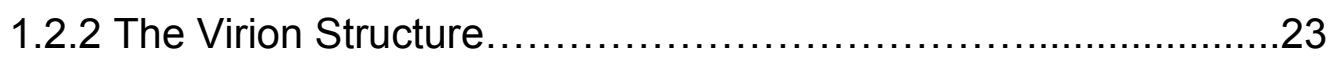

1.2.3 Genomic organization of HIV-1 ..................................

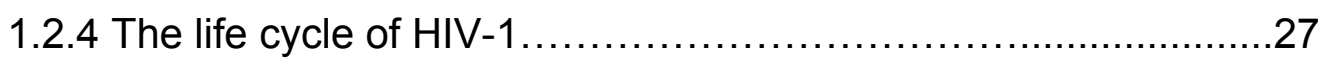

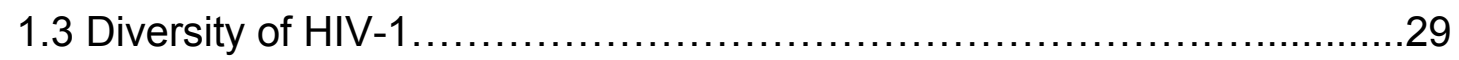

1.3.1 Global diversity of HIV-1 ....................................... 31

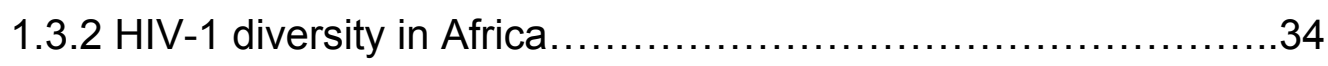

1.3.3 HIV-1 diversity in South Africa........................................36

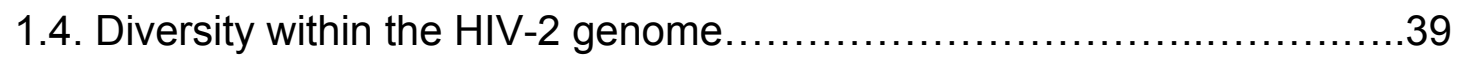

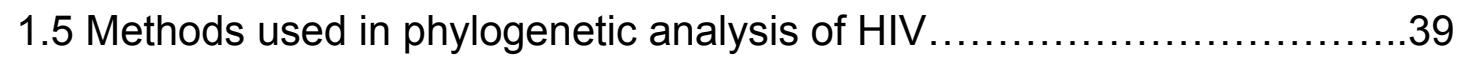

1.5.1 Searching for homologous sequences...........................40

1.5.2 Aligning homologues sequences................................... 41

1.5.3 Choosing a model of evolution...................................42

1.5.4 Drawing phylogenetic trees.......................................44

1.5.5 Detection of recombinant viruses.............................46

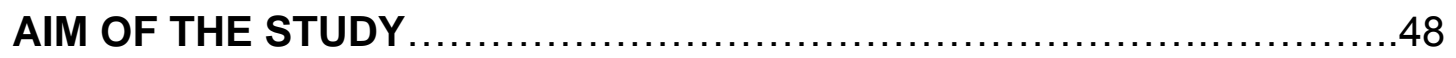




\section{CHAPTER ONE}

\section{INTRODUCTION}

The clinical condition known as acquired immunodeficiency syndrome (AIDS) is caused by the human immunodeficiency virus (HIV). The virus exists in two distinct forms, HIV-type 1 (HIV-1) and HIV-type 2 (HIV-2). With the help of epidemiological and molecular tools closer analysis has revealed that HIV-1 has spread all over the world, whereas HIV-2 is mostly confined to areas of West Africa [Essex and Mboup, 2002; Levy, 2007]. HIV-1 can be divided into three groups: group $\mathrm{M}$ (major), group $\mathrm{N}$ (non-M or non-O) and group $\mathrm{O}$ (outlier) [Peeters, 2001]. Group M HIV-1 is responsible for the majority of infections worldwide and can be subdivided into a large variety of subtypes and circulating recombinant forms (CRF's) [http://www.hiv.lanl.gov/]. The genetic similarity between HIV-1 and other closely related viruses, such as HIV-2 and SIV, can be used as genetic markers in the study of the virus with the help of molecular and phylogenetic techniques.

The July 2008 UNAIDS report, estimates that 34 million adults and 2.3 million children are living with HIVIAIDS, with 2.7 million new infections each year. The report also estimates that 2 million people die, due to AIDS related deaths each year [UNAIDS, 2008]. Although HIV and AIDS are found in all parts of the world, some areas are more severely affected than others. Of the 36 million people infected worldwide, an estimated 24.1 million infected individuals live in sub-Saharan Africa where, in some places, more than one in five adults can be infected [UNAIDS, 2008]. Though sub-Saharan Africa is most severally affected, the epidemic is spreading more rapidly in Eastern Europe and Central Asia, where the rate of new infections increased by as much as 50\% between 2004 and 2008 [UNAIDS, 2008].

In South Africa (SA) the HIV-I epidemic was initially associated with the homosexual population [Sher, 1989]. In the 1980's, HIV-1 was isolated form homosexual men, who introduced the virus into SA from other countries [Becker et al, 1985]. These initial isolates were later identified as HIV-1 
subtype B and D viruses [Becker et al, 1995; Engelbrecht et al, 1995]. Today the HIV-1 epidemic has spread widely amongst the heterosexual population of the region with as many as 6.2 million infected people in SA alone [UNAIDS, 2008]. Subtype C HIV-1 is responsible for nearly $52 \%$ of HIV infections worldwide and is commonly found in parts of the Indian subcontinent and eastern and southern Africa. In SA, it can account for as much as $95 \%$ of all infections [Ariën et al, 2007]. Though HIV-I subtype C still holds a dominant position within southern Africa, non-subtype C HIV-I infections have been growing in importance over the past couple of years, which may impact a wide spectrum of fields e.g. antiretroviral treatment [Spira et al, 2003], diagnostics and the development of an effective vaccine [Buonaguro et al, 2007; Peeters et al, 2003]. In the following section the history of the HIV pandemic, the genetic diversity, the virus structure, as well as phylogenetic methods used in analysis of HIV will be reviewed.

\section{LITERATURE REVIEW}

\subsection{History}

\subsubsection{The HIV pandemic}

Kaposi's Sarcoma (KS) was a very rare form of a relatively benign cancer that mostly affected the elderly [Gange and Jones, 1978; Penn, 1979]. By March 1981 eight cases of a more aggressive form of KS was reported amongst young homosexual men from New York [Hymes et al, 1981, Friedman-Kien et al, 1981]. At this time there were increases all over the United States (US) in the number of cases of a rare lung infection, Pneumocystis carinii pneumonia (PCP) [Gottlieb et al, 1981; Masur et al, 1981]. In April of 1981 these increases were noticed by a young drug technician working at the Center of Disease Control and Prevention (CDC) in Atlanta, when a doctor treating a young patient with $\mathrm{PCP}$ requested a refill for the drug used in the treatment of PCP patients [CDC, 1981]. This was very unusual as most patients responded to medication within 10 days of treatment or they passed away. Afterwards a number of theories were developed about the possible cause of these opportunistic infections and cancers. 
By the end of 1981 it was clear that the disease was affecting other population groups, when the first cases of PCP infection were reported in intravenous drug users [Shilts, 1987]. Shortly afterwards, the first reported cases of these opportunistic infections were documented in continental Europe and the UK. By the beginning of the next year the disease still did not have a name. By June 1982 a report suggested that the disease might be caused by an infectious agent that was sexually transmitted, after a small cluster of cases amongst homosexual men in southern California was reported. By October 1982, 452 cases from 23 states had been reported to the CDC [CDC, 1982]. It soon became apparent that the disease was also occurring amongst heterosexuals. By this time reported cases had been observed in homosexual and heterosexual people, intravenous drug users, certain blood transfusion recipients [Curran et al, 1984; Jaffe et al, 1984], some organ transplant recipients [Curran et al, 1984], a few newborn infants [Rubinstein et al, 1983; Oleske et al, 1983] and international travelers from African decent [Clumeck et al, 1983].

The acronym AIDS was beginning to be used on government level, the press and in scientific journals. Doctors thought AIDS was an appropriate name because people acquired the condition rather than inherited it, resulting in a deficiency within the immune system, with a number of manifestations. By this time still very little was known about the transmission of the infectious agent and public anxiety started to grow. By the end of 1982 a 20-month old child who received multiple transfusions of blood and blood products died from infections related to AIDS [Curran et al, 1984]. Meanwhile in Uganda, doctors were beginning to see the first cases of a new, fatal wasting disease which came to be known locally as "slimming-disease" [Serwadda et al, 1985].

In May 1983 doctors and scientist at the Pasteur Institute in France reported the isolation of a new retrovirus from the lymph node of a man with persistent Lymphadenopathy Syndrome (LAS), which they suggested might be the causative agent of AIDS and was subsequently named Lymphadenopathy virus (LAV) [Barre-Sinoussi et al, 1983]. At the same time, reports from Europe suggested that two rather separate AIDS epidemics were occurring. In 
the UK, Germany and Denmark, the majority of people with AIDS were homosexual men with a history of sexual encounters with American nationals. In France and Belgium however, AIDS occurred mainly in migrants from former African colonies or those with links to areas in Africa [Clumeck et al, 1983]. Due to the fact that these patients had no history of blood transfusion, homosexuality or intravenous drug use, European and American scientists set out to discover more about the occurrence of AIDS in Africa [Weller et al, 1984]. By the end of 1983 the World Health Organization (WHO) reported that the disease were present in the United States, Canada, fifteen European countries, Haiti, Zaire (now the Democratic Republic of the Congo), seven Latin American countries, Australia and Japan [WHO, 1983].

A year after the team of Barre-Sinoussi [Barre-Sinoussi et al, 1983] isolated the Lymphadenopathy virus at the Pasteur Institute, Robert Gallo and his colleagues postulated that a variant T-lymphotropic retrovirus, which they called HTLV-III, might be the causative agent of AIDS [Gallo et al, 1984]. By 1984, Levy and co-workers, found the same viral agent that Barre-Sinoussi [Barre-Sinoussi et al, 1983] discovered an year earlier in one of the samples of an infected patient [Levy et al, 1984]. Ratner and co-workers independently confirmed that the new variant of HTLV-III was the causative agent of AIDS and also published the first full sequenced genome of the virus [Ratner et al, 1985a; Ratner et al, 1985b]. Medical data and records of patients suffering from opportunistic infections, from Central African countries, suggested that the disease was already present within the region in the 1970's and asserted the claims that it might have arisen from the region [Quinn et al, 1986]. Though HIVIAIDS was initially associated with people from particular risk groups, such as homosexuals and intravenous drug users, heterosexual transmission is now responsible for the majority of new infections [Osmanov et al, 2002; Esparza and Bhamaraparvati, 2000].

By 1986 two new retroviruses were isolated from patients with AIDS like symptoms from West Africa. Closer analysis of these viruses (LAV-2 or later called HIV-2), showed that although both caused immunodeficiency and AIDS in infected individuals, HIV-1 and HIV-2 differ in their natural history of 
infection and pathogenicity [De Cock et al, 1993; Pepin et al, 1991]. Since then it has been shown that both HIV-1 and HIV-2 shares structural, genetic and biological properties and cause CD4 cell depletion in infected individuals [Markovitz, 1993].

\subsubsection{The origin of HIV}

HIV is a member of the Lentivirus subfamily of retroviruses (Retroviridae). Since HIV was established as the etiological agent of AIDS an estimated 60 million people have been infected with HIV-1 worldwide [UNAIDS, 2008]. Though HIVIAIDS only came under the attention of humans in the early 1980's recent scientific discoveries dates the existence of the virus in humans as far back as the 1930's [Hahn et al, 2000; Korber et al, 2000]. A plasma sample from 1959 that was taken from a patient from the Central African country of Zairë (now the DRC), gives credible evidence that the disease has been in humans for some time longer than we first thought and also suggests that the epidemic might have originated in Africa [Nahmias et al, 1986].

Considerable evidence for a simian ancestor for HIV exists today. Simian immunodeficiency virus (SIV), which is also a member of the lentivirus family, is found in a large group of species of non-human primates and is related to HIV on a genomic level (Figure 1.1) [Myers et al, 1992]. All factors indicate that HIV originated through cross-species transmission from naturally infected primates to humans in Africa, a process commonly known as zoonosis [Hahn et al, 2000]. Phylogenetic analysis indicated that multiple zoonotic events, from simian species to humans, lead to the formation of two genetically distinct types of HIV (HIV-1 and HIV-2) and three main groups of HIV-1 [Gao et al, 1999; Papathanasopoulos et al, 2003a].

Molecular studies showed that HIV-1 is more closely related to primate lentiviruses from chimpanzees $\left(\mathrm{SIV}_{\mathrm{cpz}}\right)$, mainly from the subspecies Pan troglodytes troglodytes [Gao et al, 1999]. Similarly HIV-2 is more closely related to SIV sequences commonly found in sooty mangabeys, Caeoceus atys, [Gao et al, 1992; Hirsch et al, 1989]. Chimpanzees and other non-human primates are commonly hunted for food in certain regions in Central and 
Western Africa and represent an easy source for zoonotic transmission of SIV to humans [Hahn et al, 2000; Papathanasopoulos et al, 2003a; Essex and Kanki, 1998]. Sooty mangabeys are also commonly used as a food source and in some cases domesticated as house pets in parts of Western Africa [Peeters et al, 2003]. Further support for the zoonotic infections of humans is that natural SIV infections in their simian hosts fail to induce a state of disease, which indicates that the virus has adapted itself to the host or that they co-exist in a symbiotic way [Cichutek and Norley, 1993; Rey-Cuille et al, 1998; Silvestri et al, 2003].

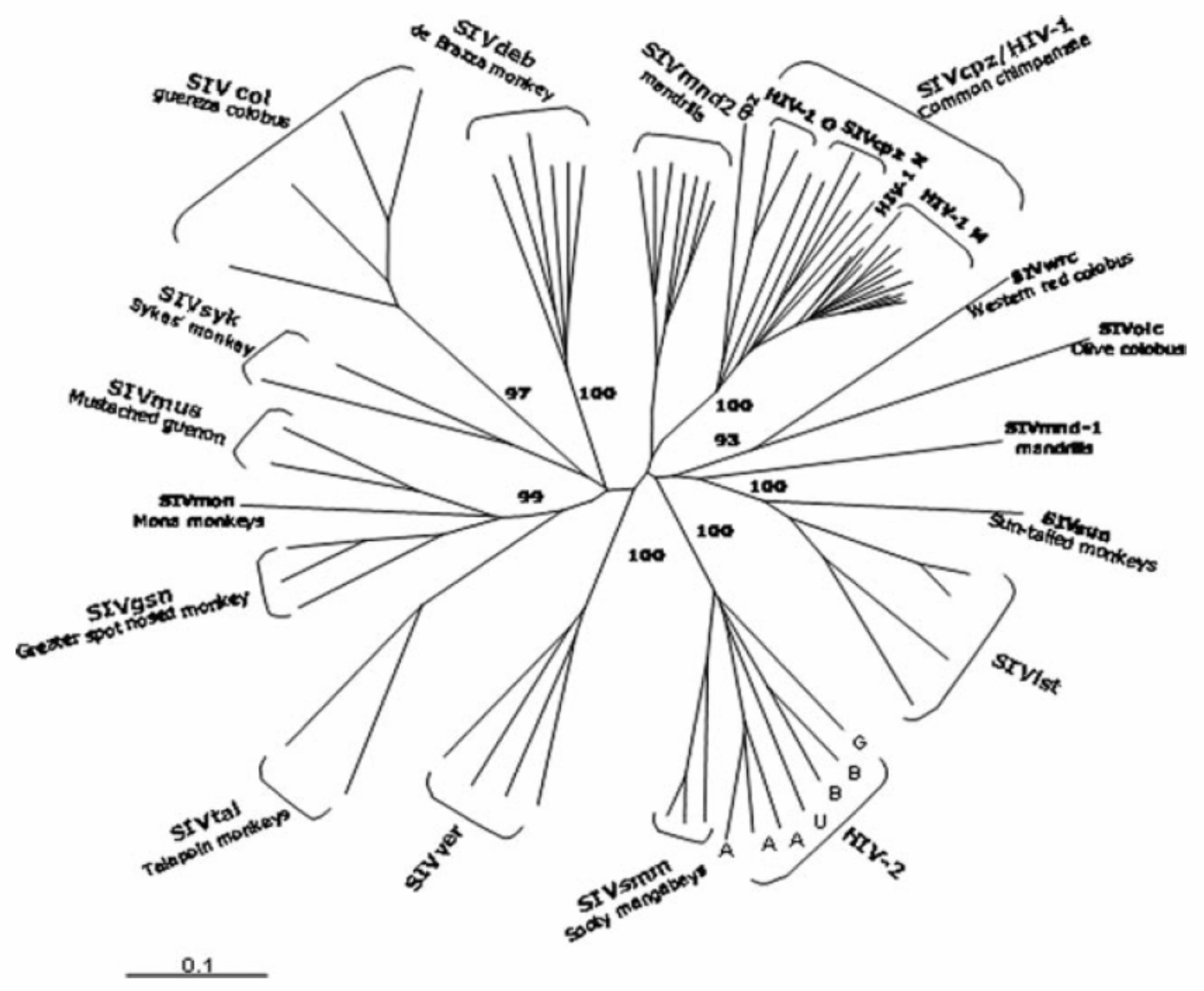

Figure 1.1: A phylogenetic tree depicting the evolutionary relationship between: HIV-1 and its major groups; HIV-2; and several different SI-viruses isolated from different primates on the African continent. Bootstrap values greater than 90 percent are included in the tree [Adapted from Buonaguro et al, 2007].

The timing of the zoonotic events leading to the rise of HIV in man has been a question for debate. Advanced phylogenetic methods have estimated 1930 as the time of the last common ancestor of the HIV-1 group of viruses [Korber et 
al, 2000; Salemi et al, 2001]. These estimations are based on the assumption of a molecular clock, with genetic change in a linear function and substitution occurring according to a Poisson distribution. Similarly the best estimate of the most recent ancestor of the HIV-2 group was estimated to be 1940 for HIV-2 group A and 1945 for HIV-2 group B, plus or minus 16 years [Lemey et al, 2003].

\subsection{The HIV-1 virus}

\subsubsection{Retroviruses}

The first discovery of a retrovirus was made as far back as 1910 at the Rockefeller Institute of Medical Research in New York. It was called avian sarcoma virus and induced tumors in muscle, bone and other tissues of chickens [Huebner and Todaro, 1969]. Later other retroviruses were identified that could induce the same symptoms in mice and other mammals [Essex et al, 1975; Hardy et al, 1973]. Retroviruses possess a unique enzyme called reverse transcriptase (RT), which uses the viral RNA as a template for making a DNA copy, which is then integrated into the host cell nucleic acid [Bebenek et al, 1989; Boyer et al, 1992]. The discovery of reverse transcriptase were profound in the field of biology as it overturned the central belief of molecular biology, that genetic information flows only in one direction (which is from DNA to RNA and on to protein synthesis). Retroviruses are also diploid, which means that there is a constant opportunity for recombination to occur when different parental genomes are packaged in the same virus particle [Robertson et al, 1995]. Initially these retroviruses were regarded to be of little importance because they were only associated with organisms other than humans. That all changed with the discovery of human associated retroviruses.

To date several different human retroviruses have been identified which can be divided into three families including; Lentivirinae (HIV-1 and HIV-2), Oncovirinae (HTLV-I and HTLV-II) and Spumavirinae. The human spumavirus (HSRV) was the first isolated human retrovirus [Achong et al, 1971]. Spumaviruses are found worldwide and can be isolated from a wide range of 
species, from monkeys to cattle [Flügel, 1991]. To date no known form of pathology of HSRV in humans has been found. The Oncovirinae family all shares the ability to induce cancerous conditions in its hosts, including lymphomas, leukemia, carcinoma and other forms of cancer. Two species of this family of retroviruses have been identified in humans to date, HTLV-I and HTLV-II. In 1976 a retrovirus was isolated from the lymph node of an ATLpatient (adult T-cell leukemia-lymphoma patient) in Japan [Uchiyama et al, 1977]. The etiological agent of ATL was named human T-cell leukemia virus Type - I or HTLV-I [Ammann et al, 1983; Fahey et al, 1984]. HTLV-I proved to be endemic in Japan, Central and South America, the Caribbean and Africa. The origin and the transmission of HTLV-I are thought to be the same as for HIV-1. Later a second virus, HTLV-II was isolated from the cells of a patient with a rare form of leukemia [Gallo, 2005]. An Africa origin was later hypothesized for HTLV-II, which then spread to other areas of the world where it is mostly associated with intravenous drug users [Vandamme et al, 1998]. The Lentivirinae family of retroviruses includes HIV-1, HIV-2 and other lentiviruses commonly found in non-human primates which are termed SIV. Later the fifth human retrovirus, HIV-2, was isolated from mildly immune suppressed patients in West Africa which appeared to be less pathogenic [Marlink et al, 1994; Kanki et al, 1994].

\subsubsection{The Virion Structure}

HIV-1 and HIV-2 belong to the group of lentiviruses [http://www.hiv.lanl.gov/] which can be distinguished from other retroviruses (such as HTLV-I and -II) by the presence of a cone-shaped nucleoid, absence of oncogenicity and the length and slow onset of clinical symptoms. The HIV particle is approximately $100 \mathrm{~nm}$ in size with an outer envelope of a lipid bilayer, which arises from the virus budding from the host cell. This lipid bilayer is penetrated by 72 glycoprotein spikes, the envelope (env) protein. The env polypeptide is composed of two subunits: the outer glycoprotein knob (gp120) and a transmembrane portion (gp41) which connects the knob to the virus lipid envelope [Levy, 2007] (Figure 1.2). 
On the inside of the lipid bilayer the envelope is lined with a matrix protein (p17). Also present within the lipid envelope are cellular proteins such as $\mathrm{MHC}$ class 1 and class 2 antigens. In HIV-1 the lipid envelope encloses an icosahedral shell of protein (p24) which in turn encloses a cone-shaped protein core ( $\mathrm{p} 7$ and p9 proteins). Within the cone-shaped core are two molecules of ssRNA in the form of a ribonucleoprotein. Bound to the diploid, positive-sense ssRNA are multiple copies of reverse transcriptase (RT), integrase (for genome integration into the host cell nucleic acid) and protease enzymes [Levy, 2007].

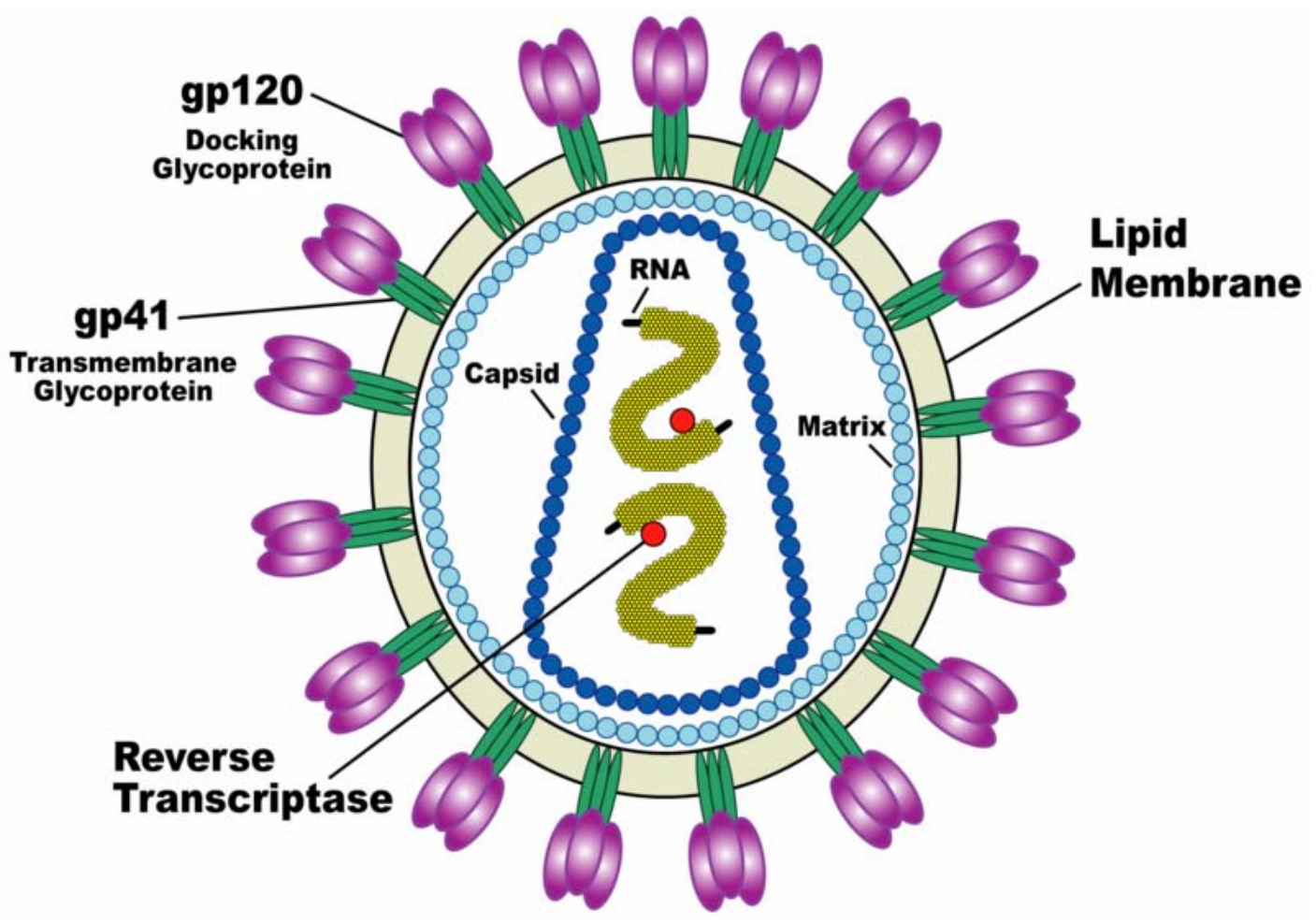

Figure 1.2: A schematic diagram of the HIV virion. The diagram displays all the major proteins and the ssRNA [http://en.wikipedia.org/wiki/HIV].

\subsubsection{Genomic organization of HIV-1}

The genome of the HIV-1 virus is approximately $9.7 \mathrm{~kb}$ long with long terminal repeats (LTR's) on both sides (Figure 1.3). Within the $9.7 \mathrm{~kb}$ fragment of the genome there are several open reading frames coding for a multitude of functional virus proteins namely; structural genes (gag, pol, and env), 
regulatory genes (tat, and rev), and accessory genes (vif, vpr, nef, and vpu). The gag gene provides the structural elements of the virus. The p24 part of the gene makes up the viral capsid whereas the $p 6$ and $p 7$ parts provide the nucleocapsid and $\mathrm{p} 17$ provides a protective matrix. The pol gene is a common feature of all retroviruses. It encodes for the reverse transcriptase enzyme that is responsible for the transcription of viral RNA into double-stranded DNA [Levy, 2007].

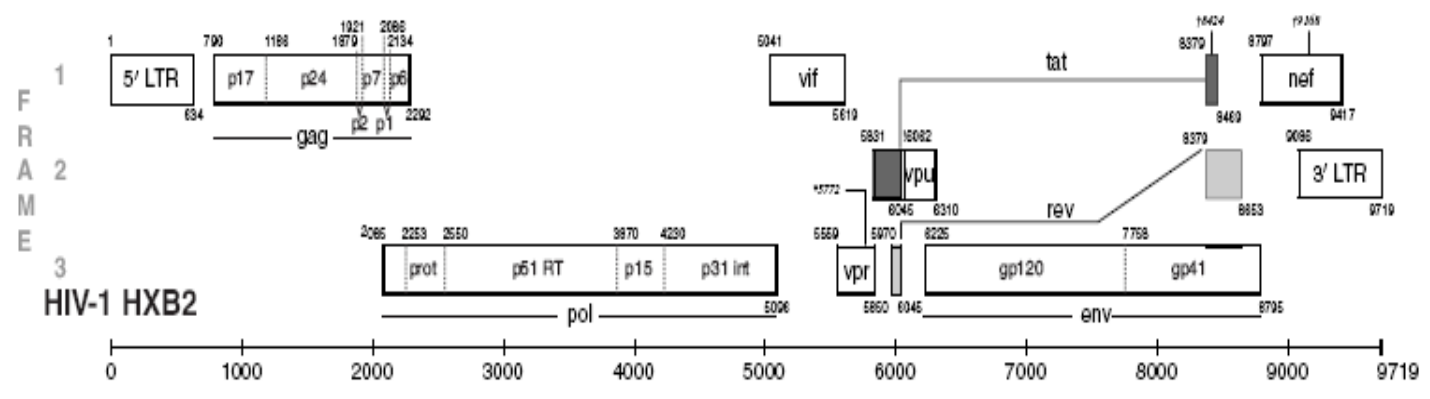

Figure 1.3: A diagrammatical representation of the genome layout of HIV-1. All three reading frames with all the most important genes are shown. All start and stop coordinates of genes on the diagram corresponds to that of the HXB2 reference strain. Exons 1 and 2 of tat and rev are indicated on the diagram with dark and light gray respectively [Adapted from http://www.hiv.lanl.gov/].

The pol gene also codes for the integrase and protease enzymes. Integrase is responsible for the integration of the double-stranded DNA into the host cells genome [Dicker et al, 2007]. The gag and pol genes do not produce their proteins in their final form, but as a large polypeptide. HIV protease then cleaves these large protein segments into separate functional units. The env gene encodes for a precursor protein of gp120 and gp41 called gp160, which is cleaved into the two functional proteins by the host cell's own enzymes. Env gp120 is exposed on the surface of the viral envelope and binds the virus to the CD4 receptors on the surface of any target cells. The glycoprotein gp41 is non-covalently bound to gp120, and facilitates the second step of viral entering into the target cells. The gp41 is originally found inside the viral envelope, but when gp120 binds to the CD4 receptor, gp120 undergoes a conformational change causing gp41 to become exposed on the viral envelope, where it can assist in the fusion of the virus with the host cell [Chan 
et al, 1997]. A summary of all the HIV genes and proteins are listed in Table 1.1 .

Table 1.1: The HIV-1 genes and proteins with their functions. [Adapted from Levy, 2007]

\begin{tabular}{|c|c|c|c|}
\hline \multicolumn{4}{|c|}{ HIV proteins and their function } \\
\hline Proteins & $\begin{array}{l}\text { Size } \\
\text { (kDa) }\end{array}$ & Function & Abbreviation \\
\hline \multirow{4}{*}{ Gag } & p24 & Capsid (CA), structural protein & $\mathrm{CA}$ \\
\hline & $\mathrm{p} 17$ & Matrix (MA) protein, myristoylated & MA \\
\hline & p7 & Nucleocapsid (NC) protein, helps in reverse transcription & NC \\
\hline & p6 & Role in viral budding ( $L$ domain) & - \\
\hline Polymerase & p66, p51 & Reverse Transcription (RT): RNase $\mathrm{H}$ - inside core & RT \\
\hline Protease & p10 & Gag/Pol cleavage and maturation & PR \\
\hline Integrase & p32 & Viral cDNA integration & IN \\
\hline \multirow{2}{*}{ Env } & gp120 & Envelope surface protein & SU \\
\hline & gp41 & Envelope transmembrane protein & TM \\
\hline Tat & $\mathrm{p} 14$ & Transactivation & Tat \\
\hline Rev & p19 & Regulation of viral mRNA expression & Rev \\
\hline Nef & $\mathrm{p} 27$ & Pleiotropic, can increase or decrease virus replication & Nef \\
\hline Vif & $\mathrm{p} 23$ & Promotes virion maturation and infectivity & Vif \\
\hline Vpr & p15 & Helps in virus replication, transactivation & Vpr \\
\hline Vpu & $\mathrm{p} 16$ & Helps in virus release, disrupts gp160:CD4 complexes & Vpu \\
\hline Vpx & p15 & Helps in entry and infectivity (Only in HIV-2 and SIV) & Vpx \\
\hline
\end{tabular}

Key: p (protein), gp (glycoprotein), - (no abbreviation), cDNA (complimentary DNA), RT (Reverse Transcriptase), mRNA (messenger RNA), and CD4 (cluster of differentiation 4)

Unlike certain oncogenic retroviruses, such as HTLV-I and -II in the retrovirus family, HIV-1 has no onc gene, but possess other unique genes such as; rev (facilitates the exportation of mRNA from the nucleus to the cytoplasm), tat (transactivation of HIV gene expression), vif (inhibits the cellular protein, APOBEC3G, from entering the virion at the time of budding), $v p r$ (plays an important role in regulating nuclear importation of the HIV-1 pre-integrated complex and is required for virus replication in non-dividing cells), vpu (facilitates viral budding), and nef (ensuring $\mathrm{T}$ cell activation and the establishment of a persistent state of infection) [Levy, 2007]. 


\subsubsection{The life cycle of HIV-1 (Replication)}

The human immunodeficiency virus (HIV) can infect a variety of immune cells such as CD4+ T-cells, Cytotoxic T-lymphocytes (CTLs), CD4+ monocytes, macrophages and CD4+ dendritic cells of the host's immune system [Chan et al, 1998]. The virus life cycle can be divided into two distinctive stages: the early establishment of infection and the later viral replication stage (Figure 1.4).

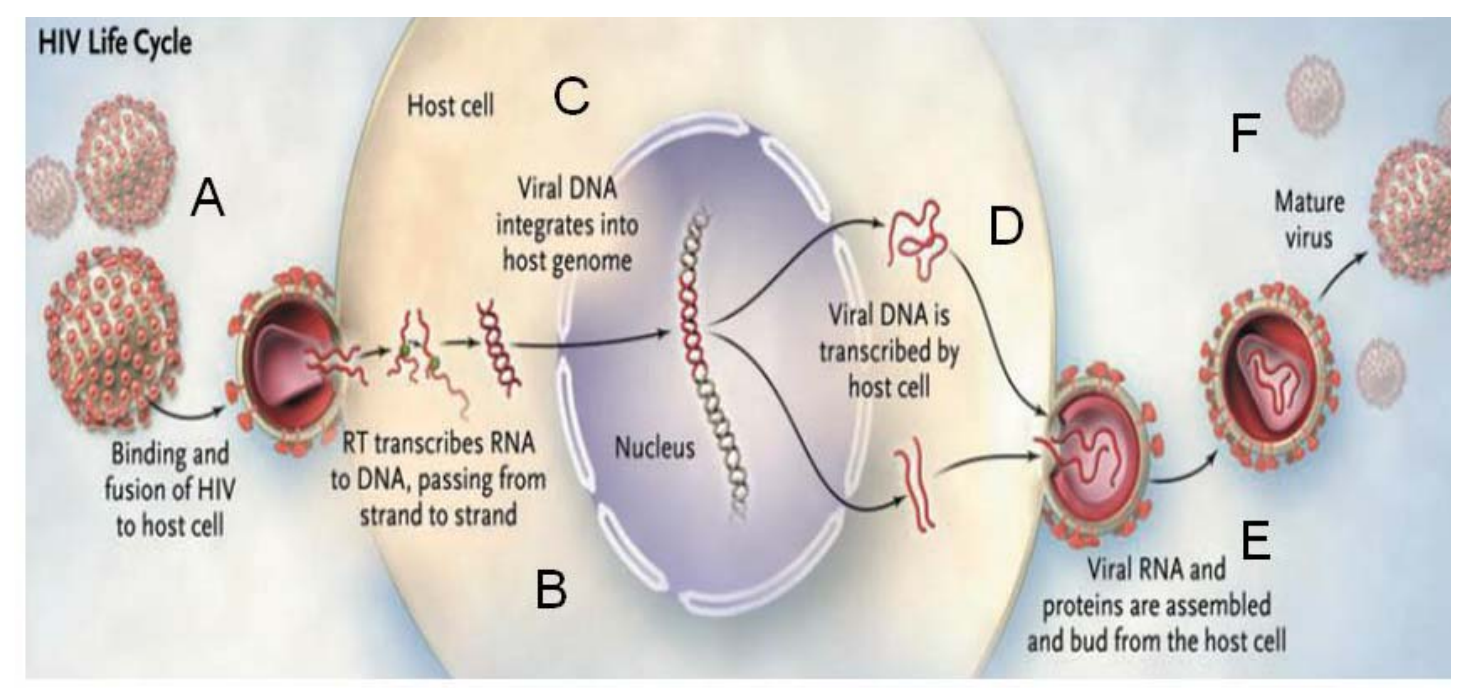

Figure 1.4: The HIV-1 life cycle. Stages $A-C$ represents the viral infection and establishment of the virus in the host cell and stages D - F the viral replication [Adapted from Taylor et al, 2008].

Successful infection of a target cell is accomplished through a series of virushost cell interactions. These include the binding of the virus to the cell surface, the fusion of the virus and the cell membrane, entry of the virus capsid into the cytoplasm of the host cell, the reverse transcription of RNA to DNA and the incorporation of the viral DNA into the nucleus of the host cell [Chan et al, 1998]. Briefly, entry to the cell begins through interaction of the trimeric envelope complex (gp160 spike) and both CD4 and a chemokine receptor (generally either CCR5 or CXCR4) on the cell surface [Levy, 2007]. The first step in fusion involves the high-affinity attachment of the CD4 binding domains of gp120 to CD4. Once gp120 is bound with the CD4 protein, the envelope complex undergoes a structural change, exposing the chemokine binding domains of gp120 and allowing them to interact with the target 
chemokine receptor. This allows for a more stable two-pronged attachment, which allows the $\mathrm{N}$-terminal fusion peptide gp41 to penetrate the cell membrane [Wyatt and Sodroski, 1998].

After successful fusion p24 is released into the cytoplasm, which uncoats to release the viral RNA into the host cells cytoplasm. The RNA is then reverse transcribed using the host cells reverse transcriptase enzyme to synthesis a double-stranded DNA copy of its RNA. With the help of the viral integrase the newly synthesized double-stranded copy of DNA is then incorporated into the host cells genome.

Once integration has occurred in a host cell the cells progeny will also be infected [Levy, 2007]. The provirus establishes latency in the infected cell. New virions are then synthesized from the integrated provirus. When a host cell is infected by two or more viruses there is a possibility that two different genomes may be transcribed into the same virion. The successful infection, integration and translation, of these virions into new host cells will then lead to the production of proviral genomes that are recombinants of the two viral genomes [Rodrigo and Learn, 2000]. Viral recombination in HIV-1 is made possible by the diploid (two RNA molecules) nature of the viral genome. Viral recombination of HIV-1 occurs during the reverse-transcription step, before viral integration and dependence on the co-packaging of two different viral genomes. This requires simultaneous infection of the same cell by two different strains and subsequent integration of two different parentalgeneration proviruses in the same nucleus (Figure1.5) [Taylor et al, 2008].

The later viral replication stage starts as soon as the viral DNA is transcribed into RNA by the host cells DNA polymerase. Spliced and unspliced viral mRNA is then exported to the cytoplasm of the host cell for translation and virus formation. Spliced viral mRNA is translated into viral proteins in the cytoplasm of the host cell. The virus capsid incorporates an unspliced copy of viral mRNA into a newly formed particle on the inner surface of the host membrane [Gelderblom, 1997]. New virions are produced as the virus buds through a region of the host's cell membrane. As the virion buds through the host cells membrane, outer surface proteins of the host cell become 
associated with the virus. The host cell dies from the effect of continuous immune activation that occurs in HIV-1 infected patients [Goldberg and Stricker, 1999]. Today it is widely hypothesized that the apoptosis of immune cells in infected patients is the major cause of the severe depletion of CD4+ Tcells and the eventual paralysis of an individual's immune system.

The intense study of the viral life cycle of HIV has been a key to developing valuable antiretroviral drugs against HIV-1. Today a wide range of antiretroviral drugs can be used to target up to six different stages of the viral life cycle including viral entry, reverse transcription, integration, expression of viral proteins, viral assembly and viral release [van Rossum AMC et al, 2002].

\subsection{Diversity of HIV-1}

HIV-1 is characterized by a high degree of genetic variation driven by a wide range of factors, such as the lack of a proofreading ability by its relatively weak reverse transcriptase [Op de Coul et al, 1997; Roberts et al, 1988], the rapid turnover time of HIV-1 in vivo [Ho et al, 1995], host selective pressures [Michael, 1999], and recombination events in dually infected patients [Temin, 1993]. The rate of sequence variation across the genome of HIV varies, with the highest degree of sequence variation in the env gene, intermediate amounts in the gag and a low degree in the pol gene [Shankarappa et al, 1999]. Thus, no two strains are identical and even in infected individuals; HIV is present in a swarm of micro variants (quasispecies) that are highly related, but genetically distinct.

The family of human immunodeficiency viruses consists of groups of genetically distinct retroviruses. The most basic division of the viruses that cause AIDS in humans is the human immunodeficiency virus type 1 (HIV-1) and type 2 (HIV-2). HIV-1 can be divided into three subgroups; Group M, N and $O$ and each of them have arisen due to a single zoonotic transmission from non-human primates. Group $M$ in itself is made up of nine subtypes, several sub-subtypes and 43 circulating recombinant forms (CRF's) [De Leys et al, 1990; Gurtler et al, 1994]. New data also support the idea that a subtype pattern can be found within group O sequences [Lemey et al, 2004]. 
The subtype classification that is used for HIV is based on a phylogenetic system, which means that subtypes are grouped on their inferred evolutionary relationship, rather than on other characteristics such as serological reactivity, phenotype, co-receptor usage and many other possible biological characteristics. This sets HIV viral subtype classification apart from other older viral pathogens where serological subtyping is the norm. HIV was discovered during the times in which PCR and sequencing technologies were discovered, which lead to the use of such techniques for the subtype classification instead of the older method of serology which was widely used for other older viral pathogens [Rodrigo and Learn, 2000]. The HIV-1 classification system was first used in 1992. By the end of 1992, five subtypes were known for env ( $A$ through E) and four for gag (A through D) [Myers et al, 1992; Louwagie et al, 1993]. Since then the classification system has been continually updated as new viral isolates were sequenced and new data became available. In 1993, subtypes $F, G$ and $H$ were added. Since then there has been many additions to the classification system [http://www.hiv.lanl.gov./].

Due to all these changes in the field of subtype classification, new criteria for assigning a sequence to an existing subtype and for creating new subtypes or sub-subtypes were decided on in September of 1999. These criteria set out to give more order to the classification system and set out strict rules for the creation of new groups, subtypes and sub-subtypes [Robertson et al, 2000]. The classification scheme of HIV-1 into subtypes has proven useful in the phylogenetic analysis for clarifying epidemiological relationships and possible ancestry of HIV and the classification of new sequences. It has given us a clear picture of the worldwide spread of the epidemic and provided us with information on how HIV has entered different countries, where patterns differ depending on epidemiological factors. In some cases there have been isolates that did not fit the subtype classification system very well. Some were found to be part of newly discovered subtypes, such as the subtype $F$ which was then sub-divided into sub-subtypes [Potts et al, 1993]. Others were later found to be recombinants that could not be assigned to a subtype unambiguously, but were more likely a mix of a wide variety of subtypes [Carr et al, 1996; Gao et al, 1996]. 
Advances in full-genome amplification and sequencing of HIV made the identification of circulating and unique recombinant forms (CRFs and URFs respectively) much easier. These isolates are the result of recombination between subtypes within a dually infected person (Figure 1.5), from which the recombinant forms are then passed on to other people. The progeny of such a recombinant virus are classified as a CRF if they have been identified in three or more epidemiologically unlinked people. If a particular recombinant form has only been identified in one or two cases and thus are of little epidemiological value then such viruses are classified as unique recombinant forms [Rodrigo and Learn, 2000].

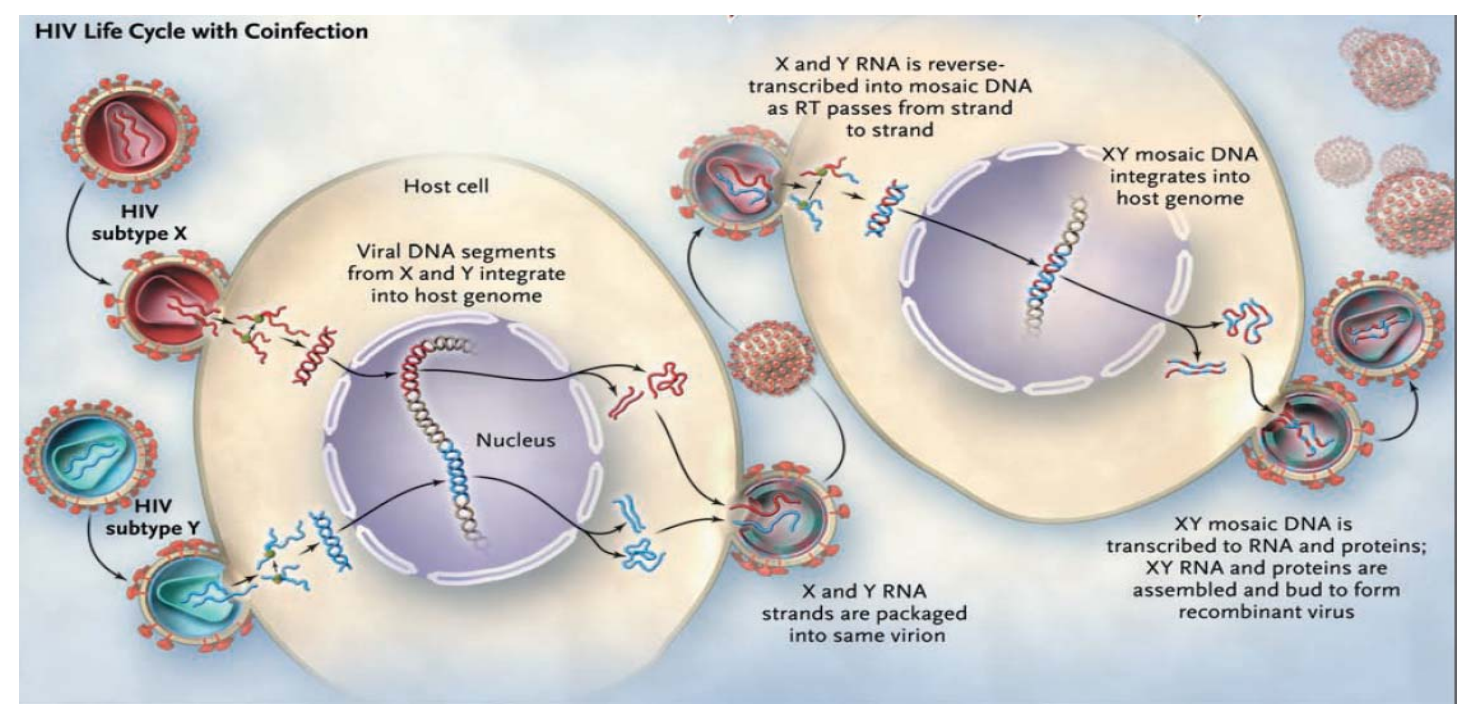

Figure 1.5: Creation of a URF in a coinfected individual. The individual is coinfected with two different subtypes $X$ and $Y$, where the genome of the URF represents part of both parental strands [Taylor et al, 2008].

\subsubsection{Global diversity of HIV-1}

Globally group $M$ is the predominant circulating HIV-1 group. Group M HIV-1 can be divided into 9 subtypes (A, B, C, D, F, G, H, J and K), sub-subtypes (A1-A3 and F1-F2), circulating recombinant forms (a total of 43 have been identified to date) and several unique recombinant forms [Gurtler et al, 1994; Janssens et al, 1994; Taylor et al, 2008]. In some cases, subtypes can be linked to a specific geographical region or epidemiological group [Hemelaar et al, 2006]. These distribution patterns are either the consequence of accidental trafficking (due to international travel) or due to a prevalent route of 
transmission, which results in a strong advantage for a specific subtype to become dominant within a specific region or country [Myers, 1994]. Recent academic data indicates that the most prevalent HIV-1 genetic forms are subtypes A, B, and C, with subtype C accounting for as much as $50 \%$ of all infections worldwide [Ariën et al, 2007].

Molecular epidemiology studies have shown that, with the exception of subSaharan Africa where most subtypes and circulating recombinant forms can be found, there is a specific geographic distribution pattern for HIV-1 subtypes (Figure 1.6).

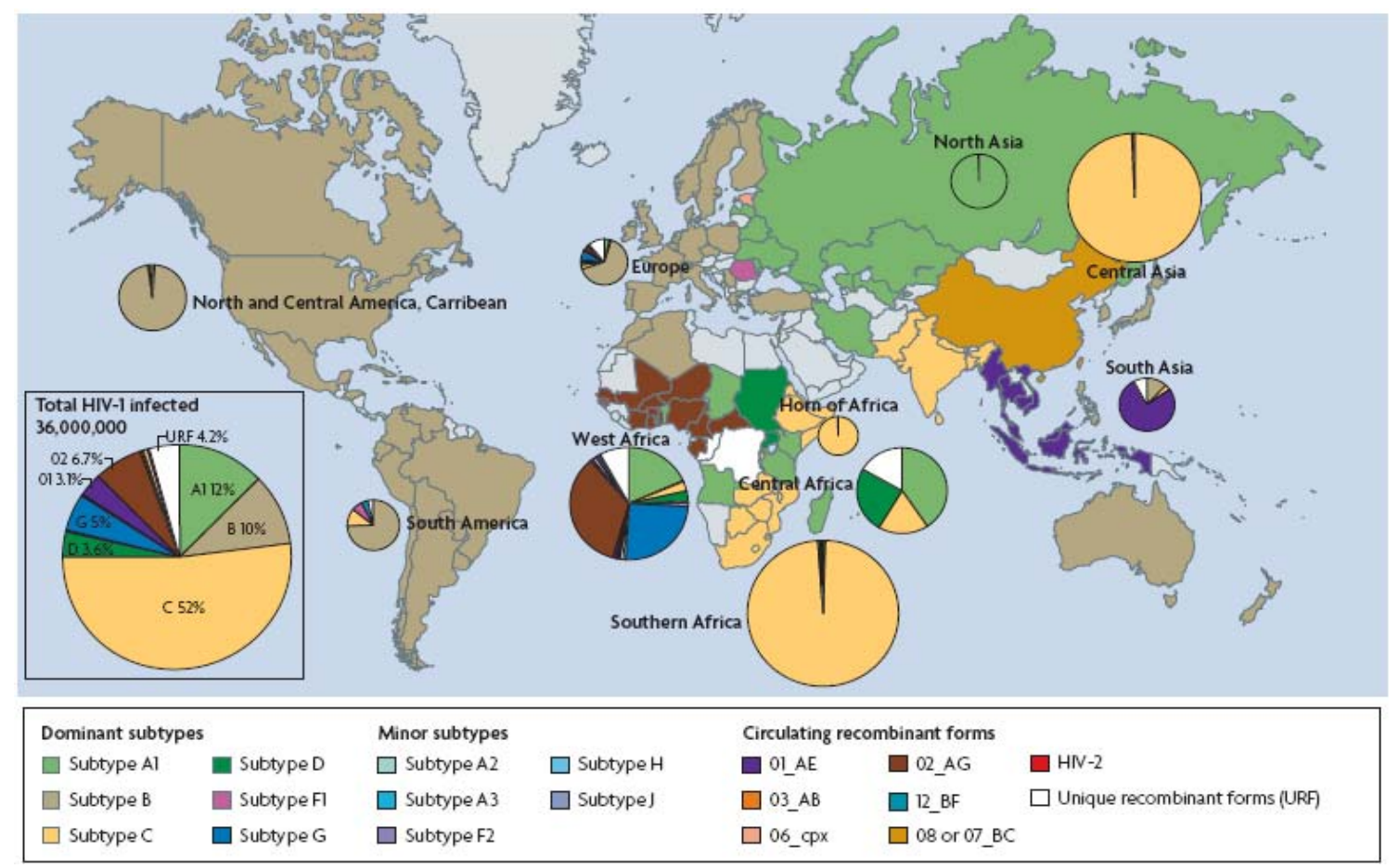

Figure 1.6: Global distribution of different HIV-1 subtypes and circulating recombinant forms. [Adapted from Ariën et al, 2007]

Subtype A viruses are most prevalent in areas of central and eastern Africa (Kenya \& Tanzania) and in the East European countries formerly part of the Soviet Union [Buonaguro et al, 2007]. These areas where subtype A viruses co-circulate with other viral subtypes have also seen the rise of recombinant viruses. Some areas have seen the rise of $A$ recombinant viral forms due to the co-circulation of other viral subtypes in the same geographical area e.g. 
the prevalence of subtypes $A$ and $B$ which lead to the rise of $A B$ recombinant viruses in Eastern Europe [Liitsola et al, 1998] and the co-circulating of subtype $A$ and $D$ viruses in East African countries such as Kenya which lead to the rise of $A D$ recombinants [Songok et al, 2004; Dowling et al, 2002].

Subtype B is the major genetic clade in the rest of Europe, the Americas, Japan and Australia, but is also found in large numbers in the countries of Southeast Asia, Northern Africa and the Middle East and amongst homosexual men from South Africa and Russia. Subtype C HIV-1 is predominant in the countries of Southern Africa and the Indian subcontinent [Buonaguro et al, 2007].

Of the 43 CRF's known to us, some 20 reports have been made of CRF's been isolated in areas where the parental strains of the recombinant viral forms are cocirculating. The existence of multiple subtypes and CRF's within the same region increases the probability that individuals will become infected with different HIV-1 genetic forms, which can exchange parts of their genetic material, and result in the formation of recombinant viruses [McCutchan, 2006]. The role that circulating recombinant forms or CRF's are playing in the global HIV-1 pandemic is increasingly being recognized, with CRF's accounting for more than $18 \%$ of global infections [Osmanov et al, 2002; Hemelaar et al, 2006; Peeters, 2000]. In some areas of the world, CRF's represent the dominant form of HIV-1 such as in Southeast Asia (CRF01_AE) [Menu et al, 1996] and in West and West Central Africa (CRF02_AG) [McCutchan et al, 1999; Njai et al, 2006].

CRF01_AE (Figure 1.7) plays a major role in the HIV-1 epidemic in Southeast Asia. This subtype is responsible for more than $75 \%$ of the infections within the region and accounts for an estimated $4.7 \%$ worldwide infections [Hemelaar et al, 2006]. This subtype was first identified in Thailand in the late 1980's [McCutchan et al, 1992; Carr et al, 1996]. It was first classified as a new clade called subtype "E" but after full-length sequence analysis of these isolates it became apparent that the virus appeared to have a mosaic-like structure, with the gag gene clustering with other subtype $A$ isolates and the 
env genes from clade E [Leitner et al, 2005; Carr et al, 1996; Gao et al, 1996]. To date the parental clade $E$ strain has not been found. Extensive studies of CRF01_AE have shown that the isolate was introduced into Thailand from Africa and from there the isolate has spread to other counties in Southeast Asia where it has become the most prevalent form of HIV-I where it is linked to heterosexual sex workers and intravenous drug users within the region [Anderson et al, 2000].

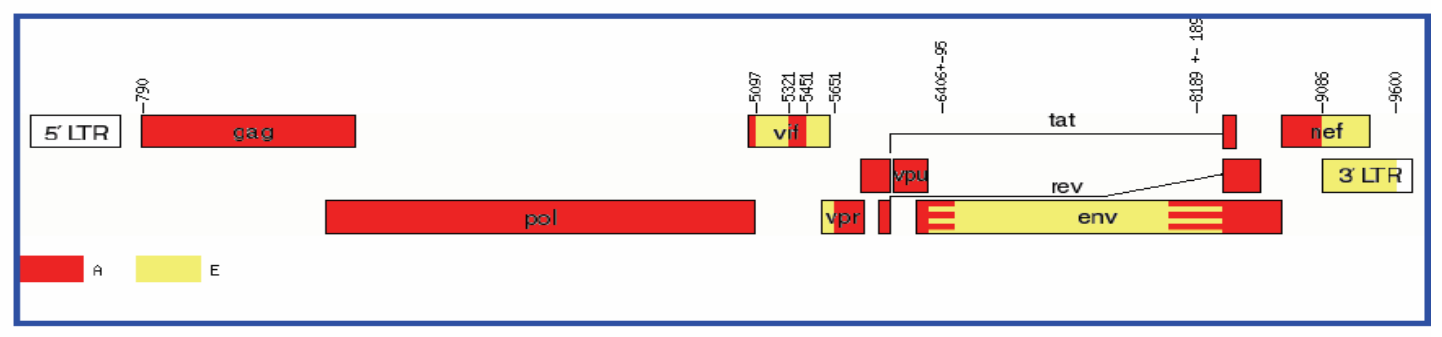

Figure 1.7: The genomic structure of a CRF01_AE isolate. The yellow areas correspond with HIV-I subtype E and the red areas with HIV-1 subtype A. The breakpoints of the recombination events are also shown (breakpoint coordinates relative to the reference strain HXB2) [Adapted from LANL database, http://www.hiv.lanl.gov/].

\subsubsection{HIV-1 diversity in Africa}

The African continent is home to the largest portion of all HIV infections in the world with as many as 23.6 million people living with HIVIAIDS [UNAIDS, 2008]. A multitude of subtypes and circulating recombinant forms can be found in Africa which is possibly due to the African origin of HIV-1 [Torques et al, 1999; Vidal et al, 2000]. The distribution and occurrence of different subtypes within African populations are not generally linked to a particular lifestyle habit as it would be in other parts of the world. In Central and Eastern Africa subtype A1 and D HIV-1 is the most prevalent form of HIV. Subtype C is dominating the epidemic in Southern Africa where it can account for as many as 95 percent of all HIV infections (Figure 1.8). Due to the importance of subtype C HIV within the Southern African region this subtype has been extensively studied in the past [Bell et al, 2007; Engelbrecht et al, 2001; Scriba et al, 2002; Bessong et al, 2005; Hunt et al, 2003; Papathanasopoulos et al, 2003b; zur Megede et al, 2002]. 


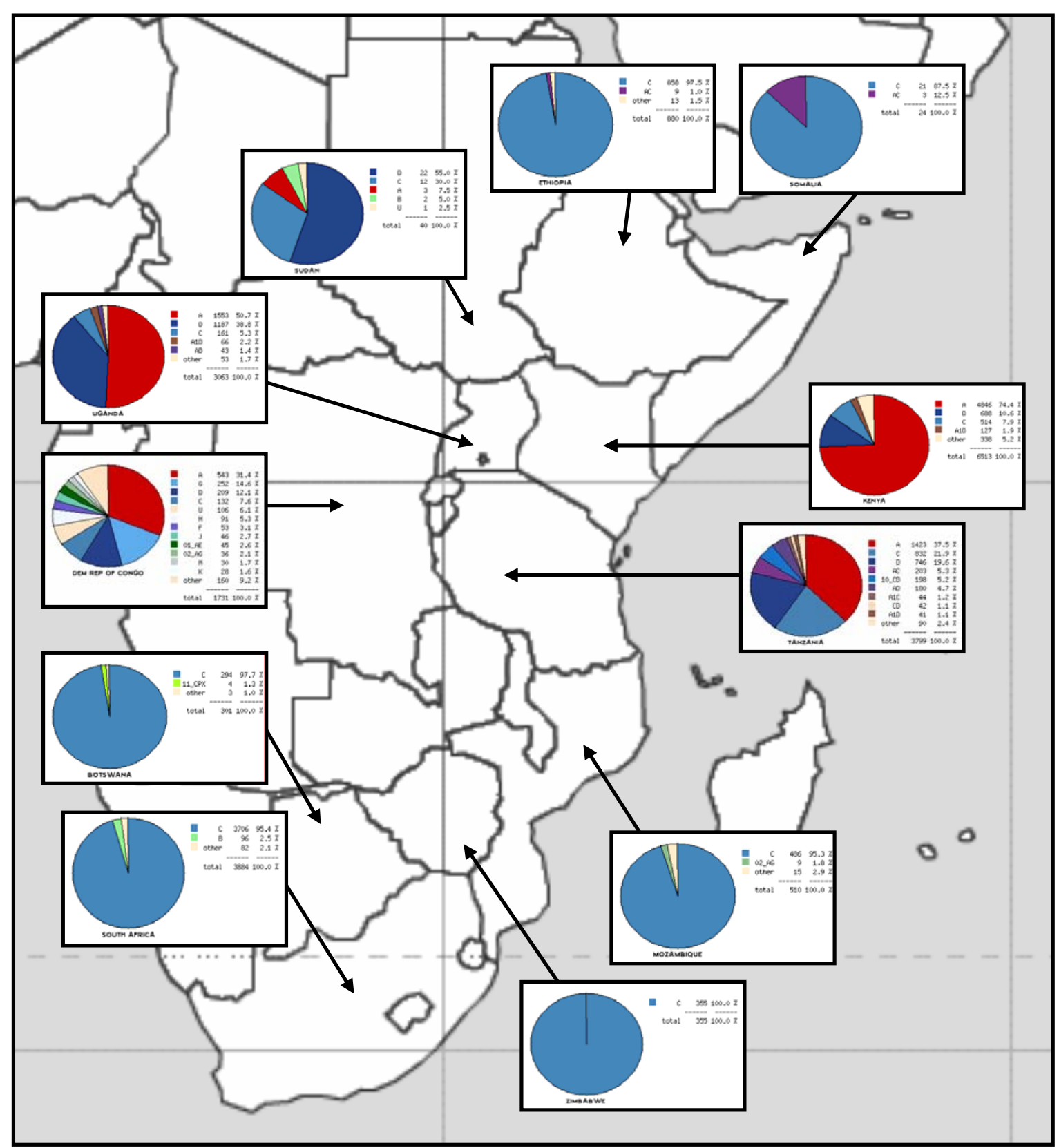

Figure 1.8: Subtype distribution of HIV-1 in a handful of sub-Sahara African countries [Adapted from: http:www.hiv.lanl.gov./].

CRF02_AG (Figure 1.9) which is the dominant form of HIV in Western and Central Africa also contributes considerably to the global epidemiology of the virus accounting for an estimated $4.6 \%$ of worldwide infections [Hemelaar et al, 2006].

Initially the CRF02_AG viral subtype was described as a divergent lineage within HIV-1 subtype A, based on partial gag and env sequences [Howard and 
Rasheed, 1996]. After full length sequences of these isolates were obtained, it was recognized as a complex mosaic virus of alternating subtype $A$ and subtype $G$ regions [Carr et al, 1998]. This viral form of HIV-1 have been isolated in various regions ranging from West Africa to East African countries and a CRF02_AG isolate have also been identified in South Africa. [Bredell et al, 2002, Jacobs et al, submitted] In countries of West and Central Africa, such as Nigeria and Cameroon, this recombinant form is responsible for between $50-70 \%$ of new infections [Andersson et al, 1999; McCutchan et al, 1999; Fischetti et al, 2004].

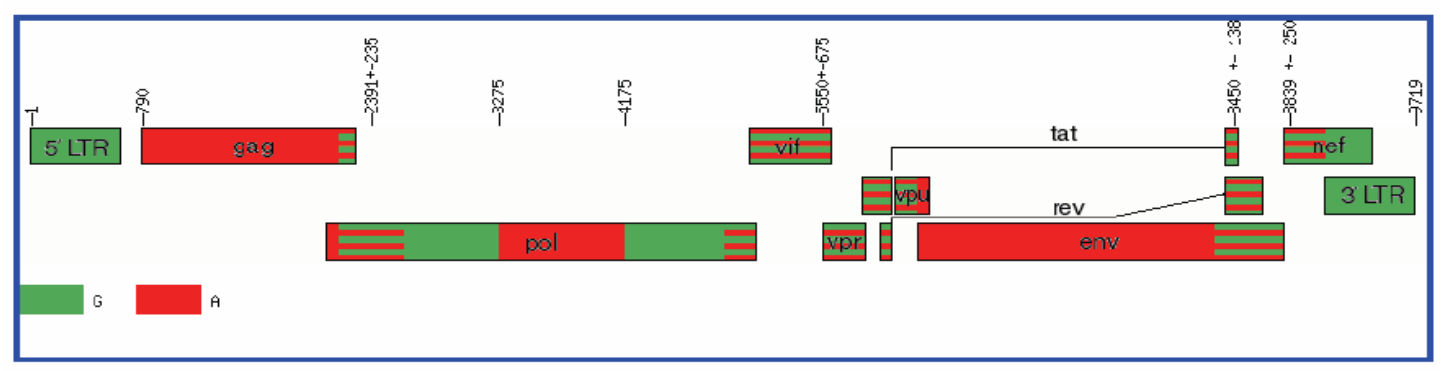

Figure 1.9: The genomic structure of CRF02_AG. The green areas correspond with HIV-I subtype $G$ and the red areas with HIV-1 subtype A. The breakpoints of the recombination events are also shown (breakpoint coordinates relative to the reference strain HXB2) [Adapted from LANL database, http://www.hiv.lanl.gov/].

\subsubsection{HIV-1 diversity in South Africa}

South Africa is currently in the grip of one of the most devastating AIDS epidemics in the world. By the end of 2007, 6.2 million people were living with HIV in South Africa, with countless more being affected by the lost of family and loved ones. UNAIDS estimated that there were 1.4 million South African children orphaned by AIDS in 2007 [UNAIDS, 2008]. The UNAIDS report of 2008 estimate that almost 1,000 AIDS related deaths occurring every day within the country [UNAIDS, 2008].

In 1982 the first reported case of AIDS in South Africa was documented, with the first virus isolation done in 1984 [Ras et al, 1983; Becker et al, 1985]. Initially HIV infections seemed to be occurring mainly amongst homosexual white men [Sher, 1989]. By the start of the 1990's it became apparent that a 
second HIV pandemic, amongst the heterosexual indigenous black population, was occurring within the country [Williamson et al, 1995]. By 1990 an estimated 74,000 people were living with HIV in South Africa [Department of Health, 2005]. The following year the number of diagnosed heterosexuallytransmitted HIV infections equaled the number of infections transmitted through men having sex with other men. Since then heterosexual transmission has dominated the epidemic in the country. The most rapid increase in South Africa's HIV prevalence took place between 1993 and 2000, during which time the country was distracted by major political changes. While the attention of the South African people and the world's media was focused on the political and social changes occurring in the country, HIV was rapidly becoming more widespread. Subtype C HIV-1 is responsible for nearly 95 percent of all infections within the country.

Over the past years subtype C HIV-1 has been extensively studied in South Africa due to its immense importance [Jacobs et al, 2006; Hunt et al, 2003; Papathanasopoulos et al, 2002; Rousseau et al, 2006; de Oliveira et al, 2003; Gordon et al, 2003; zur Megede et al, 2002]. To date only a few papers have been published on complete genomes of non-subtype C HIV-1 isolates in South Africa [Papathanasopoulos et al, 2001; Loxton et al, 2005]. A lot of research has been conducted on HIV-1 over the years within the country. To date several papers on a wide range of HIV-1 subtypes have been published on subgenomic fragments (Table 1.2) or near full-length sequences (Table 1.3). These viruses were isolated from several geographical locations within the country. The majority of HIV research has been conducted within the three biggest urban areas, Johannesburg, Cape Town, and Durban, but smaller studies has also been conducted in other areas of the country, such as Limpopo and Mpumalanga. 
Table 1.2: Summary of published data of subgenomic fragments of HIV-1 isolates from South Africa.

\begin{tabular}{|c|c|c|c|c|}
\hline \multicolumn{5}{|c|}{ Characterization of subgenomic regions } \\
\hline Publication & $\begin{array}{l}\text { Number of } \\
\text { samples }\end{array}$ & Method of Subtyping & Region of Genome & Subtypes \\
\hline \multirow[t]{3}{*}{ Engelbrecht et al, 1994} & \multirow[t]{3}{*}{17 Samples } & \multirow{3}{*}{$\begin{array}{l}\text { Serology, Sequencing \& } \\
\text { Phylogenetics }\end{array}$} & \multirow[t]{3}{*}{ env gp41 } & Subtype B \\
\hline & & & & Subtype D \\
\hline & & & & Subtype C \\
\hline \multirow[t]{3}{*}{ Becker et al, 1995} & & \multirow[t]{3}{*}{ Sequencing and Phylogenetics } & \multirow[t]{3}{*}{ gag \& env } & Subtype B \\
\hline & & & & Subtype D \\
\hline & & & & Subtype C \\
\hline \multirow{4}{*}{$\begin{array}{l}\text { van Harmelen et al, } \\
1997\end{array}$} & \multirow[t]{4}{*}{61 Samples } & \multirow[t]{4}{*}{ HMA \& partial sequencing } & \multirow[t]{4}{*}{ V3 - V5 or gag } & Subtype B \\
\hline & & & & Subtype C \\
\hline & & & & Subtype D $(n=1)$ \\
\hline & & & & CRF01_AE $(n=1)$ \\
\hline Bredell et al, 1998 & 44 Samples & HMA \& sequencing & V3 - V5 env region & All subtype $\mathrm{C}$ \\
\hline \multirow[t]{2}{*}{ Engelbrecht et al, 1999} & \multirow[t]{2}{*}{81 Samples } & \multirow{2}{*}{$\begin{array}{l}\text { Serology (cPEIA) and } \\
\text { Phylogenetics }\end{array}$} & \multirow[t]{2}{*}{ V3 region of env } & Subtype C \\
\hline & & & & Subtype B \\
\hline \multirow{4}{*}{$\begin{array}{l}\text { van Harmelen et al, } \\
1999\end{array}$} & \multirow[t]{4}{*}{87 Samples } & \multirow[t]{4}{*}{ RFLP } & \multirow[t]{4}{*}{ gag } & Subtype A $(n=2)$ \\
\hline & & & & Subtype B $(n=28)$ \\
\hline & & & & Subtype C ( $n=56)$ \\
\hline & & & & Subtype D $(n=1)$ \\
\hline \multirow[t]{4}{*}{ Hunt et al, 2001} & \multirow[t]{4}{*}{60 Samples } & \multirow[t]{4}{*}{ HMA \& Phylogenetics } & \multirow[t]{4}{*}{ gag \& env } & Subtype C $(n=43)$ \\
\hline & & & & Subtype A $(n=2)$ \\
\hline & & & & Subtype B $(n=3)$ \\
\hline & & & & Several Recombinants \\
\hline Engelbrecht et al, 2001 & 13 Samples & Phylogenetics & gag \& env & All subtype C \\
\hline \multirow[t]{2}{*}{ Bredell et al, 2002} & \multirow[t]{2}{*}{10 Samples } & \multirow[t]{2}{*}{ HMA \& partial sequencing } & \multirow[t]{2}{*}{ gag \& env } & Subtype $A(n=2)$ \\
\hline & & & & C CA, CD, G, AG and D \\
\hline
\end{tabular}




\begin{tabular}{|c|c|c|c|c|}
\hline \multicolumn{5}{|c|}{ Characterization of subgenomic regions - Continued } \\
\hline Publication & $\begin{array}{l}\text { Number of } \\
\text { samples }\end{array}$ & Method of Subtyping & Region of Genome & Subtypes \\
\hline Scriba et al, 2002 & 14 Samples & Phylogenetics & 5' LTR, nef, tat and rev & All subtype $\mathrm{C}$ \\
\hline \multirow[t]{2}{*}{ Gordon et al, 2003} & \multirow{2}{*}{72 Samples } & \multirow[t]{2}{*}{ Phylogenetics } & \multirow[t]{2}{*}{ pol and env C2V5 } & Subtype $C(n=71)$ \\
\hline & & & & CD Recombinants $(n=1)$ \\
\hline Bessong et al, 2005 & 42 Samples & HMA \& sequencing & gag \& env & All Subtype C \\
\hline Bell et al, 2007 & 20 Samples & Phylogenetics & vif, vpr \& vpu & All subtype C \\
\hline \multirow[t]{2}{*}{ Jacobs et al, 2008a } & \multirow[t]{2}{*}{50 Samples } & \multirow[t]{2}{*}{ Phylogenetics } & \multirow{2}{*}{ vif } & Subtype C $(n=48)$ \\
\hline & & & & Subtype B $(n=2)$ \\
\hline \multirow[t]{4}{*}{ Jacobs et al, 2008b } & \multirow{4}{*}{$\begin{array}{l}140 \\
\text { Samples }\end{array}$} & \multirow[t]{4}{*}{ Phylogenetics } & \multirow[t]{4}{*}{ pol sequences } & Subtype $C(n=133)$ \\
\hline & & & & Subtype B $(n=5)$ \\
\hline & & & & CRF02_AG $(n=1)$ \\
\hline & & & & Subtype G $(n=1)$ \\
\hline \multirow[t]{5}{*}{ Jacobs et al, submitted } & \multirow{5}{*}{$\begin{array}{l}410 \\
\text { Samples }\end{array}$} & \multirow{5}{*}{$\begin{array}{l}\text { Serology (cPEIA) and } \\
\text { Phylogenetics }\end{array}$} & \multirow[t]{5}{*}{ V3 region of env } & Subtype C $(n=341)$ \\
\hline & & & & Subtype B $(n=36)$ \\
\hline & & & & Subtype A $(n=7)$ \\
\hline & & & & Subtype D $(n=3)$ \\
\hline & & & & Several Recombinants \\
\hline
\end{tabular}

Key: n (number) 
Table 1.3: Published data of near-full length HIV-1 sequences from South Africa.

\begin{tabular}{|c|c|c|c|}
\hline \multicolumn{4}{|c|}{ Full length-genome characterization } \\
\hline Publication & $\begin{array}{l}\text { Number of } \\
\text { samples }\end{array}$ & Method of Subtyping & Subtypes \\
\hline van Harmelen et al, 2001 & 4 Samples & Sequencing and Phylogenetics & Subtype C ( $n=4)$ \\
\hline \multirow[t]{3}{*}{ Papathanasopoulos et al, 2002} & \multirow{3}{*}{4 Samples } & \multirow[t]{3}{*}{ Sequencing and Phylogenetics } & Subtype C ( $n=2)$ \\
\hline & & & A2C Recombinant $(n=1)$ \\
\hline & & & ACDGK Recombinant $(n=1)$ \\
\hline zur Megede et al, 2002 & 3 Samples & Sequencing and Phylogenetics & All subtype C \\
\hline Papathanasopoulos et al, 2003b & 2 Samples & Sequencing and Phylogenetics & All subtype $C$ \\
\hline Hunt et al, 2003 & 3 Samples & Sequencing and Phylogenetics & All subtype $C$ \\
\hline Loxton et al, 2005 & 4 Samples & Sequencing and Phylogenetics & All subtype D \\
\hline \multirow[t]{3}{*}{ Rousseau et al, 2006} & \multirow{3}{*}{244 Samples } & \multirow[t]{3}{*}{ Sequencing and Phylogenetics } & Subtype C $(n=241)$ \\
\hline & & & Subtype B $(n=1)$ \\
\hline & & & CA Recombinants $(n=2)$ \\
\hline Jacobs et al, 2007 & 1 Sample & Sequencing and Phylogenetics & Subtype D ( $(n=1)$ \\
\hline
\end{tabular}

Key: n (number) 


\subsection{Diversity within the HIV - 2 genome}

Since the discovery of HIV-2 in Western Africa in the mid-1980's a great deal has been learned about the epidemiology, spread and pathogenesis of the virus [Markovitz, 1993]. Unlike HIV-1, HIV-2 has not spread all over the world, but is mostly confined to areas of West Africa. Analyses of HIV-2 have revealed the existence of five major lineages within the cluster, which are termed HIV-2 groups A - E [Gao et al, 1994; Hasegawa et al, 1989].

\subsection{Methods used in the phylogenetic analysis of HIV}

Phylogenetics is the science of estimating the evolutionary relationship, which is based on the comparison of DNA or protein sequences with the phylogeny ultimately depicted in the form of an evolutionary tree [Graur and Li, 1999; Salemi and Vandamme, 2003]. The use of trees to depict such hypotheses goes back to the start of the field of evolution in Charles Darwin's days. It is only until recently that methods have been developed to numerically calculate trees through quantitative methods. In the modern age of rapid gene sequencing, digitization of sequences and creation of sequence databases, molecular phylogeny has become a powerful tool for making sense of these vast amounts of data.

Before one can start with phylogenetic analysis of data, one needs to generate DNA sequence data. These procedures include the designing and testing of primers, the amplification of target genes or genomes, and the sequencing of amplified products [McCormack and Clewley, 2002]. These processes include a wide range of procedures and technical expertise on its own. The following section will take a look at some of the methods commonly used in modern phylogenetic analysis.

\subsubsection{Searching for homologous sequences}

The ultimate goal of phylogenetic analysis is to compare the evolutionary relationship between new sequences and other known sequences. One of the first things to do before starting analysis of data is to obtain homologous 
sequences. Today most genetic data, either in nucleic acid or amino acid formats, is stored in online accessible sequence databases with the three largest of these being; EMBL (European Molecular Biology Laboratory), GenBank (at NCBI), and the DDBJ (DNA Data Bank of Japan) [Salemi and Vandamme, 2003]. The BLAST (Basic Local Alignment Search Tool) is the most widely used method in modern molecular evolutionary biology to search for homologous sequences and can be performed on most of the genetic databases [Altschul et al, 1990]. Some databases were developed for specific uses such as the LANL (Los Alamos National Laboratory, Los Alamos, New Mexico, USA) which only contains HIV and SIV related sequences [http://www.hiv.lanl.gov/].

The HIV sequence database at Los Alamos was created in 1986 under the guidance of the AIDS Program of the US National Institute of Allergy and Infectious Diseases (NIAID) in association with the US Department of Energy (DOE) [Rodrigo and Learn, 2000]. It is a specialized molecular-sequence database that provides a wide range of services, free of charge to the global HIV research community. The HIV database, unlike other more general genetic databases such as Genbank, contains only sequences that relate to primate lentiviruses and a few primate genes and proteins that interact with HIV. Any data in the database is shared with the general databases, so that all the data also appears in the GenBank, EMBL, and DDBJ databases [Learn et al, 1996]. The HIV database also contains useful tools for working with sequences. One can search the database of sequences in a number ways: by genome region, country of origin, subtype, viral phenotype, year of sampling, similarity to a given sequence (BLAST) and health of patients (with respect to ARV therapies, CD4 counts and several other factors) [Holmes, 2000]. The database also contains several pre-built sequence alignments of nucleotides or amino acids of partial fragments or complete genomes. Other tools include the jpHMM (for subtype and recombinant identification) [Zhang et al, 2006; Schultz et al, 2006], sequence locator (useful for assigning a DNA fragment to a particular region of the HIV genome relative to HXB2) [http://www.hiv.lanl.gov/], RIP or Recombinant Identification Program (for 
recombinant virus identification) [Siepel et al, 1995] and gene cutter [http://www.hiv.lanl.gov/].

\subsubsection{Aligning homologous sequences}

Before phylogenetic trees can be draw one must first construct a multiple alignment containing the sequences of interest with homologous sequences that were obtained from a specific sequence database [Hogeweg and Hesper, 1984]. Accurate alignment of sequences is extremely important in the analysis of datasets. Only homologous sequences can be aligned with one another. Homologous sequences are any two sequences that share a common recent ancestor. One should distinguish between sequence similarity and sequence homology. Any sequences have some measurable similarity, but homology implies that this similarity is the result of a share common recent ancestor [Abecasis et al, 2007].

The most common method of constructing multiple alignments is by progressive alignment such as Clustal $\mathrm{W}$ or Clustal $\mathrm{X}$ [Salemi and Vandamme, 2003]. When a multiple alignment can be used to construct a phylogenetic tree then the converse is also true. With progressive alignment methods sequences are aligned in pairs to create a distance matrix based on their alignment scores. These scores are then downweighted according to how closely related the sequences are. This distance matrix is used to construct a Neighbor Joining (NJ) guide tree. The guide tree is used to cluster the sequences during the stepwise alignment, with the isolates that were clustered closest together being aligned with one another first. For further alignment these two sequences are treated as one with other sequences being aligned to them one by one. Clustal $W$ [Thompson et al, 1994] and Clustal $X$ [Thompson et al, 1997] are the most widely used programs for carrying out multiple alignments. These two programs are identical in terms of alignment methods, but Clustal $\mathrm{W}$ offers a simple text-based interface whereas Clustal $X$ has a more graphical interface which might be more user friendly [Salemi and Vandamme, 2003]. Before proceeding with further analysis one should always manually check the alignment with a sequence 
editing program such as BioEdit [Hall, 2001]. Often one can easily improve the alignment and in some cases it might be required to delete blocks containing gaps [Abecasis et al, 2007].

\subsubsection{Choosing a model of evolution}

Genetic sequences are not very informative regarding their evolutionary history. When we compare homologous sites in sequences, we only observe that the sequences are the same or different [Page and Holmes, 1998]. Evolution is caused by mutations which spread through populations by genetic drift or natural selection. These mutations can be caused by a number of events such as nucleotide substitutions, insertions, deletions or recombination events [Graur and Li, 1999]. Phylogenetic analysis makes certain assumptions about the process and rate of DNA substitutions or amino acid replacements in the model of evolution they employ [Salemi and Vandamme, 2003]. Point mutations can either by be due to transitions, when a purine nucleic base $(A, G)$ replaces another purine base or a pyrimidine base $(C, T)$ replaces another pyrimidine base, or transversions, when a purine is replaced by a pyrimidine base or vice versa [Graur and Li, 1999].

To study the dynamics of these changes in the sequences, one needs to use mathematical models that take into account different rates of nucleotide substitution. To date, a vast array of these models has been developed over the years by scientists [Li, 1997; Graur and Li, 1999; Salemi and Vandamme, 2003]. The first of these models, the Jukes and Cantor method, was developed as far back as the late 1960's [Jukes and Cantor, 1969]. The three most commonly used methods for the analysis of HIV datasets are the Kimura two-parameter model [Kimura, 1980]; the Hasegawa, Kishino, Yano (HKY) method [Hasegawa et al, 1985] and the General time-reversible (GTR) model [Rodriguez et al, 1990; Yang et al, 1994].

In most cases, as for HIV, the number of transitions is often higher than the rate of transversions. In 1980, Kimura developed an algorithm for estimating the number of nucleotide substitutions per site, which took into account the higher probability of transitional change [Kimura, 1980]. The model assumes a 
total rate of nucleotide substitution of: $\alpha+2 \beta$, where rate of transitions per site is $\alpha$ and the rate of transversions is $\beta$. At any particular site the nucleotide base can undergo three possible changes, one being a transition and the other two being transversions [Li, 1997].

The Hasegawa, Kishino, Yano (HKY) model was first described in 1985 by Hasegawa and co-workers [Hasegawa et al, 1985]. As in the case of the Kimura 2-parameter model, the HKY-model also allows for a transition/transversion bias, but unlike the Kimura 2-parameter model that estimates equal base frequencies, the HKY-model allows base frequencies to vary. Theoretically in a given sequence each nucleotide base (Adenine, Cytosine, Guanine, or Thymine) has an equal probability (0.25) of appearing, however it often does not hold true. Some organisms have a higher composition of guanine and cytosine which makes their DNA much more thermodynamically stable due to the higher concentration of triple hydrogen bonds in the DNA molecules. Thus it is clear when working with sequences that the use of a model which allows for base frequencies to vary would be much more useful and accurate than other models that do not allow for this [Page and Holmes, 1998].

The GTR or general time-reversible model is the most general, unbiased, independent, finite-sites, time-reversible model possible, and was first described in 1986 by Simon Tavaré [Tavaré, 1986]. The probability matrix of the GTR-model has six parameters so that each possible substitution has its own probability [Page and Holmes, 1998]. Thus this model allows not only for different base frequencies, but also for different rates for all six substitutions. For a time-reversible model, there is no assumption that substitutions preferentially change in a certain direction over time.

\subsubsection{Drawing phylogenetic trees}

A phylogenetic tree, much like a real tree, is made up of branches and nodes. The branches are connected to the nodes and the nodes are the point of branch divergence. Nodes can also be internal or external. External nodes represent the sequences from which the tree was constructed or operational 
taxonomic units (OUT's) whereas the internal nodes represent a common ancestor between two or more taxa (Figure 1.10).

Phylogenetic trees are usually drawn so that the branch lengths correspond to the amount of evolution between the two nodes they connect and such trees are termed additive trees. That means the longer the branches, the more divergent the sequences are. At the base of a phylogenetic tree is normally a root, which represents the oldest common ancestor of all the sequences in the tree [Hall, 2004]. Trees are rooted by using outgroups or an external point of reference. An outgroup may be anything that is not a natural member of the sequences or group of interest [Baldauf 2003; Salemi and Vandamme 2003].

Two main methods of calculating phylogenetic trees exist today: the distancematrix method, also known as clustering or the algorithmic method (e.g. UPGMA, neighbor-joining, or Fitch - Margoliash) and the discrete data method which is also known as the tree searching method (e.g. parsimony, maximum likelihood, or the Bayesian method) [Baldauf 2003].

The distance method is extremely easy and fast to use, but does not involve an evolutionary model. The distance (or percentage sequence difference calculated by pairing up two sequences in a matrix), is calculated for all pairwise combinations of all OTU's and then the distances are assembled into a tree [Baldauf 2003]. Thus sequences with the closest distances are grouped close together on the representative tree. The UPGMA or Unweighted Pair Group Method with Armetric Means, searches for the smallest value in the pairwise distance matrix to construct a phylogenetic tree [Sneath and Sokal, 1973] The neighbor-joining method sequentially finds its closest neighbors based on the internal branch lengths of the tree [Saitou and Nei, 1987], and the Fitch-Margoliash method evaluates all possible trees to find the one with the shortest overall branch lengths [Fitch and Margoliash, 1967].

The discrete data method, such as the maximum parsimony, maximum likelihood and Bayesian methods, examines each column of the multiple alignments separately and then searches for the tree that best accommodates all of this information. Discrete data analyses are rich in information because it 
creates a hypothesis for every column in the alignment and one can thus trace the evolution at a specific site in a DNA molecule (e.g. regulatory regions) [Baldauf 2003].

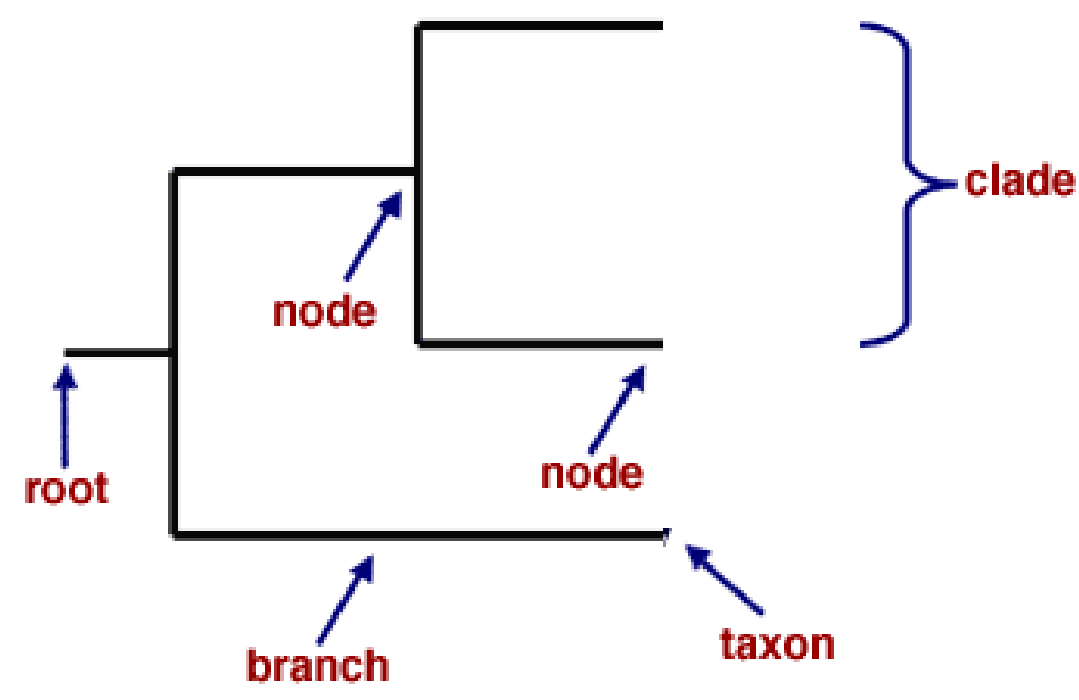

Figure 1.10: A simplistic representation of a phylogenetic tree. The tree indicates the root, branches, and nodes of the tree. A clade of are shown as well as a taxon (OTU) [Adapted from: http://www.talkorigins.org/].

The maximum parsimony and maximum likelihood methods use the theory of stepwise addition and branch swapping to search for the most representative phylogenetic tree. Through stepwise addition, branches are added in succession at different levels on the tree. Each level is then evaluated and the best tree is chosen before the addition of the next branch. Branch swapping techniques allow for the pre-defined rearrangement of the branches. The most common branch swapping techniques are tree bisection and reconstruction; nearest-neighbour interchange; and subtree pruning and regrafting [Salemi and Vandamme, 2003]. Maximum parsimony and maximum likelihood can both be performed with the PHYLIP software package [Felsenstein, 1982]. Baysian analysis is very much like that of maximum likelihood [Mau et al, 1999; Rannala and Yang, 1996]. Instead of seeking the tree that maximizes the likelihood of observing data, Bayesian analysis seeks those trees with the greatest likelihood of the given data. Baysian models search for the best tree 
which is consistent with both the evolutionary model of choice and the data in an alignment. Baysian analysis of datasets is the most commonly used method today, with new software, such as BEAST (Bayesian Evolutionary Analysis Sampling Trees) [Drummond and Rambaut, 2007].

\subsubsection{Detection of recombinant viruses}

Recombination within viral genomes, and especially within HIV's genome, is extremely common. Recombination within the HIV genome occurs when an individual is co-infected with multiple strains of the virus. To date, several methods have been developed for the identification of recombinant viruses. In some cases one can characterize several small subgenomic regions throughout the viral genome [Swanson et al, 2003]. This method, though widely used does have some drawbacks. One can miss small regions of recombination in-between the regions that were characterized. Recent advances in the field of viral DNA or RNA amplification (Figure 1.11), which makes the amplification of large fragments (6 - $36 \mathrm{kbp})$ possible, has made the identification of viral recombinants much easier [Salminen et al, 1995a; Nadai et al, 2008].

The importance of full genome characterization of samples has been described on several occasions in the past [Choi et al, 1997; Carr et al, 1996; Gao et al, 1996]. To successfully identify viral recombinants there must be enough genetic variation between the different lineages of the particular virus in order to confirm that genetic exchange has occurred. The basic strategy of recombination identification is to construct a multiple alignment containing the query sequence and several different isolates from the different lineages. When these alignments of full or near full-length genome sequences are analyzed with the use of software packages such as; Simplot (Similarity Plot) [Lole et al, 1999; Salminen et al, 1995b], RIP (Recombination Identification Program) [Siepel et al, 1995], or RDP3 (Recombination Detection Program) [Martin et al, 2004], one can see the full extent of viral recombination within a single isolate. 


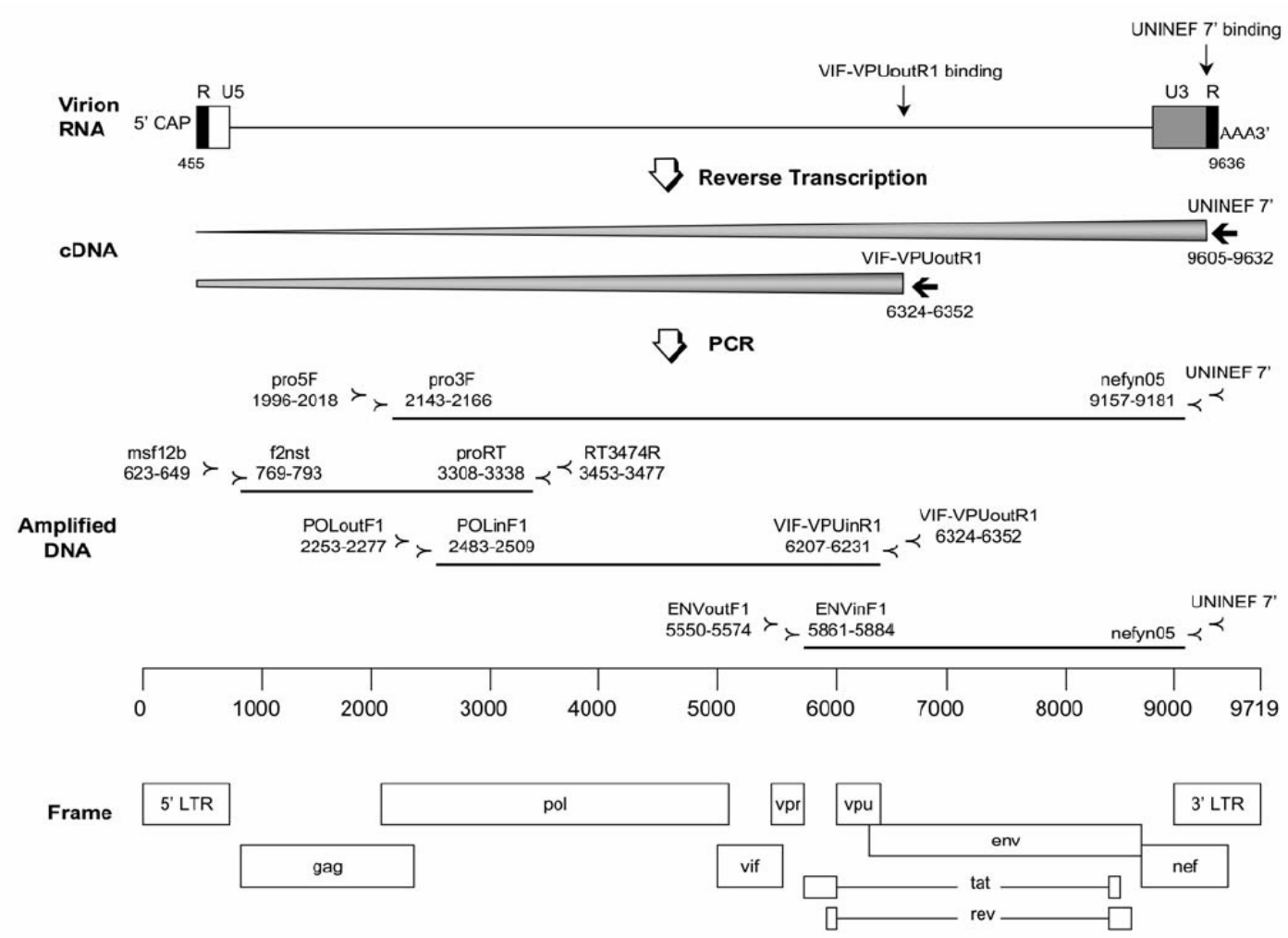

Figure 1.11: A graphical representation of a full-length amplification assay, for the characterization of a near full-length HIV-1 genome. Viral RNA is reverse transcribed into cDNA. PCR's are then performed in four overlapping fragments to amplify a near full-length genome of the virus. The methodology that was used here was developed as a standard protocol for the characterization of near full-length HIV-1 genomes from RNA [Nadai et al, 2008].

Simplot uses a sliding window approach moving across a multiple alignment in small increment steps to generate a similarity plot [Lole et al, 1999; Salminen et al, 1995b]. The program allows the user to query any sequence within the alignment and adjust the window and step sizes. The program is based on the Kimura 2-parameter substitution model. The recombination identification program (RIP) is one of the many online tools which are accessible from the LANL database [http://www.hiv.lanl.gov/; Siepel et al, 1995]. The program gives the user the opportunity to compare the query sequence against their own alignment or the pre-made alignment of the program itself. The pre-made alignment consists of several isolates from the most prominent viral subtypes (A1, B, C, D, F1, F2, G, H and CRF01_AE). Other subtypes such as $\mathrm{A} 2, \mathrm{~J}$ and $\mathrm{K}$ are only represented by a single sequence in the alignment. The large size of the pre-made alignment makes 
the identification of recombination events much easier. The program also allows the user to define the appropriate window size. It further allows for the simplifying of the results, with the use of the rerun application, and exports the graphs in a convenient manner [http://www.hiv.lanl.gov/].

\section{AIM OF THE STUDY}

The large variety of subtypes, sub-subtypes and circulating recombinant forms of HIV-1 complicates the development of effective vaccination strategies and has major implications for diagnostic assays and the effective treatment of infected people with anti-retroviral drugs. It is thus of the utmost importance that we continue monitoring the epidemiology of the HIV-1 epidemic. To date very few papers have been published on near full-length non-subtype C HIV type 1 viruses in South Africa [Papathanasopoulos et al, 2001; Loxton et al, 2005]. This study was aimed at characterizing non-subtype C HIV type 1 viruses from Cape Town, South Africa. This will greatly enrich our knowledge of other viral subtypes that are becoming more and more important in the South African setting. 


\section{CHAPTER TWO - MATERIALS AND METHODS}

Table of Contents

Page

2.1 Reagents and equipment used in the study 53

2.2 Patient samples $\quad 55$

2.3 Amplification and sequencing of partial gag, pol and env fragments $\quad 57$

2.3.1 PCR amplification of partial gag, pol and env fragments $\quad 57$

2.3.2 Gel electrophoresis and sample clean-up of PCR fragments 58

2.3.3 Sequencing of partial gag, pol and env PCR fragments $\quad 60$

2.4 HIV-1 subtyping of partial gag, pol and env sequences using

REGA and jpHMM online tools

62

2.5 Multiple alignments of the partial gag, pol and env sequences 62

2.6 Construction of NJ phylogenetic trees using MEGA 63

2.7 Amplification and sequencing of NFLG's from 4 samples 63

2.7.1 PCR amplification of the $9.2 \mathrm{kbp}$ HIV-1 genome 63

2.7.2 Amplification of the HIV genome in four overlapping fragments 66

2.7.3 Gel electrophoresis and clean-up of NFLG PCR $\begin{array}{ll}\text { fragments } & 70\end{array}$

$\begin{array}{ll}\text { 2.7.4 Sequencing of NFLG PCR fragments } & 70\end{array}$

2.8 Phylogenetic analysis of near full-length genome sequences 71

2.8.1 Subtyping with REGA and jpHMM tools $\quad 72$

2.8.2 Construction of a multiple alignment of NFLG sequences $\quad 72$

$\begin{array}{ll}2.8 .3 \text { Construction of phylogenetic trees } & 72\end{array}$

2.9 Detection of recombinant viruses using RIP and Simplot 73

2.10 Phylogenetic analysis of non-recombinant NFLG sequences $\quad 74$ 


\section{CHAPTER TWO - MATERIALS AND METHODS}

The experimental procedures used in this study are illustrated in this chapter. The materials and sampling methodology used will be described, followed by the experimental procedures used for the characterization of subgenomic and near full-length sequences (Figure 2.1).

\section{Experimental Methodology}

Tygerberg Virology Databank (400 V3 sequences)

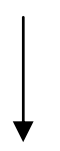

12 Samples were chosen [11 non-subtype $C$ and 1 subtype $C$ isolate]

Amplification and sequencing of gag p24, pol-integrase and env gp41 regions

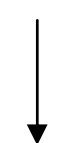

Subtyping of subgenomic regions and tree construction
Four samples were chosen for near full-length characterization

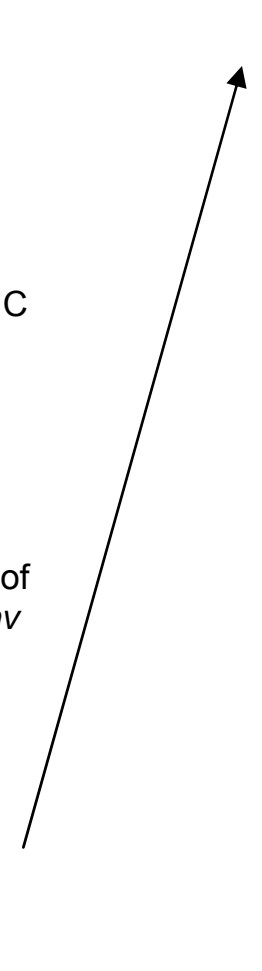

Amplification and sequencing (ABI $3130 \times 1$ automated DNA sequencer) of near-full length genomes

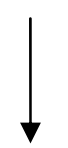

Phylogenetic analysis of near fulllength sequences

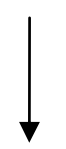

- Subtyping

- Tree construction

- Analysis of non recombinant isolates
- Recombination identification

Figure 2.1: A flow diagram summarizing the methodology used in the study.

Briefly, twelve samples were selected based on previous data that was generated within the department. Subgenomic regions of these 12 samples were amplified and directly sequenced. HIV subtyping and phylogenetic analysis was then performed on the sequenced data. From the data that was gathered, four samples were chosen for full or near full-length genomic characterization. After amplification and sequencing the sequenced data were 
analyzed with the use of phylogenetic methods, such as tree construction, subtyping, and recombination identification.

\subsection{Reagents and equipment used in the study}

All the materials and equipment that were used for the characterization of samples are described. A brief summary of all the equipment that was used are summarized in Table 2.1. The symbols ${ }^{\circledR}$ and ${ }^{\mathrm{TM}}$ indicate that the particular product are either a registered trademark or trademark of the suppliers.

Table 2.1: Equipment used to perform sample analysis.

\begin{tabular}{|l|l|l|}
\hline \multicolumn{1}{|c|}{ Equipment } & \multicolumn{1}{c|}{ Supplier } & \multicolumn{1}{c|}{ Location } \\
\hline Eppendorf Centrifuge 5417C & Eppendorf & Hamburg, Germany \\
\hline Eppendorf Centrifuge 5415D & Eppendorf & Hamburg, Germany \\
\hline GeneAMP ${ }^{\circledR}$ 9700 PCR system & Applied BioSystems & California, USA \\
\hline Hoefer EPS 2 A 200, Power Pack & $\begin{array}{l}\text { Pharmacla } \\
\text { Biotechnologies }\end{array}$ & San Francisco, CA, USA \\
\hline NanodropTM ND 1000 & $\begin{array}{l}\text { Nanodrop Technologies } \\
\text { Inc. }\end{array}$ & Delaware, USA \\
\hline SyngeneTM GeneGenius Computer System & Synoptics Ltd. & Cambridge, UK \\
\hline Vortex Mixer VM 300 & Gemmy Industrial Corp. & Taipei, Taiwan \\
\hline ABI 3130 xl Automated DNA sequencer & Applied BioSystems & California, USA \\
\hline
\end{tabular}

The chemicals and commercial products used in the study, and the various software packages used for sequence analysis are summarized in Tables 2.2 and 2.3 respectively. 
Table 2.2: List of chemicals and commercial products used in the study.

\begin{tabular}{|l|l|l|l|}
\hline \multicolumn{1}{|c|}{ Product } & \multicolumn{1}{c|}{ Company } & \multicolumn{1}{c|}{ Location } & \multicolumn{1}{c|}{ Catalogue Number } \\
\hline $\begin{array}{l}\text { Big Dye }{ }^{\circledR} \text { Terminator v 3.1 Cycle } \\
\text { Sequencing Kit }\end{array}$ & Applied BioSystems & Foster City, CA, USA & 0211005 \\
\hline Half Dye Mix & Bioline & London, UK & BIO-36026 \\
\hline GoTaq® DNA Polymerase & Promega & Madison, WI, USA & M 8305 \\
\hline Expand dNTP pack & Roche Diagnostics & Mannheim, Germany & 11681834001 \\
\hline Wizard SV Gel \& PCR Clean-up kit & Promega & Madison, WI, USA & A 9281 \\
\hline dNTP's & Roche Diagnostics & Mannheim, Germany & 11636103001 \\
\hline Ethidium bromide & Promega & Madison, WI, USA & H 5041 \\
\hline Nuclease Free Water & Promega & Madison, WI, USA & P 1193 \\
\hline Molecular grade Agarose & Pritehead Scientific (Pty) Ltd. & Brackenfell, Cape Town, RSA & \# D1 - LE \\
\hline I kbp DNA Bench Top Ladder & Promega & Madison, WI, USA & G7541 \\
\hline Blue/Orange Loading Dye & Promega & Madison, WI, USA & G1881 \\
\hline PCR Product Pre-Sequencing Kit & USB Corporation & Cleveland, Ohio, USA & 70995 \\
\hline
\end{tabular}


Table 2.3: Software packages used in the analysis of sequenced data.

\begin{tabular}{|c|c|}
\hline Software package & Reference / Licensed Company \\
\hline Sequencher 4.8 & Gene Codes Corporation, Ann Arbor, MI, USA \\
\hline Sequence Scanner v 1.0 & Applied Biosystems, Foster City, CA, USA \\
\hline Clustal X & Thompson $^{\odot}$ et al, 1997 \\
\hline DNAMAN v 4.0 & Lynnon BioSoft $^{\odot} 1994-1997$ \\
\hline BioEdit v 5.0.9 & Hall $^{\odot}, 2001$ \\
\hline Tree view 1.6 .6 & Page $^{\circledR}, 2001$ \\
\hline MEGA v 4.1 & Tamura et al, 2007 \\
\hline Simplot v 3.5 .1 & Stuart C Ray, Copyright ${ }^{\circledR} 1997-2003$ \\
\hline PAUP* $^{*} .0 \mathrm{~b} 10$ & Swofford, 2002 \\
\hline PhyML 3.0 & Guindon and Gascuel, 2003 \\
\hline Geneious 4 & Biomatters Ltd., Auckland, New Zealand \\
\hline FigTree v 1.1 .2 & Rambaut A, Institute of Evolutionary Biology, University of Edinburgh \\
\hline
\end{tabular}




\subsection{Patient samples}

From the data of a previous study that was done within the department, that characterized the $\mathrm{V} 3$ region of 410 samples [Jacobs et al, submitted], 11 samples were chosen. The study identified 36 out of 410 samples $(8.8 \%)$ as non-subtype C HIV-1 isolates but due to the limited amount of DNA available only 12 samples were chosen for further characterization. Ten of these samples were suspected to be non-subtype C HIV-1. The other sample was a known subtype $C$ viral isolate, which was included for control purposes. A known subtype $B$ isolate which was isolated from a homosexual male in the mid 1980's was also included for control purposes. A brief summary of the patient information are listed in Table 2.4 .

Table 2.4: Patient samples and demographics.

\begin{tabular}{|c|c|c|c|c|c|c|}
\hline Sample & $\begin{array}{l}\text { Race and } \\
\text { Gender }\end{array}$ & $\begin{array}{l}\text { Year of } \\
\text { Birth }\end{array}$ & $\begin{array}{l}\text { Country of } \\
\text { infection }\end{array}$ & Symptoms & $\begin{array}{c}\text { CD4 } \\
\text { Cells/ } \mu l\end{array}$ & $\begin{array}{c}\text { ARV } \\
\text { treatment }\end{array}$ \\
\hline R 84 & $\begin{array}{l}\text { Caucasian } \\
\text { male }\end{array}$ & ND & SA & Asymptomatic & NA & No \\
\hline TV 86 & African Male & 1965 & SA & Cryptococcal meningitis & 207 & No \\
\hline TV 101 & $\begin{array}{l}\text { African } \\
\text { Female }\end{array}$ & 1977 & SA & Asymptomatic & 2,000 & No \\
\hline TV 218 & $\begin{array}{l}\text { African } \\
\text { Female }\end{array}$ & 1975 & SA & Asymptomatic & NA & ND \\
\hline TV 239 & African Male & 1973 & SA & TB & 64 & No \\
\hline TV 314 & African Male & 1965 & SA & Asymptomatic & 229 & No \\
\hline TV 340 & African Male & 1963 & DRC & TB abdomen severe thrush & 3 & No \\
\hline TV 412 & African Male & 1955 & Kenya & Chronic staph skin sepses & 71 & No \\
\hline TV 441 & $\begin{array}{l}\text { African } \\
\text { Female }\end{array}$ & 1976 & SA & Asymptomatic & 178 & Yes \\
\hline TV 480 & $\begin{array}{l}\text { Coloured } \\
\text { Female }\end{array}$ & 1968 & SA & Pheumonia & NA & No \\
\hline TV 515 & $\begin{array}{l}\text { Coloured } \\
\text { Female }\end{array}$ & 1970 & SA & NA & NA & No \\
\hline TV 546 & $\begin{array}{l}\text { African } \\
\text { Female }\end{array}$ & 1970 & SA & NA & NA & ND \\
\hline
\end{tabular}

Key: SA (South Africa), DRC (Democratic Republic of the Congo), TV (Tygerberg Virology), NA (Not available), TB (Tuberculosis), staph (staphylococcal), and ND (No data) 
For DNA isolation, EDTA blood was centrifuged at 2,500 $\mathrm{xg}$ for $10 \mathrm{~min}$, to separate blood plasma and buffy coat cells. The buffy coat cells were then used to extract DNA with the use of the QIAamp ${ }^{\circledR}$ DNA Mini Kit (QIAGEN, Hilden, Germany), according to manufacturer's specifications. The DNA was eluted in $A E$ buffer and the concentrations were determined with the Nanodrop ${ }^{\text {TM }}$ ND 1000 (Nanodrop Technologies Inc., Delaware, USA)

\subsection{Amplification and sequencing of partial gag, pol and env fragments}

\subsubsection{PCR amplification of partial gag, pol and env fragments}

PCR's were performed on the gag p24, pol-integrase and env gp41 regions of the 12 samples. All the primers used and other relevant data are summarized in Table 2.5. All PCR's that was used for the amplification of subgenomic regions were performed with the GeneAmp PCR System 9700 thermal cycler (Applied BioSystems, CA, USA) with GoTaq DNA polymerase (Promega, Madison, WI, USA).

Briefly, the PCR methods and primers were adapted from Swanson et al, 2003. Both the prenested and nested PCR reactions for the gag, pol and env regions contained $0.2 \mathrm{mM}$ of dNTP's, $20 \mu \mathrm{M}$ of each primer, $1.5 \mathrm{mM}$ of $\mathrm{MgCl}_{2}$, and $1 \mathrm{U}$ of Taq polymerase in a total volume of $50 \mu \mathrm{l}$. The following cycling conditions were used for both the gag, pol and env PCR's: One cycle of denaturation at $94^{\circ} \mathrm{C}$ for 2 minutes; followed by 40 cycles of: denaturing at $94^{\circ} \mathrm{C}$ for 30 seconds, primer annealing for 30 seconds (Table 2.5), and elongation at $68^{\circ} \mathrm{C}$ for 1 minute; one final step of elongation at $68^{\circ} \mathrm{C}$ for 10 minutes and afterwards the samples were cooled down to $4^{\circ} \mathrm{C}$ until the PCR tubes were removed and stored at $4^{\circ} \mathrm{C}$. Two and a half micro liters of the prenested product was carried over to the nested reaction. 


\subsubsection{Gel electrophoresis and sample clean-up of PCR fragments}

PCR products of the gag p24, pol-integrase and env gp41 PCR's were run on $0.8 \%$ agarose gels $(10 \mathrm{~cm}$ long) at 50 Volts for 45 minutes in TAE buffer $(0.04$ $M$ TRIS-acetate \& $0.001 \mathrm{M}$ EDTA). After the samples migrated through the gels, the gels were stained with Ethidium Bromide $(0.5 \mu \mathrm{g} / \mathrm{ml})$ and exposed to UV light and photographs were taken.

Samples were then cleaned up with the use of the PCR Product PreSequencing Kit (USB Corporation, Cleveland, Ohio, USA). The kit uses two enzymes, Exonuclease I, which is responsible for removing any residual single-stranded primers or any other extraneous single-stranded DNA, and Shrimp Alkaline Phosphatase, which is responsible for the removing of any remaining dNTP's. The two enzymes were added to $8 \mu$ of amplified product and incubated at $37^{\circ} \mathrm{C}$ for 15 minutes. Samples were then heated at $80^{\circ} \mathrm{C}$ for $15 \mathrm{~min}$ to inactivate the enzymes.

The concentrations of the cleaned up DNA products were determined with the Nanodrop ${ }^{\text {TM }}$ ND 1000 (Nanodrop Technologies Inc., Delaware, USA). 
Table 2.5: List of different primers used in the amplification of the gag (p24), pol-integrase and the env (gp41) fragments.

\begin{tabular}{|c|c|c|c|c|c|c|c|}
\hline & Primers & Oligonucleotide sequences & $\mathrm{T}_{\mathrm{A}}\left({ }^{\circ} \mathrm{C}\right)$ & HXB2 Position & F/R & {$\left[\mathrm{MgCl}_{2}\right]$} & Size \\
\hline \multirow{2}{*}{ PN gag p24 } & p24-1 & AGYCAAAATTAYCCYATAGT & 45 & $1174-1194$ & $\mathrm{~F}$ & $1.5 \mathrm{mM}$ & \multirow{2}{*}{$671 \mathrm{bp}$} \\
\hline & p24-7 & CCCTGRCATGCTGTCATCA & 45 & $1844-1826$ & $\mathrm{R}$ & $1.5 \mathrm{mM}$ & \\
\hline \multirow{2}{*}{ N gag p24 } & $\mathrm{p} 24-2$ & AGRACYTTRAAYGCATGGGT & 50 & $1237-1256$ & $\mathrm{~F}$ & $1.5 \mathrm{mM}$ & \multirow{2}{*}{$485 \mathrm{bp}$} \\
\hline & $\mathrm{p} 24-6$ & TGTGWAGCTTGYTCRGCTC & 50 & $1721-1703$ & $\mathrm{R}$ & $1.5 \mathrm{mM}$ & \\
\hline \multirow{2}{*}{ PN pol-IN } & poli 5 & CACACAAAGGRATTGGAGGAAATG & 50 & $4162-4185$ & $\mathrm{~F}$ & $1.5 \mathrm{mM}$ & \multirow{2}{*}{$\begin{array}{c}1056 \\
\text { bp }\end{array}$} \\
\hline & poli 8 & TAGTGGGATGTGTACTTCTGAAC & 50 & $5217-5195$ & $\mathrm{R}$ & $1.5 \mathrm{mM}$ & \\
\hline \multirow{2}{*}{ N pol-IN } & poli 6 & ATACATATGRTGTTTTACTAARCT & 45 & $5130-5107$ & $\mathrm{R}$ & $1.5 \mathrm{mM}$ & \multirow{2}{*}{$945 \mathrm{bp}$} \\
\hline & poli 7 & AACAAGTAGATAAATTAGTCAGT & 45 & $4186-4208$ & $\mathrm{~F}$ & $1.5 \mathrm{mM}$ & \\
\hline \multirow{2}{*}{ PN env gp41 } & $\mathrm{JH} 38$ & GGTGARTATCCCTKCCTAAC & 50 & $8365-8346$ & $\mathrm{R}$ & $1.5 \mathrm{mM}$ & \multirow{2}{*}{$568 \mathrm{bp}$} \\
\hline & $\mathrm{JH} 41$ & TTATATAАТTСАСТTСТССААТT & 50 & $7775-7797$ & $\mathrm{~F}$ & $1.5 \mathrm{mM}$ & \\
\hline \multirow{2}{*}{ N env gp41 } & env $27 \mathrm{~F}$ & CTGGYATAGTGCARCARCA & 45 & $7861-7879$ & $\mathrm{~F}$ & $1.5 \mathrm{mM}$ & \multirow{2}{*}{$439 \mathrm{bp}$} \\
\hline & Menv 19R & AARCCTCCTACTATCATTATRA & 45 & $8299-8278$ & $\mathrm{R}$ & $1.5 \mathrm{mM}$ & \\
\hline
\end{tabular}

Key: PN (Prenested PCR), N (Nested PCR), TA (annealing temperature), F (Forward primer), R (Reverse primer), ${ }^{\circ} \mathrm{C}$ (degrees Celsius), bp (base pairs), IN (Integrase), and $\mathrm{mM}$ (millimoles per liter) 


\subsubsection{Sequencing of partial gag, pol and env PCR fragments}

Amplified gag p24, pol-integrase and env gp41 fragments were all directly sequenced with the use of the primers listed in Table 2.6. All primers were described by Swanson et al, 2003 except for primer FGF 46 [Fong et al, 1996]. Samples TV101 and TV218 have already been characterized and was not sequenced with the other samples [Personal Communication, $S$ Engelbrecht]. The BigDye ${ }^{\mathrm{TM}}$ Terminator cycle sequencing ready reaction kit (Applied Biosystems, Foster City, California, USA) was used for the sequencing reactions.

Every sequencing reaction contained: approximately $50 \mathrm{ng}$ of the purified PCR product, $5 \mathrm{pmol}$ of sequencing primer, $1.3 \mu \mathrm{l}$ of Big Dye terminator enzyme mix, and $2.7 \mu \mathrm{l}$ of Half Dye (Bioline, London, United Kingdom). Nuclease free water was added to the reaction mix to give a final volume of 10 $\mu l$. Each sequencing reaction was performed under the following conditions: 25 cycles of denaturation at $96^{\circ} \mathrm{C}$ for 10 seconds, primer annealing for 5 seconds and an elongation step at $60^{\circ} \mathrm{C}$ for 4 minutes. Afterwards the samples were cooled down to $4^{\circ} \mathrm{C}$ and sent to the Central Analytical Facility of the University of Stellenbosch where they could be cleaned-up and run on the $A B|3130 x|$ automated DNA sequencer.

The trace data files were received from the Central Analytical Facility and were imported into Sequencer 4.7 (Gene Codes Corporation, Ann Arbor., Michigan, USA) and assembled into contiguous fragments. After the assembled fragments were proofread they were exported in a text file (.txt) and labeled for later use. 
Table 2.6: List of different sequencing primers for the gag p24, pol-integrase and the env gp41 fragments.

\begin{tabular}{|c|c|c|c|c|c|}
\hline \multicolumn{6}{|c|}{ gag p24 Sequences } \\
\hline Primer & Oligo nucleotide sequence & Bases & F / R & HXB2 Position & $\mathrm{T}_{\mathrm{A}}\left({ }^{\circ} \mathrm{C}\right)$ \\
\hline p24-2 & AGRACYTTRAAYGCATGGGT & 20 & $\mathrm{~F}$ & $1237-1257$ & 50 \\
\hline p24-6 & TGTGWAGCTTGYTCRGCTC & 19 & $R$ & $1703-1721$ & 50 \\
\hline \multicolumn{6}{|c|}{ pol-integrase Sequences } \\
\hline Primer & Oligo nucleotide sequence & Bases & F / R & HXB2 Position & $\mathrm{T}_{\mathrm{A}}\left({ }^{\circ} \mathrm{C}\right)$ \\
\hline poli7 & AACAAGTAGATAAATTAGTCAGT & 23 & $\mathrm{~F}$ & $4186-4209$ & 45 \\
\hline FGF-46 & GCATTCCСТАCAATCCCCAAAG & 22 & $\mathrm{~F}$ & $4648-4670$ & 55 \\
\hline poli10b & TATTCATAGATTCYACTACTCCTTG & 25 & $\mathrm{R}$ & $4695-4670$ & 45 \\
\hline poli6 & ATACATATGRTGTTTTACTAARCT & 24 & $\mathrm{R}$ & $5130-5106$ & 45 \\
\hline \multicolumn{6}{|c|}{ env gp41 Sequences } \\
\hline Primer & Oligo nucleotide sequence & Bases & F / R & HXB2 Position & $\mathrm{T}_{\mathrm{A}}\left({ }^{\circ} \mathrm{C}\right)$ \\
\hline env $27 \mathrm{~F}$ & CTGGYATAGTGCARCARCA & 19 & $\mathrm{~F}$ & $7861-7880$ & 50 \\
\hline Menv19R & AARCCTCCTACTATCATTATRA & 22 & $\mathrm{R}$ & $8299-8277$ & 45 \\
\hline
\end{tabular}

Key: $\mathrm{T}_{\mathrm{A}}$ (annealing temperature), $\mathrm{F}$ (Forward primer), $\mathrm{R}$ (Reverse Primer), and ${ }^{\circ} \mathrm{C}$ (degrees Celsius) 


\subsection{HIV-1 subtyping of partial gag, pol and env sequences using REGA and jpHMM online tools}

HIV subtyping was performed on all the sequenced samples to establish the genomic variation of our cohort of 12 samples. Two different online subtyping tools were used for this purpose: the REGA subtyping tool (http://www.bioafrica.net/virus-genotype/html/subtyping.html) and the jumping profile Hidden Markov Model or jpHMM (http://jphmm.gobics.de/) which is also accessible form the LANL webpage (http://www.hiv.lanl.gov/). The REGA subtyping tool is an easy online method of subtyping full-length or subgenomic fragments by combining different phylogenetic analytical approaches with bootscanning methods [de Oliveira et al, 2005].

The jpHMM method uses a jumping alignment approach, first proposed by Spang and co-workers [Spang et al, 2002], for the subtyping of sequence fragments or the identification of recombinant viruses. Instead of a query sequence $(X)$ being compared with a multiple alignment $(Y)$, the query sequence $(X)$ is compared and aligned to individual sequences from the alignment. In the case of recombination events the query sequence $(X)$ can then jump between different sequences of the multiple alignment as a sliding window moves over the alignment. This approach also makes the identification of particular breakpoint within a recombinant isolate much easier [Schultz et al, 2006; Zhang et al, 2006].

\subsection{Multiple alignments of the partial gag, pol and env sequences}

Before multiple alignments could be constructed, reference sequences were obtained from the LANL database (http://www.hiv.lanl.gov/). For the alignment of gag p24 fragments, sequences of 485 bp and stretching from $1237-1721$ (relative to HXB2) were obtained. Reference sequences corresponding to the same length and location of the pol-integrase and env gp41 PCR fragments, stretching from 4186 - 5130 (944 bp) and 7861 - 8299 (438 bp) relative to HXB2 respectively, were acquired. After the reference sequences were obtained multiple alignments were constructed with the use of Clustal $X \vee 1.81$ 
[Thompson ${ }^{\odot}$ et al, 1997]. All alignments were manually checked and improved, where possible, with BioEdit v 5.09 [Hall $^{\odot}$, 2001].

The isolate N.CM. 95. YBF30. AJ 006022 was included as an outgroup in the alignments. Every name in the LANL database is specifically classified and annotated. For example in strain N.CM. 95. YBF30. AJ006022, the N stands for Group N, CM the country of origin (Cameroon), 95 the year the sample was collected (1995), YBF30 is the name of the virus strain and AJ006022 is the GenBank Accession number.

\subsection{Construction of NJ phylogenetic trees using MEGA}

After the alignments were done and manually inspected, Neighbor-Joining phylogenetic trees were constructed. Adjusted multiple alignment files were imported into MEGA $\vee 4.1$ [Tamura et al, 2007] where the alignment files (.aln or .pir) were converted into MEGA format (.meg). The MEGA files (.meg) were then opened in MEGA and NJ-trees [Saitou and Nei, 1987] were constructed with the use of the Kimura 2 parameter method of nucleotide substitution [Kimura, 1980]. Bootstrap analysis [Felsenstein, 1985] was also performed on the trees to confer statistical significance, with a total of a 1,000 bootstrap replicates for each dataset.

\subsection{Amplification and sequencing of NFLG's from 4 samples}

After data analysis of the gag pol and env regions, full- or near full-length genome characterization of four of the twelve samples (R84, TV239, TV314 and TV412) were attempted. The entire genomes of the four different samples were first amplified in a single amplification assay to obtain $9.2 \mathrm{kbp}$ products.

\subsubsection{PCR amplification of the 9.2 kbp HIV-1 genome}

Briefly, a prenested and nested PCR were carried out with the Expand long range dNTP pack (Roche Diagnostics, Mannheim, Germany) to obtain nearfull length genome amplification of HIV samples (Figure 2.2). 
Prenested PCR

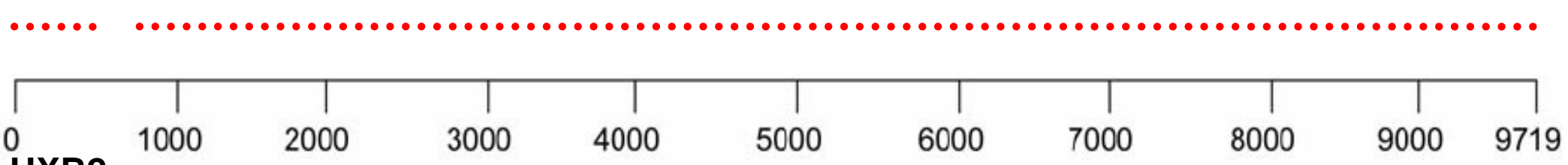

\section{HXB2}

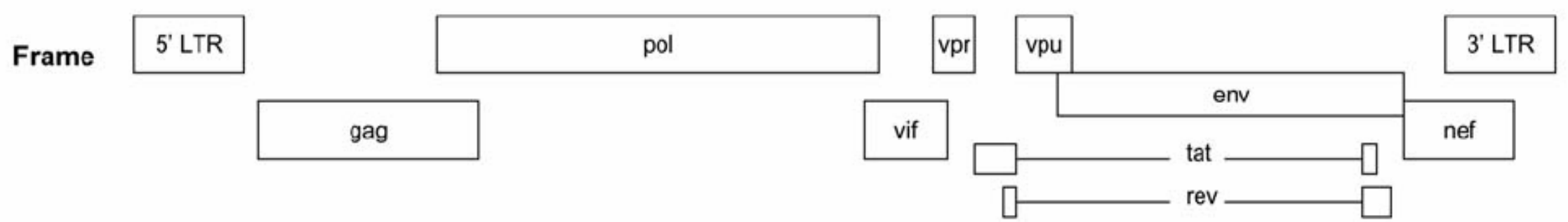

Figure 2.2: Schematic diagrams of the full genome amplification of an HIV isolate using a long PCR amplification assay. The prenested and the nested PCR are indicated on the diagram. Both forward and reverse primers as well as their coordinates (relative to HXB2) are indicated. 
Each PCR reaction contained $0.2 \mathrm{mM}$ of dNTP's, $20 \mu \mathrm{M}$ of each primer, $12 \%$ DMSO solution and $5 \mathrm{U}$ of DNA polymerase in a total volume of $50 \mu \mathrm{l}$. The buffer that was used already contained $\mathrm{MgCl}_{2}$. The rest of the volume was made up to $50 \mu \mathrm{l}$ with nuclease free water (Promega, Madison, WI, USA).

The following cycling conditions were used for the prenested and nested amplification assays: one cycle of denaturation at $92^{\circ} \mathrm{C}$ for 2 minutes; followed by 10 cycles of denaturation at $92^{\circ} \mathrm{C}$ for 10 seconds, primer annealing for 30 seconds (Table 2.7), and elongation at $68^{\circ} \mathrm{C}$ for 10 minutes; which was followed by 30 cycles of; denaturation at $92^{\circ} \mathrm{C}$ for 10 seconds, primer annealing for 30 seconds, and elongation at $68^{\circ} \mathrm{C}$ for 10 minutes plus 20 seconds for each consecutive cycle, final elongation at $68^{\circ} \mathrm{C}$ for 10 minutes and afterwards the samples were cooled down to $4^{\circ} \mathrm{C}$ until the PCR reactions were collected. Five micro liters of the prenested product were carried over to the nested reaction.

Table 2.7: Primers that were used for the amplification of $9.2 \mathrm{kbp}$ fragments.

\begin{tabular}{|c|c|c|c|c|c|c|}
\hline & \multicolumn{6}{|c|}{ Full genome Primers } \\
\hline & Primer & Oligo nucleotide sequence & $\mathbf{F} / \mathbf{R}$ & HXB2 Position & $\mathrm{T}_{\mathrm{A}}\left({ }^{\circ} \mathrm{C}\right)$ & Size \\
\hline \multirow{2}{*}{ PN } & $\operatorname{MSF} 12^{\mathrm{A}}$ & AAATCTCTAGCAGTGGCGCCCCGAACAG & $\mathrm{F}$ & $623-649$ & 55 & \multirow{2}{*}{$\begin{array}{l}9.17 \\
\mathrm{kbp}\end{array}$} \\
\hline & $\mathrm{MSR}^{\mathrm{A}}$ & GCACTCAAGGCAAGCTTTATTGAGGCT & $\mathrm{R}$ & $9797-9823$ & 55 & \\
\hline \multirow{2}{*}{$\mathbf{N}$} & UP1A ${ }^{B}$ & AGTGGCGCCCGAACAGG & $\mathrm{F}$ & $634-650$ & 60 & \multirow{2}{*}{$\begin{array}{l}9.09 \\
\text { kbp }\end{array}$} \\
\hline & $\operatorname{LOW}^{\mathrm{B}}$ & TGAGGCTTAAGCAGTGGGTTTC & $\mathrm{R}$ & $9706-9727$ & 60 & \\
\hline
\end{tabular}

Key: PN (Prenested PCR), N (Nested PCR), F (Forward primer), R (Reverse primer), ${ }^{\circ} \mathrm{C}$ (degrees Celsius), and kbp (kilo base pairs) [ ${ }^{A}$ Rodenburg et al, 2001; ${ }^{B}$ Mwaengo and Novembre, 1998].

The following cycling conditions were used for the prenested and nested amplification assays: one cycle of denaturation at $92^{\circ} \mathrm{C}$ for 2 minutes; followed by 10 cycles of denaturation at $92^{\circ} \mathrm{C}$ for 10 seconds, primer annealing for 30 seconds (Table 2.7), and elongation at $68^{\circ} \mathrm{C}$ for 10 minutes; which was followed by 30 cycles of; denaturation at $92^{\circ} \mathrm{C}$ for 10 seconds, primer annealing for 30 seconds, and elongation at $68^{\circ} \mathrm{C}$ for 10 minutes plus 20 seconds for each consecutive cycle, final elongation at $68^{\circ} \mathrm{C}$ for 10 minutes and afterwards the samples were cooled down to $4^{\circ} \mathrm{C}$ until the PCR reactions 
were collected. Five micro liters of the prenested product were carried over to the nested reaction.

\subsubsection{Amplification of the HIV genome in four overlapping fragments}

The original proviral DNA was then used for the amplification of a near-full length genome consisting of four overlapping PCR fragments (Figure 2.3) spanning the entire genome of the four isolates.

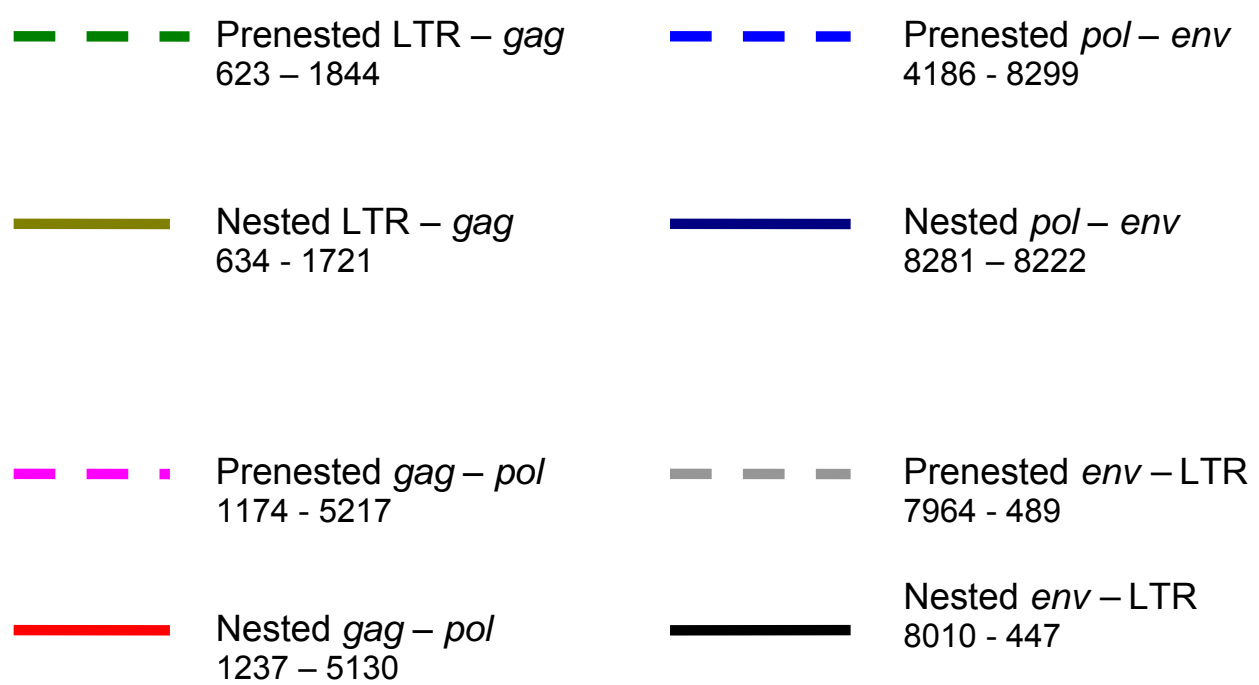
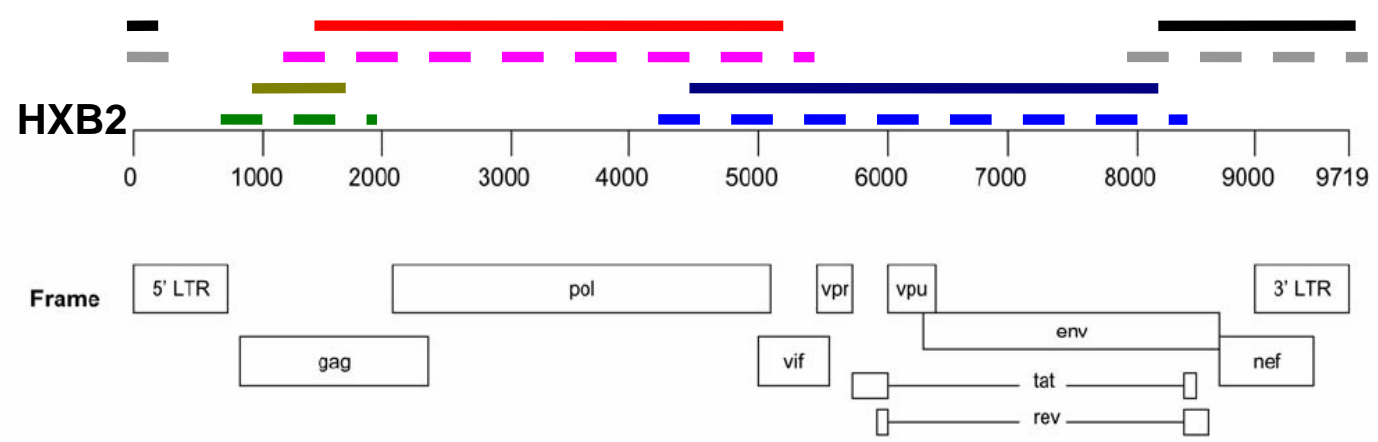

Figure 2.3: Schematic diagrams of the full genome amplification of an HIV isolate in four overlapping fragments. The prenested and nested amplifications and their approximate location (relative to HXB2) are indicated. All dotted lines indicate prenested reactions and all solid lines nested reactions. 
Table 2.8: Primers that were used to PCR the overlapping fragments (LTR-gag, gag-pol, pol-env, and env-LTR).

\begin{tabular}{|c|c|c|c|c|c|c|c|}
\hline & \multicolumn{7}{|c|}{ LTR-gag Primers } \\
\hline & Primer & Oligo nucleotide sequence & F/R & HXB2 Position & $\mathrm{T}_{\mathrm{A}}\left({ }^{\circ} \mathrm{C}\right)$ & Size & Ref \\
\hline \multirow{2}{*}{ PN } & MSF12 & AAATCTCTAGCAGTGGCGCCCCGAACAG & $\mathrm{F}$ & $623-649$ & 52 & \multirow{2}{*}{$\begin{array}{l}1.21 \\
\mathrm{kbp}\end{array}$} & Rodenburg et al, 2001 \\
\hline & P24-7 & CCCTGRCATGCTGTCATCA & $\mathrm{R}$ & $1826-1844$ & 52 & & Swanson et al, 2003 \\
\hline \multirow{4}{*}{$\mathbf{N}$} & UP1A & AGTGGCGCCCGAACAGG & $\mathrm{F}$ & $634-650$ & 50 & \multirow{2}{*}{$\begin{array}{l}1.09 \\
\mathrm{kbp}\end{array}$} & Mwaengo and Novembre, 1998 \\
\hline & P24-6 & TGTGWAGCTTGYTCRGCTC & $\mathrm{R}$ & $1703-1721$ & 50 & & Swanson et al, 2003 \\
\hline & \multicolumn{7}{|c|}{ gag-pol Primers } \\
\hline & Primer & Oligo nucleotide sequence & $\mathbf{F} / \mathbf{R}$ & HXB2 Position & $\mathrm{T}_{\mathrm{A}}\left({ }^{\circ} \mathrm{C}\right)$ & Size & Ref \\
\hline \multirow{2}{*}{ PN } & p24-1 & AGYCAAAATTAYCCYATAGT & $\mathrm{F}$ & $1174-1193$ & 45 & \multirow{2}{*}{$\begin{array}{l}4.04 \\
\mathrm{kbp}\end{array}$} & \multirow{4}{*}{ Swanson et al, 2003} \\
\hline & poli 8 & TAGTGGGATGTGTACTTCTGAAC & $\mathrm{R}$ & $5195-5217$ & 45 & & \\
\hline \multirow{2}{*}{$\mathbf{N}$} & $\mathrm{p} 24-2$ & AGRACYTTRAAYGCATGGGT & $\mathrm{F}$ & $1237-1256$ & 50 & \multirow{2}{*}{$\begin{array}{l}3.89 \\
\text { kbp }\end{array}$} & \\
\hline & poli 6 & ATACATATGRTGTTTTACTAARCT & $\mathrm{R}$ & $5107-5130$ & 50 & & \\
\hline
\end{tabular}

Key: PN (Prenested PCR), N (Nested PCR), LTR (Long terminal repeat), ${ }^{\circ} \mathrm{C}$ (degrees Celsius), $\mathrm{T}_{\mathrm{A}}$ (annealing temperature), $\mathrm{F}$ (Forward primer), R (Reverse primer), Ref (Reference) and kbp (kilo base pairs) 
Table 2.8 continued: Primers that were used to PCR the overlapping fragments (LTR-gag, gag-pol, pol-env, and env-LTR).

\begin{tabular}{|c|c|c|c|c|c|c|c|}
\hline & \multicolumn{7}{|c|}{ pol-env Primers } \\
\hline & Primer & Oligo nucleotide sequence & F/R & HXB2 Position & $\mathrm{T}_{\mathrm{A}}\left({ }^{\circ} \mathrm{C}\right)$ & Size & Ref \\
\hline \multirow{2}{*}{ PN } & poli 7 & AACAAGTAGATAAATTAGTCAGT & $\mathrm{F}$ & $4186-4208$ & 45 & \multirow{2}{*}{$\begin{array}{l}4.11 \\
\mathrm{kbp}\end{array}$} & \multirow{4}{*}{ Swanson et al, 2003} \\
\hline & Menv 19 & AARCCTCCTACTATCATTATRA & $\mathrm{R}$ & $8278-8299$ & 45 & & \\
\hline \multirow{4}{*}{$\mathbf{N}$} & PPF17 & AATTGGAGAGCAATGGCTAGTGA & $\mathrm{F}$ & $4281-4303$ & 50 & \multirow{2}{*}{$\begin{array}{l}3.94 \\
\mathrm{kbp}\end{array}$} & \\
\hline & LP 7728 & ССАCTTGTCCAATGCCAATAAGTCTTGT & $\mathrm{R}$ & $8195-8222$ & 50 & & \\
\hline & \multicolumn{7}{|c|}{ env-LTR Primers } \\
\hline & Primer & Oligo nucleotide sequence & F/R & HXB2 Position & $\mathrm{T}_{\mathrm{A}}\left({ }^{\circ} \mathrm{C}\right)$ & Size & Ref \\
\hline \multirow{2}{*}{ PN } & $7496 \mathrm{~F}$ & CCTKGCYCTGGAAAGATACCTA & $\mathrm{F}$ & $7964-7985$ & 52 & \multirow{2}{*}{$\begin{array}{l}1.70 \\
\mathrm{kbp}\end{array}$} & \multirow{4}{*}{$\begin{array}{l}\text { Personal Communication John } \\
\text { Hackett }\end{array}$} \\
\hline & 9131R-2 & CTCYCAGGCTCARATCTGGTC & $\mathrm{R}$ & $468-489$ & 52 & & \\
\hline \multirow{2}{*}{$\mathbf{N}$} & $7542 \mathrm{~F}$ & TGGGGCTGCTCTGGAAAACT & $\mathrm{F}$ & $8010-8029$ & 50 & \multirow{2}{*}{$\begin{array}{l}1.64 \\
\mathrm{kbp}\end{array}$} & \\
\hline & 9110R-2 & CAAGAGAGACCCAGTACAG & $\mathrm{R}$ & $447-465$ & 50 & & \\
\hline
\end{tabular}

Key: PN (Prenested PCR), N (Nested PCR), LTR (Long terminal repeat), ${ }^{\circ} \mathrm{C}$ (degrees Celsius), $\mathrm{T}_{\mathrm{A}}$ (annealing temperature), $\mathrm{F}$ (Forward primer), $\mathrm{R}($ Reverse primer), Ref (Reference) and kbp (kilo base pairs) 
Table 2.9: PCR cycling condition of the four overlapping fragments.

\begin{tabular}{|c|c|c|c|c|c|c|c|c|c|}
\hline \multirow{3}{*}{ Cycle(s) } & \multirow{3}{*}{ Reaction } & \multicolumn{8}{|c|}{ Temperatures and Time } \\
\hline & & \multicolumn{2}{|c|}{ LTR-gag PCR } & \multicolumn{2}{|c|}{ gag-pol PCR } & \multicolumn{2}{|c|}{ pol-env PCR } & \multicolumn{2}{|c|}{ env-LTR PCR } \\
\hline & & $\begin{array}{c}\text { Prenested } \\
\text { PCR }\end{array}$ & $\begin{array}{c}\text { Nested } \\
\text { PCR }\end{array}$ & $\begin{array}{l}\text { Prenested } \\
\text { PCR }\end{array}$ & $\begin{array}{l}\text { Nested } \\
\text { PCR }\end{array}$ & $\begin{array}{l}\text { Prenested } \\
\text { PCR }\end{array}$ & $\begin{array}{l}\text { Nested } \\
\text { PCR }\end{array}$ & $\begin{array}{c}\text { Prenested } \\
\text { PCR }\end{array}$ & $\begin{array}{c}\text { Nested } \\
\text { PCR }\end{array}$ \\
\hline $1 x$ & $\begin{array}{l}\text { Template } \\
\text { denaturing }\end{array}$ & $94^{\circ} \mathrm{C}, 2 \mathrm{~m}$ & $94^{\circ} \mathrm{C}, 2 \mathrm{~m}$ & $94^{\circ} \mathrm{C}, 2 \mathrm{~m}$ & $94^{\circ} \mathrm{C}, 2 \mathrm{~m}$ & $94^{\circ} \mathrm{C}, 2 \mathrm{~m}$ & $94^{\circ} \mathrm{C}, 2 \mathrm{~m}$ & $94^{\circ} \mathrm{C}, 2 \mathrm{~m}$ & $94^{\circ} \mathrm{C}, 2 \mathrm{~m}$ \\
\hline \multirow{3}{*}{$40 x$} & $\begin{array}{l}\text { Template } \\
\text { denaturing }\end{array}$ & $94^{\circ} \mathrm{C}, 30 \mathrm{~s}$ & $94^{\circ} \mathrm{C}, 30 \mathrm{~s}$ & $94^{\circ} \mathrm{C}, 30 \mathrm{~s}$ & $94^{\circ} \mathrm{C}, 30 \mathrm{~s}$ & $94^{\circ} \mathrm{C}, 30 \mathrm{~s}$ & $94^{\circ} \mathrm{C}, 30 \mathrm{~s}$ & $94^{\circ} \mathrm{C}, 30 \mathrm{~s}$ & $94^{\circ} \mathrm{C}, 30 \mathrm{~s}$ \\
\hline & $\begin{array}{l}\text { Primer } \\
\text { Annealing }\end{array}$ & $52^{\circ} \mathrm{C}, 30 \mathrm{~s}$ & $51^{\circ} \mathrm{C}, 30 \mathrm{~s}$ & $45^{\circ} \mathrm{C}, 30 \mathrm{~s}$ & $45^{\circ} \mathrm{C}, 30 \mathrm{~s}$ & $45^{\circ} \mathrm{C}, 30 \mathrm{~s}$ & $50^{\circ} \mathrm{C}, 30 \mathrm{~s}$ & $52^{\circ} \mathrm{C}, 30 \mathrm{~s}$ & $50^{\circ} \mathrm{C}, 30 \mathrm{~s}$ \\
\hline & Elongation & $68^{\circ} \mathrm{C}, 90 \mathrm{~s}$ & $68^{\circ} \mathrm{C}, 90 \mathrm{~s}$ & $68^{\circ} \mathrm{C}, 4 \mathrm{~m}$ & $68^{\circ} \mathrm{C}, 4 \mathrm{~m}$ & $68^{\circ} \mathrm{C}, 4 \mathrm{~m}$ & $68^{\circ} \mathrm{C}, 4 \mathrm{~m}$ & $68^{\circ} \mathrm{C}, 2 \mathrm{~m}$ & $68^{\circ} \mathrm{C}, 2 \mathrm{~m}$ \\
\hline $1 \mathrm{x}$ & Final Elongation & $68^{\circ} \mathrm{C}, 10 \mathrm{~m}$ & $68^{\circ} \mathrm{C}, 10 \mathrm{~m}$ & $68^{\circ} \mathrm{C}, 10 \mathrm{~m}$ & $68^{\circ} \mathrm{C}, 10 \mathrm{~m}$ & $68^{\circ} \mathrm{C}, 10 \mathrm{~m}$ & $68^{\circ} \mathrm{C}, 10 \mathrm{~m}$ & $68^{\circ} \mathrm{C}, 10 \mathrm{~m}$ & $68^{\circ} \mathrm{C}, 10 \mathrm{~m}$ \\
\hline $1 \mathrm{x}$ & Storing & $4^{\circ} \mathrm{C}$, Indef & $4^{\circ} \mathrm{C}$, Indef & $4^{\circ} \mathrm{C}$, Indef & $4^{\circ} \mathrm{C}$, Indef & $4^{\circ} \mathrm{C}$, Indef & $4^{\circ} \mathrm{C}$, Indef & $4^{\circ} \mathrm{C}$, Indef & $4^{\circ} \mathrm{C}$, Indef \\
\hline
\end{tabular}

Key: $\mathrm{x}$ (times), PCR (polymerase chain reaction), ${ }^{\circ} \mathrm{C}$ (degrees Celsius), $\mathrm{m}$ (minutes), s (seconds), and Indef (indefinitely). 
Each PCR reaction contained $0.2 \mathrm{mM}$ of dNTP's, $20 \mu \mathrm{M}$ of each primer, 1.5 $\mathrm{mM}$ of $\mathrm{MgCl}_{2}$, and $1 \mathrm{U}$ of $\mathrm{Taq}$ polymerase in a total volume of $50 \mu \mathrm{l}$. Five micro liters of the prenested product (ranging between 10 and $50 \mu \mathrm{g} / \mu \mathrm{l}$ ) was carried over to each of the nested reaction. Table 2.9 gives a brief summary of the cycling conditions that was used.

\subsubsection{Gel electrophoresis and clean-up of NFLG PCR fragments}

PCR products were run on $0.8 \%$ agarose gels $(10 \mathrm{~cm}$ in length) at 50 Volts for 45 minutes in TAE buffer (0.04 M TRIS-acetate \& $0.001 \mathrm{M}$ EDTA). A $1 \mathrm{kbp}$ molecular marker was run in parallel with all samples. After the samples migrated through the gels, the gels were stained with Ethidium Bromide (0.5 $\mu \mathrm{g} / \mathrm{ml}$ ) and exposed to UV light before photographs were taken.

The Wizard SV gel and PCR clean-up kit from Promega, Madison, Wisconsin, USA were used to purify the amplified products of any unwanted dNTP's or oligonucleotides. The concentrations of the cleaned up products were determined with the Nanodrop ${ }^{\mathrm{TM}}$ ND 1000 (Nanodrop Technologies Inc., Delaware, USA) after they were eluted from the spin column.

\subsubsection{Sequencing of NFLG PCR fragments}

The various amplified products were directly sequenced by employing primer walking techniques. Appropriate primers were chosen for every $400-500$ base pairs in both the forward and reverse directions (John Hackettt, personal communication). All the sequencing primers use in the sequencing of each isolate is listed in Tables 6.4-6.7 in Appendix C, Chapter 6.

The BigDye ${ }^{\mathrm{TM}}$ Terminator cycle sequencing ready reaction kit (Applied Biosystems, Foster City, California, USA) was used for the PCR based sequencing reactions. Approximately $50 \mathrm{ng}$ of the purified PCR product were used with, $5 \mathrm{pmol}$ of sequencing primer, $1.3 \mu \mathrm{l}$ of Big Dye terminator enzyme 
mix, and $2.7 \mu \mathrm{l}$ of Half Dye (Bioline, London, United Kingdom). Nuclease free water was added to the mix to give a final volume of $10 \mu$ of reaction mix. Each sequencing reaction were performed under the following sequencing cycling reaction conditions: 25 cycles of denaturization at $96^{\circ} \mathrm{C}$ for 10 seconds, primer annealing for 5 seconds and an elongation step at $60^{\circ} \mathrm{C}$ for 4 minutes. Afterwards the samples were cooled down to $4^{\circ} \mathrm{C}$ and sequenced at the Central Analytical Facility at the University of Stellenbosch, as described before. After the trace data files were recovered from the Central Analytical Facility they were imported into Sequencer 4.7 (Gene Codes Corporation, Ann Arbor., Michigan, USA) were they were assembled into contiguous fragments. After the assembled fragments were proofread they were exported as text files (.txt).

The contiguous fragments were also analyzed with the use of sequence tools such as the Gene Cutter tool of the LANL database [http://www.hiv.lanl.gov/]. The Gene Cutter tool [Goldman and Yang, 1994] is an online tool from the Los Alamos National Laboratory which can be used to identify the different genes within sequences, produce information on the amino acid sequence, and identify premature stop codons and sites with multi-state characters. Sequences can be submitted in an aligned or unaligned format.

\subsection{Phylogenetic analysis of near full-length genome sequences}

After all sequences were proof-read, quality controlled phylogenetic analysis could be conducted. Briefly, the DNA sequence fragments were used to perform subtyping to establish viral subtypes and/or recombinants. Multiple alignments were constructed and phylogenetic trees were drawn. Bootstrap analysis was performed on all trees for statistical purposes. Recombination identification was also performed to identify recombination events within isolates. Non-recombinant HIV isolates were also compared against samples of the same subtype for further in-depth analysis. 


\subsubsection{Subtyping with REGA and jpHMM tools}

All the near full-length fragments were submitted to two online subtyping tools; the jpHMM (http://jphmm.gobics.de/ which is also accessible at the LANL website http://hiv.lanl.gov.l) and the REGA subtyping tools (http://www.bioafrica.net/virus-genotype/html/subtyping.html).

\subsubsection{Construction of a multiple alignment of NFLG sequences}

Reference sequences were obtained from the LANL database before multiple alignments could be constructed. All non-recombinant subtypes of the reference set, as well as the most prominent recombinant viruses, were included in the datasets that was used for the various alignments. Due to the varying length of the different fragments reference sequences ranging from 1230 - 8700 (relative to the coordinates of the reference strain HXB2) were downloaded. Because no contiguous fragment could be obtained for TV239, reference sequences in two fragments stretching from 1246 - 5534 and 5908 9106 (relative to the coordinates of the reference strain HXB2) were downloaded. The reference strain N.CM.95.YBF30.AJ006022 was included in each dataset for the use as an outgroup.

Multiple alignments were then constructed with the use of Clustal $X \vee 1.81$ [Thompson ${ }^{\odot}$ et al, 1997]. The three isolates; R84, TV314, and TV412 were all aligned in a single alignment. Due to a gap of nearly $660 \mathrm{bp}$ in between the two fragments of TV239, a separate alignment was performed for each of these fragments. After the alignments were created in Clustal $X$, they were manually checked with BioEdit v $5.09\left[\mathrm{Hall}^{\odot}, 2001\right]$.

\subsubsection{Construction of phylogenetic trees}

Neighbor-Joining trees [Saitou and Nei, 1987] were drawn with MEGA $\vee 4.1$ [Tamura et al, 2007] of all the data. Three trees were drawn in total, one containing the NFLG fragments of R84, TV314 and TV412 with reference sequences, and two trees for the two fragments (gag-pol and env-nef) of 
TV239 also with reference sequences. The Kimura 2 parameter method of nucleotide substitution [Kimura, 1980] was used and a total of a 1000 bootstrap replicates was performed on each of the trees. The datasets were also analyzed with PAUP* version 4.0b10 (Phylogenetic Analysis using Parsimony* and other methods) [Swofford, 2002] for the construction of maximum likelihood trees.

The methods used by PAUP*, though far more thorough [Salemi and Vandamme, 2003], are extremely computationally intensive and the output format of the data needs far more additional work than with the use of other tree drawing software such as MEGA.

\subsection{Detection of recombinant viruses using RIP and Simplot}

Recombinant identification was performed with two widely used methods: Simplot [Lole et al, 1999] and the Recombinant Identification Program, RIP [Siepel et al, 1995]. The consensus alignment (excluding CRF01_AE) of RIP was used to query the sequences of interest. All NFLG fragments (R84, TV314 and TV 412) and the gag-pol and env-nef fragments of isolate TV239 were queried. The raw text files (.txt) of each of the fragments were uploaded into the program and a window size of $300 \mathrm{bp}$ was chosen.

The multiple alignments were also imported into Simplot version 3.5 [Lole et al, 1999; Salminen et al, 1995b] to identify recombination events within the NFLG samples and the two fragments of TV239. For the analysis of the NFLG fragments all three isolates (R84, TV314 and TV 412) were queried. A window size of $350 \mathrm{bp}$ and a step size of $50 \mathrm{bp}$ were selected. The gag-pol and envnef fragments of isolate TV239 were also queried with the use of the same window and step size as with the analysis of the NFLG fragments.

All sequences (R84, TV314 and TV 412) were first queried against all subtypes in the alignments for both the Simplot and the RIP analysis before each of the sequence analysis were rerun to simplify the output format. 
After the recombinant identification was completed Neighbor-Joining trees of the different recombinant sections were drawn. Reference sequences of pure subtype were obtained from the LANL database. Breakpoint coordinates which corresponded with the breakpoints that were obtained from the subtype and recombination identification done by the jpHMM analysis was used to obtain genome segments which corresponded with the same area of suspected viral recombination. Only a small number (10-15) of reference samples, which represented the most prominent viral subtypes were included in these datasets. After the various reference sequences were obtained multiple alignments of the different recombinant fragments were constructed with the use of Clustal X $v 1.81$ [Thompson $^{\odot}$ et al, 1997]. After the alignments were completed and was manually checked with BioEdit v 5.09 [Hall $\left.{ }^{\odot}, 2001\right]$ the different alignment files (.aln) were converted to MEGA format (.meg). These files were imported into MEGA v 4.1 [Tamura et al, 2007] to construct Neighbor-joining phylogenetic trees [Saitou et al, 1987]. The Kimura 2parameter [Kimura, 1980] was employed for the construction of the NJ-trees. A 100 bootstrap replicates were performed on all these datasets to confer statistical significance.

\subsection{Phylogenetic analysis of non-recombinant NFLG sequences}

The two isolates of a non-recombinant nature were also compared to other non-recombinant subtypes of $A$ and $B$. From the previous analysis it could be concluded that sample R84 was an HIV-1 subtype B virus and TV 314 a subtype $A 1$ virus. Subtype $B$ and $A 1$ sequences were obtained from the LANL database to compare with the sequences of interest. A BLAST was performed with R84 and TV314 and full-length sequences which were most closely related to the isolates were downloaded. Because R84 was collected in the mid 1980's more subtype B viruses from the 1980's and early 1990's were included in the subtype B dataset. After the full-length sequences were retrieved from the database, multiple alignments of the reference sequences with the sequences of interest were constructed with Clustal $X \quad v 1.81$ [Thompson $^{\odot}$ et al, 1997]. The isolate K.CM.96.MP535.AJ249239 was included for the use as an outgroup and all possible output formats were selected for 
each of the alignments. Each alignment were manually checked with BioEdit v 5.09 [Hall $\left.^{\odot}, 2001\right]$ after the alignments were done.

After the alignments were completed the alignment files (.aln) were converted to MEGA format (.meg). These files were then imported into MEGA $\vee 4.1$ [Tamura et al, 2007] and Neighbor-Joining phylogenetic trees [Saitou et al, 1987] were constructed with the use of the Kimura 2-parameter [Kimura, 1980]. Bootstrap analysis, with a total of a 1000 bootstrap replicates, were also performed on each of the datasets to infer statistical significance. 


\section{CHAPTER THREE - RESULTS}

\section{Table of Content}

Page

3.1 PCR amplification of partial gag, pol and env fragments

3.3 NJ phylogenetic tree analysis of partial gag, pol and env fragments

3.3.1 The gag $\mathrm{p} 24$ region

3.3.2 The pol-integrase region

3.3.3 The env gp41 region

3.4 Amplification and sequencing of NFLG of four samples

85

3.4.1 PCR amplification assays of $9.2 \mathrm{kbp}$ fragments of four samples

85

3.4.2 PCR amplification of four overlapping fragments 86

3.4.3 Sequences results of the NFLG fragments 86

3.5 Subtype identification of NFLG fragments with REGA and jpHMM online tools.

3.6 Construction of NFLG phylogenetic trees

3.7 Results of recombination identification with RIP and Simplot 92

3.7.1 Phylogenetic analysis of recombinant breakpoints 


\section{CHAPTER THREE - RESULTS}

\subsection{PCR amplification of partial gag, pol and env fragments}

The PCR results of the three subgenomic regions of the 12 samples are summarized in Table 3.1. Sample TV546 could not be amplified with the gag p24, pol-integrase and env gp41 amplification assays. TV546 was previously characterized as a subtype $G$ sample [Personal communication, Susan Engelbrecht] and thus may require the use of subtype specific primer sets. Other than TV 546 only the env gp41 amplification of sample TV 480 was unsuccessful. An example of the PCR results is illustrated in Figure 3.1

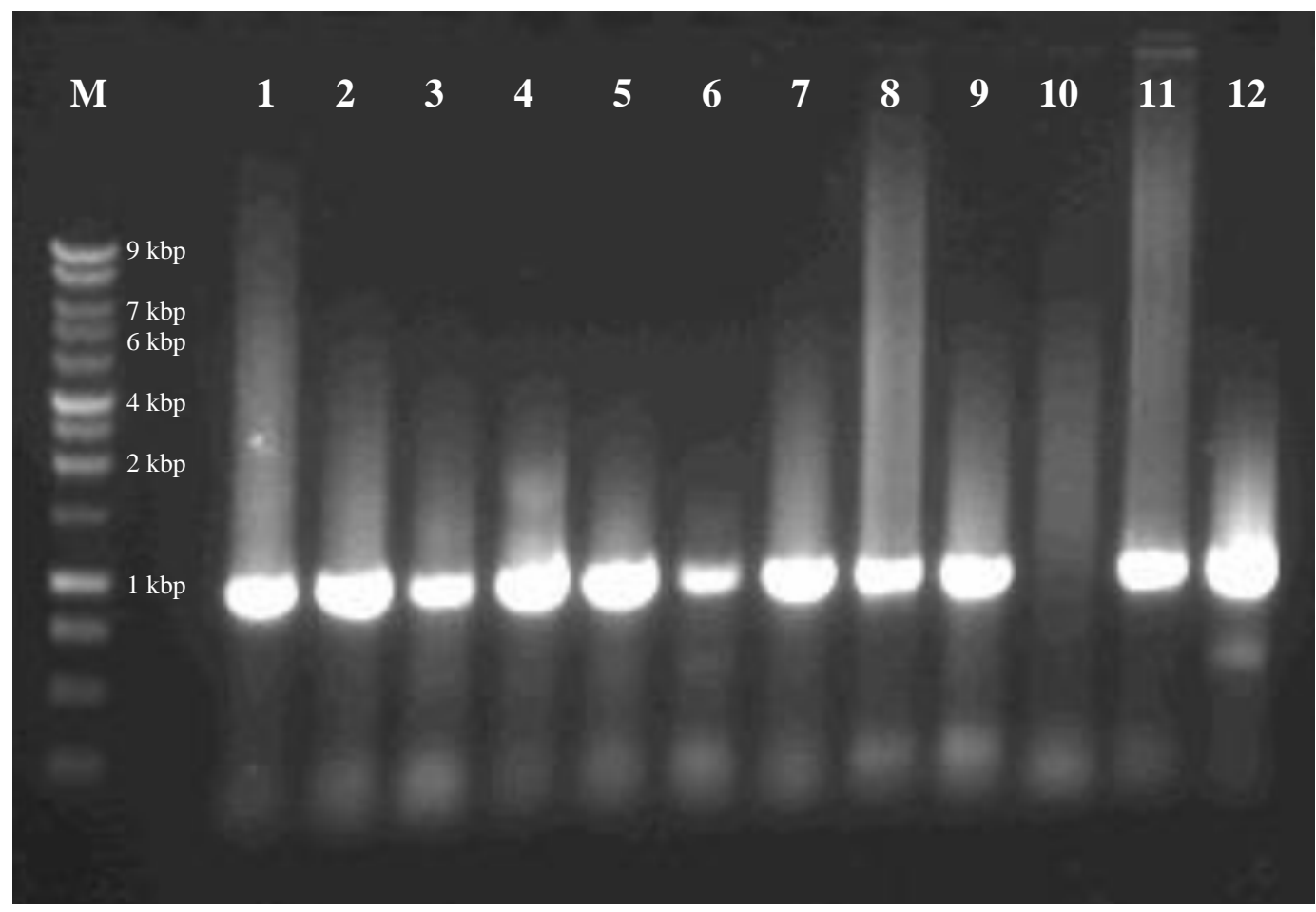

Figure 3.1: Agarose gel electrophoresis of the nested pol-integrase PCR products. Lanes: Lane M - 1kb marker, Lane 1 - TV412, Lane 2 - TV314, Lane 3 - TV340, Lane 4 - TV218, Lane 5 - TV239, Lane 6 - TV 101, Lane 7 - TV480, Lane 8 - TV 86, Lane 9 - TV515, Lane 10 - TV546, Lane 11 - TV 412, and Lane 12 - R84. 
Table 3.1 PCR amplification of the subgenomic regions of the 12 samples.

\begin{tabular}{|c|c|c|c|}
\hline \multicolumn{4}{|c|}{ PCR Results } \\
\hline Sample & gag p24 & pol-integrase & env gp41 \\
\hline R84 & Positive & Positive & Positive \\
\hline TV 86 & Positive & Positive & Positive \\
\hline TV 101 & Positive & Positive & Positive \\
\hline TV 218 & Positive & Positive & Positive \\
\hline TV 239 & Positive & Positive & Positive \\
\hline TV 314 & Positive & Positive & Positive \\
\hline TV 340 & Positive & Positive & Positive \\
\hline TV 412 & Positive & Positive & Positive \\
\hline TV 441 & Positive & Positive & Positive \\
\hline TV 480 & Positive & Positive & Negative \\
\hline TV 515 & Positive & Positive & Positive \\
\hline TV 546 & Negative & Negative & Negative \\
\hline
\end{tabular}

The sequencing of positive PCR products was successful with one exception. The gag p24 sequence of TV340 could not be used due to multiple peaks in the electropherogram. The sequencing results of the three subgenomic fragments are summarized in Table 3.2. The text files of the sequences are presented in Appendix A. The sequences were also submitted to Genbank with accession numbers FJ647150 - FJ647168.

Table 3.2: Sequencing results for the subgenomic regions.

\begin{tabular}{|c|c|c|c|}
\hline \multicolumn{4}{|c|}{ Sequencing Results } \\
\hline Sample & gag p24 & pol-integrase & env gp41 \\
\hline R84 & Positive & Positive & Positive \\
\hline TV 86 & Positive & Positive & Positive \\
\hline TV 101 & Previous data* & Previous data* & Previous data* \\
\hline TV 218 & Previous data* $^{*}$ & Previous data* & Previous data* $^{*}$ \\
\hline TV 239 & Positive & Positive & Positive \\
\hline TV 314 & Positive & Positive & Positive \\
\hline TV 340 & Negative & Positive & Positive \\
\hline TV 412 & Positive & Positive & Positive \\
\hline TV 441 & Positive & Positive & Positive \\
\hline TV 480 & Positive & Positive & Negative \\
\hline TV 515 & Positive & Positive & Positive \\
\hline
\end{tabular}

Key: * Previous data (Personal communication, Susan Engelbrecht)

\subsection{Subtyping of the partial gag, pol and env regions}

The results of the viral subtyping done with the REGA and jpHMM viral subtyping tools as well as the NJ-trees of the three genomic fragments are 
presented in sections 3.3.1 (gag p24), 3.3.2 (pol integrase) and 3.3.3 (env gp41) respectively. The phylogenetic data and subtyping results are summarized in Table 3.3.

The results of the online viral subtyping will be presented in Appendix $B$ Figures 6.1- 6.3 and Tables 6.1 - 6.3, illustrates the REGA and jpHMM results respectively.

\subsection{NJ phylogenetic tree analysis of partial gag, pol and env fragments.}

The results of the NJ phylogenetic analysis for the gag, pol and env fragments will be discussed in the following sections $(3.3 .1-3.3 .3)$.

\subsubsection{The gag p24 region}

For the NJ-tree of the 10 gag p24 sequences (Figure 3.2) with the reference sequences from the LANL database [http://www.hiv.lanl.gov/], four samples (TV314, TV412, TV239, and TV101) clustered with subtype A1 sequences. One sample, TV515, clustered with subtype F1 sequences in the tree. Sample R84, clustered with other subtype B strains in the tree. The other four samples (TV86, TV218, TV441, and TV480) clustered amongst other subtype C isolates in the tree. All these subtypes were confirmed with high bootstrap values. The clustering pattern of the gag sequences of the 10 samples in the $\mathrm{NJ}$-tree corresponded with the subtyping results that were obtained with the REGA and jpHMM analysis. 
Table 3.3: Subtyping analysis performed on the gag p24, pol-integrase and env gp41 fragments.

\begin{tabular}{|c|c|c|c|c|c|c|c|c|c|c|}
\hline \multirow[b]{2}{*}{ Sample } & \multicolumn{3}{|c|}{ gag p24 } & \multicolumn{3}{|c|}{ pol-integrase } & \multicolumn{3}{|c|}{ env gp41 } & \multirow[b]{2}{*}{$\begin{array}{l}\text { Assumed } \\
\text { subtype }\end{array}$} \\
\hline & $\begin{array}{l}\text { jpHMM viral } \\
\text { subtyping }\end{array}$ & $\begin{array}{l}\text { REGA viral } \\
\text { subtyping }\end{array}$ & $\begin{array}{l}\text { Clustering } \\
\text { pattern in } \\
\text { NJ-tree }\end{array}$ & $\begin{array}{l}\text { jpHMM viral } \\
\text { subtyping }\end{array}$ & $\begin{array}{l}\text { REGA viral } \\
\text { subtyping }\end{array}$ & $\begin{array}{l}\text { Clustering } \\
\text { pattern in } \\
\text { NJ-tree }\end{array}$ & $\begin{array}{l}\text { jpHMM viral } \\
\text { subtyping }\end{array}$ & $\begin{array}{l}\text { REGA viral } \\
\text { subtyping }\end{array}$ & $\begin{array}{l}\text { Clustering } \\
\text { pattern in } \\
\text { NJ-tree }\end{array}$ & \\
\hline R 84 & B & B & B & B & B & B & $B$ & B & B & B \\
\hline TV86 & C & $\mathrm{C}$ & $\mathrm{C}$ & C & $\mathrm{C}$ & $\mathrm{C}$ & C & $\mathrm{C}$ & $\mathrm{C}$ & C \\
\hline TV101 & A1 & $\mathrm{A} 1$ & $\mathrm{~A} 1$ & A1D & $\mathrm{A} 1^{*}$ & Unclass & $\mathrm{A} 1$ & $\mathrm{~A} 1$ & $\mathrm{~A} 1$ & $\begin{array}{c}\mathrm{A} 1 \\
\text { Recombinant }\end{array}$ \\
\hline TV218 & C & C & C & C & C & C & A1 & $\mathrm{A} 1^{*}$ & $\mathrm{~A} 2$ & $\begin{array}{c}\text { CA } \\
\text { Recombinant }\end{array}$ \\
\hline TV239 & A1 & $\mathrm{A} 1$ & $\mathrm{~A} 1$ & $\mathrm{~A} 1 \mathrm{H}$ & $\mathrm{A} 1^{*}$ & $\mathrm{~A} 1$ & $\mathrm{~A} 1$ & $\mathrm{~A} 1^{*}$ & A1 / A2 & $\begin{array}{c}\mathrm{A} 1 \\
\text { Recombinant }\end{array}$ \\
\hline TV314 & A1 & $\mathrm{A} 1$ & $\mathrm{~A} 1$ & A1J & $\mathrm{A} 1^{*}$ & $\mathrm{~A} 1$ & $\mathrm{~A} 1$ & $\mathrm{~A} 1^{*}$ & A1 / A2 & A1 \\
\hline TV340 & - & - & - & GB & $\mathrm{G}^{*}$ & G & A1 & $\mathrm{A} 1$ & $\mathrm{~A} 1$ & $\begin{array}{c}\text { ABG } \\
\text { Recombinant }\end{array}$ \\
\hline TV412 & A1 & $\mathrm{A} 1$ & $\mathrm{~A} 1$ & A1DJ & $\mathrm{A} 1^{*}$ & $\mathrm{~A} 1$ & $\mathrm{~A} 1$ & $\mathrm{~A} 1$ & $\mathrm{~A} 1$ & $\begin{array}{c}\mathrm{A} 1 \\
\text { Recombinant }\end{array}$ \\
\hline TV441 & C & C & C & C & $C^{*}$ & C & $\mathrm{A} 1$ & $\mathrm{~A} 1^{*}$ & $\mathrm{~A} 2$ & $\begin{array}{c}\text { CA } \\
\text { Recombinant }\end{array}$ \\
\hline TV480 & C & C & C & CJ & $C^{*}$ & C & - & - & - & $\begin{array}{c}C \\
\text { Recombinant }\end{array}$ \\
\hline TV515 & $\mathrm{F} 1$ & F1 & F1 & $\mathrm{F} 1$ & F1 & $\mathrm{F} 1$ & F1 & F1 & F1 & F1 \\
\hline
\end{tabular}

Key: * (the bootstrap values of the analysis was not supportive or < 70\%), multi state characters e.g. A1D (indicates possible recombination events), 1

(sample clustered with both the subtypes or sub-subtypes), Unclass (Unclassified), and - (PCR or sequencing reactions were not successful) 


\subsubsection{The pol-integrase region}

In the pol-integrase tree (Figure 3.3), TV515 and R84, clustered with other F1 and B sequences respectively. Three samples (TV412, TV314, and TV239) clustered with other $A 1$ isolates. These three samples were identified as $A 1$ recombinants with the REGA and jpHMM subtyping and recombination analysis (Table 3.2). TV101 did not cluster with any of the 9 subtypes in the tree, which indicates possible viral recombination in this sequence. jpHMM analysis indicated that TV101 might be an AD recombinant within the polintegrase sequenced fragment. Sample TV441 was an outlier of the C cluster, but with the online subtyping results was continuously identified as a subtype $C$ isolate. TV340 was an outlier of the subtype $G$ cluster which might indicate possible recombination. The REGA subtyping tool identified TV340 as an subtype $G$ sample but with a very low bootstrap support. jpHMM analysis of TV340 however identified the sample as AG recombinant form.

\subsubsection{The env gp41 region}

In the env NJ-tree three (Figure 3.4 ) the following samples clustered with other A1 samples: TV101, TV340 and TV412 which corresponds well with the subtyping results of the REGA and jpHMM analysis. TV218 and TV441 clustered with other A2 sequences, with TV239 and TV314 outliers of the A1/A2 cluster, indicating possible recombination. REGA and jpHMM analysis of these four samples revealed that all these samples (TV218, TV239, TV314 and TV412) were subtype A1 in the env gp41 region. As with the gag and pol trees TV86, TV515, and R84 clustered with other C, F1, and B sequences respectively and corresponded well with the online subtyping results. 


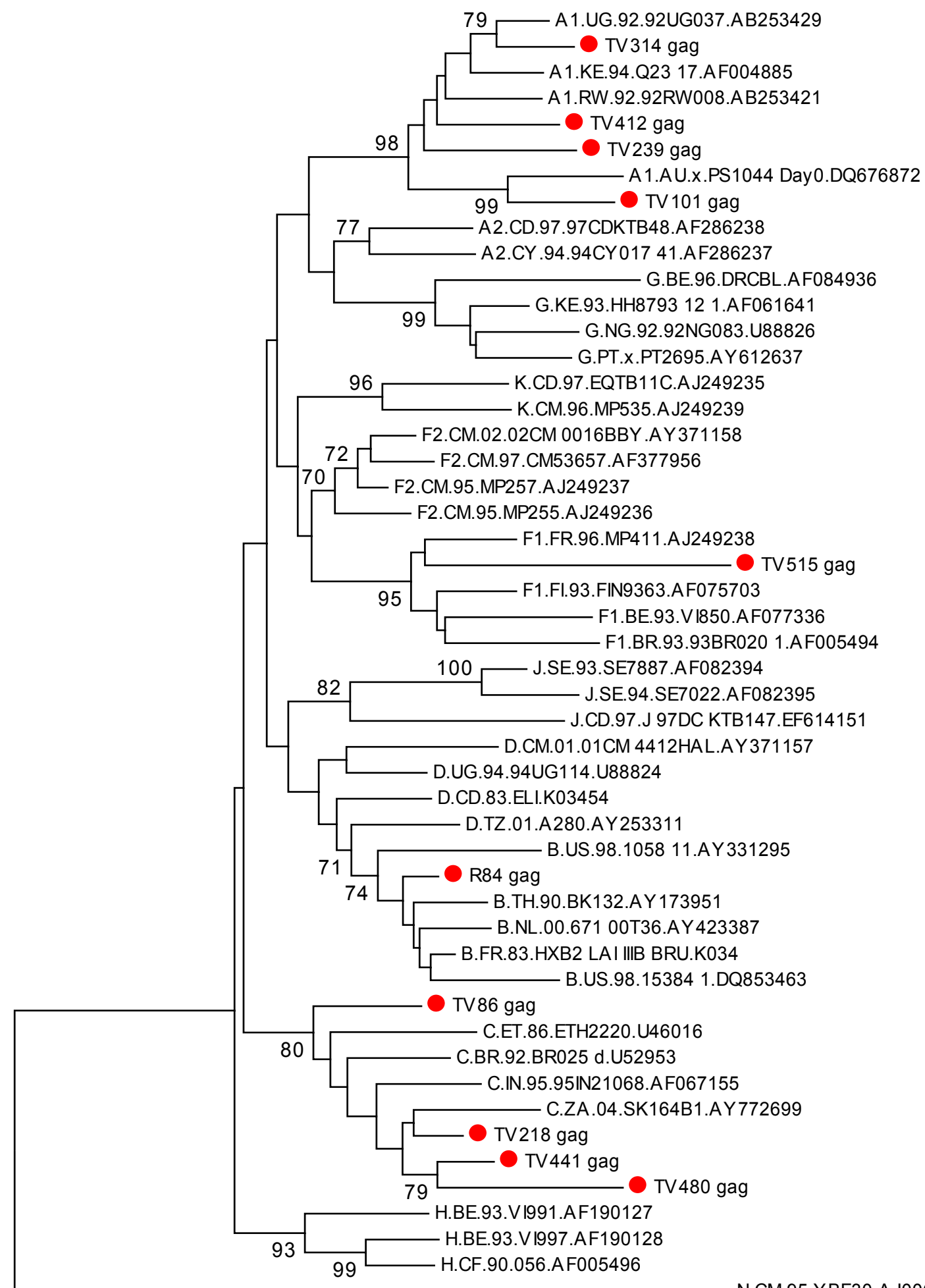

N.CM.95.YBF30.AJ006022

0.02

Figure 3.2: A Neighbor-joining tree of gag sequences (485 bp) indicating reference sequences and TV sequences. The TV sequences are indicated with a red dot. In the bottom line the genetic distance, which corresponds to the length of the branches, is shown. Only bootstrap values greater than 70 percent are included. 


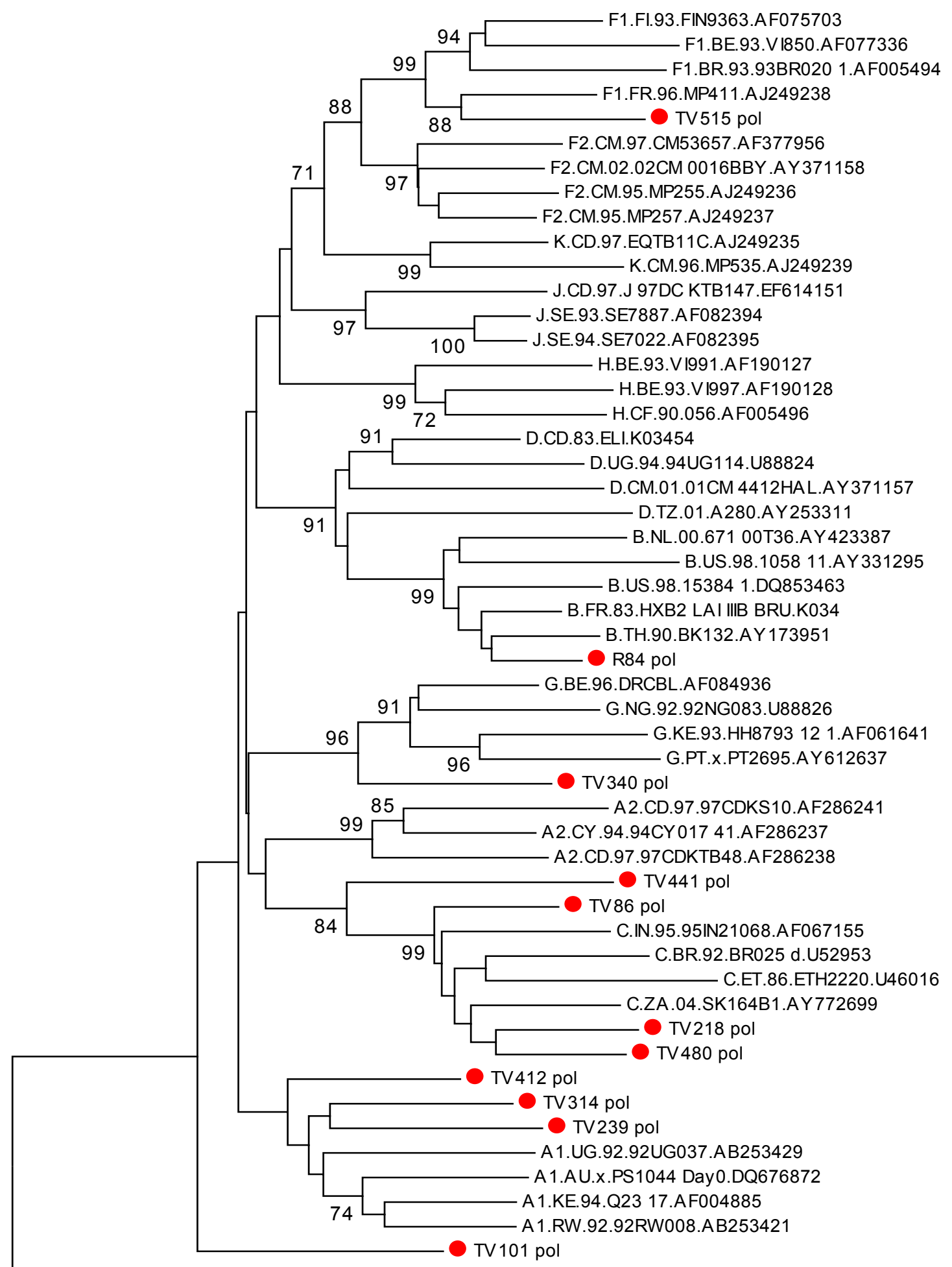

N.CM.95.YBF30.AJ006022

Figure 3.3: A Neighbor-joining tree of pol sequences (944 bp) indicating reference sequences and TV sequences. Each TV sequences are indicated with a red dot. The genetic distance, which corresponds to the length of the branches, is shown in the bottom line. Only bootstrap values greater than 70 percent are included. 


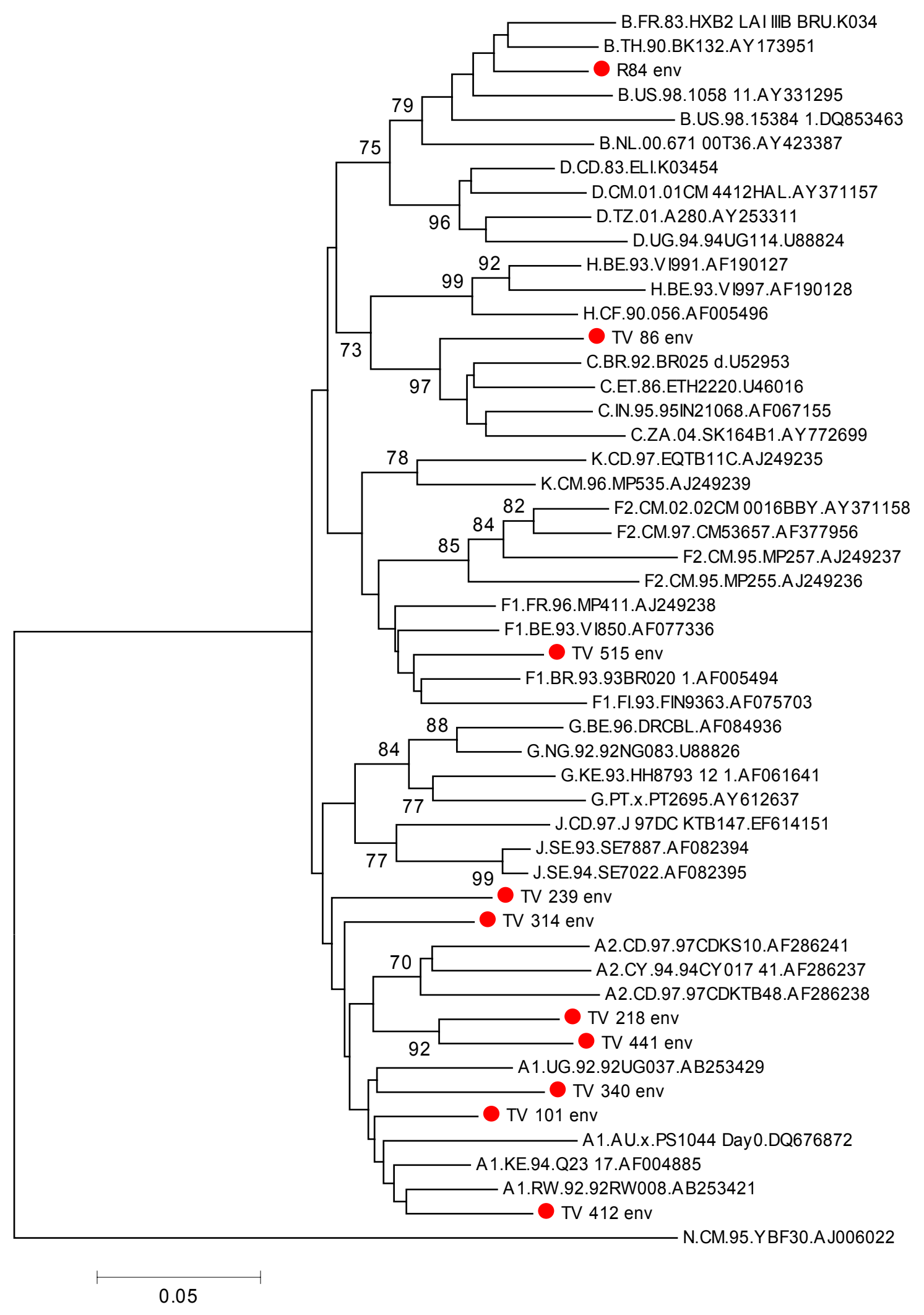

Figure 3.4: A Neighbor-joining tree of env sequences (438 bp) indicating reference sequences and TV sequences. Each TV sequences are indicated with a red dot. The genetic distance, which corresponds to the length of the branches, is shown in the bottom line. Only bootstrap values greater than 70 percent are included. 


\subsection{Amplification and sequencing of NFLG of four samples}

\subsubsection{PCR amplification assays of $9.2 \mathrm{kbp}$ fragments of four samples}

The long amplification assays (9.2 kbp) of the four samples (R84, TV239, TV314, and TV412) were not successful. Due to limited template DNA, a large amount of $9.2 \mathrm{kbp}$ PCR products could not be obtained for sequencing. An example of the results of long PCR is shown in Figure 3.5.

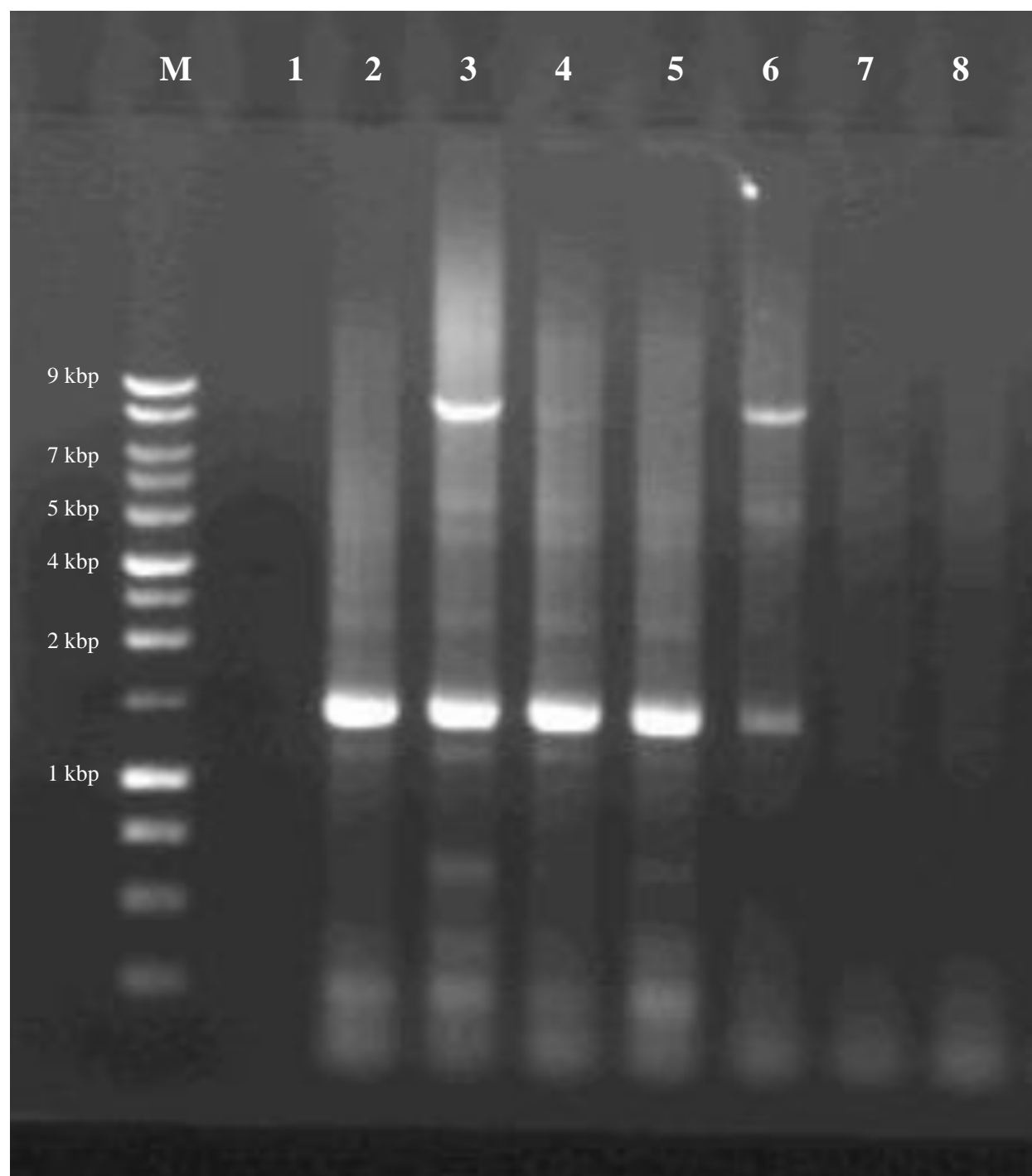

Figure 3.5: Agarose gel of the unsuccessful $9.2 \mathrm{kbp}$ PCR products of sample

R84. Lanes: lane M - $1 \mathrm{kbp}$ Molecular Marker, lane 1 - Blank, lane $2-500 \mathrm{ng} / \mathrm{\mu l}$ of template DNA, lane $3-400 \mathrm{ng} / \mu \mathrm{l}$ of template DNA, lane $4-300 \mathrm{ng} / \mu \mathrm{l}$ of template DNA, lane 5 - 200 $\mathrm{ng} / \mu \mathrm{l}$ of template DNA, lane $6-100 \mathrm{ng} / \mu \mathrm{l}$ of template DNA, lanes $7 \& 8-$ Negative Control 


\subsubsection{PCR amplification of four overlapping fragments}

Sample R84 were amplifiable for all four fragments (LTR-gag, gag-pol, polenv, and env-LTR). For the other three samples (TV239, TV314, and TV412), only the amplification of the gag-pol, pol-env, and env-LTR fragments were successful (data not shown). The results of the PCR amplification assays are summarized in Table 3.4 .

Table 3.4: PCR amplification of the four overlapping fragments.

\begin{tabular}{|c|c|c|c|c|}
\hline \multirow{2}{*}{ Sample } & \multicolumn{4}{|c|}{ Fragment } \\
\cline { 2 - 5 } & LTR-gag & gag-pol & pol-env & env-LTR \\
\hline R 84 & Positive & Positive & Positive & Positive \\
\hline TV239 & Negative & Positive & Positive & Positive \\
\hline TV314 & Negative & Positive & Positive & Positive \\
\hline TV412 & Negative & Positive & Positive & Positive \\
\hline
\end{tabular}

\subsubsection{Sequences results of the NFLG fragments}

A continues fragment of sample R84, stretching from the start of the gag region up to the 3'LTR region (position 601-9514 relative to HXB2) were obtain from the sequenced data. The sequencing of the other samples was obtained with mixed results. All attempts to sequence the 5'LTR-gag regions of the other three isolates (TV239, TV314, and TV412) as well as the $660 \mathrm{bp}$ gap between the two fragments of sample TV239 from the $9.2 \mathrm{kbp}$ PCR fragment were unsuccessful. This resulted in; two fragments for TV 239 stretching from position 1245 - 5534 and 6195 - 9146 (relative to HXB2 coordinates), a single continues fragment for TV314 stretching from 1235 9551 and a single fragment, stretching from position 1246 - 8254, for sample TV412.

Each sequence fragment was analyzed with the Gene Cutter tool from LANL database. No pre-mature stop codons could be found in any of the open reading frames of the various genes. The results of the Gene Cutter analysis for each of the four isolates sequences are presented in Appendix D. All the near full-length sequences were submitted to GenBank with accession numbers FJ647145 - FJ647149. 


\subsection{Subtype identification of NFLG fragments with REGA and jpHMM online tools.}

The online subtyping and recombination results, which was performed with the jpHMM and REGA viral subtyping tools, of the three near full-length sequences as well as the two fragments of sample TV239 are summarized in Table 3.5. For the full results of the analysis please refer to Appendix $E$, Figure 6.4 and Table 6.8, for the REGA and jpHMM results respectively.

Table 3.5: jpHMM and REGA subtyping tools.

\begin{tabular}{|l|c|c|}
\hline \multicolumn{1}{|c|}{ Sample } & jpHMM subtyping tool & REGA subtyping tool \\
\hline R 84 & $\mathrm{B}$ & $\mathrm{B}^{*}$ \\
\hline TV 239 gag-pol & $\mathrm{A} 1$ & $\mathrm{~A} 1^{*}$ \\
\hline TV 239 env-nef & $\mathrm{C} / \mathrm{A} 1 / \mathrm{C} / \mathrm{A} 1 / \mathrm{C}$ & $\mathrm{C} / \mathrm{A} 1 / \mathrm{C}{ }^{*}$ \\
\hline TV314 & $\mathrm{A} 1$ & $\mathrm{~A} 1^{*}$ \\
\hline TV 412 & $\mathrm{A} 1 / \mathrm{D} / \mathrm{A} 1 / \mathrm{D} / \mathrm{A} 1$ & $\mathrm{~A} 1 / \mathrm{D} / \mathrm{A} 1^{*}$ \\
\hline
\end{tabular}

Key: * (The REGA reports of each of the analysis were checked and all subtyping and recombination patterns are with bootstrap support - >70 percent), and / (indicate possible recombination events).

From the analysis of the four isolates with the two different subtyping and recombination tools one can conclude that: R84 is subtype $B$ isolate, TV239 is a $A C$ recombinant virus (with the gag-pol fragment belonging to subtype $A 1$ and the env-nef fragment showing signs of $A C$ recombination breaking repeatedly throughout the fragment). Similarly, TV314 was classified as a subtype A1 isolate and TV412 is an AD recombinant virus (also breaking repeatedly throughout the fragment) by both the subtyping methods.

Though both the REGA and the jpHMM analysis were able to correctly identify the four isolates, the jpHMM subtyping tool was found to be much more accurate due to the phylogenetic approach used by the program. 


\subsection{Construction of NFLG phylogenetic trees}

Three NJ-trees (one containing the three sequences of the NFLG fragments and two for each of the TV239 fragments) were constructed with the use of $M E G A \vee 4.1$. Data sets were also analyzed with PAUP $\vee 4.0 \mathrm{~b} 10$ and PhyML $\vee 3.0$ and maximum likelihood trees were constructed with the use of these programs as well as the HKY and GTR models of nucleotide substitution (data not shown).

Three NJ-trees were constructed with the use of MEGA $\vee$ 4.1. The NeighborJoining tree of the three NFLG's containing samples R84, TV314 and TV412 as well as several reference strains from the LANL database can be seen in Figure 3.6. In the tree (Figure 3.6) two sample TV314 and R84 cluster within subtype clusters. R84 clustered with other subtype $B$ viruses and was most closely related to B.FR.83.HXB2 LAI IIIB BRU.KO34 with a strong bootstrap support of $94 \%$. TV314 clustered with A1 sequences and was more closely related to the reference strain A1.UG .92 .92UG037. AB253429, than to any of the other samples in the cluster, but with a very low bootstrap support of only $42 \%$. Sample TV412 was an outlier of the A1 cluster which possibly indicates viral recombination between subtype A1 and another HIV-1 subtype. The outgroup, N. CM . 95 . YBF30 . AJ๑06022, rooted the tree.

Figures 3.7 and 3.8 shows the NJ-trees of sample TV239's two fragments (gag-pol and env-nef) respectively. 


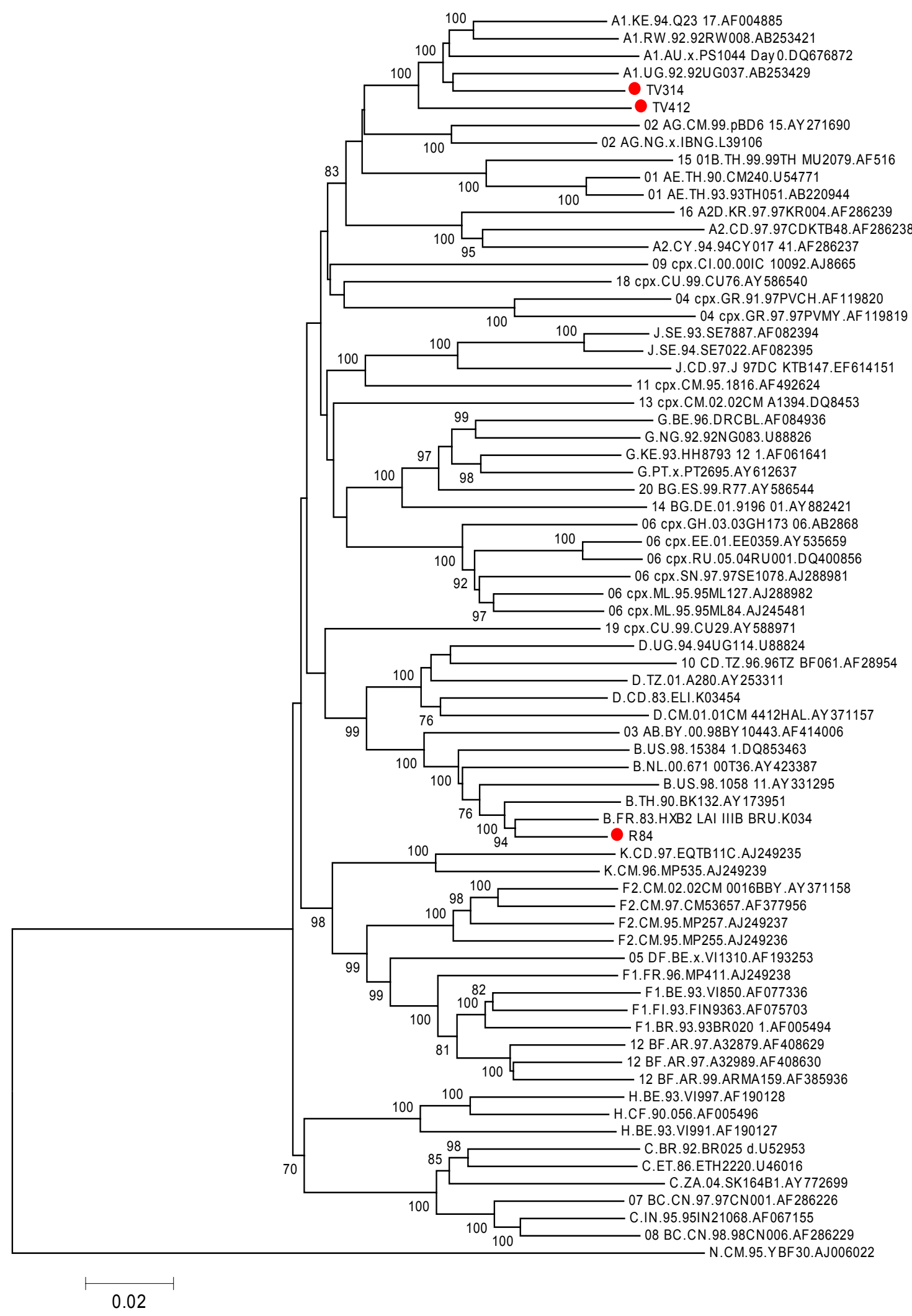

Figure 3.6: A Neighbor-joining tree containing the NFLG sequences of the three TV samples and reference samples. Each TV sequences are indicated with a red dot. The genetic distance is shown in the bottom line and corresponds to the length of the branches. Bootstrap values greater than 70 percent are shown. 


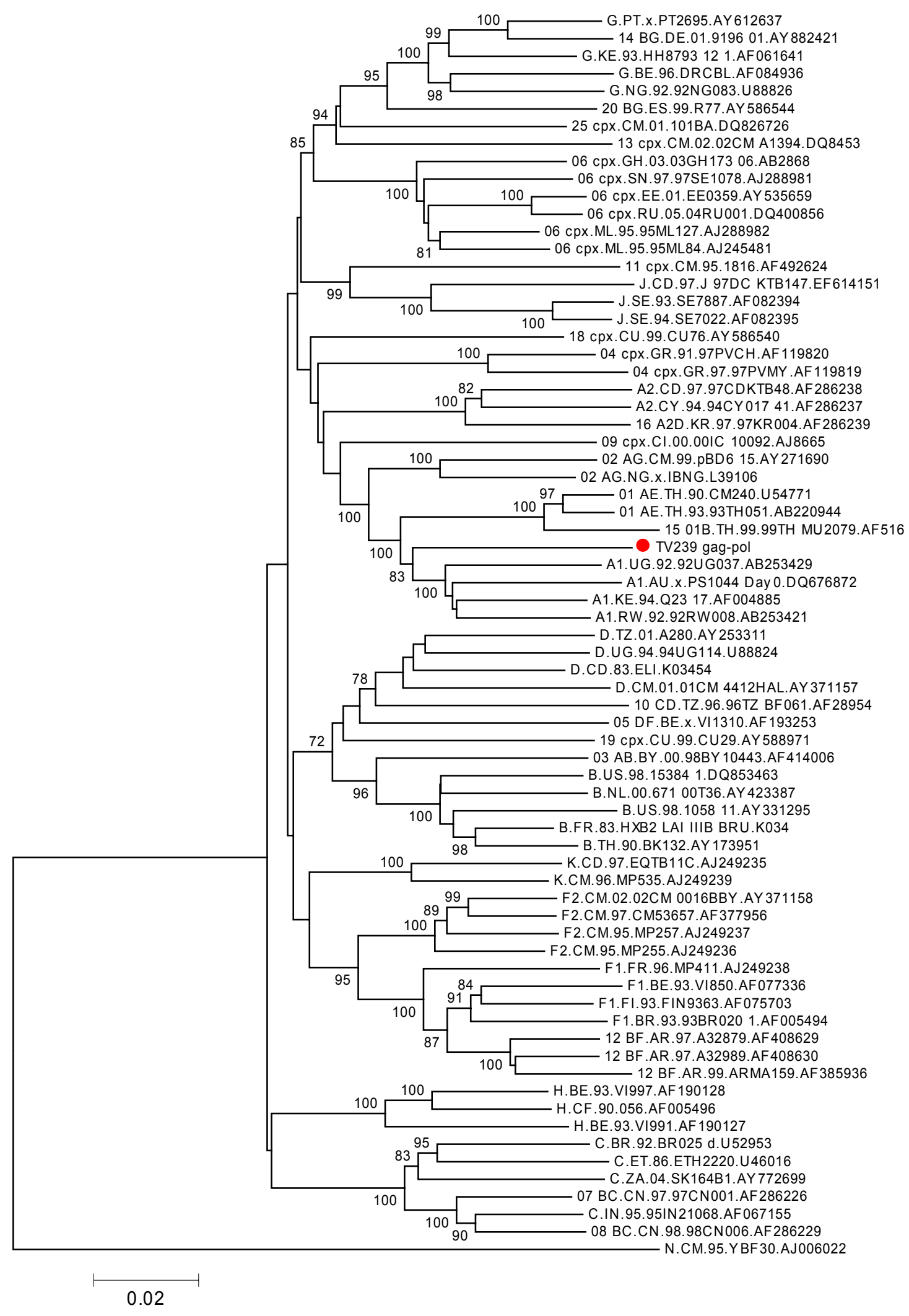

Figure 3.7: A Neighbor-joining tree containing reference sequences and the sequence of the gag-pol fragment of TV239. The TV sequence is marked with a red dot. The genetic distance, which corresponds to the branch lengths, is shown at the bottom. Bootstrap values greater than 70 percent are shown. 


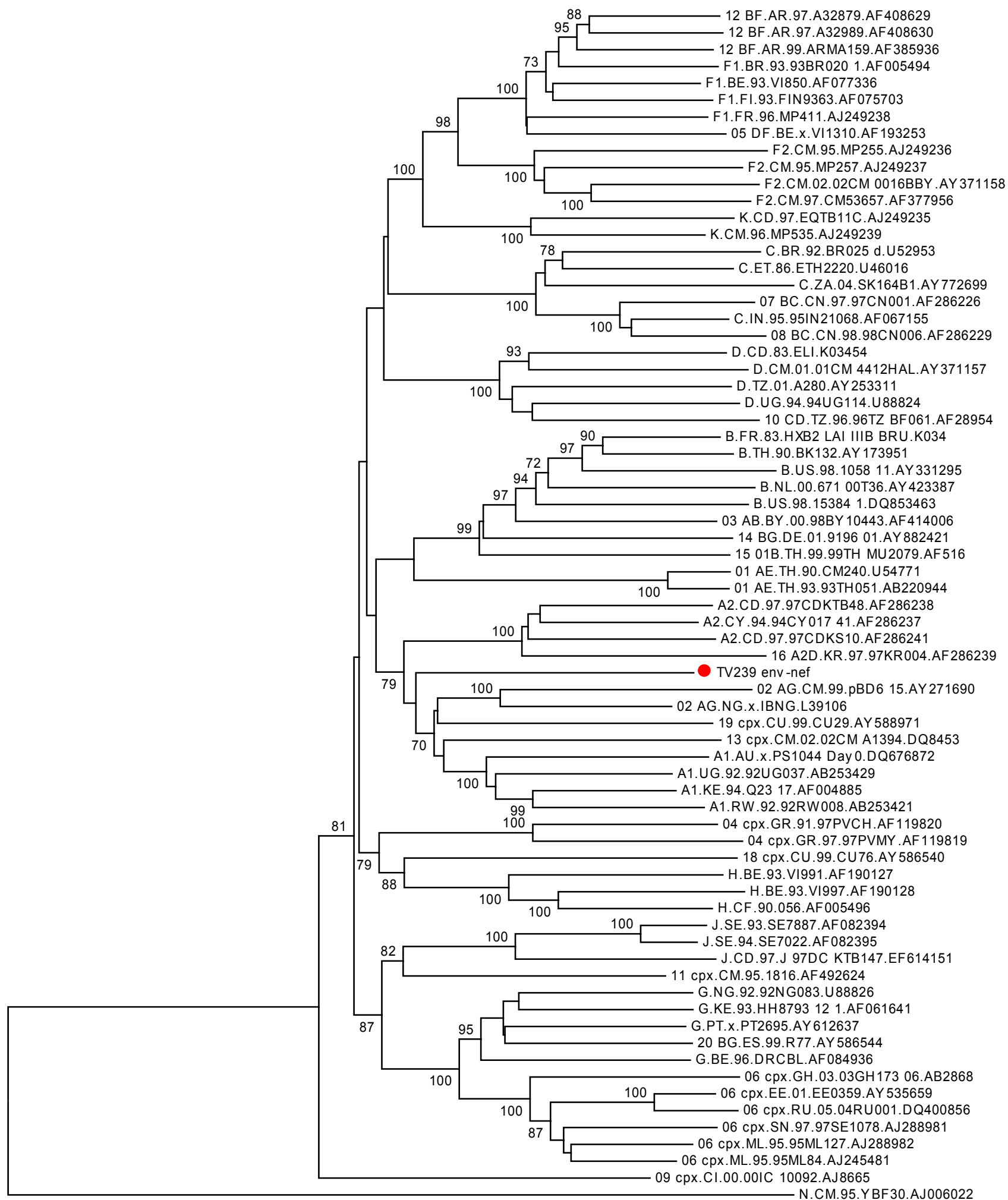

0.05

Figure 3.8: A Neighbor-joining tree containing reference sequences and the sequence of the env-nef fragment of TV239. The TV sequence is marked with a red dot. The genetic distance, which corresponds to the branch lengths, is shown at the bottom. Bootstrap values greater than 70 percent are shown. 
Inspection of these trees revealed that out of the 71 taxa in the trees: the gagpol fragment clearly clustered with other subtype A1 isolates in the tree with a strong bootstrap support of $83 \%$ and that the env-nef fragment was an outlier with a bootstrap support of $53 \%$, containing $A 1$ isolates as well as CRF02, cpx 19 and cpx 13 reference strains. CRF13_cpx is a complex recombinant form containing viral segments from subtypes $A, G$, and $J$ as well as fragments of CRF01_AE. The CRF19_cpx sequence on the other hand contains viral segments from subtypes $A 1, D$ and $G$. From the analysis of the two fragments of sample TV239 it is clear that this isolate can be considered as an A1 recombinant virus, with the gag-pol fragment as a subtype A1 and the env-nef fragment as the breakpoint of viral recombination.

\subsection{Results of recombination identification with RIP and Simplot}

The analysis of the four samples revealed recombination within two of the isolates genomes. TV412 and TV239 were identified by both the REGA and jpHMM subtype and recombination identification programs as an $A D$ and $A C$ recombinant respectively.

Apart from the REGA and jpHMM analysis the near-full length sequences were also analyzed with the use of two widely used recombination identification programs to observe potential recombination events: RIP [Siepel et al, 1995] and Simplot. For the RIP analysis, the consensus alignment of the LANL database [http://www.hiv.lanl.gov/] was used with a window size of 300 bp to obtain the results.

Only two of the four isolates (TV239 and TV412) showed signs of viral recombination. Similarity plots for these two isolates were downloaded and are presented in Figures 3.9 and 3.10. The similarity plot analysis in RIP of samples R84 and TV314 indicated that: R84 had a high similarity with other subtype B viral subtypes and that TV314 had a high similarity with other subtype A1 isolates in the LANL reference alignment. Similarly the analysis of the gag-pol fragment of TV239 also showed a high similarity with other A1 subtypes in the LANL reference alignment. Only the env-nef fragment of 
sample TV239 (Figure 3.10) and the fragment of sample TV412 (Figure 3.11) showed signs of viral recombination.

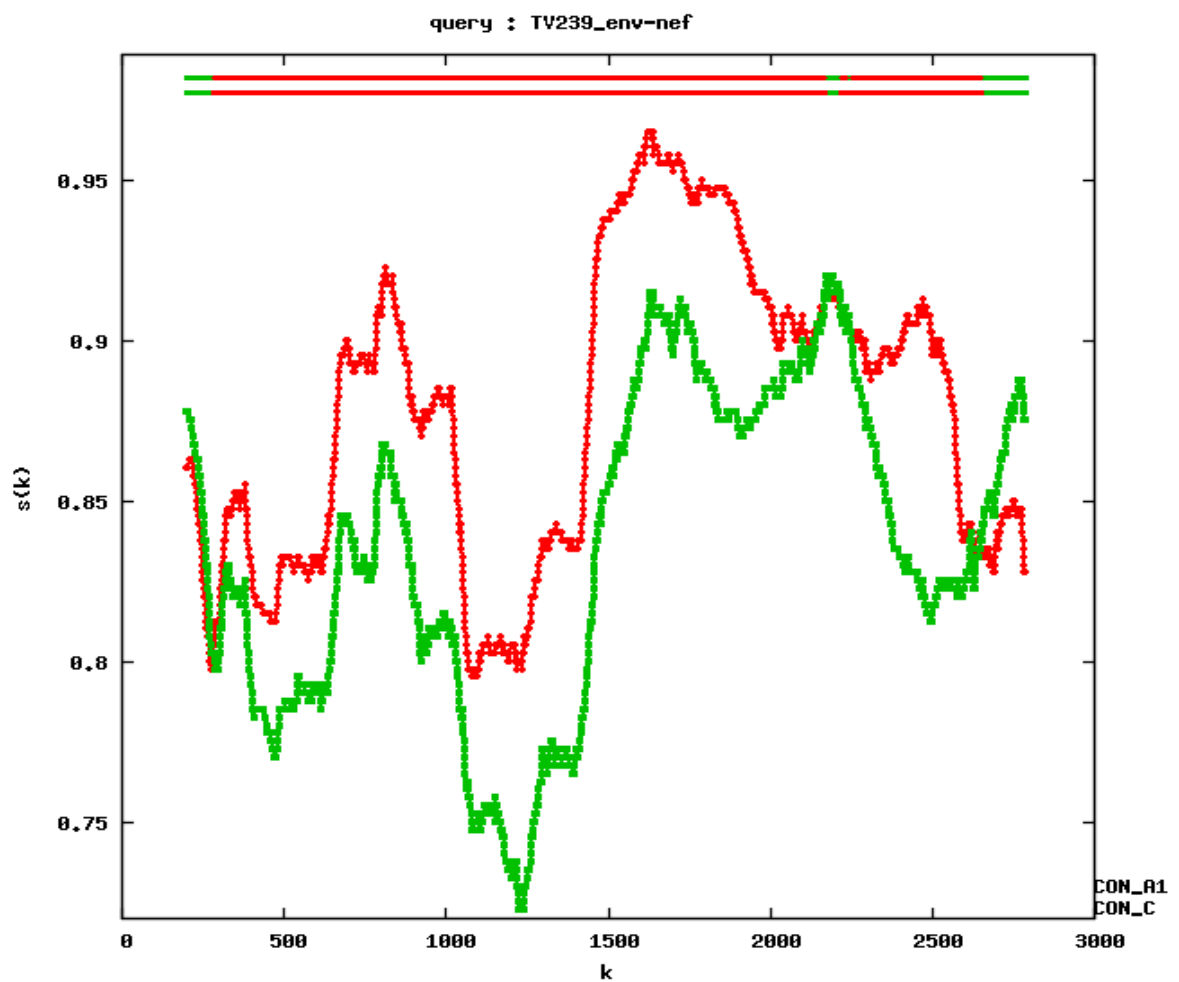

Figure 3.9: The analysis of the TV239 env-nef fragment with RIP. The s and the $\mathrm{k}$ symbols on the vertical and horizontal axis represent similarity and distance (in nucleotides or base pairs) respectively.

The RIP similarity analysis of the env-nef fragment of TV239 indicates AC recombination within the fragment, with multiple breakpoints. The analysis of TV412 also indicated recombination within the fragment with the genome breaking between subtype $A$ and $D$ viruses throughout the fragment. The results of the RIP analysis roughly corresponds to the breakpoints and recombination pattern that was obtained from the jpHMM analysis. 


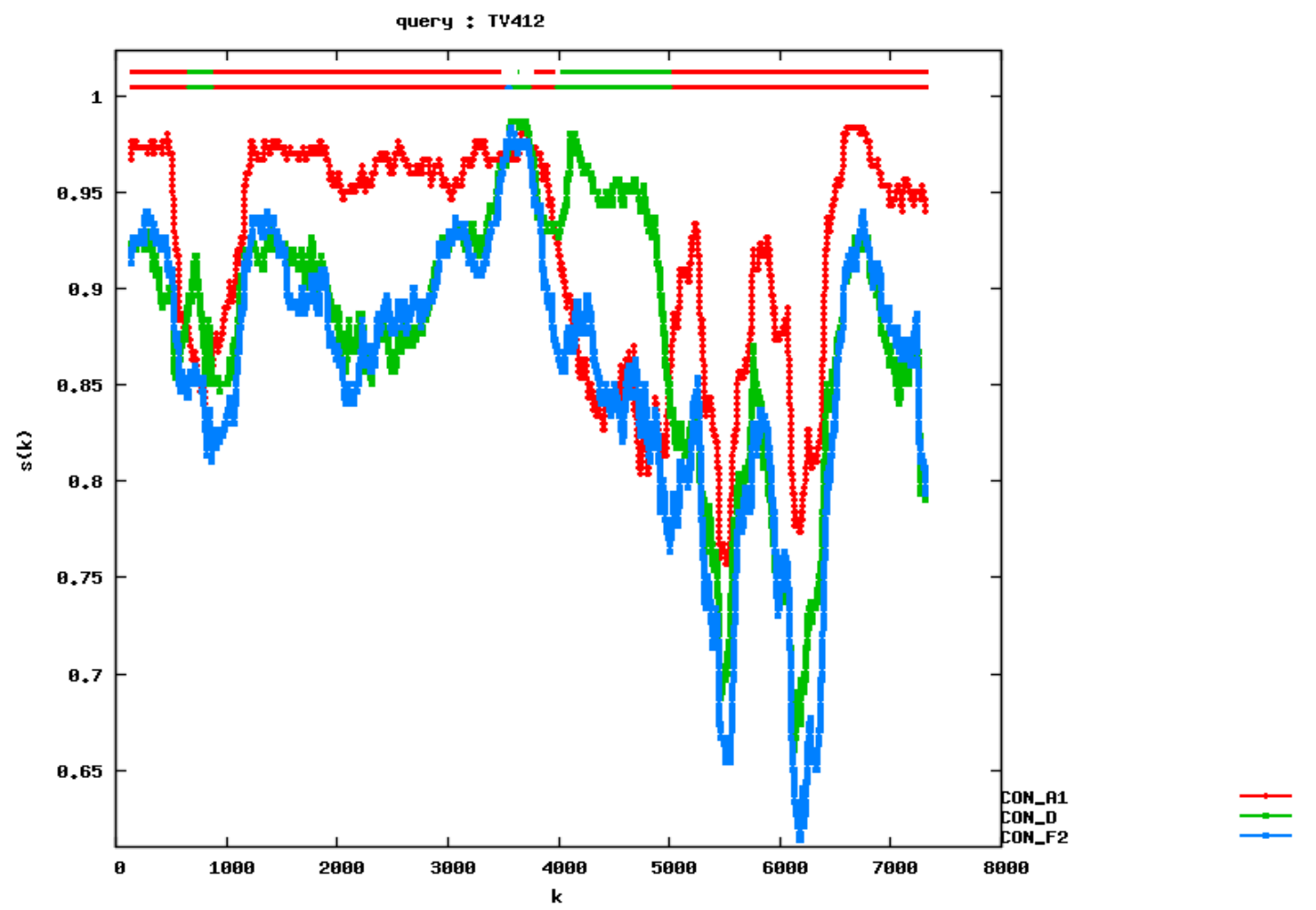

Figure 3.10: The analysis of TV412 with RIP. The $s$ and the $k$ symbols on the vertical and horizontal axis represent similarity and distance (in nucleotides or base pairs) respectively.

The four samples were also analyzed with Simplot v 3.5 [Lole et al, 1999; Salminen et al, 1995]. As with the RIP analysis samples R84, TV314 did not show signs of viral recombination and had high similarities with subtype $B$ and A1 isolates respectively. The analysis of the TV239 fragments showed that the gag-pol fragment was a subtype $A 1$ isolate whereas the env-nef (Figure 3.11) fragment showed signs of AC recombination. The analysis of TV412 (Figure 3.12) indicated viral recombination, between subtypes $A$ and $D$, within the fragment. 


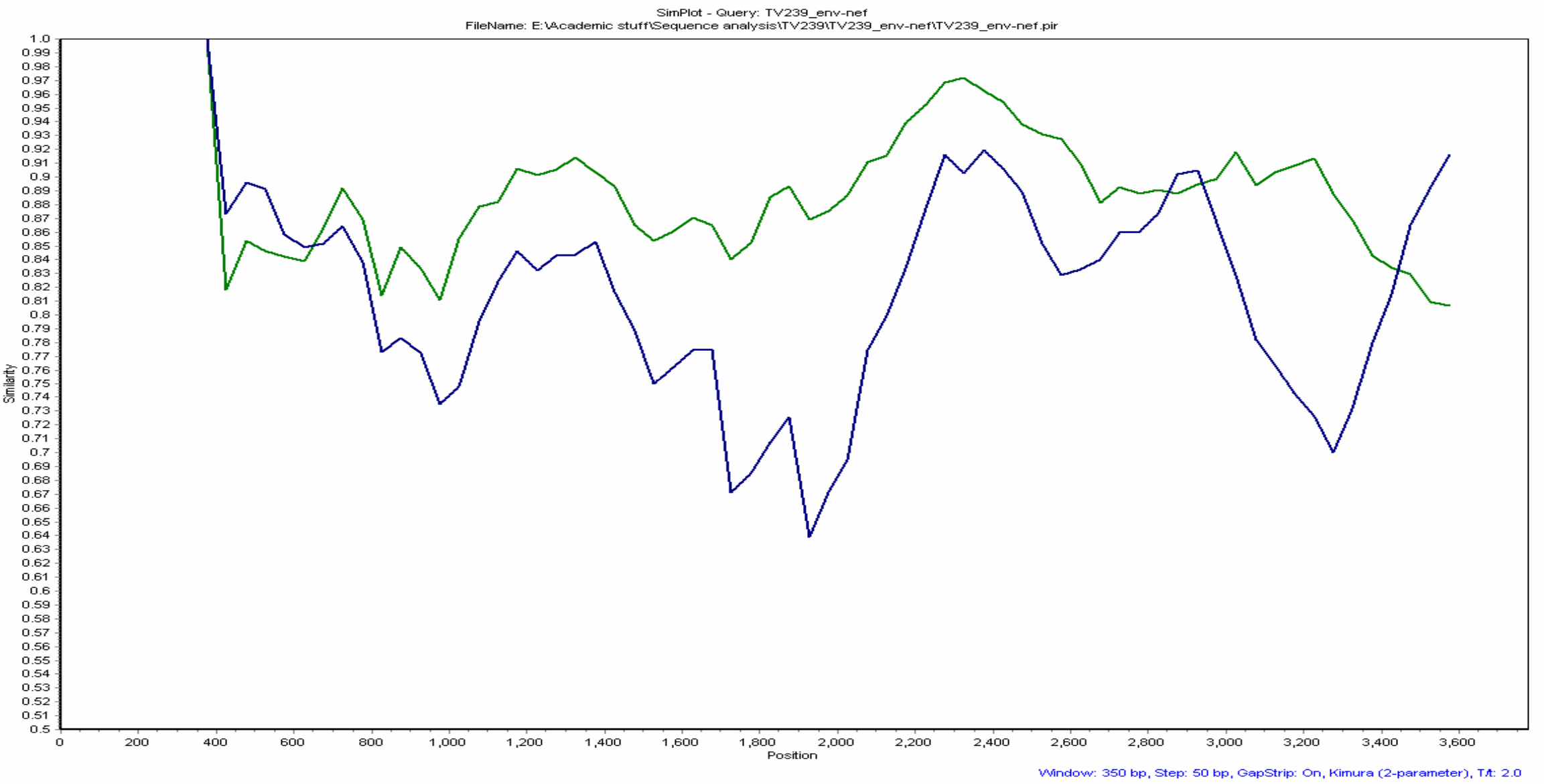

Figure 3.11: Simplot of TV239 env-nef fragment. The horizontal axis represents the position in the fragment and the vertical axis the similarity with viral subtypes against which the sequence of interest (TV239 env-nef) was queried. A window size of 350 bp and a step size of 50 bp were used. The Kimura 2-parameter of nucleotide substitution was used for the analysis. 
SimPlot - Query: T
nalysis:WFLG'S_R84, TV314 \& TV412VAll.aln

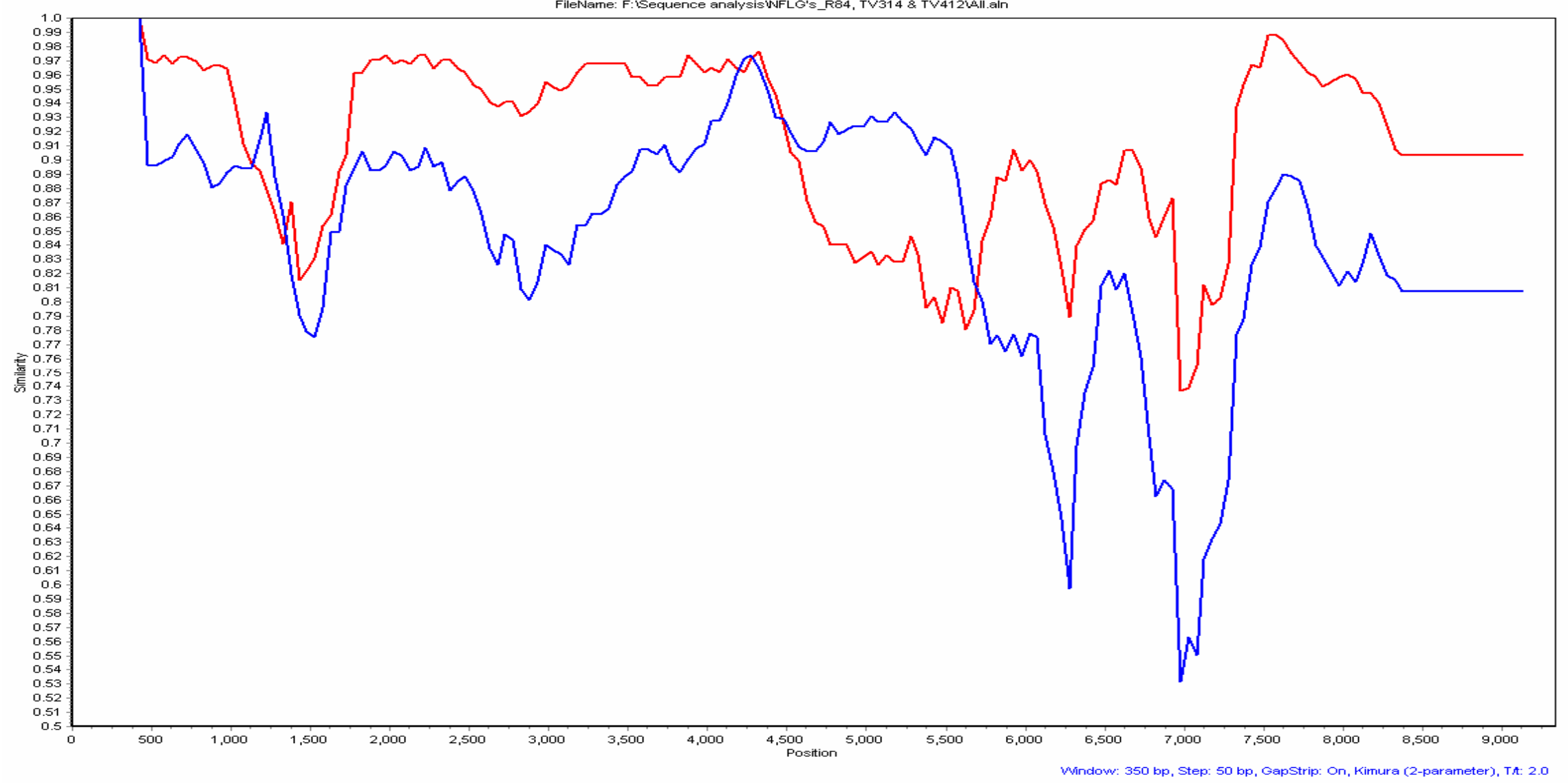

Figure 3.12: Simplot of TV412. The horizontal axis represents the position in the fragment and the vertical axis the similarity with viral subtypes against which the sequence of interest (TV412) was queried. A window size of 350 bp and a step size of 50 bp were used. The Kimura 2-parameter of nucleotide substitution was used for the analysis. 


\subsubsection{Phylogenetic analysis of recombinant breakpoints}

NJ-trees were also drawn of each of the recombinant segments corresponding to the jpHMM breakpoint coordinates (obtained from the subtyping analysis). The breakpoint coordinates of the jpHMM were used, instead of the REGA breakpoints, due to the higher accuracy of the jpHMM analysis ability to calculate breakpoints. The jpHMM uses a jumping approach were the queried sequence can jump between samples in a multiple alignment as a sliding window moves across the fragment, which makes the identification of breakpoints to within a few base pairs much easier. The jpHMM breakpoints of samples TV239 and TV412 are summarized in Table 3.6.

Table 3.6: Breakpoint coordinates of the four samples as was determined by jpHMM analysis.

\begin{tabular}{|c|c|c|c|}
\hline Sample & $\begin{array}{c}\text { Coordinates (Relative to } \\
\text { HXB2) }\end{array}$ & Sample coordinates & Subtype \\
\hline \multirow{4}{*}{ TV239 } & $6195-6335$ & $1-140$ & $\mathrm{C}$ \\
\cline { 2 - 4 } & $6336-8246$ & $141-2050$ & $\mathrm{~A} 1$ \\
\cline { 2 - 4 } & $8247-8532$ & $2051-2337$ & $\mathrm{C}$ \\
\cline { 2 - 4 } & $8533-8846$ & $2338-2651$ & $\mathrm{~A} 1$ \\
\cline { 2 - 4 } & $8847-9146$ & $2652-2951$ & $\mathrm{C}$ \\
\hline \multirow{4}{*}{ TV412 } & $1264-1858$ & $1-594$ & $\mathrm{~A} 1$ \\
\cline { 2 - 4 } & $1859-2095$ & $595-831$ & $\mathrm{D}$ \\
\cline { 2 - 4 } & $2096-5217$ & $832-3953$ & $\mathrm{~A} 1$ \\
\cline { 2 - 4 } & $5218-6130$ & $3954-4866$ & $\mathrm{D}$ \\
\cline { 2 - 4 } & $6131-8254$ & $4867-6990$ & $\mathrm{~A} 1$ \\
\hline
\end{tabular}

A schematic diagram was constructed for each of the recombinant fragments and each recombinant segment in a diagram was assigned a roman numeral (e.g. I, II, and III). See Figure 3.13 A and 3.13 B for the recombinant diagram and trees for the TV239 env-nef fragment. A total of a 100 bootstrap replicates 
was performed on each of the trees. Only bootstrap values greater than 70 percent was included on the trees.

The NJ-tree analysis of the TV239 env-nef fragment revealed that there are five recombinant breakpoints within the sequence/fragment which clusters two different subtypes. The first half of the fragment $(6195-6335)$ clustered with subtype C viruses. The second part (6336-8246) with subtype A1 and the third part with (8247-8532) subtype C again. The fourth (8533-8846) and fifth (8847-9146) segments similarly clustered with subtype A1 and C respectively.

6195

A
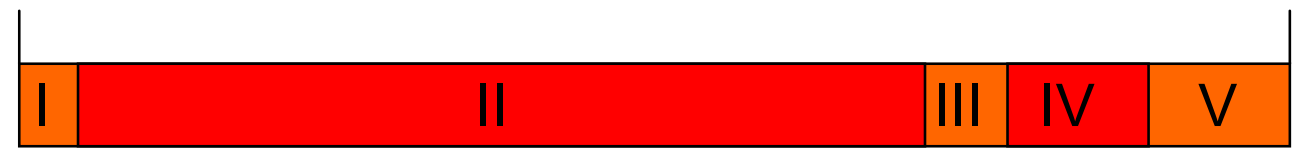

C

A1

C $\quad$ A1

C

Figure 3.13A: A schematic diagram of viral recombination within the TV 239 env-nef fragment. The Roman numerals correspond with the trees in Figure 3.13B. The coordinates on the diagram corresponds with HXB2 coordinates.

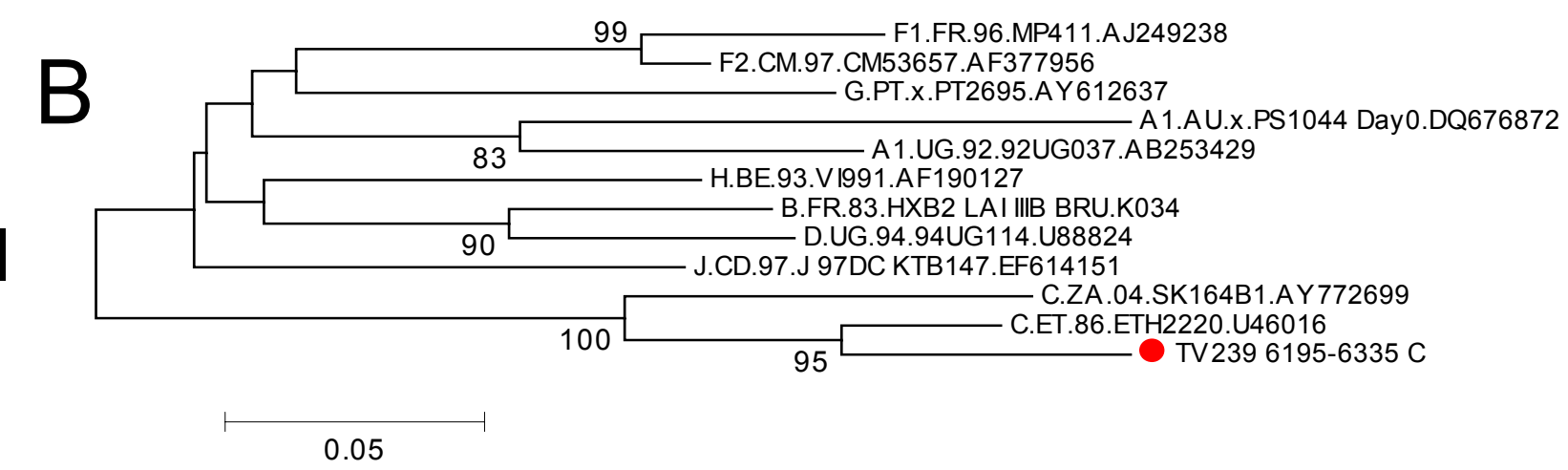

Figure 3.13B: NJ-trees of the different viral recombinant regions of the TV 239 env-nef fragment. The Roman numeral in front of each of the trees corresponds with the numerals in the schematic diagram in Figure 3.13A. The sequence of interest is marked with a red dot. The genetic distance, which corresponds to the length of the branches, is shown in the bottom left hand corner. Only bootstrap values greater than 70 percent are shown on the trees. Figure 3.13B (II - V) continues on page 90. 

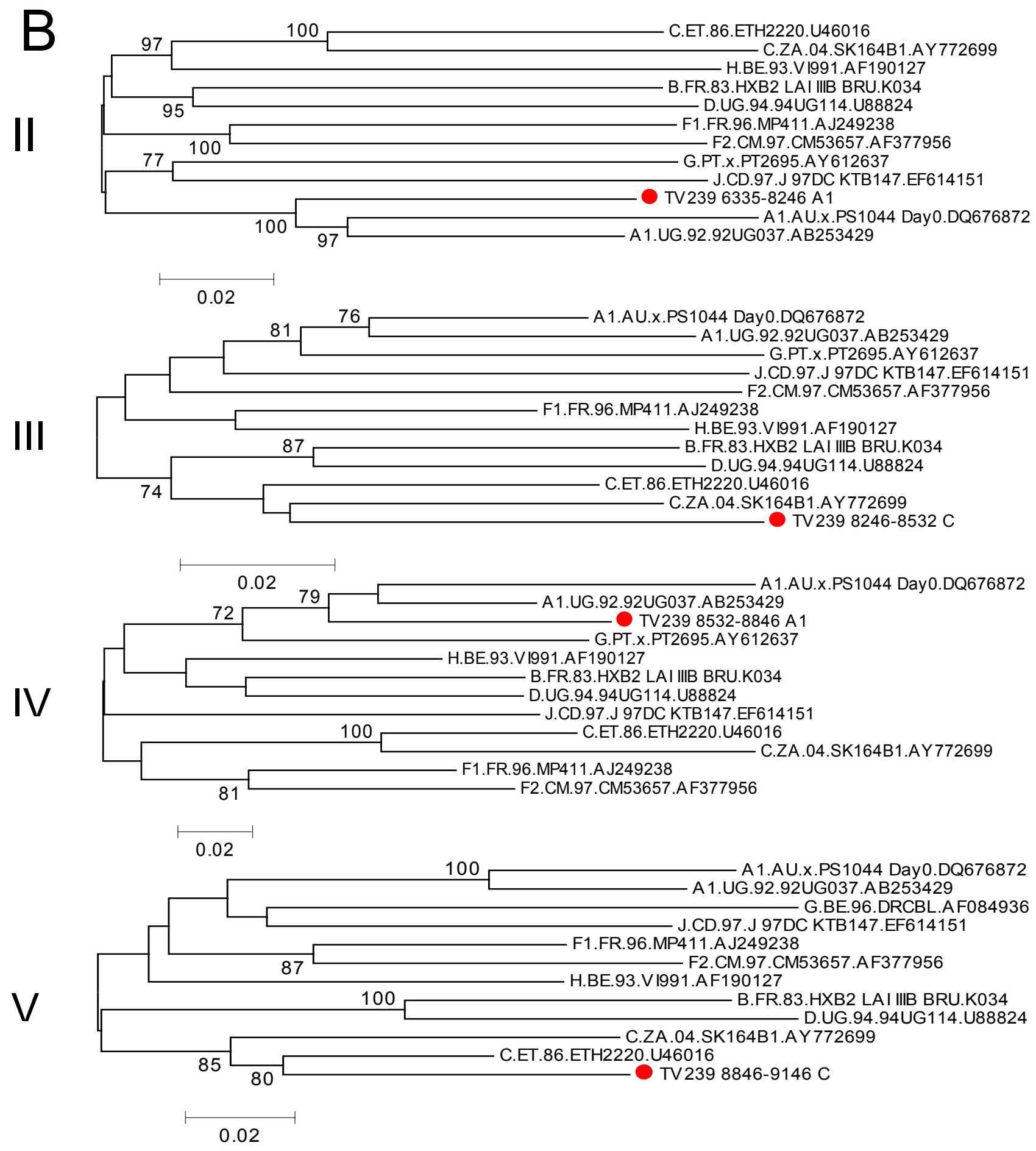

Figure 3.13B (continued): NJ-trees of the different viral recombinant regions of the TV 239 env-nef fragment. The Roman numeral in front of each of the trees corresponds with the numerals in the schematic diagram in Figure 3.13A. The sequences of interest are marked with a red dot. The genetic distance, which corresponds to the length of the branches, is shown in the bottom left hand corner. Only bootstrap values greater than 70 percent are shown on the trees. Figure continued from page 89. 
The results of the viral recombination pattern of TV412 are demonstrated in a similar manner (Figures 3.14A and Figure 3.14B). Similar analysis of the TV412 NJ-trees revealed that this fragment contained five viral segments within the sequenced fragment, which corresponds with two different subtypes. The first (1246 - 1858), third (2096 - 5217) and fifth (6131 - 8254) segments of TV412 clearly clustered with subtype A1 samples in the NJ-trees. The second (1859 - 2095) and fourth (5218 - 6130) segments on the other hand clustered with subtype $D$ viruses in the tree, in particular with D. UG94 . 94UG114 . U88824 from Uganda.

1246

A

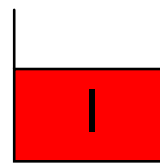
A1

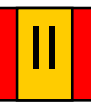

D

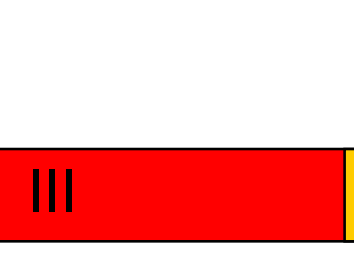

A1 8254

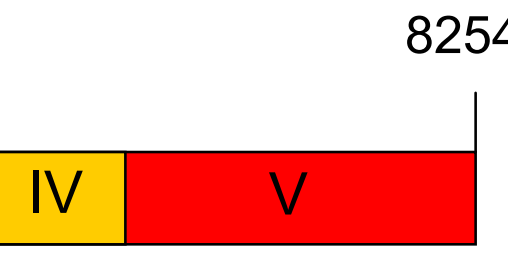

D

A1

Figure 3.14A: A schematic diagram of viral recombination within sample

TV412. The Roman numerals correspond with the trees in Figure 3.15B. The coordinates on the schematic corresponds with that of HXB2.
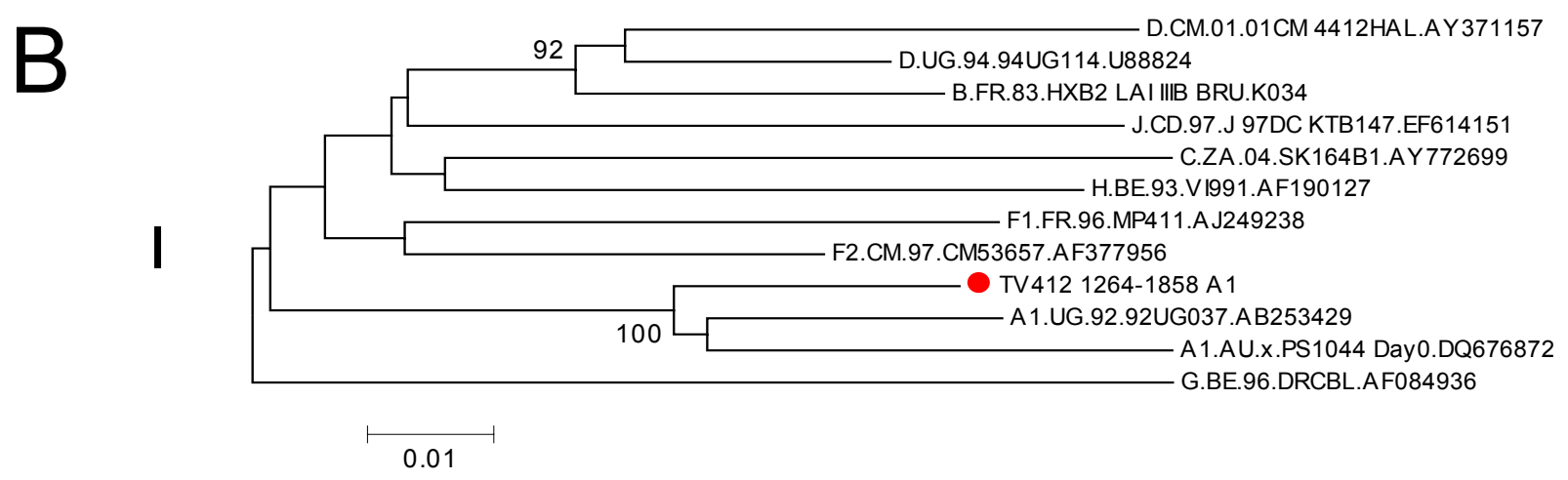

Figure 3.14B: NJ-trees of the different viral recombinant regions of sample TV412. Each Roman numeral corresponds with the numerals in the schematic diagram in Figure 3.14A. Sequences of interest are indicated with a red dot. Only bootstrap values greater than 70 percent are shown on the trees. The genetic distance, corresponding to the branch lengths are shown in the bottom left hand corner. Figure continues on page 92. 
B

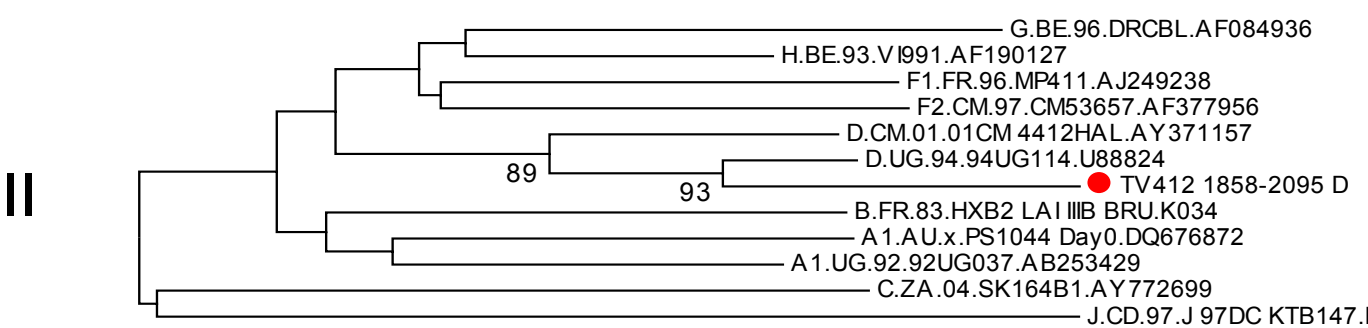
J.CD.97.J 97DC KTB147.EF614151
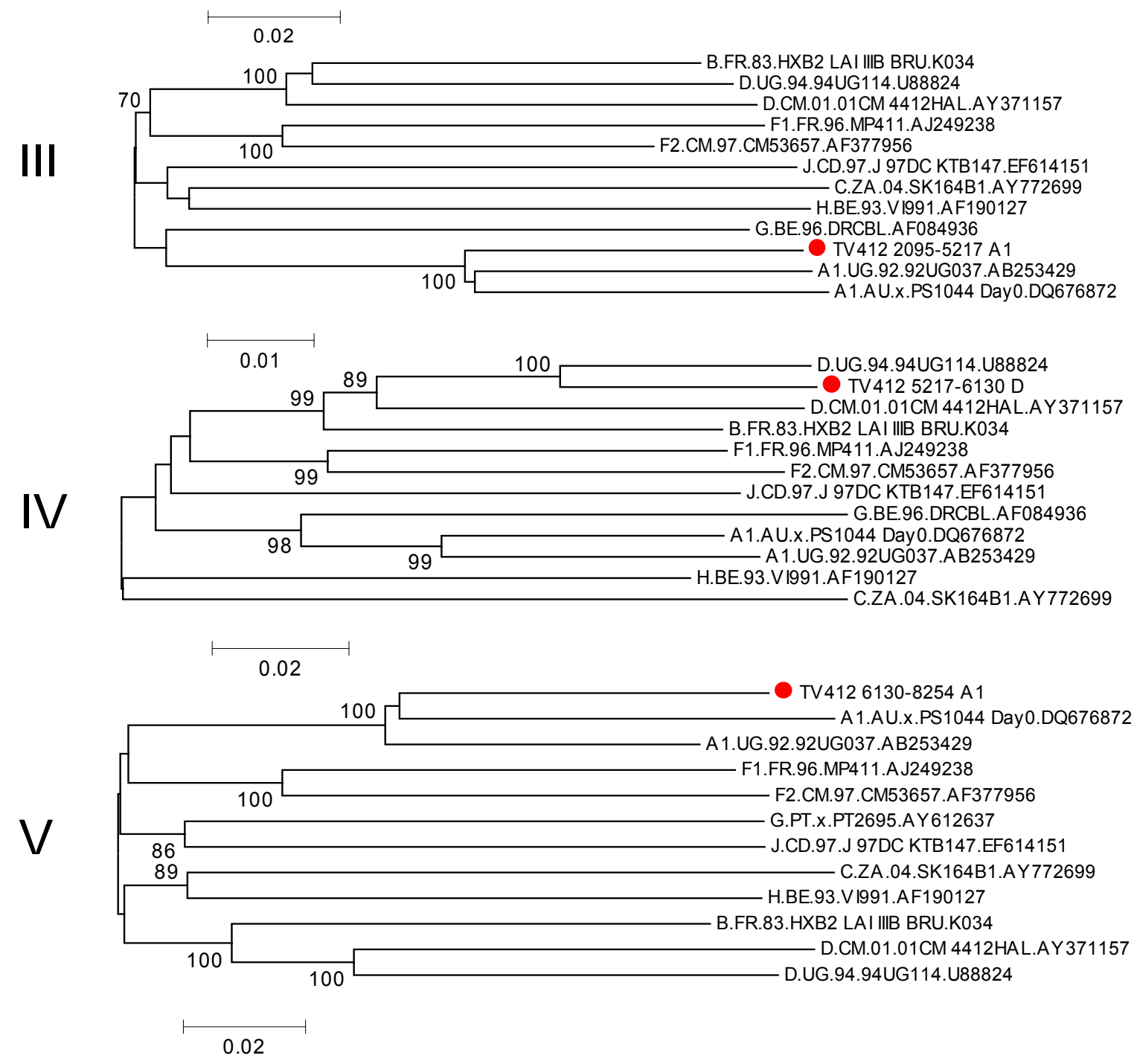

Figure 3.14B continued: NJ-trees of the different viral recombinant regions of sample TV412. Each Roman numeral corresponds with the numerals in the schematic diagram in Figure 3.14A. Sequences of interest are indicated with a red dot. Only bootstrap values greater than 70 percent are shown on the trees. The genetic distance, corresponding to the branch lengths are shown in the bottom left hand corner. Figure continued from page 91. 


\subsection{Analysis of non-recombinant isolates with reference sequences}

The two non-recombinant viral isolates (R84 and TV314) were compared with reference sequences of the same subtype(s). Sample R84 was compared with other subtype B viruses, mostly HIV-1 subtype B isolates from the 1980's and early 1990's. R 84 was aligned with these reference sequences, which was obtained from the LANL database, and a Neighbor-Joining tree (Figure 3.15) was constructed in MEGA v 4.1. TV 314 was compared to other subtype A1 isolates from the LANL database in a similar fashion (Figure 3.16).

Analysis of the phylogenetic analysis of the pure viral fragments revealed that R84 clustered with other subtype B isolates from the United States from the mid 1980's, in particular with sample B.US.83.5082 83, but with a vary low bootstrap support of 50 percent. This sample was isolated in the mid 1980's an thus are suspected to be more closely related to other subtype B viruses from the same period.

The tree with TV314 contains two major clusters, one containing samples of African origin as well as A1 sequences from Sweden and another containing A1 samples that was sampled outside of Africa (mostly in the former Soviet countries or Australia). TV314 clustered with A1 sequences, of African origin, form the mid 1990's till the year 2000. From the analysis it appears that TV314 is more closely related to sample A1.SE .95.UGSE8131. AF107771, with a very high bootstrap support of $87 \%$. This sample was isolated from a patient in Sweden, but clearly clusters amongst other $A 1$ sequences from East African origin, which suggests the introduction and spread of East African A1 isolates into Northern Europe in the distant past. All these assumptions were made basted on the raw phylogenetic data that was obtained from the analysis and more in-depth phylogenetic analysis of TV314 will be needed to verify these claims. 


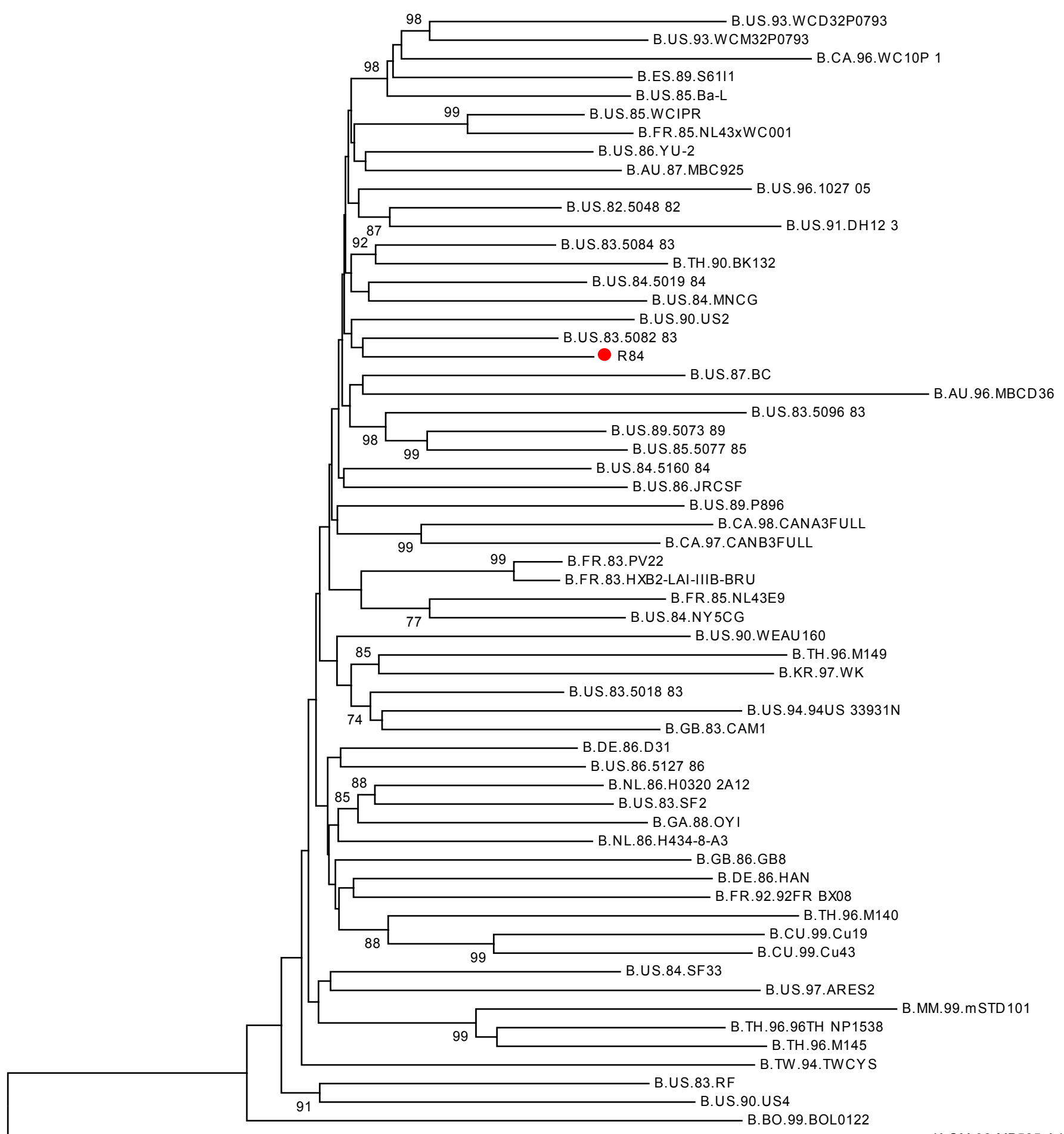

Figure 3.15: A NJ tree exploring the relationship of subtype B HIV-1 isolates with sample R84. The TV sequence is marked with a red dot. The genetic distance, which corresponds to the branch lengths, is shown at the bottom. Bootstrap values greater than 70 percent are shown. 


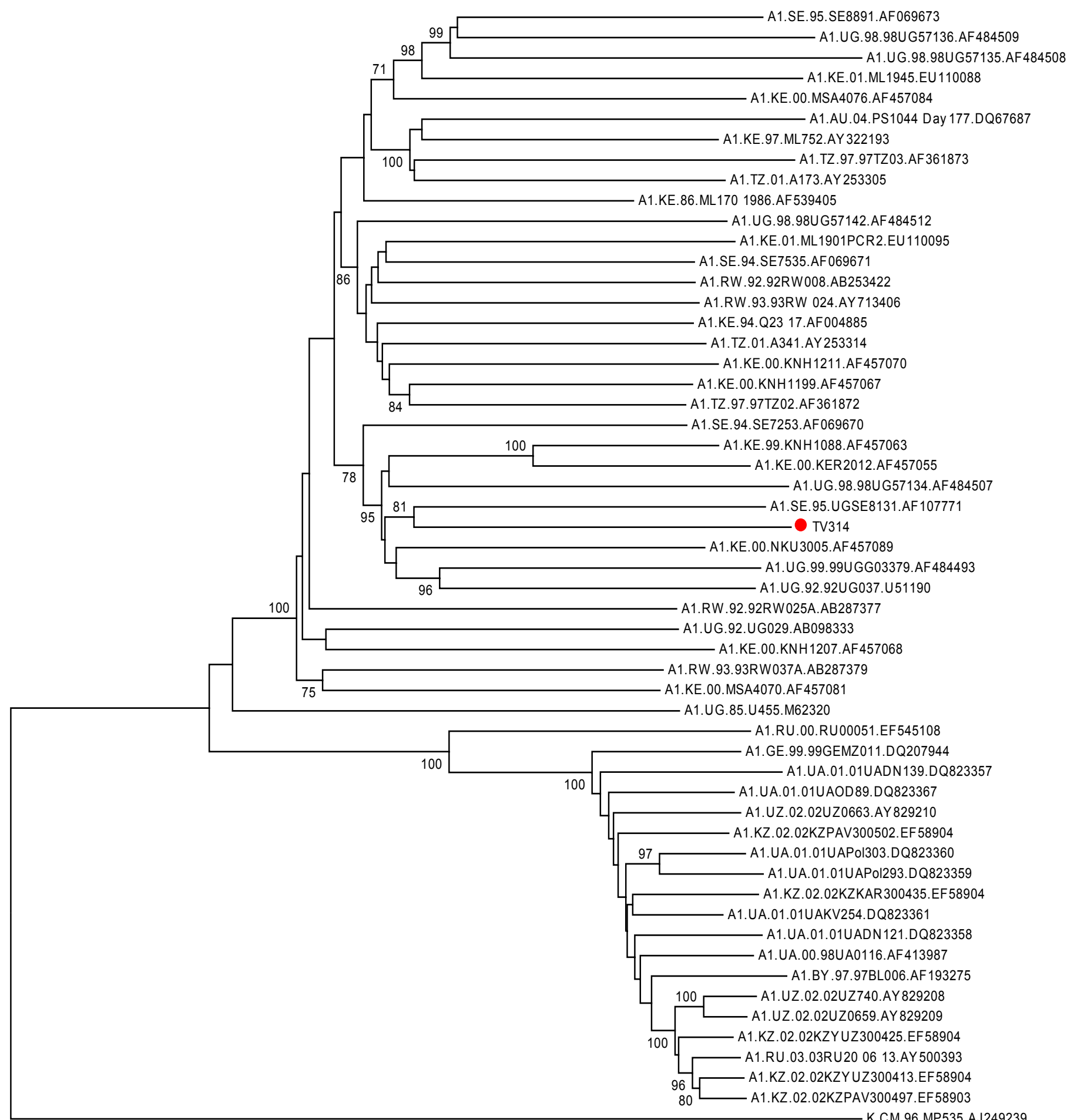

Figure 3.16: A Neighbor-joining tree looking at the relationship between subtypes A1 HIV-1 isolates and sample TV314. The TV sequence is marked with a red dot. The genetic distance, which corresponds to the branch lengths, is shown at the bottom. Bootstrap values greater than 70 percent are shown. 


\section{CHAPTER FOUR - DISCUSSION AND CONCLUSION}

\section{TABLE OF CONTENTS}

Page

DISCUSSION

106

4.1 HIV-1 and HIV characterization in South Africa

4.3 Success of the various amplification assays and sequencing reactions

4.4.2 Discussion of near full-length sequence results

4.4.2.1 R84 and other subtype $B$ viruses in South Africa

4.4.2.2 TV239 and other HIV recombinants from

South Africa

4.4.2.3 Subtype A's in South Africa and TV314 


\section{CHAPTER FOUR - DISCUSSION AND CONCLUSION}

\section{DISCUSSION}

The objective of this study was to characterize non-subtype $C$ isolates, from the greater Cape Town Metropolitan area. This study indicated that nonsubtype C HIV-1 isolates, including subtypes $B, A 1$ and $A 1$ recombinant viruses, are circulating within the Cape Town area.

\subsection{HIV-1 and HIV characterization in South Africa}

The first documented cases of HIV-1 infection in South Africa occurred in 1982 [Ras et al, 1983]. Unlike the rest of sub-Sahara Africa, the HIV-1 epidemic in South Africa was mostly associated with the homosexual population [Sher, 1989]. The isolates were later characterized as subtype $B$ and D viruses [Becker et al, 1995; Engelbrecht et al, 1995]. Subtype B and D viruses were probably introduced into the country by homosexual men through international travel. By the start of the 1990's a new epidemic of HIV started to occur within the country amongst heterosexual individuals, which was largely associated with members of the indigenous black population [Williamson et al, 1995]. Viruses that were isolated from heterosexual individuals of the second epidemic mostly belonged to HIV-1 subtype C [van Harmelen et al, 1997]. The second epidemic quickly overtook the first initial epidemic in the early 1990's and today the heterosexually transmitted dominates the HIV epidemic within the country [McCutchan et al, 1996], with as many as 6.2 million people infected [UNAIDS, 2008].

To date several papers on a wide range of HIV-1 subtypes have been published within South Africa on subgenomic fragments or near full-length sequences (Tables 1.2 and 1.3). These viruses were isolated from several geographical locations within the country. The following section will take a look at the publications. 
Subtype C HIV-1 accounts for nearly $95 \%$ of all infections within the country and subtype $C$ viruses have been characterized on several occasions. Of the 3884 South African full or partial sequences in the LANL database, 3706 or 95.4\% are subtype C (Figure 1.8). One should note that these figures may be misleading as several sequences may be from the same patient sample. The rest of the sequences represents subtype $B$ and $D$ isolates, which has been described in the past in association with homosexual mode of transmission [Engelbrecht et al, 1994; Becker et al, 1995], and other subtypes and recombinant forms.

Two hundred and sixty five full-length HIV-1 sequences from 8 independent studies have been described within the country. The majority of these sequences represent subtype C isolates [van Harmelen et al, 2001; Papathanasopoulos et al, 2002; zur Megede et al, 2002; Papathanasopoulos et al, 2003; Hunt et al, 2003; Rousseau et al, 2006]. Only 11 full-length sequences of non-subtype $C$ isolates have been described to date within South Africa. These include 5 subtype D isolates [Loxton et al, 2005; Jacobs et al, 2007], one subtype B [Rousseau et al, 2006], one subtype A1 [Rousseau et al, 2006], three AC recombinants [Papathanasopoulos et al, 2002; Rousseau et al, 2006] and one complex viral form containing subtypes A, C, D, G and K segments [Papathanasopoulos et al, 2002].

As with the characterization of full-length genomes from South Africa, most partial sequence fragments also represents subtype $C$ isolates [Bredell et al, 1998; Engelbrecht et al, 2001; Scriba et al, 2002; Gordon et al, 2003; Bessong et al, 2005; Bell et al, 2007]. Several non subtype C viruses, apart from the subtype $B$ and $D$ viruses that was mentioned earlier [Engelbrecht et al, 1994; Becker et al, 1995], have been identified. These include, an CRF01_AE isolate [van Harmelen et al, 1997], subtype A isolates [Jacobs et al, submitted; Bredell et al, 2002], a $G$ and one CRF02_AG isolate [Bredell et al, 2002; Jacobs et al, 2008], CD recombinants [Bredell et al, 2002; Gordon et al, 2003], an $\mathrm{F} 1$ isolate [Jacobs et al, submitted], CA recombinants [Bredell et al, 2002] and a $\mathrm{CH}$ recombinant [Jacobs et al, submitted]. Some of these isolates are extremely rare, such as $\mathrm{F} 1, \mathrm{CH}$ recombinants and subtype $\mathrm{G}$, and 
are mostly confined to areas of Central Africa e.g. the DRC (Figure 1.8) [Geretti, 2006].

\subsection{Full genome characterization of HIV}

HIV sequences can be grouped into one of the 9 subtype by either characterization of small gene fragments throughout the genome or by characterizing the full HIV genome. The use of the first method has been well established over the years [Swanson et al, 2003]. This method does however have its own drawbacks as some recombination events are so small and can be missed in such an approach. With these limitations of partial genome sequencing the focus was shifted to the characterization of full-length HIV-1 genomes. Improvements in nucleic acid amplification technology, which permits the amplification of large gene fragments of $(9-36 \mathrm{kbp})$ now enables researchers to amplify and characterize full-genomes of HIV much easier. This procedure was first described in HIV-1 research by Mika Salminen and co-workers in 1995 [Salminen et al, 1995a]. Since then the value of fullgenome sequencing of HIV has been repeatedly demonstrated. The subtype E viruses, which were isolated and sequenced in the early 1990 in Southeast Asia, were initially termed as subtype $E$ based on sequences from the env gene [Carr et al, 1996; Gao et al, 1996]. Full genome characterization of these isolates reveled that the isolates were recombinant in nature with subtype $A$ in the gag and subtype $\mathrm{E}$ in the env regions. The Z321 strain from Zaire, which was one of the earliest identified strains HIV-1, was initially thought to have been subtype $A$ based on env sequences, but with full genome amplification it was found to be an AG recombinant [Choi et al, 1997]. This emphasizes the importance of full genome amplification and sequencing of HIV isolates, especially when viral recombination is suspected.

Full genome characterization of HIV-1 can either be performed on, proviral DNA or RNA (isolated from PBMC) or viral RNA from blood plasma. In the HIV database of LANL there are only 1404 HIV-1 sequences which are greater than $8 \mathrm{kbp}$ in length (any continues HIV fragment larger than $8000 \mathrm{bp}$ is considered to be a near full-length genome) [http://www.hiv.lanl.gov/]. The 
majority of these near full-length sequences were generated by using proviral DNA from either peripheral blood mononuclear cells (PBMC) or either cultured cells. A standard protocol for the characterization of near full-length sequences from viral RNA was recently developed [Nadai et al, 2008]. Viral RNA represents the current replicating viral population within a patient and thus may offer more pathogenic and phylogenetic significance than with DNA isolates from PBMC's [Wei et al, 1995].

\subsection{Success of the various amplification assays and sequencing reactions}

The amplification of the near-full length genomes were all unsuccessful and non specific products were obtained. This might be due to un-optimized PCR assays, old DNA or the use of unspecific primer pairs.

All of the other PCR's that were performed in this study were successful, except for the three subgenomic fragments of TV546, the env fragments of TV480 and the LTR-gag amplification assays of TV239, TV314 and TV412. PCR failures might be due to the high degree of sequence variation in HIV-1. Subtype specific primers could have been design but due to sample limitations was abandoned. TV480 and TV546 have been identified as possible $\mathrm{CJ}$ and $\mathrm{CG}$ recombinant viruses in a previous study [Jacobs et al, submitted]. The primer sets that were used by Swanson and co-workers [Swanson et al, 2003] might not have been specific enough for these two samples. When doing PCR's it is also important to optimize each assay for greater yield in product.

The sequencing of the positive PCR products was all successful with the exception of TV340 gag p24 and TV239. Multiple peaks in the electrophenogram made it impossible to obtain a reliable sequence for TV340 gag p24. The failure of the sequencing of TV239 which produced a $600 \mathrm{bp}$ gap in the pol-vif region of TV239 might be due to frequent recombination events within this region of the genome. 


\subsection{HIV subtype results of the present study}

This section will discuss the HIV subtype results that were generated in this study. In the first section the results of the characterization of the various subgenomic regions will be discussed. The second section will discuss the results that were obtained in the near full-length characterization of four samples.

\subsubsection{Discussion of the various subgenomic fragments}

The characterization of small subgenomic regions throughout the HIV genome to subtype an isolate or identify possible recombinant has been widely used in the past [Swanson et al, 2003]. The method that was developed by Swanson and co-workers targeted specific regions that is important in HIV antibody/antigen diagnostic assays and commercially available viral load assays. Theses regions are generally highly conserved and the PCR's that was developed for this study was able to detect most HIV-1 groups and subtypes.

In the present study subtype $\mathrm{C}, \mathrm{F} 1, \mathrm{~A} 1$, and $\mathrm{B}$ isolates were identified with this method of genome characterization. Several recombinant HIV viruses were also identified, including $A D, C A, A G$ and other possible $A 1$ recombinant forms. Sample TV546 was not amplifiable with the primer sets that were used. This sample was identified as a subtype $G$ or subtype $C$ isolate on previous occasion [Jacobs et al, submitted]. This illustrates the need for subtype specific primers when working with rare viral subtypes or recombinants forms.

The V3 region of TV515 was characterized on a previous occasion [Jacobs et al, submitted]. These gag p24, pol-integrase and env gp41 sequences of TV515 will represent the second sequenced set of F1 sequences from South Africa, though from the same patient. Sample TV340 were previously classified [Jacobs et al, submitted] as a subtype A isolate. This sample was isolated from a patient in the Cape Town, which became infected in the DRC (Table 2.4). Through analysis of the pol and env subgenomic regions this 
isolate was classified as an AG recombinant, with the pol fragment belonging to subtype $G$ and the env fragment to subtype $A 1$.

Similarly analysis of TV441 was identified as a subtype A1 isolate in the same study. The analysis of the present study indicated that this sample is a possible AC recombinant with the gag p24 and pol-integrase regions clustering with subtype $C$ viruses and the env gp41 region with subtype $A$ viruses. It might be concluded that subgenomic characterization of isolates may yield more accurate results when several regions throughout the genome of HIV-1 is targeted.

Though these methods of HIV genome characterization may give an indication of the viral subtype of a particular isolate or identify viral recombination, only full genome analysis of samples will allow one to make a safe and accurate assumption on the subtype and recombination of particular isolates, as recombination can occur in the regions between the three subgenomic fragments characterized.

\subsubsection{Discussion of near full-length sequence results}

\subsubsection{R84 and other subtype B viruses in South Africa}

Subtype B HIV are typically found amongst homosexual men in North America and Europe. Since the outbreak of the epidemic, subtype $B$ has also established itself in South America, parts of Asia, South Africa, and in Australia [Leitner et al, 1996]. In South Africa subtype B, along with subtype $D$, viruses were isolated and later sequenced from homosexual men in the mid 1980's and early 1990's [Becker et al, 1995; Engelbrecht et al, 1995]. To date only a few cases have been reported where subtype $B$ viruses were isolated from heterosexual individuals [van Harmelen et al, 1997; van Harmelen et al, 1999]. Heterosexual transmittion of subtype $B$ has also been reported in other areas of the world [Lara et al, 1997; Hayman et al, 2001; Cleghorn et al, 2000]. Subtype B HIV has also been reported in MTCT cohorts from Cape Town [Jacobs et al, submitted]. Only one full-length sequence of a 
South African subtype B virus, which was isolated from a heterosexual female, have been characterized to date [Rousseau et al, 2006].

A full-length subtype B sequence, which was isolated from a homosexual caucasian male in the mid 1980's, were characterized in this study. Amplification and sequencing of the isolate produced an $8913 \mathrm{bp}$ fragment, which represents the second near full-length sequence of a subtype $B$ isolate from South Africa. This subtype B isolate along with the subtype D viruses, which has been characterized in the past [Loxton et al, 2005; Jacobs et al, 2007], represent the only near full-length sequences of the first initial homosexual HIV epidemic within the country. This near full-length sequence will greatly improve our knowledge and understanding of the initial HIV epidemic within the country.

As was mentioned previously, heterosexual transmittion of subtype $B$ have been reported in the past [van Harmelen et al, 1997; van Harmelen et al, 1999; Jacobs et al, submitted]. It would be reasonable to assume that if the circulation of subtype $B$ viruses continues to increase and co-circulate with subtype $\mathrm{C}$ amongst the heterosexual population of South Africa that this could create a good opportunity for viral recombination to occur and the possible rise of $\mathrm{CB}$ recombinants within the region.

\subsubsection{TV239 and other HIV recombinants from South Africa}

Sample TV239 were isolated from a South African male, which presented with tuberculosis and a low CD4 cell count of 64. TV239 was characterized previously, through amplification and sequencing of a small (300 bp) fragment of the env gene, as a subtype A HIV-1 isolate. With the full genome analysis of this isolate it became apparent that the TV239 isolate was an AC recombinant. This only stresses the importance of full-genome characterization of HIV-1 isolates, as such recombination events can be missed with the amplification of smaller fragments.

Only three full-length sequences of $A C$ recombinant viruses have been described in South Africa to date [Papathanasopoulos et al, 2002; Rousseau 
et al, 2006]. Through phylogenetic analysis of the two AC recombinants that was described by Rousseau and colleagues revealed that the $A$ fragments of the two recombinants were more closely related to sub-subtype $A 1$. The one $A C$ recombinant of Papathanasopoulos and co-workers was classified with the $A$ recombinant fragments clustering with subtype $A 2$ isolates as with $A 1$ sub-subtypes of subtype A HIV-1 isolates. All the subtype A recombinant fragments as well as those of TV239 was more closely related to other subtype A sequences from East African. TV239 thus represents the fourth near full-length AC recombinant that has been described in South Africa to date, and only the third $\mathrm{A} 1 \mathrm{C}$ recombinant.

As subtype A continue to spread within the region of Southern Africa and cocirculate with subtype $\mathrm{C}$ and other viral subtype one can expect and increase in the prevalence of these recombinant forms in the future.

\subsubsection{Subtype A's in South Africa and TV314}

TV314 was obtained from an asymptomatic South African male. Near fulllength genome characterization revealed that the isolate was subtype $A 1$ with no trace of recombination within the sequenced fragment. A total of 37 sequences of subtype A viruses from South Africa can be found in the LANL database, but only one full-length subtype A1 sample have been described to date [Rousseau et al, 2006]. The near full-length subtype A1 sequence that was described previously within the country was isolated form a heterosexually-infected female of Zulu or Xhosa ethnicity. The gag portion of this isolate was more closely related to the subtype A gag part that is characteristic of CRF01_AE isolates.

TV314 was more closely related to an A1 sequence that was characterized from Sweden, A1.SE.95.UGSE8131.AF107771, but these two sequences were grouped together with other subtype A1 sequences from East African origin. The dataset that was used for the analysis contained four sequences from Sweden, all of whom clustered amongst the other East African sequences. It may be concluded that this strain was introduced into Northern Europe via international travel or emigration. 


\subsubsection{TV412 and other AD recombinants from Kenya}

Sample TV412 were isolated from an African male, which presented with chronic staphylococcal skin sepses. The patient's immune system was severely depleted with a CD4 count of 71 . According to Hospital records the patient became infected with HIV in Kenya. Full-genome amplification was performed, but was insufficient for sequencing. Amplification was performed in four overlapping fragments, three of which were successful. This resulted in a 7008 bp sequenced fragment, stretching from 1246 - 8254 (relative to HXB2 coordinates). TV412 was classified, with the use of REGA and jpHMM subtyping, as a subtype $A 1$ isolate based on the $300 \mathrm{bp} \mathrm{V} 3$ sequence that was characterized within the department [Jacobs et al, submitted]. In this study, through the characterization of a larger fragment (7 kbp) the sample classified as an $A D$ recombinant. This stresses the importance of full-genome characterization of samples in order to make an accurate and informed conclusion concerning an isolates subtype status.

Subtype A and D HIV co-circulate in large numbers within the East African country of Kenya. Subtype A and D respectively represents 74.4 and 10.6 percent of the subtypes circulating within the country (Figure 1.8). This cocirculation of these two subtype, which are also found in large numbers in other East African countries (e.g. Uganda and Tanzania), presents a good opportunity for viral recombination. Today, $A D$ recombinant viruses comprise nearly $1.9 \%$ of the viruses that are sampled. Similarly, Tanzania and Uganda have reported $A D$ recombinant levels of 1.1 and 2.2 percent respectively (Figure 1.8). Recent data on co-infection of individuals with subtypes $A$ and $D$ showed that there are an ongoing generation and selection for $A / D$ recombinant forms [Songok et al, 2004]. 


\section{CONCLUSION}

This work represents partial gag, pol and env and near-full length sequences of non-subtype C HIV-1 sequences from the Tygerberg Hospital, Cape Town, South Africa. Phylogenetic analysis of the sequenced data revealed the presence of subtype $A, B, F 1$ as well as $A C$ and $A D$ recombinant viruses within the region. The two near full-length sequences of the recombinant viruses constitute two new unique recombinant HIV-1 forms. The data that was gathered in this study will greatly improve our knowledge of subtype distributions within the country. Due to the impact that HIV genetic diversity might have on vaccine design and development, as well as HIV diagnosis and the treatment of patients with antiretroviral therapeutic drug, ongoing research into the epidemiology and spread of HIV subtypes and recombinants within South Africa are needed. 


\section{CHAPTER FIVE - REFERENCE LIST}

Abecasis A, Vandamme A-M, Lemey P. Sequence Alignment in HIV Computational Analysis pp. 2-16 in HIV Sequence Compendium 2006/2007. Edited by: Thomas Leitner T, Foley B, Hahn B, Marx P, McCutchan F, Mellors J, Wolinsky S, Korber B. Published by: Theoretical Biology and Biophysics Group, Los Alamos National Laboratory, Los Alamos, NM. LA-UR 07-4826. 2007

Achong G, Mansell PWA, Epstein MA, and Clifford P. An unusual virus in cultures from a human nasopharyngeal carcinoma. NJCI. 1971. 42: 299-307

Altschul SF, Gish W, Miller W, Myers EW, and Lipman DJ. Basic Local Alignment Search Tool. Journal of Molecular Biology. 1990. 215: 403-410

Ammann AJ, Abrams D, Conant M, Chadwin D, Cowan M, Volberding P, Lewis $B$, and Casavant $C$. Acquired immune dysfunction in homosexual men: immunologic profiles. Clinical Immunology and Immunopathology. 1983. 27: 315-325

Andersson S, Norrgren H, Dias F, Biberfeld G, and Albert J. Molecular characterization of human immunodeficiency virus (HIV)-1 and -2 in individuals from Guinea-Bissau with single or dual infections: predominance of a distinct HIV-1 subtype A/G recombinant in West Africa. Virology. 1999. 262: 312-320

Anderson JP, Rodrigo AG, Learn GH, Madan A, Delahuntry C, Coon M, Girard M, Osmanov S, Hood L, and Mullins Jl. Testing the Hypothesis of a Recombinant Origin of Human Immunodeficiency Virus Type 1 Subtype E. Journal of Virology. 2000. 74: 10752-10765

Ariën KK, Vanham G, and Arts EJ. Is HIV-1 evolving to a less virulent form in Humans? Nature Reviews Microbiology. 2007. 5: 141-151 
Bachmann MH, Delwart EL, Shpaer EG, Ligenfelter P, Singal R, and Mullins JI. Rapid genetic characterization of HIV type 1 strains from four World Health Organization-sponsored vaccine evaluation sites using a heteroduplex mobility assay. WHO Network for HIV Isolation and Characterization. AIDS Research and Human Retroviruses. 1994. 10: 1345-1353

Baldauf SL. Phylogeny for the faint of heart: a tutorial. TRENDS in Genetics. 2003. 19: $345-351$

Barre-Sinoussi F, Chermann JC, Rey F, Nugeyre MT, Chamaret S, Gruest J, Dauguet C, Axler-Blin C, Brun-Vezinet F, Rouzioux C, Rozenbaum W, and Montagnier L. Isolation of a T-Lymphotropic retrovirus from a patient at risk for Acquired Immune Deficiency Syndrome (AIDS). Science. 1983. 220: 868-871

Bebenek K, Abbotts J, Roberts JD, Wilson SH, and Kunkel TA. Specificity and mechanism of error-prone replication by human immunodeficiency virus-1 reverse transcriptase. Journal of Biological Chemistry. 1989. 264: 1694816956

Becker ML, de Jager G, and Becker WB. Analysis of partial gag and env gene sequences of HIV type 1 strains from Southern Africa. AIDS Research and Human Retroviruses. 1995. 11 (10): 1265-1267

Becker MLB, Spracklen FHN, and Becker WB. Isolation of a Lymphadenopathy associated virus from a patient with acquired immune deficiency syndrome. South African Medical Journal. 1985. 68: 144-147

Bell CM, Connell BJ, Capovilla A, Venter WDF, Stenves WS, and Papathanasopoulos MA. Molecular Characterization of the HIV Type 1 Subtype C Accessory Genes vif, vpr, and vpu. AIDS Research and Human Retroviruses. 2007. 23: 322-330

Bessong PO, Obi CL, Cilliers T, Choge I, Phoswa M, Pillay C, Papathanasopoulos $\mathrm{M}$, and Morris L. Characterization of Human Immunodeficiency Virus Type 1 from a Previously Unexplored Region of 
South Africa with a High HIV Prevalence. AIDS Research and Human Retroviruses. 2005. 21: 103-109

Boyer JC, Bebenek K, Kunkel TA. Unequal human immunodeficiency virus type 1 reverse transcriptase error rates with RNA and DNA templates. Proceedings of the National Academy of Sciences. 1992. 89: 6919-6923

Bredell H, Hunt G, Casterling A, Cilliers T, Rademeyer C, Coetzer M, Miller S, Johnson D, Williamson C, and Morris L. HIV-1 Subtype A, D, G, AG and Unclassified Sequences identified in South Africa. Aids Research and Human Retroviruses. 2002. 18; 9: 681-683

Bredell H, Williamson C, Sonnenberg P, Martin DJ, and Morris L. Genetic characterization of HIV type 1 from migrant workers in three South African gold miners. AIDS Research and Human Retroviruses. 1998. 14: 677-684

Buonaguro L, Tornesello ML, and Buonaguro FM. Human Immunodeficiency Virus Type 1 Subtype Distribution in the Worldwide Epidemic: Pathogenetic and Therapeutic Implications. Journal of Virology. 2007. 81: 10209-10219

Carr JK, Salminen MO, Albert J, Sanders - Buell E, Gotte D, Birx DL, and McCutchan FE. Full genome sequences of human immunodeficiency virus type 1 subtype $G$ and A/G intersubtype recombinants. Virology. 1998. 247: 22-31

Carr JK, Salminen MO, Koch C, et al. Full-length sequence and mosaic structure of a human immunodeficiency virus type 1 isolate from Thailand. Journal of Virology. 1996. 70: 5935-5943

CDC. 'Kaposi's Sarcoma (KS), Pneumocystis Carinii Pneumonia, and Other Opportunistic Infections (01): Cases Reported to CDC as of July 8'. 1982 www.cdc.gov/hiv/topics/surveillance/resources/reports/surveillance82.pdf

CDC. Kaposi's sarcoma and Pnerumocycsits pneumonia among homosexual men - New York City and California. Morbitity and Mortality Weekly Report. 1981. 30: 305-308 
Chan D and Kim P. HIV entry and its inhibition. Cell. 1998. 93: 681-684

Chan DC, Fass D, Berger JM, and Kim PC. Core Structure of gp41 from the HIV Envelope Glycoprotein. Cell. 1997. 89: 263-273

Choi DJ, Dube S, Spicer TP, Slade HB, Jensen FC, and Poiesz BJ. HIV type 1 isolate Z321, the strain used to make a therapeautic HIV type 1 immunogen, is intersubtype recombinant. AIDS Research and Human Retrovirus. 1997. 13: 357-361

Cichutek K and Norley S. Lack of immune suppression in SIV infected natural hosts. AIDS. 1993. 7(suppl1): S25-S35

Cleghorn FR, Jack N, Carr JK, Edwards J, Mahabir B, Sill A, McDanal CB, Connolly SM, Goodman D, Bennetts RQ, O'Brien TR, Weinhold KJ, Bartholomew C, Blattner WA and Greenberg ML. 2000. A distinctive clade B HIV type 1 is heterosexually transmitted in Trinidad and Tobago. Proceedings of the National Academy of Science. 97: 10532-10537.

Clumeck N, Mascart-Lemone F, de Maubeuge J, Brenez D, and Marcelis L. Acquired immune deficiency syndrome in Black Africans. Lancet. 1983.1: 642

Curran JW, Lawrence DN, Jaffe H, Kaplan JE, Zyla LD, Chamberland M, Weinstein R, Lui KJ, Schonberger LB, and Spira TJ. Acquired immunodeficiency syndrome (AIDS) associated with transfusions. New England Journal of Medicine. 1984. 310; 69-74

De Cock KM, Adjorlolo KMG, Ekpini E, Sibailly T, Kouadio J, Maran M, Brattegaard K, Vetter KM, Doorly R, and Gayle HD. Epidemiology and transmission of HIV-2: why there is no HIV-2 pandemic. Journal of American Medical Association. 1993. 270: 2083-2086

De Leys R, Vanderborght B, Vanden Haesevelde M, Heyndrickx L, van Geel A, Wauters C, Bernaerts R, Saman E, Nijs P, Willems B, Taelman H, Van der Groen G, Piot P, Tersmette T, Huisman JG, and Van Heuverswyn H. Isolation and partial characterization of an unusual human immunodeficiency retrovirus 
from two persons of west-central African origin. Journal of Virology. 1990. 64: 1207-1216

Delwart EL, Shpaer EG, McCutchan FE, Louwagie J, Grez M, RübsamenWaigmann H, Mullins Jl.. Genetic relationships determined by a DNA heteroduplex mobility assay: Analysis of HIV-1 env genes. Science. 1993. 262:1257-1261

de Oliveira T, Engelbrecht S, Janse van Rensburg E, Gordon M, Bishop K, zur Magede J, Barnett SW, and Cassol S. Variability at Human Immunodeficiency Virus Type 1 Subtype $C$ Protease Cleavage Sites: an Indication of Viral Fitness? Journal of Virology. 2003. 77: 9422-9430

de Oliveira T, Deforche K, Cassol S, Salminen M, Paraskevis D, Seebregts C, Snoeck J, Janse van Rensburg E, Wensing AMJ, van de Vijver DA, Boucher $\mathrm{CA}$, Camacho R, and Vandamme A-M. An automated genotyping system for analysis of HIV-1 and other microbial sequences. Bioinformatics Applications Note. 2005. 21: 3797-3800

Derdeyn CA, Becker JM, Bibollet-Ruche F, Mokili JL, Muldoon M, Denham SA, Heil ML, Kasolo F, Musonda R, Hahn BH, Shaw GM, Korber BT, Allen S, and Hunter E. Envelope-constrained nuetralizationsensitive HIV-1 after heterosexual transmission. Science. 2004. 303: 2019-2022

Department of Health, Republic of South Africa. National HIV and Syphilus Antenatal sero-prevalence survey in South Africa. 2004. Pretoria, South Africa: Directorate Health systems Research, Department of Health; 2005.

Dicker IB, Samanta HK, Li Z, Hong Y, Tian Y, Banville J, Remillard RR, Walker MA, Langley DR, and Krystal M. Changes to the HIV Long Terminal repeat and to HIV Integrase Differentially Impact HIV Integrase Assembly, Activity, and the Binding of Strand Transfer Inhibitors. Journal of Biological Chemistry. 2007. 43: 31186-1196

Dowling WE, Kim B, Mason CJ, Birx DL, Robb ML, McCutchan FE, and Carr JK. Forty-one near full-length HIV-1 sequences from Kenya reveal an 
epidemic of subtype A and A-containing recombinants. AIDS. 2002. 16: 18091820

Drummond AJ and Rambaut A. BEAST: Bayesian evolutionary analysis by sampling trees. BioMed Central Evolutionary Biology. 2007. 7; 214

Engelbrecht S, de Villiers T, Sampson CC, zur Megede J, Barnett SW, and Janse van Rensburg E. Genetic Analysis of the Complete gag and env Genes of HIV Type 1 Subtype C Primary Isolates from South Africa. AIDS Research and Human Retroviruses. 2001. 17: 1533-1547

Engelbrecht S, Laten JD, Smith T-L, and van Rensburg EJ. Identification of env subtypes in fourteen HIV type 1 isolates from South Africa. AIDS Research and Human Retroviruses. 1995. 11: 1269-1271

Engelbrecht S, Smith T-L, Kasper P, Faatz E, Zeier M, Moodley D, Clay CG, and van Rensburg EJ. HIV Type 1 V3 Domain Serotyping and Genotyping in Gauteng, Mpumalanga, KwaZulu-Natal, and the Western Cape Provinces of South Africa. AIDS Research and Human Retroviruses. 1999. 15: 325-328

Engelbrecht S, de Jager GJ, and van Rensburg EJ. Evaluation of commercially available assays fro antibodies to HIV-1 in serum obtained from South African patients infected with HIV-1 subtypes B, C, and D. Journal of Medical Virology. 1994. 44: 223-228

Esparza $\mathrm{J}$ and Bhamarapravati $\mathrm{N}$. Accelerating the development and future availability of HIV-1 vaccines: why, when, where and how? Lancet. 2000. 355; 2061-2066

Essex M, Hardy WD Jr, Cotter SM, Jakowski RM, and Sliski A. Naturally occurring persistent feline oncornavirus infections in the absence of disease. Infection and Immunity. 1975.11(3): 470-475

Essex M and Kanki PJ. The origins of the AIDS virus. Science America. 1998. 259: 64-71 
Essex $M$ and Mboup S. AIDS in Africa (2 ${ }^{\text {nd }}$ edition). Edited by Essex $M$, Mboup S, Kanki PJ, Marlink RG and Tlou SD. Kluwer Academic/Plenum Publishers, New York (NY), United States of America. 2002.

Fahey JL, Prince H, Weaver M, Groopman J, Visscher B, Schwartz K, and Detels R. Quantitative changes in $T$ helper or $T$ suppressor/cytotoxic lymphocyte subsets that distinguish acquired immune deficiency syndrome from other immune subset disorders. American Journal of Medicine. 1984. 76: $95-100$

Fang G, Weiser B, Visosky AA, Townsend L, and Burger H. Molecular Cloning of Full-length HIV-1 Genomes Directly from Plasma Viral RNA. AIDS and Human Retroviruses. 1996. 12: 352-357

Felsenstein J. Numerical methods for inferring evolutionary trees. Quarterly Review of Biology. 1982. 57: 379-404.

Felsenstein J. Confidence limits on phylogenies: An approach using the bootstrap. Evolution. 1985. 39: 783-791

Fischetti L, Opare-Serri O, Candotti D, Sarkodie F, Lee H, and Allaini JP. Molecular Epidemiology of HIV in Ghana: Dominance of CRF02_AG. Journal of Medical Virology. 2004. 73: 158-166

Fitch WM and Margoliash E. Construction of phylogenetic trees. Science. 1967. 20: 279-284

Flügel RM. Spumaviruses: a group of complex retroviruses. AIDS. 1991. 4: $739-750$

Friedman-Kien AE, Laubenstain L, Marmor M, Hymes K, Green J, Ragaz A, Gottleib J, Muggia F, Demopoulos R, Weintraub M, Williams D, Oliveri R, Marmer J, Wallace J, Halperin I, Gillooley JF, Prose N, Klein E, Vogel J, Safai B, Myskowski P, Urmacher C, Koziner B, Nisce L, Kris M, Armstrong D, Gold J, Mildran D, Tapper M, Weissman JB, Rothenberg R, Friedman SM, Siegal FP, Groundwater J, Gilmore J, Coleman D, Follansbee S, Gullett J, Stegman 
SJ, Wofsy C, Bush D, Drew L, Braff E, Dritz S, Klein M, Preiksaitis JK, Gottlieb MS, Jung R, Chin Jand Goedert J. Kaposi's sarcoma and Pneumocystis pneumonia among homosexual men - New York City and California. 1981. Morbitity and Mortality Weekly Report - Center for Disease Control and Prevention. 30: 305-308

Gallo RC. The discovery of the first human retrovirus: HTLV-1 and HTLV-2. Retrovirology. 2005. 2: 17

Gallo RC, Salahuddin SZ, Popvic M, Shearer GM, Kaplan M, Haynes BF, Palker TJ, Redfield R, Olseke J, and Safai B. Frequenct detection and isolation of cytopathic retroviruses (HTLV-III) from patients with AIDS and at risk of AIDS. Science. 1984. 224(4648): 500-503

Gange RW, and Jones EW. Kaposi's sarcoma and immunosuppressive therapy: an appraisel. Clinical Experimental Dermatology. 1978. 3: 135-146

Gao F, Bailes E, Robertson DL, Chen Y, Rodenburg CM, Michael SF, Cummins LB, Arthur LO, Peeters M, Shaw GM, Sharp PM, and Hahn BH. Origin of HIV-1 in the chimpanzee Pan troglodytes troglodytes. Nature. 1999. 97: 436-441.

Gao F, Robertson DL, Morrison SG, et al. The heterosexual human immunodeficiency virus type 1 epidemic in Thailand is caused by an intersubtype (A/E) recombinant of African origin. Journal of Virology. 1996. 70: 7013-7029

Gao F, Yue L, and White AT. Human infection by genetically diverse SIVsm related to HIV-2 in West Africa. Nature. 1992. 358: 495-499

Gao F, Yue L, Robertson DL, Hill SC, Hui H, Biggar RJ, Neequaye AE, Whelan TM, Ho DD, Shaw GM, Sharp PM, and Hahn BH. Genetic diversity of Human Immunodeficiency Virus Type2: Evidence for Distinct Sequence Subtypes with Differences in Virus Biology. Journal of Virology. 1994. 68: 7433-7447 
Gelderblom H (1997).Fine Structure of HIV and SIV. pp. IV-37-50 in HIV Molecular Immunology Database 1997. Edited by: Korber B, Brander C, Haynes B, Koup R, Moore J, Walker B. Published by: Theoretical Biology and Biophysics Group, Los Alamos National Laboratory, Los Alamos, NM. 2007

Geretti AM. HIV-1 subtypes: epidemiology and significance of HIV management. Current Opinions in Infectious Diseases. 2006. 19: 1-7

Goldberg B, and Stricker RB. Apoptosis and HIV infection: T-cells fiddle while the immune system burns. Immunology Letters. 1999. 70: 5-8

Goldman N and Yang Z. A codon-based model of nucleotide substitution for protein-coding DNA sequences. Molecular Biological Evolution. 1994. 11: 725-736

Gordon M, de Oliveira T, Bishop K, Coovadia HM, Madurai L, Engelbrecht S, Janse van Rensburg E, Mosam A, Smith A, and Cassol S. Molecular Characteristics of Human Immunodeficiency Virus Type 1 Subtype C Viruses from KwaZulu-Natal, South Africa: Implications for Vaccine and Antiretroviral Control Strategies. Journal of Virology. 2003. 77: 2587-2599

Gottlieb MS, Schroff R, Schanker HM, Weisman JD, Fan PT, Wolf RA and Saxon A. Pneumocystis carinii pneumonia and mucosal candidiasis in previously healthy homosexual men: Evidence of a new acquired cellular immune deficiency. New England Journal of Medicine. 1981. 305: 1425-1428

Graur D and Li WH. Fundamentals of molecular evolution. Sinauer Associates, Sunderland, MA, USA. 1999.

Guindon S, and Gascuel O. A simple, fast and accurate algorithm to estimate larges phylogenies by maximum likelihood. Syst. Biol. 2003. 696-704

Gurtler LG, Hauser PH, Eberle J, von Brunn A, Knapp S, Zekeng L, Tsague JM, and Kaptue L. A new subtype of human immunodeficiency virus type 1 (MVP-5180) from Cameroon. Journal of Virology. 1994. 68: 1581-1585 
Hahn BH, Shaw GM, De Cock KM, and Sharp PM. AIDS as a Zoonosis: Scientific and public health implications. Science. 2000. 287: 607-614_

Hall BG. Phylogenetic Trees Made Easy: A How-To Manual. Sunderland Associates, Inc. Massachusetts, United States of America. 2004

Hall T. BioEdit, Biological sequence alignment for Windows 95/98/NT. 2001

Hardy WD Jr, Old LJ, Hess PW, Essex M, and Cotter S. Horizontal transmission of feline leukaemia virus. Nature. 1973. 244: 266-269

Hasegawa M, Kishino $\mathrm{K}$, and Yano T. Dating the human-ape splitting by a molecular clock of mitochondrial DNA. Journal of Molecular Evolution. 1985. 22: $160-174$

Hasegawa A, Tsujimoto H, Maki N, Ishikawa KI, Miura T, Fukasawa M, Miki K, and Hayami M. Genomic divergence of HIV-2 from Ghana. AIDS Research Human Retroviruses. 1989. 5: 593-604

Hayman A, Moss T, Simmons G, Arnold C, Holmes EC, Naylor-Adamson L, Hawkswell J, Allen K, Radford J, Nguyen-Van-Tam J and Balfe P. 2001.

Phylogenetic analyses of multiple heterosexual transmission events involving 10 subtype B of HIV type 1. Aids Research and Human Retroviruses. 17: 689695.

Hemelaar J, Gouws E, Ghys PD, and Osmanov S. Global and regional distribution of HIV-1 genetic subtypes and recombinants in 2004. AIDS. 2006. 20(16): W13 - W23

Hirsch V, Olmsted R, Murphy-Corb M, Pyrcell A and Johnson P. An African primate lentivirus (SIVsm) closely related to HIV-2. Nature. 339: 389-392

Ho DD, Neumann AU, Perelson SA, Chen J, Leonard JM, and Markowits M. Rapid turnover of plasma virions and CD4 lymphocytes in HIV-1 infections. Nature. 1995. 373: 123-126 
Hogeweg $\mathrm{P}$ and Hesper $\mathrm{B}$. The alignment of sets of sequences and the construction of phylogenetic trees: An integrated method. Journal of Molecular Evolution. 1984. 20: 175-186

Holmes I. Sequence homology search tools on the World Wide Web. pp. 4453 in HIV Sequence Compendium 2000. Edited by: Kuiken C, McCutchan F, Foley B, Mellors JW, Hahn B, Mullins J, Marx P, Wolinsky S. Published by: Theoretical Biology and Biophysics Group, Los Alamos National Laboratory, Los Alamos, NM. 2000.

Howard TM, and Rasheed S. Genomic structure and nucleotide sequence analysis of a new HIV type 1 subtype A strain from Nigeria. AIDS Research and Human Retroviruses. 1996. 12: 1413-1425

\section{http://en.wikipedia.org/wiki/HIV}

http://www.hiv.lanl.gov/ - HIV Sequence Compendium, 06/07. Leitner T, McCutchan F, Foley B, Mellors JW, Hahn B, Wolinsky S, Marx P and Korber B. Theoretical Biology and Biophysics Group, Los Alamos National Laboratory, Los Alamos, NM, LA-UR 03-3564

\section{http://www.talkorigins.org/}

Huebner RJ, and Todaro GJ. Oncogenes of RNA tumor viruses as determinants of cancer. Proceedings of the National Academy of Science USA. 1969. 64: 1087-1094

Hunt GM, Johnson D, and Tiemessen CT. Characterisation of the Long Terminal Repeat Regions of South African Human Immunodeficiency Virus Type 1 Isolates. Virus Genes. 2001. 23: 27-34

Hunt GM, Papathanasopoulos MA, Gray GE, and Tiemessen CT. Characterization of Near-full Length Genome Sequences of Three South African Human Immunodeficiency Virus Type 1 Subtype C Isolates. Virus Genes. 2003. 26: 49-56 
Hymes KB, Cheung T, Greene JB, Prose NS, Marcus A, Ballard H, and Williams DC. 'Kaposi's sarcoma in homosexual men: A report of eight cases', Lancet. 1981. 2: 598-600

Jacobs GB, de Beer C, Fincham JE, Adams V, Dansay MA, Janse Van Rensburg E, and Engelbrecht S. Serotyping and Genotyping of HIV-1 Infection in Residents of Khayelitsha, Cape Town, South Africa. Journal of Medical Virology. 2006. 78: 1529-1536

Jacobs GB, Loxton AG, Laten A, and Engelbrecht S. Complete Genome Sequencing of a Non-syncytium-Inducting HIV Type 1 Subtype D Strain from Cape Town, South Africa. AIDS Research and Human Retroviruses. 2007. 23: 1575-1578

Jacobs GB, Nistal M, Laten A, Janse van Rensburg E, Rethwilm A, Preiser W, Bodem J, and Engelbrecht S. Molecular Analysis of HIV Type 1 vif Sequences from Cape Town, South Africa. AIDS Research and Human Retroviruses. 2008a. 24: (991-994)

Jacobs GB, Loxton AG, Laten JD, Brenda R, van Rensburg EJ, and Engelbrecht S. Emergence and diversity of different HIV-1 subtypes in Cape Town, South Africa. Journal of Medical Virology. Submitted

Jacobs GB, Laten AD, van Rensburg EJ, Bodem J, Weissbrich B, Rethwilm A, Preiser W, and Engelbrecht S. Phylogenetic Diversity and Low Level Antiretroviral Resistance Mutations in HIV Type 1 Treatment-Naive Patients from Cape Town, South Africa. AIDS Research and Human Retroviruses. 2008b. 24: (1009-1012)

Jaffe HW, Francis DP, McLane MF, Cabradilla C, Curran JW, Kilbourne BW, Lawrence DN, Haverkos HW, Spira TJ, and Dodd RY. Transfusion-associated AIDS: serologic evidence of human T-cell leukemia virus infection of donors. Science. 1984. 223: 1309-1312

Janssens W. Heyndrickx L, Fransen K, Motte J, Peeters M, Nkengasong JN, Ndumbe PM, Delaporte E, Perret JL, Atende C, Piot P, and van der Groen G. 
Gentic and phylogentic analysis of env subtypes $G$ and $H$ in Central Africa. AIDS Research and Human Retroviruses. 1994. 10: 877-879

Jukes T, and Cantor CR. Evolution of protein molecules. In: Mammalian protein Metabolism, edited by Munro HN, pp 21-132. New York, Academic Press (1969).

Kanki PJ, Travers KU, Mboup S, Hsieh CC, Marlink RG, and Gueye-NDiaye A. Slower heterosexual spread of HIV-2 than HIV-1. Lancet. 1994. 343: 943946

Kemp DJ, Smith DB, Foote SJ, Samaras N, and Petersen MG. Colorimetric detection of specific DNA segments amplified by polymerase chain reactions. Proceedings of the National Academy of Sciences. 1989. 86: 2423-2427.

Kimura M. The Neutral Theory of Molecular Evolution. Cambridge: Cambridge University Press, UK. 1980

Korber B, Muldoon M, Theiler J, Gao F, Gupta R, Lapedes A, Hahn BH, Wolinsky S, and Bhuttacharga T. Timing the ancestor of the HIV-1 pandemic strains. Science. 2000. 288: 1789-1795

Lara C, Sällberg M, Johasen B, De Rivera I and Sönnerborg A. 1 1997. The Honduran human immunodeficiency virus type 1 (HIV-1) epidemic is dominated by HIV-1 subtype $B$ determined by V3 domain sero and genotyping. Journal of Clinical Microbiology 35: 783-784.

Learn GH, Korber BTM, Foley B, Hahn BH, Wolinsky SM, and Mullins JI. Maintaining the integrity of human immunodeficiency virus database. 1996. Journal of Virology. 70: 5720-5730

Leitner T, Foley B, Hahn B, et al. HIV sequence compendium 2005. Los Alamos, NM: Theoretical Biology and Biophysics Group, Los Alamos National Laboratory, 2005. 
Leitner T. Genetic subtypes of HIV-1. In Human Retroviruses and AIDS 1996. A compilation and analysis of nucleic acid and amino acid sequences. Myers G, Korber BT, Foley BT. Theoretical Biology and Biophysics Group. Los Alamos National Laboratory, Los Alamos, New Mexico. 1996

Lemey P, Pybus OG, Rambaut A, Drummond AJ, Robertson DL, Roques P, Worobey M, and Vandamme A-M. The Molecular population Genetics of HIV1 Group O. Genetics. 2004. 167: 1059-1068

Lemey P, Pybus OG, Wang B, Saksena NK, Salemi M, and Vandamme A-M. Tracing the origin and history of the HIV-2 epidemic. Proceedings of the National Academy of Science. 2003. 100(11): 6588-6952

Levy JA, Hoffman AD, Kramer SM, Landis JA, Shimabukuro JM, and Oshiro LS. Isolation of lymphocytopathic retroviruses from San Francisco patients with AIDS. Science. 1984. 225: 840-842

Levy JA. HIV and the Pathogenesis of AIDS. $3^{\text {rd }}$ Edition. ASM Press, Washington D.C. (2007).

Li WH. Molecular evolution. Sinauer Associates, Sunderland, Massachusetts (MA), USA. 1997

Lole KS, Bollinger RC, Paranjape RS, Gadkari D, Kulkarni SS, Novak NG, Ingersoll R, Sheppard HW, Ray SC. Full-length human immunodeficiency virus type 1 genomes from subtype C-infected seroconverters in India, with evidence of intersubtype recombination. Journal of Virology. 1999. 73: 152160

Louwagie J, McCutchan FE, Peeters M, Brenamn TP, Sanders-Buell E, Eddy GA, van der Groen G, Fransen K, Gershy-Damet GM, Deleys R, and Burke DS. Phylogenetic analysis of gag genes from 70 international HIV-1 isolates provides evidence for multiple genotypes. AIDS. 1993. 7: 769-780

Loxton AG, Treurnicht F, Laten A, Janse Van Rensburg E, and Engelbrecht S. Sequence analysis of near full-length HIV type 1 subtype $D$ primary strains 
isolated in Cape Town, South Africa, from 1984 to 1986. AIDS Research and Human Retroviruses. 2005. 21: 410-413

Markovitz D. Infection with the human immunodeficiency virus type 2. Annuals of Internal Medicine. 1993. 188: 211-218

Marlink R, Kanki PJ, Thior I, Travers K, Eisen G, Siby T, Traore I, Hsieh CC, Dia MC, and Gueye EH. Reduced rate of disease development after HIV-2 infection as compared to HIV-1. Science. 1994. 265: 1587-1590

Martin DP, Williamson C, and Posada D. RDP2: recombination detection and analysis from sequence alignments. Bioinformatics. 2004. 21: 260-262

Masur H, Michelis MA, Greene JB, Onorato I, Stouwe RA, Holzman RS, Wormser G, Brettman L, Lange M, Murray HW, and Cunningham-Rundles S. An outbreak of community-acquired Pneumocystis carinii pneumonia: initial manifestation of cellular immune dysfunction. New England Journal of Medicine. 1981. 305: 1431-1438

Mau B, Newton MA and Larget B. Bayesian phylogenetic inference via Markov chain Monte Carlo methods. Biometrics. 1999. 55: 1-12

McCormack GP and Clewley JP. The application of molecular phylogenetics to the analysis of viral genome diversity and evolution. Review in Medical Virology. 2002. 12: 221-238

McCutchan FE, Carr JK, Bajani M, Sanders-Buell E, Harry TO, Stoeckli TC, Robbins KE, Gashau W, Nasidi A, Janssens W, and Kalish ML. Subtype G and multiple forms of $A / G$ intersubtype recombinant human immunodeficiency virus type 1 in Nigeria. Virology. 1999. 254: 226-234

McCutchan FE. Global epidemiology of HIV. Journal of Medical Virology. 2006. 78(suppl. 1): S7-S12

McCutchan FE, Hegerich PA, Brennan TP, et al. Genetic variants of HIV-1 in Thailand. AIDS Research Human Retroviruses. 1992. 8: 1887-1895 
McCutchan FE, Salminen MO, Carr JK, and Burke DS. HIV-1 genetic diversity. AIDS. 1996. 10(Suppl. 3): S13-S20

Menu E, Truong TX, Lafon ME, Nguyen TH, Muller-Trutwin MC, Nguyen TT, Deslandres A, Chaouat G, Duong QT, Ha BK, Fleury HJ, and Barre-Sinoussi F. HIV type 1 Thai subtype $E$ is predominant in South Vietnam. AIDS Research and Human Retroviruses. 1996. 12: 629-633

Michael NL. Host genetic influences on HIV-1 pathogenesis. Current Opinions in Immunology. 1999. 11: 466-474

Mwaengo DM, and Novembre FJ. Molecular cloning and characterization of viruses isolated from chimpanzees with pathogenic human immunodeficiency virus type 1 infections. Journal of Virology. 1998. 72: 8976-8987

Myers G. HIV-1 and HIV-2 sequence subtypes. Human Retroviruses and AIDS. 1992. (Myers G, Korber B, Berzofsky JA, and Smith RA., eds) Los Alamos National Laboratory, Los Alamos, NM. 1992.

Myers G, Maclnnes K, and Korber B, The emergence of simian/human immunodeficiency viruses. AIDS Research Human Retroviruses. 1992. 8: 373-386

Myers G. Tenth anniversary perspectives on AIDS. HIV: between the past and future. AIDS Research and Human Retroviruses. 1994. 10: 1317-1324

Nadai Y, Eyzaguirre ML, Constantine NT, Sill AM, Cleghorn F, Blattner WA, and Carr JK. Protocol for Nearly Full-Length Sequencing of HIV-1 RNA from Plasma. PLOS one. 2008. 1: e1420

Nahmias AJ, Weiss J, Yao X, Lee F, Kodsi R, Schanfield M, Matthews T, Bolognesi D, Durack D, and Motusky A. Evidence for human infection with an HTLV-III/LAV like virus in central Africa, 1952. Lancet. 1986. I: 373-386

Njai HF, Gali Y, Vanham G, Clybergh C, Jennes W, Vidal N, Butel C, MpoudiNgolle E, Peeters M, and Ariên KK. The predominance of Human 
Immunodeficiency Virus type 1 (HIV-1) circulating recombinant form 02 (CRF02_AG) in West Central Africa may be related to its replicative fitness. Retrovirology. 2006. 3:40 4690-4701

Olseke J, Minnefor A, and Cooper R Jr. Immune deficiency syndrome in children. Journal of American Medical Association. 1983. 249: 2345-2349

Op de Coul ELM, Prins M, Cornelissen M, van der Schoot A, Boufassa F, Brettle RP, Hernandez-Aguado I, Schiffer V, McMenamin J, Rezza G, Robertson R, Zangerie R, Goudsmit J, Coutinho RA, and Lukashov V. Using phylogenetic analysis to trace HIV-1 migration among western European infecting drug users seroconverting from 1984 to 1997. AIDS. 2001. 15: 257266

Osmanov S, Pattou C, Walker N, Schwarlander B, Esparza J and the WHOAIDS Network for HIV isolation and Characterisation. Estimated global distribution and regional spread of HIV-1 genetic subtypes in the year 2002. Journal of Acquired Immune Deficiency Syndrome. 2002. 29: 184-190

Ou C-Y, Kwok S, Mitchell SW, Mach DH, Sninsky JJ, Krebs JW et al,. DNA amplification for direct detection of HIV-1 in DNA of peripheral blood mononuclear cells. Science. 1988. 239: 295-297

Page RDM and Holmes EC. Molecular evolution: A phylogenetic approach. Blackwell Science Ltd. Oxford, United Kingdom. 1998.

Papathanasopoulos MA, Cilliers T, Morris L, Mokili JL, Dowling W, Brix DL, and McCutchan FE. Full-Length Genome Analysis of HIV-1 Subtype C Utilizing CXCR4 and Intersubtype Recombinants Isolated in South Africa. AIDS Research and Human Retroviruses. 2002. 18: 879-886

Papathanasopoulos MA, Cilliers T, Morris L, Mokili JL, Dowling W, Carr JK, McCutchan F. First Full-Genome Sequences of Intersubtype Recombinants Circulating in South Africa. AIDS Vaccine 2001, Sep 5-8; National Institute of Virology, Johannesburg, South Africa. 2001. 
Papathanasopoulos MA, Hunt GM, and Tiemessen CT. Evolution and Diversity of HIV in Africa - a Review. Virus Genes. 2003a. 26: 151-163

Papathanasopoulos MA, Patience T, Meyers TM, McCutchan FE, and Morris L. Full-Length Genome Characterization of HIV Type 1 Subtype C Isolates from Two Slow-Progressing Perinatally Infected Siblings in South Africa. AIDS Research and Human Retroviruses. 2003b. 19: 1033-1037

Peeters M. Recombinant HIV sequences: Their role in the global epidemic. pp. I-39-54 in HIV Sequence Compendium. 2000. Edited by: Kuiken CL, and Foley $\mathrm{B}$.

Peeters M. The genetic variability of HIV-1 and its implications. Tansfus Clin Biol. 2001. 8: 222-225.

Peeters M, Toure-Kane C, and Nkengasong JN. Genetic diversity of HIV in Africa: impact on diagnosis, treatment, vaccine development and trails [EDITORIAL REVIEW]. AIDS. 2003. 17: 2547-256

Penn I. Kaposi's sarcoma in organ transplant recipients: report of 20 cases. Transplantation. 1979. 27: 8-11

Pepin J, Morgan G, Dunn D, Gevao S, Mendy M, Gaye I, Scollen N, Tedder $\mathrm{R}$, and Whttle $\mathrm{H}$. HIV-2-induced immunosuppression among asymptomatic West African prostitutes: evidence that HIV-2 is pathogenic, but less so than HIV-1. AIDS. 1991. 5: 1165-1172

Pieniazek D, Yang C, and Lal RB. Phylogenetic Analysis of gp41 Envelope of HIV-1 Groups M, N and $\mathrm{O}$ in Human Retroviruses and AIDS 1998: A Compilation and Analysis of Nucleic Acid and Amino Acid Sequences. Korber B, Kuiken CL, Foley B, Hahn B, McCutchan F, Mellors JW, and Sodroski J, eds. Theoretical Biology and Biophysics Group, Los Alamos National Laboratory, Los Alamos, NM. 1998. 
Plantier JC, Dachraoui R, Lemee V, Gueudin M, Borsa-Lebas F, Caron F, and Simon F. HIV-1 resistance genotyping on dried serum spots. AIDS. 2005. 19(4): 391-397

Potts KE, Kalish ML, Bandea Cl, Orloff GM, St. Louis M, Brown C, Malanda N, Kavuka M, Schochetman G, Ou CY, and Heyward WL. Genetic diversity of human immunodeficiency virus type 1 strain in Kinshasa, Zaire. AIDS Research Human Retroviruses. 1993. 9: 613-618

Quinn TC, Mann JM, Curran JW, and Piot P. AIDS in Africa: An Epidemiologic Paradigm. Science. 1986. 234: 955-963

Rannala B and Yang Z. Probability distribution of molecular evolutionary trees: a new method of phylogenetic inference. Journal of Molecular Evolution. 1996. 43: 304-311

Ras GJ, Simson IW, Anderson R, Prozeksy OW and Hamersma T. Acquired immunodeficiency syndrome: a report of 2 South African cases. South African Medical Journal. 1983. 64: 140-142

Ratner L, Haseltine W, Patarca R, Livak KJ, Starcich B, Josephs SF, Doran ER, Rafalski A, Whitehorn EA, Baumeister K, Ivanoff L, Petteway SR, Pearson ML, Lauenberger JA, Papas TS, Ghrayeb J, Chang NT, Callo RC and Wong-Staal F. Complete nucleotide sequence of the AIDS virus, HTLVIII. Nature 1985a. 313: 227-284

Ratner L, Gallo RC, and Wong-Staal F. HTLV-III, LAV, ARV are variants of same AIDS virus. Nature. 1985b 313: 636-637

Rey-Cuille MA, Berthier JL, Bomsel-Demontoy MC, Chaduc Y, Montagnier L, Hovanessian LA, and Chakrabarati LA. Simian immunodeficiency virus replicates to high levels in sooty Mangabeys without inducing disease. Journal of Virology. 1998. 72: 3872-3886

Robertson DL, Anderson JP, Bradac JA, Carr JK, Foley B, Funkhouser RK, Gao F, Hahn BH, Kalish ML, Kuiken C, Learn GH, Leither T, McCutchan F, 
Osmanov S, Peeters M, Pieniazek D, Salminen M, Sharp PM, Wolinsky S, and Korber B. HIV-1 nomenclature proposal. Science. 2000. 288: 55-56

Roberts JD, Bebenek K, and Kunkel TA. The accuracy of reverse transcriptase from HIV-1. Science. 1988. 242: 1171-1173

Robertson DL, Sharp PM, McCutchan FE, ad Hahn BH. Recombination in HIV-1. Nature. 1995. 374: 124-126

Rodenburg CM, Li Y, Trask SA, Chen Y, Decker J, Robertson DL, Kalish ML, Shaw GM, Allen S, Hahn BH, Gao F, UNAIDS and NIAID Networks for HIV Isolation and Characterization. Near full-length clones and reference sequences for subtype $\mathrm{C}$ isolates of HIV type 1 from three different continents. AIDS Research and Human Retroviruses. 2001. 17: 161-168

Rodriguez F, Oliver JF, Marin A, and Medina JR. The general stochastic model of nucleotide substitution. Journal of Theoretical Biology. 1990. 142: 485-501

Rodrigo AG and Learn GH Jr. Computational and Evolutionary analysis of HIV molecular sequences. Kluwer Academic Publishers. Dordrecht, The Netherlands. 2001.

Rousseau CM, Birditt BA, McKay AR, Stoddard JN, Lee TC, McLauglin S, Moore SW, Shindo N, Learn GH, Korber BT, Brander C, Goulder PJR, Kiepiela P, Walker BD, and Mullins Jl. Large-scale amplification, cloning and sequencing of near full-length HIV-1 subtype $C$ genomes. Journal of Virological Methods. 2006. 136: 118-125

Rubinstein A, Sticklick M, Gupta A, Bernstein L, Klein N, Rubinstein E, Spigland I, Fruchter L, Litman N, Lee H, and Hollander M. Acquired immunodeficiency with reversed T4/T8 ratios in infants born to promiscuous and drug-addicted mothers. Journal of American Medical Assosiation. 1983. 249: $2350-2356$ 
Saitou $\mathrm{N}$ and Nei M. The neighbour-joining method: a new method for reconstructing phylogenetic trees. Molecular Biology Evolution. 1987. 4: 406425

Salminen MO, Koch C, Sanders-Buell E, Ehrenberg PK, Michael NL, Carr JK, Burke DS, and McCutchan FE. Recovery of virtually full-length HIV-1 provirus of diverse subtypes from primary virus cultures using the polymerase chain reaction. Virology. 1995a. 213: 80-86

Salminen MO, Carr JK, Burke DS, and McCutchan FE. Identification of breakpoints in intergenotypic recombinats of HIV type 1 by booscanning. AIDS Research and Human Retroviruses. 1995b. 11: 1423-1425

Salemi M and Vandamme A-M. The Phylogenetic Handbook: A practical approach to DNA and protein phylogeny. Cambridge University Press. Cambridge, United Kingdom. 2003

Salemi M, Strimmer K, Hall WW, Duffy M, Delaporte E, Mboup S, Peeters M and Vandamme A-M. Dating the common ancestor of SIVcpz and HIV-1 group $M$ and the origin of HIV-1 subtypes using a new method to uncover clock-like molecular evolution. The FASEB Journal. 2001. 15: 276-278

Sanders-Buell E, Salminen MO, and McCutchan FE. Sequencing primers for HIV-1 IN: Human Retroviruses and AIDS 1995: Kuiken C, Foley B, Hahn B, Marx P, McCutchan F, Mellors J, Wolinsky S, and Korber B. A compilation and analysis on nucleic acid and amino acid sequences. Theoretical Biology and Biophysics Group, Los Alamos National Laboratory, Los Alamos, NM, USA. 1995.

Schultz AK, Zhang M, Leitner T, Kuiken C, Korber B, Morgenstern B, and Stanke M. A jumping profile Hidden Markov Model and applications to recombination sites in HIV and HCV genomes. BMC Bioinformatics. 2006. 7: 265-280

Scriba TJ, de Villiers T, Treurnicht FK, zur Megede J, Barnett SW, Engelbrecht S, and van Rensburg EJ. Characterization of the South African 
HIV Type 1 C Complete 5' Long Terminal Repeat, nef, and Regulatory genes. AIDS Research and Human Retroviruses. 2002. 18: 149-159

Serwadda D, Mugerwa RD, Sewankambo NK, Lwegaba A, Carswell JW, Kirya GB, Bayley AC, Downing RG, Tedder RS, and Clayden SA. 1985. Slim disease: a new disease in Uganda and its association with HTLV-III infection. Lancet. 2: 849-852

Shankarappa R, Margolick JB, Gange SJ, Rodrigo AG, Upchurch D, Fazadegan H, Gupta P, Rinaldo CR, Learn GH, He XL and Mullins JI. Consistent viral evolutionary dynamics associated with the progression of HIV-1 infection. Journal of Virology. 1999. 73: 10489-10502

Sher R. HIV infection in South Africa, 1982 - 1988 - a review. South African Medical Journal. 1989. 76(Oct): 314-318

Shilts RM. And the band Played on: Politics, people and the AIDS epidemic. St. Martin's Press. New York, USA. 1987.

Siepel AC, Halpern AL, Macken C, and Korber BT. A computer program designed to screen rapidly for HIV type 1 intersubtype recombinant sequences. AIDS Research Human Retroviruses. 1995. 11: 1413-1416

Silvestri G, Sodora D, Koup R, Paiardini M, O'Neil S, McClure S, Staprans S, and Feinberg M. Nonpathogenic SIV infection of Sooty Mangabeys is characterized by limited bystander immunopathology despite chronic highlevel viremia. Immunity. 2003. 18: 441-452

Sneath PHA and Sokal RR. Numerical Taxonomy. Freeman, San Francisco, USA. 1973.

Songok EM, Lwembe RM, Kibaya R, Kobayashi K, Ndembi N, Kita K, Vulule J, Oishi I, Okoth F, Kageyama S, and Icimura H. Active Generation and Selection of HIV Intersubtype A/D Recombinant Forms in a Coinfected Patient in Kenya. AIDS Research and Human Retroviruses. 2004. 20: 255-258 
Spang R, Rehmsmeier M, and Stoye J. A Novel Approach to Remote Homology Detection: Jumping Alignments. Journal of Computational Biology. 2002. 9: $747-760$

Spira S, Wainberg MA, Loemba H, Turner D, and Brenner BG. Impact of clade diversity of HIV-1 virulence, antiretroviral drug sensitivity and drug resistance. Journal of Antimicrobial Chemotherapy. 2003. 51: 229-240

Swanson P, Devare SG, and Hackettt J Jr. Molecular Characterization of 39 HIV-1 Isolates Representing Group M (Subtype A-G) and Group O: Sequence Analysis of gag p24, pol Integrase, and env gp41. AIDS Research and Human Retroviruses. 2003. 19: 625-629

Swofford DL. PAUP*. Phylogenetic Analysis Using Parsimony (* and other methods). Version 4.0b10. Sunderland, Massachusetts (USA): Sinauer Associates, Inc. 2002.

Tamura K, Dudley J, Nei M, and Kumar S. MEGA4: Molecular Evolutionary Genetics Analysis (MEGA) software version 4.0. Molecular Biology and Evolution. 2007. 24:1596-1599.

Tavaré S. Some Probabilistic and Statistical Problems in the Analysis of DNA Sequences. American Mathematical Society: Lectures on Mathematics in the Life Sciences. 1986. 17: 57-86.

Taylor BS, Sobieszcczyk ME, McCutchan FE, and Hammer SM. The Challenge of HIV-1 Subtype Diversity. New England Journal of Medicine. 2008. 358: 1590-1602

Temin HM. Retrovirus variation and reverse transcription: abnormal strand transfers result in retrovirus genetic variation. Proc. Natl. Acad. Sci USA. 1993. 22: 6900-6903

Thompson JD, Higgins DG and Gibson TJ. CLUSTAL W: Improving the sensitivity of progressive multiple-sequence alignment through sequence 
weighting, position-specific gap penalties, and weight matrix choice. Nucleic Acids Research. 1994. 22: 4673-4680

Thompson JD, Gibson TJ, Plewniak F, Jeanmougin F and Higgins DG. The CLUSTAL $X$ windows interface: Flexible strategies for multiple-sequence alignment aided by quality analysis tools. Nucleic Acids Research. 1997. 25: 4876-4882

Torques K, Bourgeois A, Saragosti S, Vidal N, Mpoudi-Ngolle E, Nzilambi N, Apetrei C, Ekwalanga M, Delaporte E, and Peeters M. High diversity of HIV-1 subtype F strains in Central Africa. Virology. 1999. 259: 99-109

Treurnicht FK, Smith T-L, Engelbrecht S, Claassen M, Robson BA, Zeier M, and van Rensburg EJ. Genotypic and Phenotypic Analysis of the Env Gene From South African HIV-1 Subtype B and C Isolates. Journal of Medical Virology. 2002. 68: 141-146

UNAIDS 2008. 2008 Report on the Global AIDS epidemic. http://www.unaids.org/en/

Uchiyama T, Yodoi J, Sagawa K, Takatsuki K, and Uchino H. Adult T-cell leukemia: clinical and hematologic features of 16 cases. 1977. Blood. 50: 481492

Vandamme A-M, Salemi M, van Brussel M, Liu HF, Van Laethem K, Van Ranst M, Michels L, Desmyter J, and Boubau P. African Origin of Human TLymphotropic Virus Type 2 (HTLV-2) Supported by a Potential New HTLV-2d Subtype in Congolese Bambuti Efe Pygmies. Journal of Virology. 1998. 72: 4327-4340

van Harmelen JH, van der Ryst E, Loubser AS, York D, Madurai S, Lyons S, Wood R, and Williamson C. A Predominantly HIV-1 Subtype C-Restricted Epidemic in South African urban Populations. AIDS Research and Human Retroviruses. 1999. 15: 395-398 
van Harmelen J, Wood R, Lambrick M, Rybicki EP, Williamson AL, and Williamson C. An association between HIV-1 subtypes and mode of transmission in Cape Town, South Africa. AIDS. 1997. 11: 81-87

van Harmelen J, Williamson C, Kim B, Morris L, Carr J, Karim SS, and McCutchan $F$. Characterization of full-length HIV type 1 subtype $C$ sequences from South Africa. AIDS Research and Human Retroviruses. 2001. 17: 15271531

van Rossum AMC, Fraaii PLA, and de Groot R. Efficacy of highly active antiretroviral therapy in HIV-1 infected children. The lancet Infectious Diseases. 2002. 2: 93-102

Vidal N, Peeters M, Mulanga-Kabeya C, Nzilambi N, Robertson D, llunga W, Sema H, Tshimanga K, Bongo B, and Delaporte E. Unprecedented Degree of Human Immunodeficiency Virus Type 1 (HIV-1) Group M Genetic Diversity in the Democratic Republic of Congo Suggests that the HIV-1 Pandemic Originated in Central Africa. Journal of Virology. 2000. 74: 10498-10507

Wyatt R and Sodroski J. The HIV-1 envelope glycoproteins: fusogens, antigens, and immunogens. Science. 1998. 280(5371): 1884-1888

Wei X, Ghosh SK, Taylor ME, Johnson VA, Emini EA, et al. Viral dynamics in human immunodeficiency virus type 1 infection. Nature. 1995. 373: 117-112

Weller I, Crawford DH, Iliescu V, MacLennan K, Sutherland S, Tedder RS, and Adler MW. Homosexual men in London: Lymphadenopathy, immune status, and Epstein-Barr virus infection. Edited by Selikoff IJ, Teirstein AS, and Hirschman SZ. Annals of the New York Academy of Science. 1984. 437: 248-249

Williamson C, Engelbrecht S, Lambrick M, Janse van Rensburg E, Wood R, Bredell W, and Williamson A. HIV-1 subtypes in different risk groups in South Africa. Lancet. 1995. 346: 782 
WHO / World Health Organization (1983), Acquired Immune Deficiency Syndrome Emergencies. Report of a WHO Meeting, Geneva. 22-25, November

Yang Z, Goldman N, and Friday A. Comparison of models for nucleotide substitution used in maximum likelihood phylogenetic estimation. Molecular Biology and Evolution. 1994. 11: 316-324

Zhang M, Schultz AK, Calef C, Kuiken C, Leitner T, Korber B, Morgenstern B, and Stanke M. jpHMM at GOBICS: a web server to detect genomic recombinations in HIV-1. Nucleic Acid Research. 2006. 34: 463-5

zur Megede J, Engelbrecht S, de Oliveira T, Cassol S, Scriba TJ, Janse van Rensburg E, and Barnett SW. Novel Evolutionary Analyses of Full-Length HIV Type 1 Subtype C Molecular Clones from Cape Town, South Africa. AIDS Research and Human Retroviruses. 2002. 18: 1327-1332 


\section{CHAPTER SIX - APPENDICES}

Table of Content

Page

APPENDIX A Sequenced data of the subgenomic regions

Partial gag sequences

Partial pol sequences

Partial env sequences

150

APPENDIX B REGA and jpHMM subtyping results of subgenomic regions

APPENDIX C List of sequencing primers used for the sequencing of near full-length genomes

APPENDIX D Gene Cutter Results of NFLG fragments

APPENDIX E REGA and jpHMM subtyping results of NFLG fragments 


\section{Appendix A}

\section{Partial gag sequences}

$>$ R84_gag

GCATGGGTAAAAGTAGTAGAAGAGAAGGCTTTCAGCCCAGAAGTGATACCCATGTTTTCAGCATTATCA GAAGGAGCCACCCCACAAGATTTAAACACCATGCTAAACACAGTGGGGGGACATCAAGCAGCCATGCAA ATGTTAAAAGAGACCATCAATGAGGAAGCTGCAGAATGGGATAGATTGCATCCAGTGCATGCAGGGCCT ATTGCACCAGGCCAGATGAGAGAACCAAGGGGAAGTGACATAGCAGGAACTACTAGTACCCTTCAGGAA CAAATAGGATGGATGACAAATAATCCACCTATCCCAGTAGGAGAAATCTATAAAAGATGGATAATCCTA GGATTAAATAAAATAGTAAGGATGTATAGCCCTGTCAGCATTCTGGACATAAGACAAGGACCAAAGGAA CCCTTTAGAGACTATGTAGACCGGTTCTATAAAACTCTAAGAGCCGAACAAGCTTCACACAAGGT

$>$ TV86_gag

TGGGTAAAAGTAATAGAGGAGAAGGCTTTCAGCCCAGAAGTAATACCCATGTTTACAGCATTATCAGAA GGAGCCACCCCACAAGATTTAAACACCATGTTAAATACAGTGGGGGGACATCAAGCAGCCATGCAAATG TTAAAAGATACCATCAATGAAGAGGCTGCAGAATGGGATAGGTTACATCCAGTGCAGGCAGGGCCTATT GCACCAGGCCAGATGAGAGAACCAAGGGGAAGTGACATAGCAGGAACTACTAGTAACCTTCAGGAACAA ATAGCATGGATGACAGGTAATCCACCTATTCCAGTAGGAGACATCTATAAAAGATGGATAATTATAGGG TTAAATAAAATAGTAAGAATGTATAGCCCTGTCAGCATTTTGGACATAAAACAAGGGCCAAAGGAACCC TTTAGAGACTATGTAGATCGGTTCTTTAAAACTTTAAGAGCCGAAC

$>$ TV101_gag

AGGACTTTAAATGCATGGGTAAAAGTAATAGAAGAAAAGGCTTTCAGCCCAGAAGTAATACCCATGTTC TCAGCATTATCAGAAGGAGCCACCCCACAAGATCTAAATACAATGCTGAACGTAGTGGGGGGACACCAG GCAGCTATGCAGATGTTAAAAGACACCATCAATGAGGAAGCTGCAGAGTGGGACAGGTTACATCCACAA CATGCAGGGCCTATTCCACCAGGCCAGATAAGGGAACCAAGGGGAAGTGAYATAGCAGGAACTACCAGT ACCCCTCAAGAACAATTGCAATGGATGACAGGCAACCCACCTATCCCAGTGGGAGACATCTATAAAAGA TGGATAATCCTGGGATTAAATAAAATAGTAAGAATGTATAGCCCTGTTAGCATTTTGGATATAAGACAA GGGCCAAAAGAACCCTTCAGAGACTATGTAGATAGGTTCTTTAAAACTCTTAGAGCTGAGCAAGCTACA $\mathrm{CA}$

$>$ TV218_gag

AGAACCTTGAATGCATGGGTAAAAGTAGTAGAGGAGAAGGCTTTTAGCCCAGAGATAATACCCATGTTT ACAGCATTATCAGAAGGAGCCACCCCACAAGRTTTAAACACCATGCTAAATACGGTGGGGGGACATCAA GCAGCCATGCAGATGTTAAAAGATACAATCAATGAAGAGGCTGCAGAATGGGATAGATTACATCCAGTG CATGCAGGGCCTATTGCACCAGGCCAAATGAGAGAACCAAGGGGAAGTGACATAGCAGGAACTACTAGT ACCCTTCAGGAACAAATAGCATGGATGACAAGTAACCCACCTATTCCAGTGGGAGACATCTATAAAAGA TGGATAATTCTGGGATTAAATAAAATAGTGAGAATGTATAGCCCGGTCAGCATTTTGGACATAAGACAA 
GGACCAAAGgAACCCTTTAGAGACTATGTAGATCGgTTCTTTAAAACTCTAAGAGCTGAACAAGCTACA CA

$>$ TV239_gag

TTGAATGCATGgGTAAAAgTAATAGAAGAAAAGgCTTTCAgCCCAGAAgTAATACCCATgTTCTCAGCA TTATCAGAAGGAGCCACCCCGCAAGATTTAAATATGATGCTAAACATAGTGGGGGGACACCAGGCAGCT ATGCAAAAGTTAAAAGATACCATCAATGAGGAAGCTATAGAATGGGACAGGACACATCCAGTACATGCA GGGCCTATCCCACCAGGCCAGATGAGAGAACCAAGTGGAAGTGATATAGCAGGAACTACTAGTACCCTT CAAGAACAGATAGGATGGATGACAAGTAACCCACCTATCCCAGTGGGAGACATCTATAAAAGGTGGATA ATTCTGgGATTAAATAAAATAGTAAGAATGTATAGCCCTGTCAGCATTTTGGACATAAGACAAGgGCCA AAAGAACCCTTCAGAGACTATGTAGATAGGTTCTTTAAAGCTCTCAGAGC

$>$ TV314_gag

TTGAATGCATGgGTAAAAGTAATAGAAGAAAAGGCTTTCAGCCCTGAAgTAATACCCATGTTCTCAGCA TTATCAGAAGGAGCCACCCCACAAGATTTAAATATGATGCTGAACATAGTGGgGGGACACCAGGCAGCT ATGCAAATAAGTAACAGTGATACCATCAATGAGGAAGCTGCAGAATGGGATAGGCTACATCCAGTACAT GCAGGGCCAGTTGCACCAGGCCAGATGAGAGAACCAAGTGGAAGTGATATAGCAGGAACTACTAGTACC CCTCAAGAACAAATAGCATGGATGACAGGCAACCCACCTATCCCAGTGGGAGACATCTATAAAAGATGG ATAATCCTAGgGTTAAATAAAATAGTAAGAATGTATAGCCCTGTTAGCATTTTGGATATAAAACAAGgG CCAAAAGAACCCTTCAGAGACTATGTAGATAGGTTCTTTAAAACTCTCAGAGCCGA

$>$ TV412_gag

CTTTGAATGCATGgGTAAAAgTAATAGAAGAAAAGGCTTTCAGCCCAGAAgTAATACCCATGTTCTCAG CATTATCAGAAGgAGCCACCCCACAAGATTTAAATATGATGCTGAACATAGTGGGgGGACACCAGGCAG CCATGCAAAAGTTACCAAGATACCATTAATGAGGAAGCTGCAGAATGGGACAGAGTACATCCAGTACAT GCAGGGCCTATTCCACCAGGCCAGATGAGAGAACCAAGAGGAAGTGACATAGCAGGAACTACTAGTACC ATTCAAGAACAAATAGGATGGATGACAAGCAACCCACCTGTCCCAGTGGGAGAAATCTATAAAAGATGG ATAATCCTGGGATTAAATAAAATAGTAAGAATGTATAGCCCTGTTAGCATTTTGGATATAAAACAAGgG CCAAAAGAACCCTTCAGAGATTATGTAGATAGgTTCTTTAAAACTCTCAGAGCCGAACAAGCTTCACAA AAGGTTCTA

$>$ TV441_gag

GCATGGgTAAAAGTAGTAGAGGAGAAGgCTTTTAGCCCAGAGGTAATACCCATGTTTACAGCATTATCA GAAGGAGCCACCCCACAAGATTTAAACTCCATGCTAAATGCGGTGGGGGGGCATCAAGCAGCCATGCAA ATGTTAAAAGATACCATCAATGAAGAGGCTGCAGAATGGGATAGATTACATCCAGTACATGCAGGGCCC ATTGCACCAGGCCAAATGAGAGAACCAAGGGGAAGTGACATAGCAGGAACTACTAGTACCCTTCAGGAA CAAGTAGCATGGATGACAAGTAACCCACCTATTCCAGTGGGAGACATCTATAAAAGATGGATAATTCTG GGGTTAAATAAAATAGTAAGAATGTATAGCCCTGTCAGTATTTTGGACATAAGACAAGGGCCAAAGGAA CCCTTTAGAGACTATGTAGATCGGTTCTTTAAAACTTTAAGAGC 
>TV480_gag

GCATGGGTAAAAGTAGTAGAGGAGAAGGCCTTTAGCCCAGAGGTAATACCCATGTTTACAGCATTATCA GAAGGAGCCACCCCCTCTGATTTAAACTCCATGTTAAATGCGGTGGGGGGACATCAAGCAGCCATGCAA AAGTTAAAAGATACCATCAATGAAGAGGCTGCAGAATGGGATAGATTACATCCAGTACATGCGGGGCCT GTTGCACCAGGCCAAATGAGAGAACCAAGGGGAAGTGACATAGCAGGAACTACTAGTACCCTTCAGGAA CAAATAGCATGGAGTACAGCTAACCCAGCTATTCCAGTGGGAGAAATCTATAAAAGATGGATAATTCTG GGGCTAAATAAAATAGTGAGAATGTATAGCCCTGTCAGCATTTTGGACATTAGACAAGGGCCAAAGGAA CCCTTTAGAGACTATGTAGATCGGTTCTTTAAAACTTTAAGAGCCGAACAAGCTTCACA

$>$ TV515_gag

TTTGAATGCATGGGTAAAGGTGATAGAAGAGAAGGCTTTTAGCCCAGAAGTGATACCCATGTTCTCAGC ATTATCAGAAGGGGCCACCCCACAAGATTTAAACACCATGCTAAATACAGTAGGAGGACATCAAGCAGC CATGCAAATTTTAAAAGACACCATCAATGAGGAAGCTGCAGAATAGGACAGAGTACATCCACCACAGGC AAGGCCTCACCCACCAGGCCAGATAAAGGAACCTAGAGGAAGTGACATAGCTGGAACTACTAGTACCCT TCATGAACAGATACAATAGATGACAAGCAACCCACCTATCCCAGTAAGAGACATCTATAAAAGATAGAT CATCCTAAGATTAAATAAAATAGTAAGAATGTATAGCCCTGTCAGCATTCTGGACATAAAACAAAGGCC AAAGGAACCCTTTAGAGACTATGTAGATAGGTTCTTCAAAACTCTAAGAGCCGAACAA

\section{Partial pol sequences}

$>$ R84_pol

AGTAGATAAATTAGTCAGTGCTGGAATCAGGAGAGTACTATTTTTAGATGGGATAGATAAGGCCCAAGA AGAACATGAGAAATATCACAGTAATTGGAGAGCAATGGCTAGTGATTTTAACCTGCCACCTATAGTAGC AAAAGAGATAGTAGCCAGCTGTGATAAATGTCAGTTAAAAGGAGAAGCCATACATGGACAAGTAGACTG TAGTCCAGGAATATGGCAACTAGATTGTACACATTTAGAAGGAAAAGTTATCCTGGTAGCAGTTCATGT AGCCAGTGGATATATAGAAGCAGAAGTTATTCCAGCAGAGACAGGGCAGGAAACAGCATACTTTCTCTT AAAATTAGCAGGAAGATGGCCAGTAAAAACAATACATACAGACAATGGCAGCAATTTCACCAGTACTAC GGTTAAGGCCCCCTGTTGGTGGGCGGGGATCAAGCAGGAATTTGGCATTCCCTACAATCCCCAAAGTCA AGGAGTAGTAGAATCTATGAATAAAGAATTAAAGAAAATTATAGGACAGGTAAGAGATCAGGCTGAACA TCTTAAGACAGCAGTACAAATGGCAGTATTCATCCACAATTTTAAAAGAAAAGGGGGGATTGGGGGGTA CAGTGCAGGGGAAAGAATAGTAGACATAATAGCAACAGACATACAAACTAAAGAATTACAAAAACAAAT TACAAAAATTCAAAATTTTCGGGTTTATTACAGGGACAGCAGAGAGCCACTTTGGAAAGGACCAGCAAA GCTTCTCTGGAAAGGTGAAGGGGCAGTAGTAATACAAGATAATAGTGACATAAAAGTAGTGCCAAGAAG AAAAGTAAAAATCATTAGGGATTATGGAAAACAGATGGCAGGTGATGATTGTGTGGCAAGTAGACAGGA TGAGGATTAGAACATGGAACAGCTTAGTAAAACACCATATGTAT

>TV86_pol

GTCAGTAAAGGAATCAGGAAAGTGCTGTTTCTAGATGGAATAGATAAGGCTCAAGAAGAGCATGAAAAG TATCACAGCAATTGGAGAGCAATGGCTAGTGAGTTTAATATGCCACCCGTAGTAGCAAAAGAAATAGTA GCTAGCTGTGATAAATGTCAGCTAAAAGGGGAGGCCATGCATGGACAAGTAGACTGTAGTCCAGGAATA 
TGGCAATTAGATTGTACACATTTAGAAGGAAAAGTCATCCTGGTAGCAGTCCATGTAGCTAGTGGCTAC ATAGAAGCAGAGGTTATCCCAGCAGAAACAGGACAAGAAACAGCATACTATATACTAAAATTAGCAGGA AGATGGCCAGTCAAAGTAATACATACAGACAATGGCAGTAATTTTACCAGTACTCCAGTTAAGGCAACC TGTTGGTGGGCAGGTATCCAACAGGAATTTGGAATTCCCTACAATCCCCAAAGTCAGGGAGTAGTAGAA TCCATGAATAAAGAATTAAAGAAAATAATAGGACAAGTAAGAGATCAAGCTGAGCACCTTAAGACAGCA GTACAAATGGCAGTATTCATTCACAATTTTAAAAGAAAAGGGGGGATTGGGGGGTACAGTGCAGGGGAA AGAATAATAGACATAATAGCAACAGACATACAAACTAAAGAATTACAAAAACAAATTACAAAAATTCAA AATTTTCGGGTTTATTACAGAGACAGCAGAGACCCTATTTGGAAAGGACCAGCCAAACTACTCTGGAAA GGTGAAGGGGCAGTAGTAATACAAGATAACAGTGACATAAAGGTAGTACCAAGGAGGAAAGCAAAAATC ATTAGGGATTATGGAAAACAGATGGCAGGTGATGATTGTGTGGCAGGTAGACAGGATGAAGATTAGAAC ATGGAATAGCTTAGTAAAACACCATA

$>$ TV101_pol

AACAARTAGATAAATTAGTTAGCTCTGGGATCAGGAAGGTACTATTTTTAGATGGGATAGACAAGGCTC AAGAAGACCATGAGAGRTATCACAGCAATTGGAGAACAATGGCTAGTGATTTTAATCTRCCACCTATAG TAGCAAAGGAAATAGTAGCCAGCTGTGATAAATGTCAGCTAAAAGGAGAAGCCATGCATGGACAAGTAG ACTGTAGTCCAGGAATATGGCAATTAGACTGTACACATTTAGAGGGAAAGTTATCCTGGTAGCAGTCC ATGTAGCCAGTGGCTATATAGAAGCAGAAGTTATTCCAGCAGAAACAGGACAGGAAACAGCCTATTTTA TCTTAAAATTAGCAGGAAGATGGCCAGTAAAAGTAGTACATACAGACAATGGCAGCAATTTCACTAGCA CTGCAGTTAAAGCAGCCTGTTGGTGGGCAAATGTCCAACAGGAATTTGGGATTCCCTACAATCCCCAAA GTCAGGGAGTAGTAGAATCTATGAATAAAGAATTAAAGAAAATYATAGGGCAGGTAAGAGATCAAGCTG AACATCTTAAAACAGCAGTACAAATGGCAGTGTTCATTCACAATTTTAAAAGAAAAGGGGGGATTGGGG GATACAGTGCAGGGGAAAGAATAATAGACATAATAGCAACAGACATACAAACTAAAGAATTACAAAAAC AYATTTCAAAAATTCAAAATTTTCGGGTTTATTACAGGGACAGCAGAGATCCAATTTGGAAAGGACCAG CAAARCTKCTCTGGAAAGGTGAAGGGGCAGTGGTAATACAAGACARTAGTGAAATAAAGGTAGTGCCAA GAAGGAAAGCAAAGATCATTAGGGATTATGGAAAACAGATGGCAGGTGATGATTGTGTGGCAGGTAGAC AGGATGAGGATTAGAACATGGCACAGTTTAATAAAACACCATATGTAT

>TV218_pol

AACAAGTAGATAAATTAGTAAGTAAAGGGATCAGRAAAGTGCTGTTTCTAGATGGAATAGATAAGGCTC AAGAAGATCATGAAAGATATCACAGCAATTGGAGRGCAATGGCTAGTGAGTTTAATCTGCCACCCATAG TAGCAAAAGAAATAGTAGCTAGCTGTGATAAATGTCAGYTAAAAGGGGAAGCCATACATGGACAGGTAG ACTGTAGCCCGGGGATATGGCAATTAGACTGTACACATTTAGAAGGAAAAATYATCCTGGTAGCAGTCC ATGTAGCCAGTGGCTACATAGAAGCAGAGGTTATCCCAGCAGAAACAGGACAAGAAACAGCATACTTTA TACTAAAATTAGCAGGAAGATGGCCAGTCAAAGTAATACATACAGACAATGGCAGTAATTTCACCAGTG CTGCAGTTAAGGCAGCCTGTTGGTGGGCAGGTATCCAACAGGAATTTGGGATTCCCTACAATCCCCAAA GTCAGGGAGTGGTAGAATCCATGAATAAAGAATTAAAGAAAATCATAGGGCAGGTAAGAGAYCAAGCTG AGCACCTTAAGACAGCAGTGCAAATGGCAGTATTCATTCACAATTTTAAAAGAAAAGGGGGGATTGGGG GGTACAGTGCAGGGGAAAGAATAATAGACATAATAGCAACAGACATACAAACTAAAGAATTACAAAAAC AAATTACAAAAATTCAAAATTTTCGGGTTTATTACAGAGACAGCAGAGACCCTATTTGGAAAGGACCAG 
CCAAACTACTCTGGAAAGGTGAAGGAGCAGTAGTAATACAAGATAACAGTGACATCAAGGTAGTACCAA GGAGGAAAGCAAAAATCATTAA GGACTATGGAAAACAGATGGCAGGTGCTGATTGTGTGGCAGGTAGACAGGATGAAGATTAGAACATGGA ATAGTTTGGTAAAGCACCATATGCAT

>TV239_pol

AAGTAGATAAATTAGTCAGTTCTGGAATCAGGAAGGTGCTATTTTTAGATGGGATAGATAAGGCTCAAG AAGAGCATGAAAGGTATCACAGCAATTGGAGAGCAATGGCTAGTGATTTCAACCTGCCACCAGTAGTAG CAAAGGAAATAGTAGCCAGCTGTGATAAATGTCAACTAAAAGGGGAAGCCATGCATGGACAAGTAGACT GTAGTCCAGGAATATGGCAAGTAGATTGCACACATCTAGAAGGAAAAGTAATCATAGTAGCAGTCCATG TAGCCAGTGGCTATATAGAGGCAGAAGTTATCCCAGCAGAAACAGGACAGGAGGCAGCATACTTTCTGT TAAAATTAGCAGCAAGATGGCCAGTAAAGGTAATACACACAGACAATGGCAGTAATTTCACCAGTGCCG CAGTTAAAGCAGCCTGTTGGTGGGCAAATATCCAACAGGAATTTGGAATTCCCTACAATCCCCAAAGTC AAGGAGTAGTGGAATCTATGAATAAAGAATTAAAGAAAATCATAGGGCAGGTAAGAGAGCAAGCTGAGC ACCTTAAGACAGCAGTACAAATGGCAGTATTCATTCACAATTTTAAAAGAAAAGGGGGGATTGGGGGGT ACAGTGCAGGGGAAAGAATAATAGACATAATAGCAACAGACATACRAACTAAAGAATTACAAAAACAAA TTACAAAAATTCAAAATTTCCGGGTTTATTACAGGGACAGCAGAGACCCAATTTGGAAAGGACCAGCAA GACTACTCTGGAAAGGTGAAGGGGCAGTAGTAATACAGGACAATAGTGATATAAAGGTAGTACCAAGAA GAAAAGCAAAAATCATTAGGGACTATGGAAAACAGATGGCAGGTGATGATTGTGTGGCAGGTAGACAGG ATGAGGATTAGAACATGGCACAGCTTAGTAAAACACCATATGTAT

$>$ TV314_pol

AGTAGATAAATTAGTCAGTTCTGGAATCAGGAAGGTGCTATTTCTAGATGGGATAGATAAGGCTCAAGA AGAACATGAAAGATATCACAGCAACTGGAGAGCTATGGCTAGTGATTTTAATCTGCCACCTATAGTAGC AAAGGAGATAGTAGCCAGCTGTGATAAATGCCAGCTAAAAGGGGAAGCCATGCATGGACAAGTAGACTG CAGTCCAGGAATATGGCAATTAGACTGCACACATCTAGAAGGAAAAGTAATTCTGGTAGCAGTCCATGT AGCCAGTGGCTATATAGAAGCAGAAGTTATCCCAGCAGAAACAGGACAAGAGACAGCATACTTTCTATT AAAATTAGCAGGAAGATGGCCAGTAAAAACAGTACACACAGACAATGGCAGCAATTTCACCAGTGCTGC AGTTAAAGCAGCCTGTTGGTGGGCAGGTATCAAACAGGAATTTGGAATTCCCTACAATCCCCAAAGTCA AGGAGTAGTGGAATCTATGAATAAGGAATTAAAGAAAATCATAGGGCAGGTAAGAGAGCAAGCTGAACA CCTTAAGACAGCAGTACAAATGGCAGTATTCATTCACAATTTTAAAAGAAAAGGGGGGATTGGGGGGTA CAGTGCAGGGGAAAGAATAATAGACATAATAGCAACAGACATACAAACTAAAGAATTACAAAAACAAAT TACAAAAATTCAAAAATTTCGGGTTTATTACAGGGACAGCAGAGATCCAATTTGGAAAGGACCAGCAAA ACTACTCTGGAAAGGTGAAGGGGCAGTGGTAATACAGGACAATAGTGATATCAAAGTAGTACCAAGAAG AAAAGCAAAGATCCTTAGGGAT

TATGGACAACAGATGGCAGGTGATGATTGTGTGGCAGGTAGACAGGATGAGGATTAGAACATGGCACAG CTTAGTAAAACACCATATGTAT 
>TV340_pol

AGTAGATAAATTAGTCAGTAGTGGAATCAGAAAAGTACTATTTCTAGATGGCATAGATAAAGCCCAAGA AGAGCATGAAAGATATCACAGCAATTGGAGGGCAATGGCTAATGACTTTAATCTGCCACCTATAGTAGC AAAAGAAATAGTGGCCAGCTGTGATAAGTGCCAGCTAAAAGGGGAAGCCATGCATGGACAAGTAGACTG TAGTCCAGGAATATGGCAATTAGATTGTACACATTTAGAAGGAAAAATTATCCTGGTAGCAGTCCATGT AGCCAGTGGCTATATAGAAGCAGAAGTTATCCCAGCAGAAACAGGACAGGAAACAGCATACTTTATATT AAAATTAGCAGGAAGATGGCCAGTAAAAGTAATACACACAGATAATGGCAGCAATTTCACCAGCAGTGC AGTAAAGGCAGCATGTTGGTGGGCAAATATCACACAAGAATTTGGAATTCCCTACAATCCCCAAAGCCA AGGAGTAGTAGAGTCTATGAATAAAGAATTAAAGAAAATTATAGGACAGGTCAGAGATCAAGCTGAACA CCTTAAGACAGCAGTACAGATGGCAGTATTCATTCATAATTTTAAAAGAAAAGGGGGGATTGGGGGGTA CAGTGCAGGGGAAAGAATAGTAGACATAATAGCATCAGATATACAAACTAAAGAACTACAAAAACAAAT TATAAAAATTCAAAATTTTCGGGTTTATTACAGGGACAGCAGAGACCCAATTTGGAAAGGACCAGCAAA ACTACTCTGGAAAGGTGAAGGGGCAGTAGTAATACAGGACAATAGTGATATAAAAGTAGTACCAAGAAG AAAAGCAAAGATCATTAGGGATTATGGAAAACAGATGGCAGGTGATGATTGTGTGGCAGGTAGACAGGA TGAGGATTAATACATGGAAAAGCTTAGTAAAACACCATATG

$>$ TV412_pol

AGTAGATAAATTAGTCAGTAATGGAATCAGGAAGATACTATTTTTAGATGGGATAGATAAGGCTCAAGA AGAACATGAAAGATATCATAGCAATTGGAGAGCAATGGCTAATGATTTTAACCTGCCACCTGTGGTAGC AAAGGAAATAGTAGCCAGCTGTGATAAATGTCAGCTAAAAGGGGAAGCCATGCATGGACAGGTAGACTG TAGTCCAGGAATATGGCAATTAGATTGCACACATCTAGAAGGAAAAGTAATTCTGGTAGCAGTTCATGT AGCCAGTGGCTATATAGAAGCAGAAGTTATCCCAGCAGAAACAGGACAGGAAACAGCATACTTTCTGCT AAAATTAGCAGGGAGGTGGCCAGTAAAAGTAGTTCACACAGACAATGGCAGCAATTTCACCAGTGCTGC AGTTAAAGCAGCCTGTTGGTGGGCAAATATCCAACAGGAATTTGGGATTCCCTACAATCCCCAAAGTCA AGGAGTAGTAGAATCTATGAATAAAGAATTAAAGAAAATTATAGGACAGGTAAGAGATCAAGCTGAACA TCTTAAGACAGCAGTACAAATGGCAGTATTCATTCACAATTTTAAAAGAAAAGGGGGGATTGGGGGGTA CAGTGCAGGGGAAAGAATAATAGACATAATAGCAACAGACATACAAACTAAAGAACTACAAAAACAAAT TACAAAAATTCAAAATTTTCGGGTTTATTACAGGGACAGCAGAGATCCAGTTTGGAAAGGACCAGCAAA GCTTCTCTGGAAAGGTGAAGGGGCAGTAGTAATACAAGACAATAGTGAAATAAAGGTAGTACCAAGAAG AAAAGCAAAGATCATTAGGGATTATGGAAAACAGATGGCAGGTGATGATTGTGTGGCAGGTAGACAGGA TGAGGATTAGAACATGGAACAGCTTAGTAAAACACCATATGTAT

>TV441_pol

CAAGTAGATAAATTAGTCAGTAGTGGAATCAGGAAAGTATTGTTCCTAGATGGAATAGATAAGGCTCAA GAAGAGCATGAAAAATATCACAATAATTGGAGAGCAATGGCTGCTGATTTTAATCTGCCACCTGTAGTA GCAAAAGAAATAGTAGCTAGCTGTGATAAGTGTCAGCTAAAAGGGGAAGCCATGCATGGACAAGTAGAC TGTAGTCCAGGAATATGGCAATTAGATTGCACTCATCTAGAAGGGAAGTTATCCTGGTAGCAGTCCAT GTAGCCAGTGGCTATATGGAAGCAGAAGTTATCCCAGCAGAAACAGGACAGGAGACAGCATACTTTATA TTAAAGCTAGCAGGAAGGTGGCCAGTAAGAGTAATACATACAGACAACGGCCCCAATTTCACCAGTGCA GCAGTTAAGGCAGCCTGTTGGTGGGCAAATATCCAACAGGAATTTGGGATTCCCTACAACCCCCAAAGT CAAGGAGTAGTGGAATCTATGAATAAAGAATTAAAGAAAATCATAGGGCAGGTAAGAGATCAAGCTGAG 
CACYTTAAGACAGCAGTACAAATGGCAGTATTCATTCACAATTTTAAAAGAAAAGGGGGGATTGGGGGG TACAGTGCAGGGGAAAGAATAATAKACATAATAGCAACAGACATACAAACTAAAGAATTACAAAAACAA ATTATAAAAATTCAAAATTTTCGGGTTTATTACAGAGACAGCAGAGACCCTATTTGGAAAGGACCAGCC AAACTACTCTGGAAAGGTGAAGGGGCAGTAGTAATACAAGACAACAGTGACATAAAGGTAGTACCAAGG AGGAAAGTAAAAATCATTAAGGACTATGGAAAACAGATGGCAGGTGCTGATTGTGTGGCAGGTAGACAG GATGAGATTAGAACATGGAATAGCTTAGTAAAACACCATATGTAA

$>$ TV480_pol

AACAAGTAGATAAATTAGTCAGTAATGGAATCAGGAAGGTGCTGTTTCTAGATGGAATAGATAAGGCTC AAGAAGAGCATGAAAAATATCACAGCAATTGGAGAGCAATGGCTAGTGAGTTTAACCTGCCACCCATAG TAGCAAAAGAAATAGTAGCTAGCTGTGATAAATGTCAGCTAAAAGGGGAAGCCATACATGGACAAGTAG ACTGTAGTCCAGGGATATGGCAATTAGATTGTACACATTTAGAAGGAAAAGTCATCCTGGTAGCAGTCC ATGTAGCCAGTGGCTACATAGAGGCAGAGGTTATCCCAGCAGAAACAGGACAAGAAACAGCATACTATA TACTGAAATTAGCAGGAAGATGGCCAGTCAAAGTGATACATACAGACAATGGCAGTAATTTCACCAGTG CTGCAGTTAAGGCAGCCTGTTGGTGGGCAGGTATCCAACAGGAATTCGGGATTCCCTACAATCCCCAAA GTCAGGGAGTAGTAGAATCCATGAATAAAGAATTAAAGAAAATCATAGGGCAGGTAAGAGAGCAAGCAG AGCACCTTAAGACAGCAGTACAAATGGCAGTGTTCATTCACAATTTTAAAAGAAGAGGGGGGATTGGGG GGTACAGTGCAGGGGAAGAATAATAGACATAATAGCAACAGACATACAAACTAAAGAATTACAAAAAC AAATTATAAAAATTCAAAATTTCCGGGTTTATTACAGAGACAGCAGAGACCCTATTTGGAAAGGACCAG CCAAACTACTCTGGAAAGGTGAAGGGGCAGTAGTAATACAAGATAACAGTGACATAAAGGTAGTACCAA GGAGGAAAGTAAAAATCATTAAGGACTATGGAAAACAGATGGCAGGTGCTGATTGTGTGGCAGGTAGAC AGGATGAGGATTAGAACTTGGAATAGCTTAGTAAAACACCATATGTAT

>TV515_pol

AGTAGATAAATTAGTCAGTGCTGGAATCAGAAAAGTACTGTTTTTAGATGGGATAGATAAGGCACAAGC AGAACATGAAAAATATCACAACAATTGGAGAGCAATGGCTAGTGACTTTAATCTGCCCCCTGTAATAGC AAAAGAAATAGTAGCTAGCTGTGATAAATGTCAGCTAAAAGGGGAGCTATGCATGGACAAGTAGACTG TAGCCCAGGGATATGGCAATTAGATTGCACACATTTAGAAGGAAAAATTATCCTAGTAGCTGTTCATGT AGCTAGTGGATATATAGAAGCAGAAGTCATTCCAGCAGAAACAGGACAGGAAACTGCCTACTACATACT AAAGTTAGCAGGAAGATGGCCAGTAAAAATAATACATACAGACAATGGCAGCAATTTCACCAGTACTGC GGTTAAGGCAGCCTGTTGGTGGGCAGGTATCCAGCAAGAATTTGGAATTCCCTACAATCCCCAAAGTCA AGGAGTAGTAGAATCTATGAATAAAGAATTAAAGAAAATCATAGGACAAGTAAGAGATCAAGCTGAACA TCTTAAAACAGCAGTACAAATGGCAGTATTCATTCATAATTTTAAAAGAAAAGGGGGGATTGGGGGGTA CAGTGCAGGGGAAAGAATAATAGACATAATATCAACAGACATACAAACTAAAGAACTACAAAAACAAAT TATAAAAATTCAAAATTTCCGGGTTTATTACAGGGACAGCAGAGACCCAGTTTGGAAAGGACCAGCAAA GCTACTGTGGAAAGGTGAAGGGGCAGTAGTCATACAAGACAATAGTGAAATAAAAGTAGTGCCAAGAAG GAAAGTAAAGATCATTAGGGATTATGGAAAACAGATGGCAGGTGATGATTGTGTGGCAAGTAGACAGGA TGAGGATTAACACATGGAAAAGCTTAGTAAAACACCATATG 


\section{Partial env sequences}

>R84_env

CAAAACAATTTGCTGAAGGCTATTGAGGCGCAACAGCACCTGTTGCAACTCACAGTCTGGGGCATCAAG CAGCTCCAGGCAAGAGTCCTGGCTATAGAAAGATACCTAAAGGATCAACAGCTCCTGGGGATTTGGGGT TGCTCTGGAAAACTCATTTGCACCACTGCTGTGCCTTGGAATGCTAGTTGGAGTAATAAATCTCAGGAT AAGATTTGGCATAACATGACCTGGATGGAGTGGGAAAGAGAAATTAGCAATTACACAAGCTTAATATAC ACCTTAATTGAAGAATCGCAGAACCAACAAGAAAAGAATGAACAAGAATTGTTGGAATTGGATCAATGG GCAAATTTGTGGAATTGGTTTGACATAACAAAATGGCTGTGGTATATAAAAATATTYATAATGATA

>TV_86_env

CAAAGCAATTTGCTGAAGGCTATAGAGGCGCAACAGCATATGTTGCAACTCACGGTCTGGGGCATTAAG CAGCTCCAGGCAAGAGTCCTGGCTATAGAAAGATACCTAAAGGATCAACAGCTCCTAGGGATTTGGGGC TGCTCTGGAAAACTCATCTGCACCACTAATGTGCCTTGGAACACAAGTTGGAGTAGTAAATCTGAAGAT GAGATTTGGAATAACATGACTTGGATGCAGTGGGATAGAGAAATTAGTAATTACACAGGCATAATATAC CATTTGCTTGAAGACTCGCAAAACCAGCAGGAAAGGAATGAAAAAGACTTGTTTGCATTGGACAGTTGG AAAAATCTGTGGAATTGGTTTGACATATCAAAATGGCTGTGGTATATAAAAATATTYATAATGATA

>TV_101_env

CTGGCATAGTGCAACAGCAAAGCAATTTGCTGAGGGCTATAGAGGCTCAACAGCATCTGTTGAAGCTCA CGGTCTGGGGCATTAAACAGCTCCAGGCAAGAGTCCTGGCTSTGGAAAGATACCTAAAGGATCAACAGC TCCTAGGAATTTGGGGCTGCTCTGGAAAACTCATCTGCACCACTACTGTGCCCTGGAACTCTAGTTGGA GTAAYAAATCCCAGAATGAAATATGGGACAACATGACCTGGATGCAATGGGATAAAGAAATTAGCAATT ACACACAGATAATATATAGTCTAATTGAAGAATCACAAAACCAGCAGGAAAAGAATGAACAAGAGTTAC TGGCATTGGACAAGTGGGCAAATCTGTGGAATTGGTTTGATATATCAAATTGGCTGTGGTACATAAAGA TATTTATAATGATAGTAGGAGGCTTAATAGGATT

>TV_218_env

CTGGCATAGTGCGGCAGCAAAGCAATTTGCTGCAGGCTATAGAGGCTCAACAACATCTGTTGARACTCA CAGTCTGGGGCATTAAACAGCTCCAGGCAAGAGTCCTGGCTCTGGAAAGATACCTACAGGATCAACAGC TCCTAGGAATTTGGGGCTGCTCTGGAAAACTTATCTGCACCACTACTGTGCCTTGGAACTCTAGTTGGA GTAACAAATCTTATRAKGACATTTGGGRAAACATGACCTGGTTGCAGTGGGATAGAGAAATTAGCAATT ACACAAACACAATATACAGGCTACTTGAGGAGTCACAGAACCAGCAGGAAATTAATGAACAAGATTTAT TGGCCTTGGACAAGTGGGCAGGTCTGTGGAGTTGGTTTAGYATATCAAATTGGCTGTGGTATATAAAAA TRTTTATAATGATAGTAGGAGGCTT

>TV_239_env

TTGCTGAGGGCTATAGAGGCTCAACAGCATCTGTTGAAACTCACAGTCTGGGGCATTAAACAGCTCCAG GCAAGAATCCTGGCTGTGGAAAGATACCTAAAGGATCAACAGCTTCTAGGAATTTGGGGCTGCTCAGGA AAGTTAATCTGCACCACTGCTGTGCCCTGGAACTCTAGTTGGAGTAATAAATCTTATAATGAAATATGG GATAACATGACCTGGATGCAATGGGAAAAGGAAATTGACAATTACACAGGCATAATATATACTCTAATT 
GAAGAATCGCAGAACCAACAGGAAAAGAATGAACAAGATTTATTGGCATTGGACAAGTGGGCAAGTCTG TGGAATTGGTTTGACATATCAAATTGGCTATGGTATATAAAAAT

>TV_314_env

CAAAGCAATTTGCTGAGGGCTATAGAGGCTCAACAGCATCTGTTGAAACTCACAGTCTGGGGCATTAAA CAGCTCCAGGCAAGAGTCCTGGCTCTAGAGAGATACCTAAGGGATCAACAGCTCCTAGGAATTTGGGGC TGCTCTGGAAAACTCATTTGCGCCACTAATGTGCCTTGGAACTCTAGTTGGAGTAATAAATCTTATAAT GAAATATGGGATAACATGACCTGGCTGCAGTGGGATAAAGAAATTGACAATTACACAGAAACAATATAT AGGCTAATTGAAGAATCGCAAAACCAGCAGGAAAAGAATGAACAAGACTTATTGGCATTGGACAAGTGG ACAAATCTGTGGAGTTGGTTTGACATATCGAACTGGCTGTGGTATATAAAAATATTYATAATGATA

>TV_340_env

CAGAGCAATCTGCTGAGGGCTATAGAGGCTCAACAGCATTTGTTGAAACTCACAGTCTGGGGCATTAAA CAGCTCCAGGCAAGAGTCCTAGCTATAGAAAGATACCTAAGGGATCAACAGCTCCTAGGAATCTGGGGA TGCTCTGGAAAACTCATCTGCCCCACTAATGTGCCCTGGAACTCCAGCTGGAGTAATAAGTCTCAGAGT GAAATATGGGATAACATGACCTGGGTGCAATGGGATAAAGAAATTAGCAATTACACACAATTAATATAT GGTCTACTTGAGGAATCGCAGAACCAGCAGGAAAAGAATGAACAGGACTTATTGGCATTGGACAAGTGG GCAAGCCTGTGGAATTGGTTTGATATATCAAATTGGCTGTGGTACATAAAAATATTYATAATGATA

>TV_412_env

CAAAGCAATTTGCTGAGGGCTATAGAGGCTCAACAACATCTGTTGAAACTCACGGTCTGGGGCATTAAA CAGCTCCGGGCAAGAGTCCTGGCTGTGGAAAGATACCTAAAGGATCAACAGCTCCTAGGAATTTGGGGC TGCTCTGGAAAACTCATCTGCACCACTAATGTGCCCTGGAATTCTAGTTGGAGTAATAAATCTCAGGAG GAGATATGGGGGAACATGACCTGGCTGCAATGGGATAAAGAAGTTAACAATTATACAGAATTAATATAC TCCCTAATTGAAGAATCGCAGATCCAGCAGGAAAAGAATGAACAAGACTTATTGGCATTGGACAAATGG GCAAATCTGTGGAGTTGGTTTAGCATATCAAATTGGCTGTGGTATATAAGAATATTTATAATGATA

>TV_441_env

CAAAGCAATTTGCTGCAGGCTATAGAGGCTCAACAACATCTGTTGAAACTCACTGTCTGGGGCATTAAA CAGCTCCAGGCAAGAGTCCTGGCTCTGGAAAGATACCTAAAGGATCAACAGCTCCTAGGAATTTGGGGC TGCTCTGGAAAACTTATCTGCACCACTACTGTGCCTTGGAACTCTAGTTGGAGTAATAAATCTTATAAT GAGATTTGGGATAACATGACTTGGTTGCAGTGGGATAGAGAAATTAGCAATTACACAGAAACAATATAC AGGCTACTCCAAGACTCACAAATCCAGCAGGAACAGAATGAAAARGAGTTATTGGAATTGGACAAGTGG GCAAATCTGTGGAATTGGTTTGACATATCAAAGTGGCTATGGTACATAAAAATATTYATAATGATA

>TV_515_env

CAGAACAATCTGCTGAGGGCTATTGAAGCGCAACAGCATCTGTTGCAGCTCACAGTCTGGGGCATTAAA CAGCTCCAGGCAAGAGTCCTGGCTGTGGAAAGATACCTAAAGGATCAACAGCTCCTAGGGATTTGGGGC TGCTCTGGAAAACTCATCTGCACCACTAATGTGCCGTGGAACTCTAGTTGGAGTAATAGATCTCTGGAA GACATTTGGGAAAACATGACCTGGAGGGAGTGGGAAAAAGAGATTGGTAATTACTCAAACATAATATAT 
AGgTTAATTGAACAATCGCAGAACCAGCAGGAAATAAATGAAAAAGACTTATTGGCATTGGACAAGTGG GCAAGTCTGTGGAATTGGTTTGACATAACAAGCTGGCTGTGGTATATAAAAATATTYATAATGATA 


\section{Appendix B}

\begin{tabular}{|c|c|c|c|c|c|}
\hline Name & Length & Report & Assiggunent & Support & Gellome \\
\hline R84_gag & 4796p & Report & EllV-1 Subtype B & 90.0 & (n) \\
\hline Tr86 gag & 4600p & Report & EllV-1 Subtype C & 100.0 & "1" \\
\hline TV101_gag & 485bp & Repost & EIV-1 Subtype \& (A1) & 99.0 & (I" \\
\hline TV218_gag & 4856p & Report & IIV-1 Subtype C & 100.0 & (In) \\
\hline TV239_gag & 4646p & Report & ElV-1 Subtype A(A1) & 98.0 & 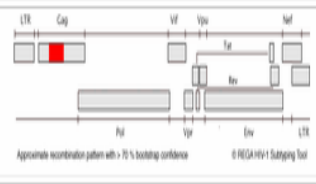 \\
\hline TV314__ag & 470bp & Report & HIV-1 Subtype A (Al) & 100.0 & ב" \\
\hline TV412 gag & $4926 p$ & Report & EIV-1 Subtype \& (AII) & 99.0 & 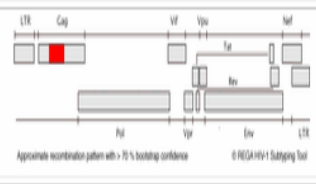 \\
\hline TV441_gag & 4580p & Report & HVIV-1 Subtype C & 100.0 & "1" \\
\hline TV480_gag & 4736p & Report & EllV-1 Subtype C & 100.0 & "1" \\
\hline TV515_gag & $4726 \mathrm{pp}$ & Report & $\mathbb{Z I V}$-1 Subtype F(F1) & 98.0 & 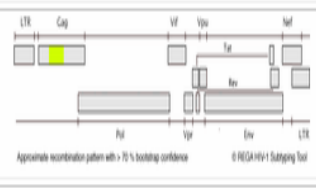 \\
\hline
\end{tabular}

Figure 6.1: REGA subtyping results of the gag p24 sequences. All data are included in the report including the size of each fragment, the subtype and the bootstrap values. 


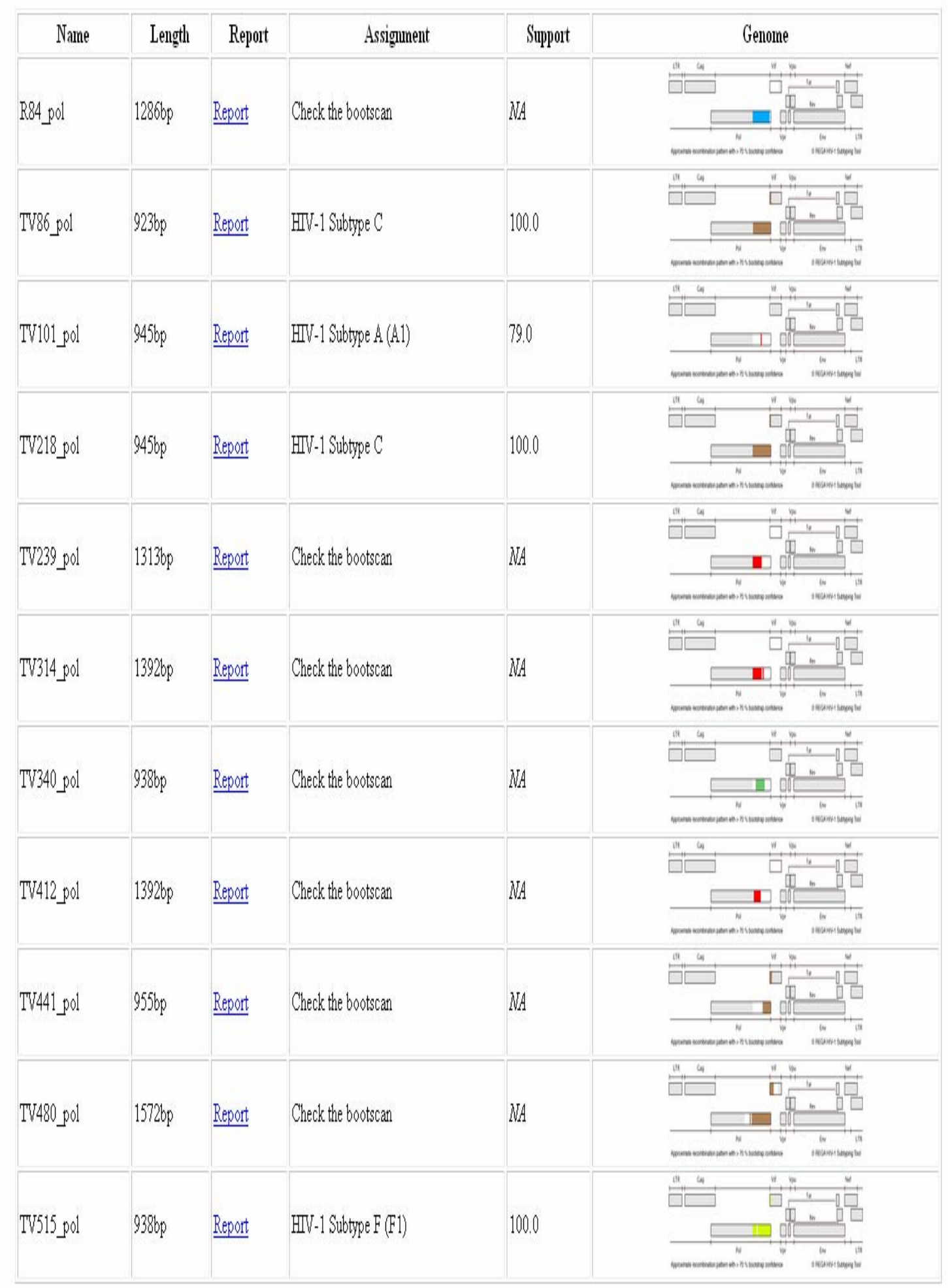

Figure 6.2: REGA subtyping results of the pol - integrase sequences. The subtype and the bootstrap values are included. 


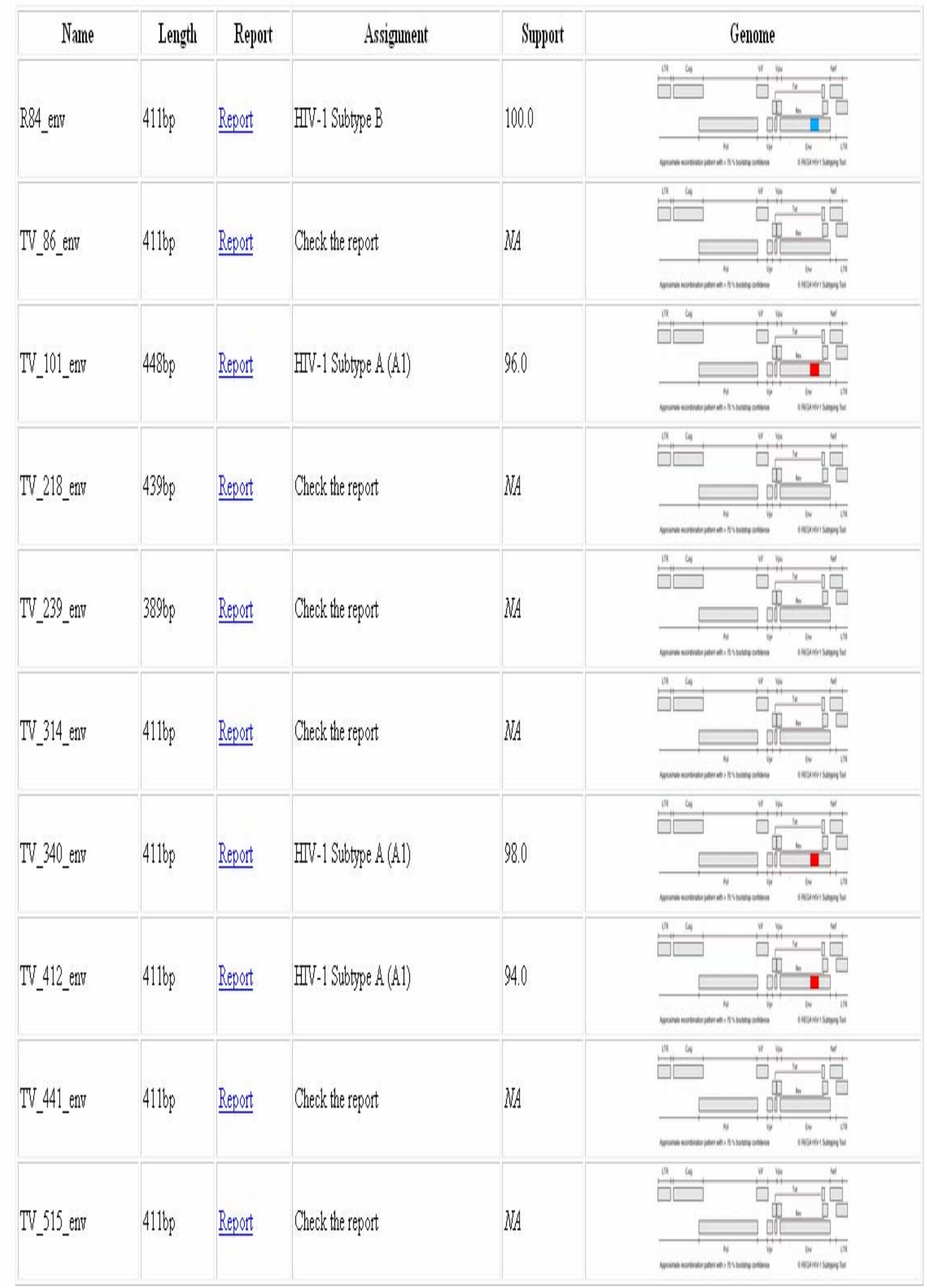

Figure 6.3: REGA subtyping results of the env gp41 sequences. The subtype and the bootstrap values are included. 
Table 6.1: jpHMM subtyping results of gag p24 subgenomic regions.

\begin{tabular}{|c|c|c|c|}
\hline Sample & Subtype & \multicolumn{2}{|c|}{ gag p24 } \\
\hline R84 & B & 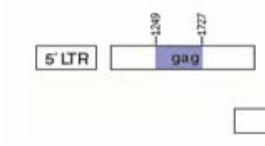 & [vitr \\
\hline TV86 & C & 5णr & viti \\
\hline TV101 & A1 & ए50 & 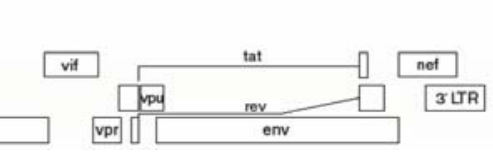 \\
\hline TV218 & C & [ITI & 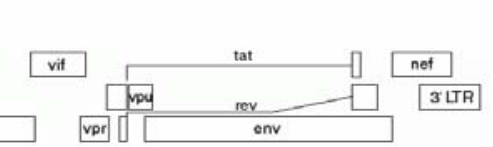 \\
\hline TV239 & A1 & 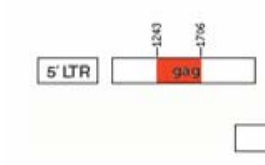 & vitt \\
\hline TV314 & A1 & इए⿴囗十 & $\sqrt{\sqrt{n+m}}$ \\
\hline TV412 & A1 & 550m & 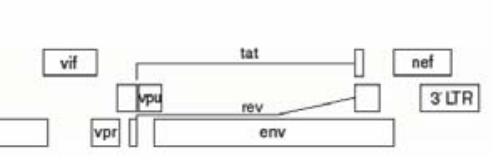 \\
\hline TV441 & C & ए5सम & [vits \\
\hline TV480 & C & 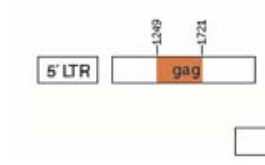 & vits \\
\hline TV515 & F1 & ELTR & vitit \\
\hline
\end{tabular}


Table 6.2: jpHMM subtyping results of pol - integrase subgenomic regions.

\begin{tabular}{|c|c|c|}
\hline Sample & Subtype & pol - integrase \\
\hline R84 & B & 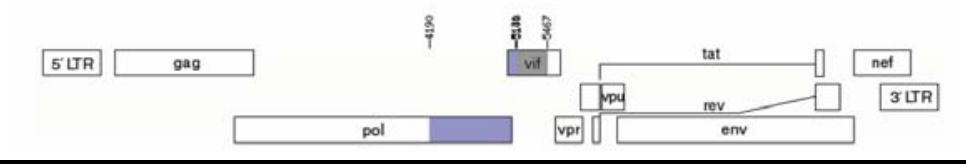 \\
\hline TV86 & C & [E[T] \\
\hline TV101 & A1 / D & 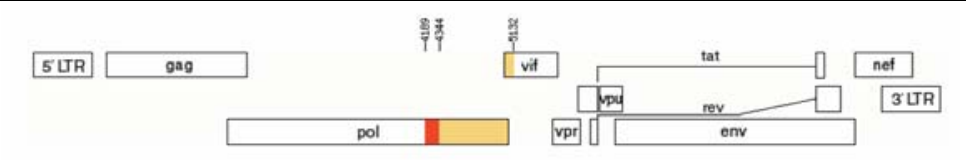 \\
\hline TV218 & C & 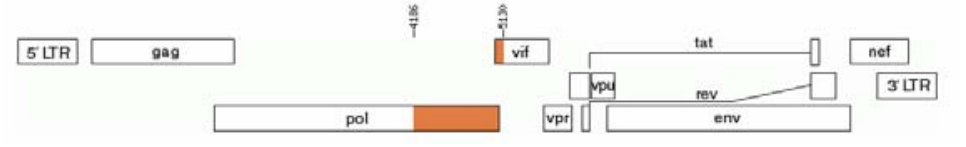 \\
\hline TV239 & A1 / H & ELTf \\
\hline TV314 & $\mathrm{A} 1 / \mathrm{J}$ & 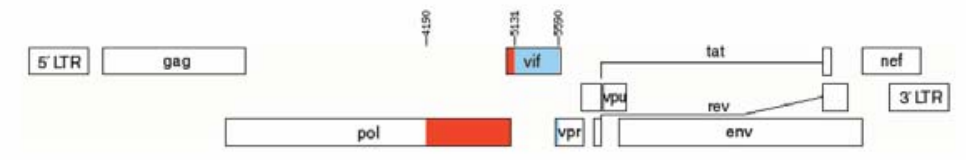 \\
\hline TV340 & G / B & 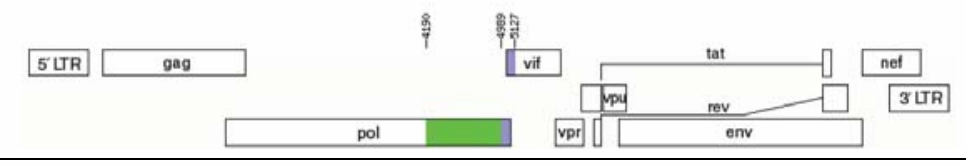 \\
\hline TV412 & $\mathrm{A} 1 / \mathrm{D} / \mathrm{J}$ & 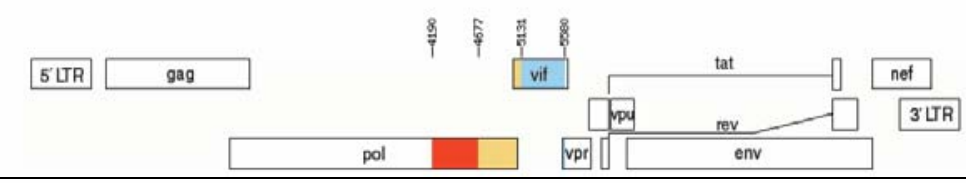 \\
\hline TV441 & C & 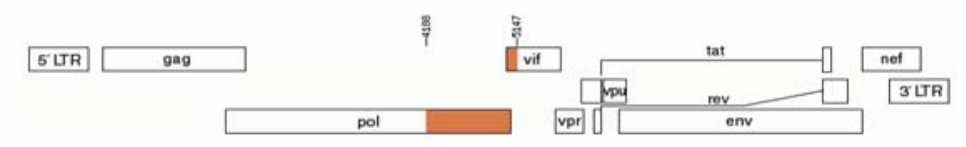 \\
\hline TV480 & $\mathrm{C} / \mathrm{J}$ & 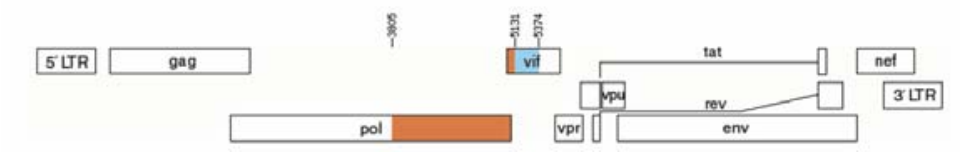 \\
\hline TV515 & F1 & [5tre $\square$ ong \\
\hline
\end{tabular}


Table 6.3: jpHMM subtyping results of env gp41 subgenomic regions.

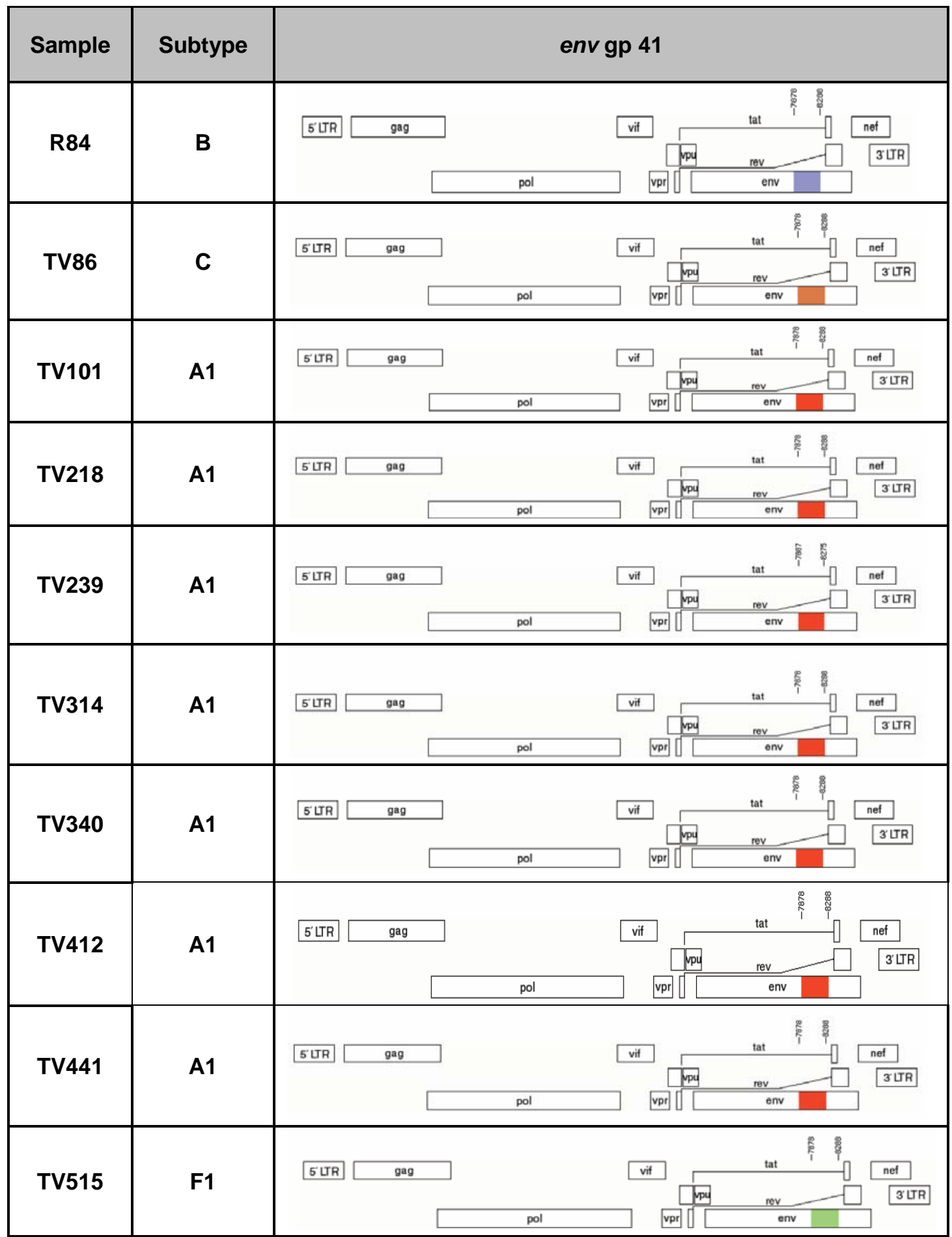




\section{Appendix C}

Table 6.4: Sequencing primers used for the characterization of R84.

\begin{tabular}{|c|c|c|c|c|}
\hline Primer & Oligo Nucleotide Sequence & HXB2 Position & $\mathbf{T}_{\mathrm{A}}$ & Ref \\
\hline $9110 \mathrm{R}$ & GCAAGAGAGACCCAGTACAG & $10166-10147$ & 50 & Personal Communication, John Hackett \\
\hline R749 LTR-gag & ATTTTGCACTATAGGGTAAT & $1181-1200$ & 45 & Personal Communication, John Hackett \\
\hline p24-1 & AGYCAAAATTAYCCYATAGT & $1174-1193$ & 45 & Swanson et al, 2003 \\
\hline p24-2 & AGRACYTTRAAYGCATGGGT & $1237-1256$ & 50 & Swanson et al, 2003 \\
\hline GagA & AGAGAACCAAGGGGAAGTGA & $1474-1493$ & 50 & Kemp et al, 1989 \\
\hline GagB & TCTCTAAAGGGTTCCTTTGG & $1654-1673$ & 45 & Kemp et al, 1989 \\
\hline p24-6 & TGTGWAGCTTGYTCRGCTC & $1703-1721$ & 50 & Swanson et al, 2003 \\
\hline cm237R2020 & GGTGGGGCTGTTGGCTCTGG & $2146-2165$ & 60 & Personal Communication, John Hackett \\
\hline cm237F2030 & GGAAACCAAAAATGATAGGGGG & $2377-2398$ & 50 & Personal Communication, John Hackett \\
\hline JA217 & СTTTTATTTTTTCTTCTGTCAATGG & $2622-2646$ & 50 & Plantier et al, 2005 \\
\hline ABB20-3F & ATCAGTACAATGTGCTTCCA & $2980-2999$ & 45 & Personal Communication, John Hackett \\
\hline RTUG F3 & GAAGCAGAATTAGAAYTGGCAGA & $3441-3463$ & 50 & Personal Communication, John Hackett \\
\hline cm237F3000 & TGTGRGTCTGTTACTATRTTTACTTC & $4023-4048$ & 50 & Personal Communication, John Hackett \\
\hline rtin seq F2 & CAACCAGAYARRAGTGAATCAGA & $4074-4096$ & 50 & Personal Communication, S Engelbrecht \\
\hline poli7 & AACAAGTAGATAAATTAGTCAGT & $4186-4208$ & 45 & Swanson et al, 2003 \\
\hline PPF17 & AATTGGAGAGCAATGGCTAGTGA & $4281-4303$ & 50 & Swanson et al, 2003 \\
\hline ppr15 & ССTTCTAAATGTGTACAATC & $4419-4438$ & 45 & Personal Communication, John Hackett \\
\hline PPF2b & GCAGTCCATGTAGCCAGTGG & $4455-4474$ & 50 & Personal Communication, John Hackett \\
\hline FGF46 & GCATTCCCTACAATCCCCAAAG & $4648-4669$ & 55 & Fong et al, 1996 \\
\hline
\end{tabular}

Key: $T_{A}$, (Annealing temperature), and Ref (Reference) 
Table 6.4 continued: Sequencing primers used for the characterization of R84.

\begin{tabular}{|l|l|c|c|l|}
\hline \multicolumn{1}{|c|}{ Primer } & \multicolumn{1}{|c|}{ Oligo Nucleotide Sequence } & HXB2 Position & T $_{\text {A }}$ & \multicolumn{1}{c|}{ Ref } \\
\hline poli10b & TATTCATAGATTCYACTACTCCTTG & $4671-4695$ & 50 & Personal Communication, John Hackett \\
\hline poli2 & TAAARACARYAGTACWAATGGCA & $4744-4766$ & 50 & Personal Communication, John Hackett \\
\hline PPR5b & ACTACTGCCCCTTCACCTTTCCA & $4956-4978$ & 55 & Personal Communication, John Hackett \\
\hline poli6 & ATACATATGRTGTTTTACTAARCT & $5107-5130$ & 45 & Personal Communication, John Hackett \\
\hline PPR6 & CCTGCCATCTGTTTTCCATA & $5040-5059$ & 45 & Personal Communication, John Hackett \\
\hline 55env272R & TCTGGGGCTTGTTCCATCTATCTT & $5552-5575$ & 55 & Personal Communication, John Hackett \\
\hline 55env-5820R & ATCAAAGTCCCCCATCTCCACAAG & $6251-6273$ & 55 & Personal Communication, John Hackett \\
\hline 20env5'2287F & CTTTGAGCCCATTCCCATACATTA & $6851-6874$ & 50 & Personal Communication, John Hackett \\
\hline 55env222R & AATCGCAAAACCAGCTGGAGCAC & $6877-6899$ & 55 & Personal Communication, John Hackett \\
\hline ES7X & CTGTTAAATGGCAGTCTAGC & $7002-7021$ & 55 & Bachmann et al, 1994 \\
\hline ES125 & CAATTCTGGGTCCCCTCCTGAG & $7316-7338$ & 60 & Bachmann et al, 1994 \\
\hline ES8X & CACTTCTCCAATTGTCCCTCA & $7648-7668$ & 55 & Bachmann et al, 1994 \\
\hline SK68 & AGCAGCAGGAGCACTATGG & $7796-7815$ & 55 & Ou et al, 1988 \\
\hline env 27F & CTGGYATAGTGCARCARCA & $7861-7879$ & 50 & Swanson et al, 2003 \\
\hline 7496F & CCTKGCYCTGGAAAGATACCTA & $7964-7985$ & 45 & Personal Communication, John Hackett \\
\hline 7542F.1 & TGGGGCTGCTCTGGAAACT & $8010-8029$ & 55 & Personal Communication, John Hackett \\
\hline LP7728R & CCACTTGTCCAATGCCAATAAGTCTTT & $8222-8195$ & 55 & Personal Communication, John Hackett \\
\hline Menv 19R & AARCCTCCTACTATCATTATRA & $8278-8299$ & 45 & Swanson et al, 2003 \\
\hline GP41R1 & AACGACAAAGGTGAGTATCCCTGCCTA & $8347-8374$ & 60 & Pieniazek et al, 1998 \\
\hline 20LTR-3825F & TGGGTGGCAAGTGGTCAAAAGTA & $8798-8821$ & 55 & Personal Communication, John Hackett \\
\hline 20env 4038R & GTACCTGCGGCTGACTGGA & $9000-9019$ & 55 & Personal Communication, John Hackett \\
\hline Key: TA, (Anneam & & & & \\
\hline
\end{tabular}

Key: $\mathrm{T}_{\mathrm{A}}$, (Annealing temperature), and Ref (Reference) 
Table 6.5: Sequencing primers used for the sequencing of sample TV239

\begin{tabular}{|c|c|c|c|c|}
\hline Primer & Oligo Nucleotide Sequence & $\begin{array}{c}\text { HXB2 } \\
\text { Position }\end{array}$ & $\mathbf{T}_{\mathbf{A}}$ & Ref \\
\hline env $\mathrm{N}$ & CTGCCAATCAGGGAAGTAGCCTTGTGT & $60-86$ & 55 & Derdeyn et al, 2004 \\
\hline $\mathrm{p} 24-2$ & AGRACYTTRAAYGCATGGGT & $1237-1245$ & 50 & Swanson et al, 2003 \\
\hline $\mathrm{p} 24-6$ & TGTGWAGCTTGYTCRGCTC & $1703-1721$ & 50 & Swanson et al, 2003 \\
\hline F1432 & AGGCAATGAGTCAAGTACAACA & $1883-1904$ & 50 & Personal Communication, John Hackett \\
\hline $\mathrm{cm} 237 \mathrm{R} 2020$ & GGTGGGGCTGTTGGCTCTGG & $2146-2165$ & 60 & Personal Communication, John Hackett \\
\hline cm237F2030 & GGAAACCAAAAATGATAGGGGG & $2377-2398$ & 50 & Personal Communication, John Hackett \\
\hline JA217 & CTTTTATTTTTTCTTCTGTCAATGG & $2622-2646$ & 50 & Plantier et al, 2005 \\
\hline ABB20-3F & ATCAGTACAATGTGCTTCCA & $2980-2999$ & 45 & Personal Communication, John Hackett \\
\hline ABB20-11R & TATGTCCATTGGTCTTGCCC & $3546-3565$ & 50 & Personal Communication, John Hackett \\
\hline RTUG F3 & GAAGCAGAATTAGAAYTGGCAGA & $3441-3463$ & 50 & Personal Communication, John Hackett \\
\hline $\mathrm{cm} 237 \mathrm{~F} 3000$ & TGTGRGTCTGTTACTATRTTTACTTC & $4023-4048$ & 50 & Personal Communication, John Hackett \\
\hline rtin seq F2 & CAACCAGAYARRAGTGAATCAGA & $4074-4096$ & 50 & Personal Communication, S Engelbrecht \\
\hline poli7 & AACAAGTAGATAAATTAGTCAGT & $4186-4208$ & 45 & Swanson et al, 2003 \\
\hline PPF17 & AATTGGAGAGCAATGGCTAGTGA & $4281-4303$ & 50 & Personal Communication, John Hackett \\
\hline ppr15 & СCTTCTAAATGTGTACAATC & $4419-4438$ & 45 & Personal Communication, John Hackett \\
\hline PPF2b & GCAGTCCATGTAGCCAGTGG & $4455-4474$ & 50 & Personal Communication, John Hackett \\
\hline FGF46 & GCATTCCCTACAATCCCCAAAG & $4648-4669$ & 55 & Fong et al, 1996 \\
\hline poli10b & TATTCATAGATTCYACTACTCCTTG & $4671-4695$ & 50 & Personal Communication, John Hackett \\
\hline poli2 & TAAARACARYAGTACWAATGGCA & $4744-4766$ & 50 & Swanson et al, 2003 \\
\hline PPR6 & СCTGCCATCTGTTTTCCATA & $5040-5059$ & 45 & Personal Communication, John Hackett \\
\hline 55env272R & TCTGGGGCTTGTTCCATCTATCTT & $5552-5575$ & 55 & Personal Communication, John Hackett \\
\hline
\end{tabular}

Key: $T_{A}$, (Annealing temperature), and Ref (Reference) 
Table 6.5 continued: Sequencing primers used for the sequencing of sample TV239

\begin{tabular}{|l|l|c|c|l|}
\hline \multicolumn{1}{|c|}{ Primer } & \multicolumn{1}{|c|}{ Oligo Nucleotide Sequence } & $\begin{array}{c}\text { HXB2 } \\
\text { Position }\end{array}$ & $\mathbf{T}_{\text {A }}$ & \multicolumn{1}{c|}{ Ref } \\
\hline 55env-5521R & GCTTCCGCTTCTTCCTGCCATAG & $5968-5990$ & 55 & Personal Communication, John Hackett \\
\hline 20env5'2287F & CTTTGAGCCCATTCCCATACATTA & $6851-6874$ & 50 & Personal Communication, John Hackett \\
\hline 55env222R & AATCGCAAAACCAGCTGGAGCAC & $6877-6899$ & 55 & Personal Communication, John Hackett \\
\hline ES7X & CTGTTAAATGGCAGTCTAGC & $7002-7021$ & 55 & Bachmann et al, 1994 \\
\hline ES8X & CACTTCTCCAATTGTCCCTCA & $7648-7668$ & 55 & Bachmann et al, 1994 \\
\hline SK68 & AGCAGCAGGAAGCACTATGG & $7796-7815$ & 55 & Ou et al, 1988 \\
\hline ED12 & AGTGCTTCCTGCTGCTCCCAAGAACCCA & $7782-7811$ & 60 & Delwart et al, 1993 \\
\hline env 27F & CTGGYATAGTGCARCARCA & $7861-7879$ & 50 & Personal Communication, John Hackett \\
\hline LP7725R & GTCCAATGCCAATAAGTCTTGTTC & $8193-8216$ & 50 & Personal Communication, John Hackett \\
\hline Menv 19R & AARCCTCCTACTATCATTATRA & $8278-8299$ & 45 & Swanson et al, 2003 \\
\hline 69env-3848F & TGCTGCGAGGGGTGTGGACTT & $8555-8576$ & 60 & Personal Communication, John Hackett \\
\hline 20env 4038R & GTACCTGCGGCCTGACTGGA & $9000-9019$ & 55 & Personal Communication, John Hackett \\
\hline
\end{tabular}

Key: $T_{A}$, (Annealing temperature), and Ref (Reference) 
Table 6.6: Sequencing primers used for the sequencing of TV314.

\begin{tabular}{|c|c|c|c|c|}
\hline Primer & Oligo Nucleotide Sequence & $\begin{array}{c}\text { HXB2 } \\
\text { Position }\end{array}$ & $\mathbf{T}_{\mathbf{A}}$ & Ref \\
\hline $\mathrm{p} 24-6$ & TGTGWAGCTTGYTCRGCTC & $1703-1721$ & 50 & Swanson et al, 2003 \\
\hline F1432 & AGGCAATGAGTCAAGTACAACA & $1883-1904$ & 50 & Personal Communication, John Hackett \\
\hline $\mathrm{cm} 237 \mathrm{R} 2020$ & GGTGGGGCTGTTGGCTCTGG & $2146-2165$ & 60 & Personal Communication, John Hackett \\
\hline cm237F2030 & GGAAACCAAAAATGATAGGGGG & $2377-2398$ & 50 & Personal Communication, John Hackett \\
\hline JA217 & СTTTTATTTTTTCTTCTGTCAATGG & $2622-2646$ & 50 & Plantier et al, 2005 \\
\hline ABB20-3F & ATCAGTACAATGTGCTTCCA & $2980-2999$ & 45 & Personal Communication, John Hackett \\
\hline ABB20-11R & TATGTCCATTGGTCTTGCCC & $3546-3565$ & 50 & Personal Communication, John Hackett \\
\hline RTUG F3 & GAAGCAGAATTAGAAYTGGCAGA & $3441-3463$ & 50 & Personal Communication, John Hackett \\
\hline $\mathrm{cm} 237 \mathrm{~F} 3000$ & TGTGRGTCTGTTACTATRTTTACTTC & $4023-4048$ & 50 & Personal Communication, John Hackett \\
\hline rtin seq F2 & CAACCAGAYARRAGTGAATCAGA & $4074-4096$ & 50 & Personal Communication, S Engelbrecht \\
\hline PPF2b & GCAGTCCATGTAGCCAGTGG & $4455-4474$ & 50 & Personal Communication, John Hackett \\
\hline poli10b & TATTCATAGATTCYACTACTCCTTG & $4671-4695$ & 50 & Personal Communication, John Hackett \\
\hline poli2 & TAAARACARYAGTACWAATGGCA & $4744-4766$ & 50 & Swanson et al, 2003 \\
\hline PPR6 & СCTGCCATCTGTTTTCCATA & $5040-5059$ & 45 & Personal Communication, John Hackett \\
\hline 55 env4F & GGTGGTGGGGCCTCCATAGAAT & $5284-5305$ & 55 & Personal Communication, John Hackett \\
\hline 55 env272R & TCTGGGGCTTGTTCCATCTATCTT & $5552-5575$ & 55 & Personal Communication, John Hackett \\
\hline $20 \mathrm{env}-1301 \mathrm{~F}$ & ACTATGGGGTACCGGTGTGGAGA & $6340-6362$ & 55 & Personal Communication, John Hackett \\
\hline 55 env222R & AATCGCAAAACCAGCTGGAGCAC & $6877-6899$ & 55 & Personal Communication, John Hackett \\
\hline ES7X & CTGTTAAATGGCAGTCTAGC & $7002-7021$ & 55 & Bachmann et al, 1994 \\
\hline E120 & GTAGAAATTAATTGTACAAGACCC & $7098-7121$ & 45 & Bachmann et al, 1994 \\
\hline
\end{tabular}

Key: $\mathrm{T}_{\mathrm{A}}$ (Annealing temperature), $\mathrm{F}$ (Forward primer) and $\mathrm{R}$ (Reverse primer) 
Table 6.6 continued: Sequencing primers used for the sequencing of TV314.

\begin{tabular}{|l|l|c|c|l|}
\hline \multicolumn{1}{|c|}{ Primer } & \multicolumn{1}{|c|}{ Oligo Nucleotide Sequence } & $\begin{array}{c}\text { HXB2 } \\
\text { Position }\end{array}$ & $\mathbf{T}_{\mathbf{A}}$ & \multicolumn{1}{c|}{ Ref } \\
\hline SK68 & AGCAGCAGGAAGCACTATGG & $7796-7815$ & 55 & Ou et al, 1988 \\
\hline 7542F.1 & TGGGGCTGCTCTGGAAAACT & $8010-8029$ & 55 & Personal Communication, John Hackett \\
\hline LP7725R & GTCCAATGCCAATAAGTCTTGTTC & $8193-8216$ & 50 & Personal Communication, John Hackett \\
\hline 20env-3481R & CAGGCAAGCGCGAAGAATCC & $8475-8494$ & 55 & Personal Communication, John Hackett \\
\hline 69env-3848F & TGCTGCGAGGGGTGTGGAACTT & $8555-8576$ & 60 & Personal Communication, John Hackett \\
\hline 20LTR-3825F & TGGGTGGCAAGTGGTCAAAAAGTA & $8798-8821$ & 55 & Personal Communication, John Hackett \\
\hline 20env 4038R & GTACCTGCGGCCTGACTGGA & $9000-9019$ & 55 & Personal Communication, John Hackett \\
\hline ABB55-4-1186 & CCAGGGCCAGGGGTTAGAT & $9181-9199$ & 55 & Personal Communication, John Hackett \\
\hline
\end{tabular}

Key: $\mathrm{T}_{\mathrm{A}}$ (Annealing temperature), $\mathrm{F}$ (Forward primer) and $\mathrm{R}$ (Reverse primer) 
Table 6.7: Sequencing primers used for the sequencing of TV 412.

\begin{tabular}{|c|c|c|c|c|}
\hline Primer & Oligo Nucleotide Sequence & $\begin{array}{c}\text { HXB2 } \\
\text { Position }\end{array}$ & $\mathbf{T}_{\mathbf{A}}$ & Ref \\
\hline $\mathrm{p} 24-2$ & AGRACYTTRAAYGCATGGGT & $1237-1256$ & 50 & Swanson et al, 2003 \\
\hline p24-6 & TGTGWAGCTTGYTCRGCTC & $1703-1721$ & 50 & Swanson et al, 2003 \\
\hline cm237F2030 & GGAAACCAAAAATGATAGGGGG & $2377-2398$ & 50 & Personal Communication, John Hackett \\
\hline JA217 & CTTTTATTTTTTCTTCTGTCAATGG & $2622-2646$ & 50 & Plantier et al, 2005 \\
\hline ABB20-3F & ATCAGTACAATGTGCTTCCA & $2980-2999$ & 45 & Personal Communication, John Hackett \\
\hline ABB20-11R & TATGTCCATTGGTCTTGCCC & $3546-3565$ & 50 & Personal Communication, John Hackett \\
\hline RTUG F3 & GAAGCAGAATTAGAAYTGGCAGA & $3441-3463$ & 50 & Personal Communication, John Hackett \\
\hline $\mathrm{cm} 237 \mathrm{~F} 3000$ & TGTGRGTCTGTTACTATRTTTACTTC & $4023-4048$ & 50 & Personal Communication, John Hackett \\
\hline rtin seq F2 & CAACCAGAYARRAGTGAATCAGA & $4074-4096$ & 50 & Personal Communication, S Engelbrecht \\
\hline PPF17 & AATTGGAGAGCAATGGCTAGTGA & $4281-4303$ & 50 & Personal Communication, John Hackett \\
\hline ppr15 & CCTTCTAAATGTGTACAATC & $4419-4438$ & 45 & Personal Communication, John Hackett \\
\hline PPF2b & GCAGTCCATGTAGCCAGTGG & $4455-4474$ & 50 & Personal Communication, John Hackett \\
\hline poli10b & TATTCATAGATTCYACTACTCCTTG & $4671-4695$ & 50 & Swanson et al, 2003 \\
\hline poli2 & TAAARACARYAGTACWAATGGCA & $4744-4766$ & 50 & Personal Communication, John Hackett \\
\hline PPR6 & СCTGCCATCTGTTTTCCATA & $5040-5059$ & 45 & Personal Communication, John Hackett \\
\hline 55env272R & TCTGGGGCTTGTTCCATCTATCTT & $5552-5575$ & 55 & Personal Communication, John Hackett \\
\hline 55env-5820R & ATCAAAGTCCCCCATCTCCACAAG & $6251-6273$ & 55 & Personal Communication, John Hackett \\
\hline 20 env-1301F & ACTATGGGGTACCGGTGTGGAGA & $6340-6362$ & 55 & Personal Communication, John Hackett \\
\hline 20env5'2287F & CTTTGAGCCCATTCCCATACATTA & $6851-6874$ & 50 & Personal Communication, John Hackett \\
\hline
\end{tabular}

Key: $T_{A}$ (Annealing temperature), and Ref (Reference) 
Table 6.7 continued: Sequencing primers used for the sequencing of TV 412.

\begin{tabular}{|l|l|c|c|l|}
\hline \multicolumn{1}{|c|}{ Primer } & \multicolumn{1}{|c|}{ Oligo Nucleotide Sequence } & $\begin{array}{c}\text { HXB2 } \\
\text { Position }\end{array}$ & \multicolumn{1}{c|}{ TA $_{\text {A }}$} & \multicolumn{1}{c|}{ Ref } \\
\hline 55env222R & AATCGCAAAACCAGCTGGAGCAC & $6877-6899$ & 55 & Personal Communication, John Hackett \\
\hline ES7X & CTGTTAAATGGCAGTCTAGC & $7002-7021$ & 55 & Bachmann et al, 1994 \\
\hline 20env-2513F & GCAGAAAGTAGGGCAAGCAATGTA & $7505-7528$ & 55 & Personal Communication, John Hackett \\
\hline SK68 & AGCAGCAGGAAGCACTATGG & $7796-7815$ & 55 & Ou et al, 1988 \\
\hline LP7725R & GTCCAATGCCAATAAGTCTTGTTC & $8193-8216$ & 50 & Personal Communication, John Hackett \\
\hline GP41R1 & AACGACAAAGGTGAGTATCCCTGCCTAA & $8374-8374$ & 60 & Pieniazek et al, 1998 \\
\hline JH38 & GGTGARTATCCCTKCCTAAC & $8346-8365$ & 45 & Swanson et al, 2003 \\
\hline GP47R2 & TTAAACCTATCAAGCCTCCTACTATCATTA & $8281-8310$ & 50 & Pieniazek et al, 1998 \\
\hline env-end & CTTTTTGACCACTTGCCACCCAT & $8797-8819$ & 55 & Personal Communication, S Engelbrecht \\
\hline Key: TA
\end{tabular}

Key: $\mathrm{T}_{\mathrm{A}}$ (Annealing temperature), and Ref (Reference) 


\section{Appendix D}

\subsection{Gene Cutter Results of NFLG's}

Sample R84 from 601 - 9514 (coordinates relative to HXB2)

Nucleotide and Amino Acid composition.

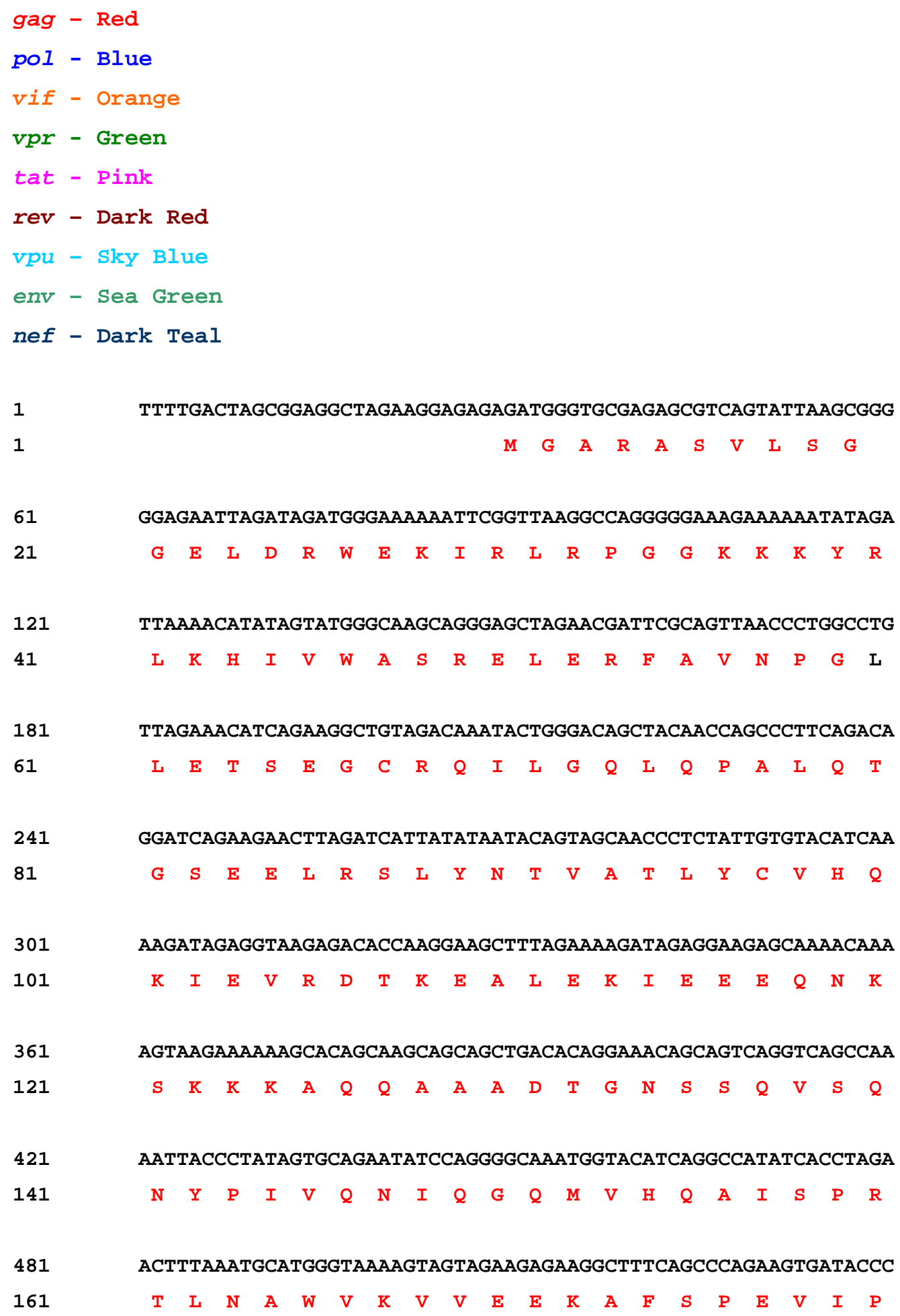




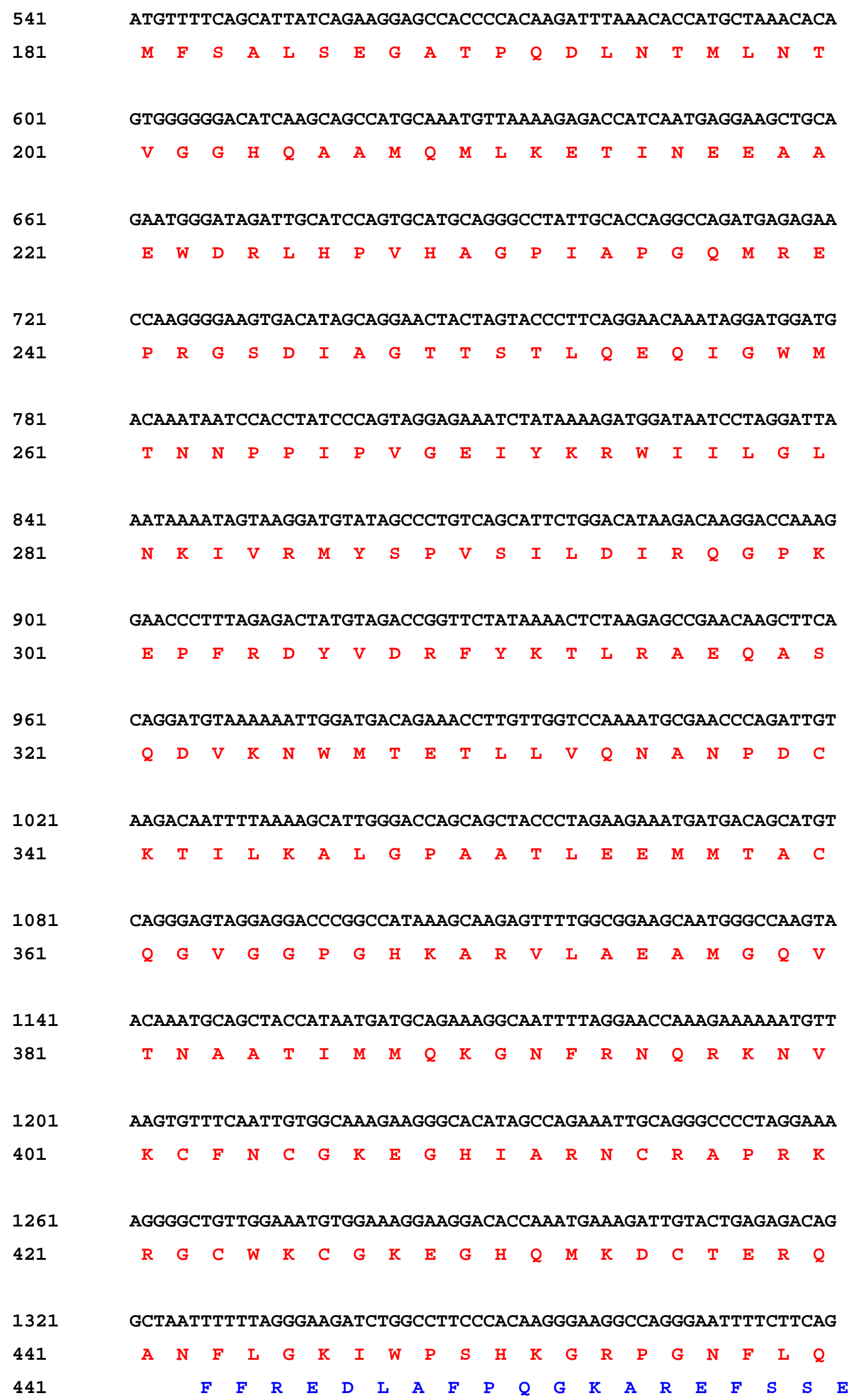


AGCAGACCAGAGCCAACAGCCCCACCAGAAGAGAGCTTCAGGTTTGGGGAAGAGACAACA

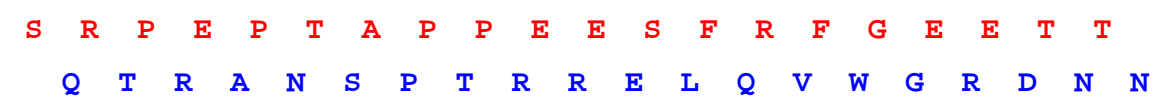
ACTCCCTCTCAGAAGCAGgAGCCGATAGACAAGGAACTATATCCTTTAGCTTCCCTCAGA $\begin{array}{lllllllllllllllllllll}T & P & S & Q & K & Q & E & P & I & D & K & E & L & Y & P & L & A & S & L & R\end{array}$ $\begin{array}{llllllllllllllllllll}S & L & S & E & A & G & A & D & R & Q & G & T & I & S & F & S & F & P & Q & I\end{array}$ TCACTCTTTGGCAACGACCCCTCGTCACAATAAAGATAGGGGGGCACCTAAAGGAAGCTC

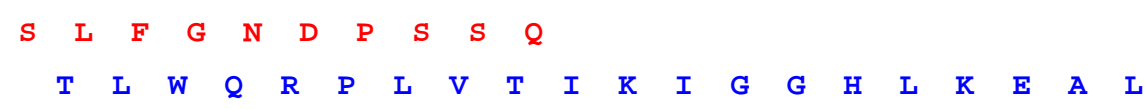




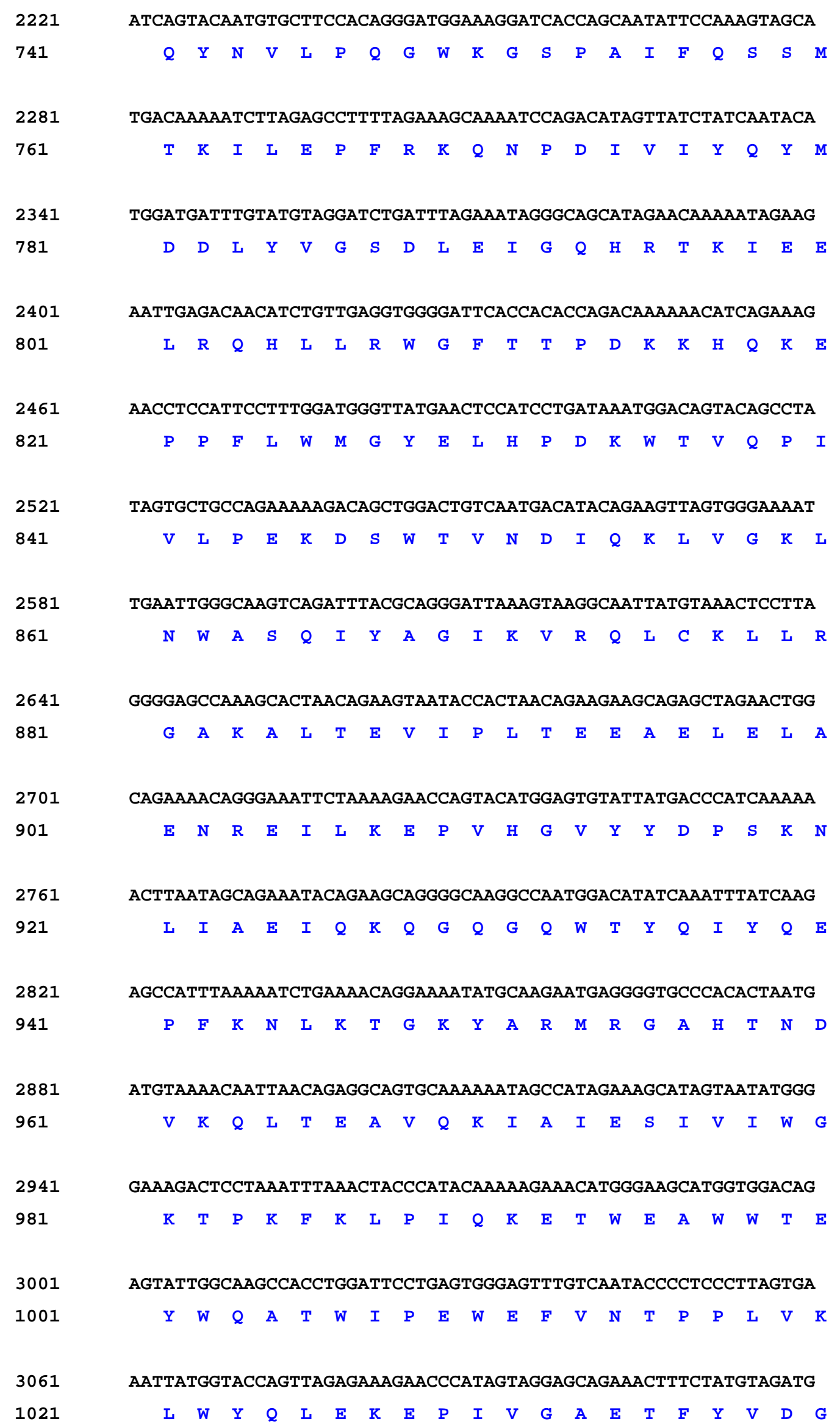




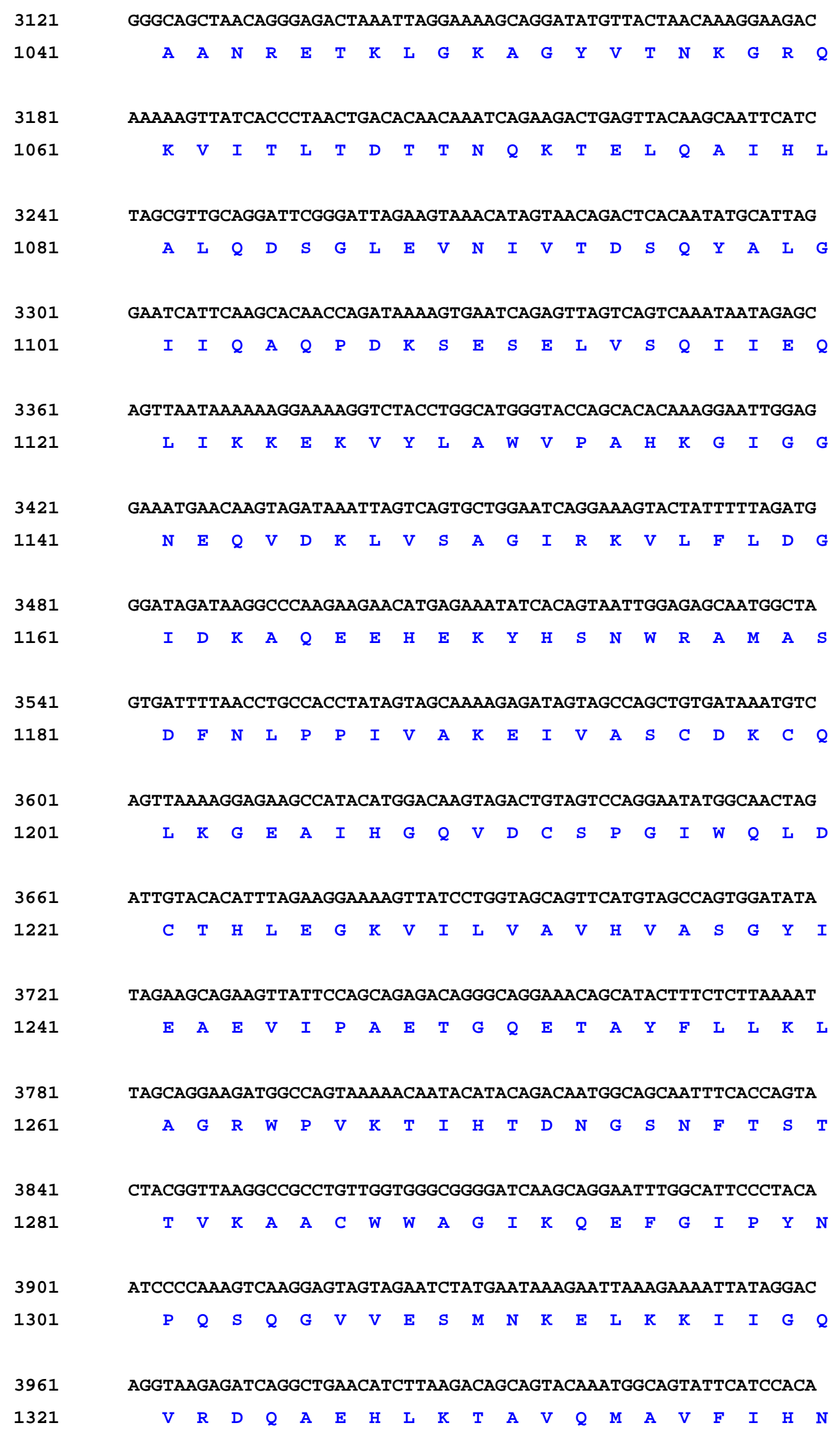




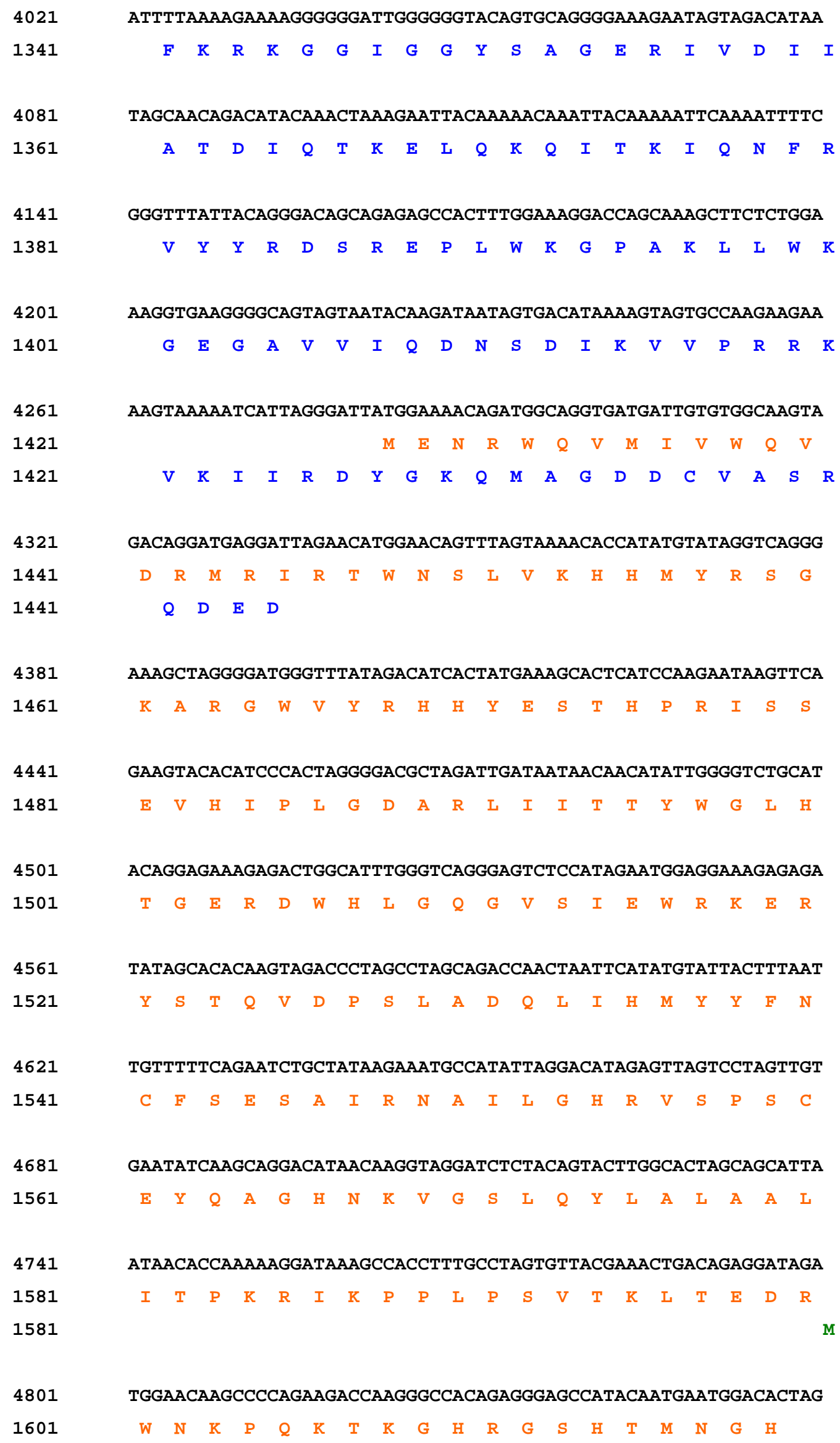




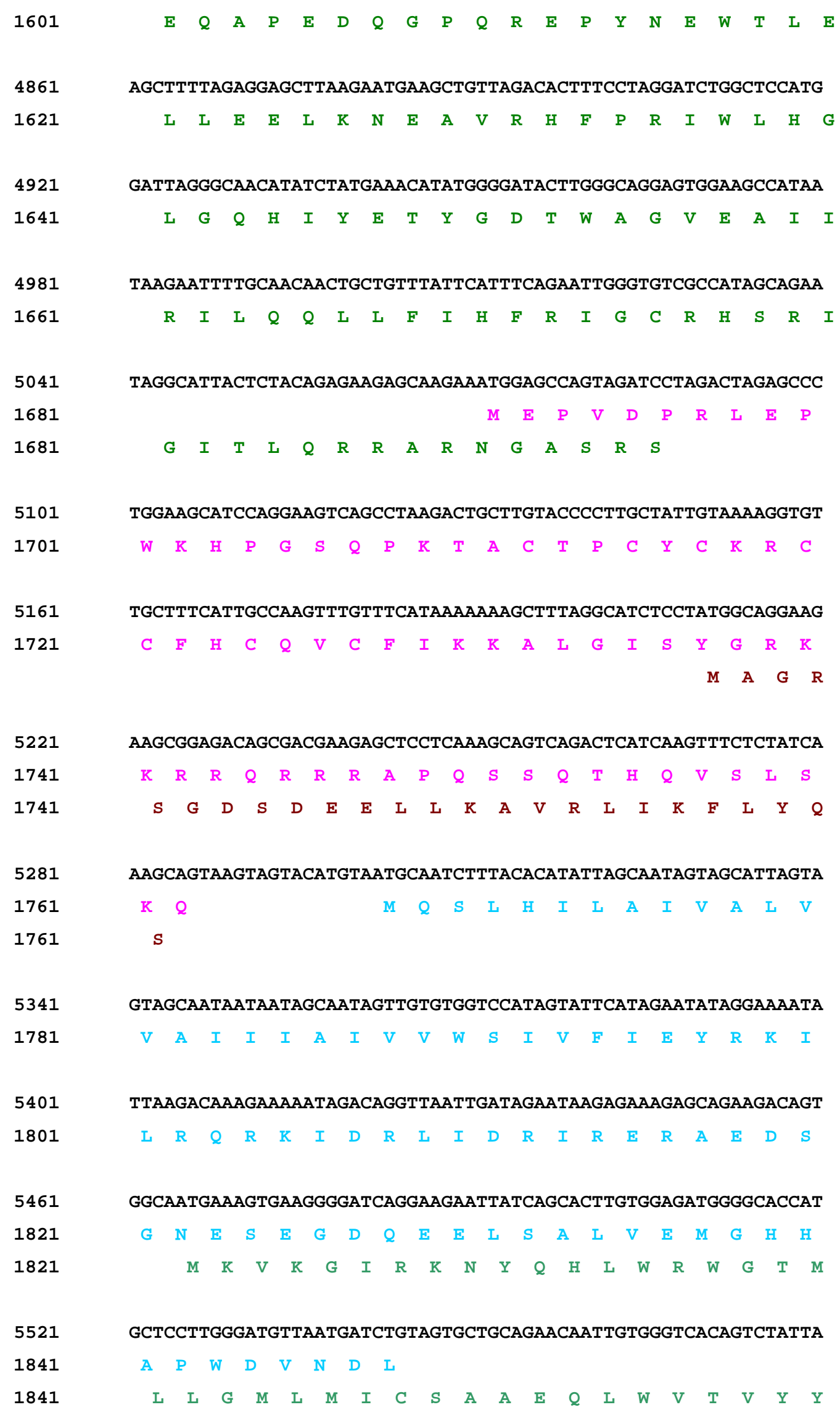


TAGAGTTAGgCAGgGATACTCACCATTATCGTTGCAGACCCGCCTGCCAGCAGCCTCGAG

$$
\begin{array}{llllllllllllllllllllllllllllll}
R & V & R & Q & G & Y & S & P & L & S & L & Q & T & R & L & P & A & A & S & R \\
& & & & & & P & A & C & Q & Q & P & R & G
\end{array}
$$

7621

GGGACCCGACAGGCCCGAAGGATCGAAGAAGAAGGTGGAGAGAGAGACAGAGACAGATC

CGGTGGATTAGTGAACGGATTCTTAGCACTTATCTGGATCGACCTACGGAGCCTGTGCCT

GGGACGCAGGGGGTGGGACCCCTCAAATATTGGTGGAATCTCCTACAGTATTGGAGTCA

GGAACTAAAGAATAGTGCTATTAGCTTGCTCAATGCCACAGCCATAGCAGTAGCTGAGGG 


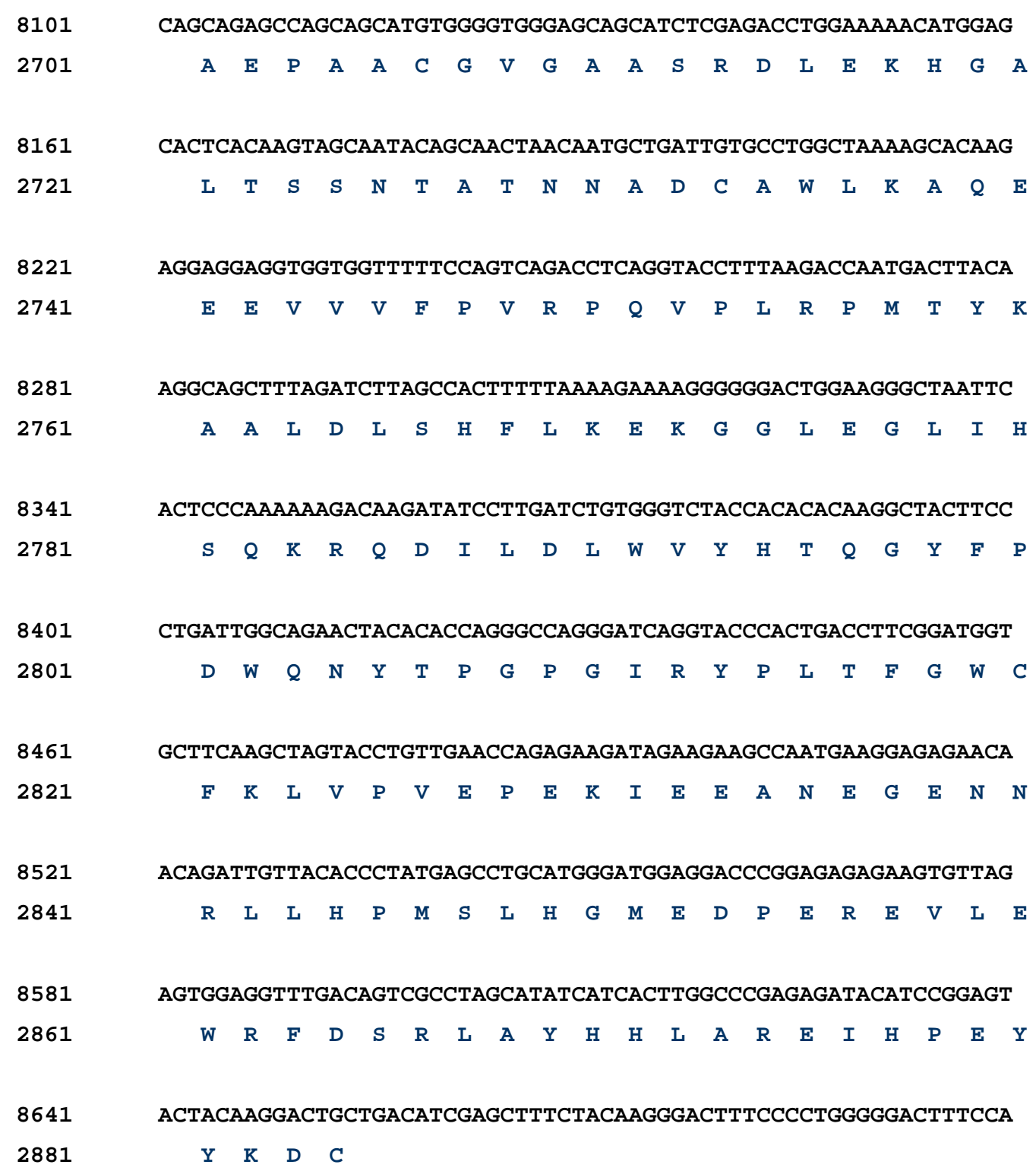


Sample TV 239 gag-pol from 1245 - 5534 (coordinates relative to HSB2) Nucleotide and Amino Acid composition

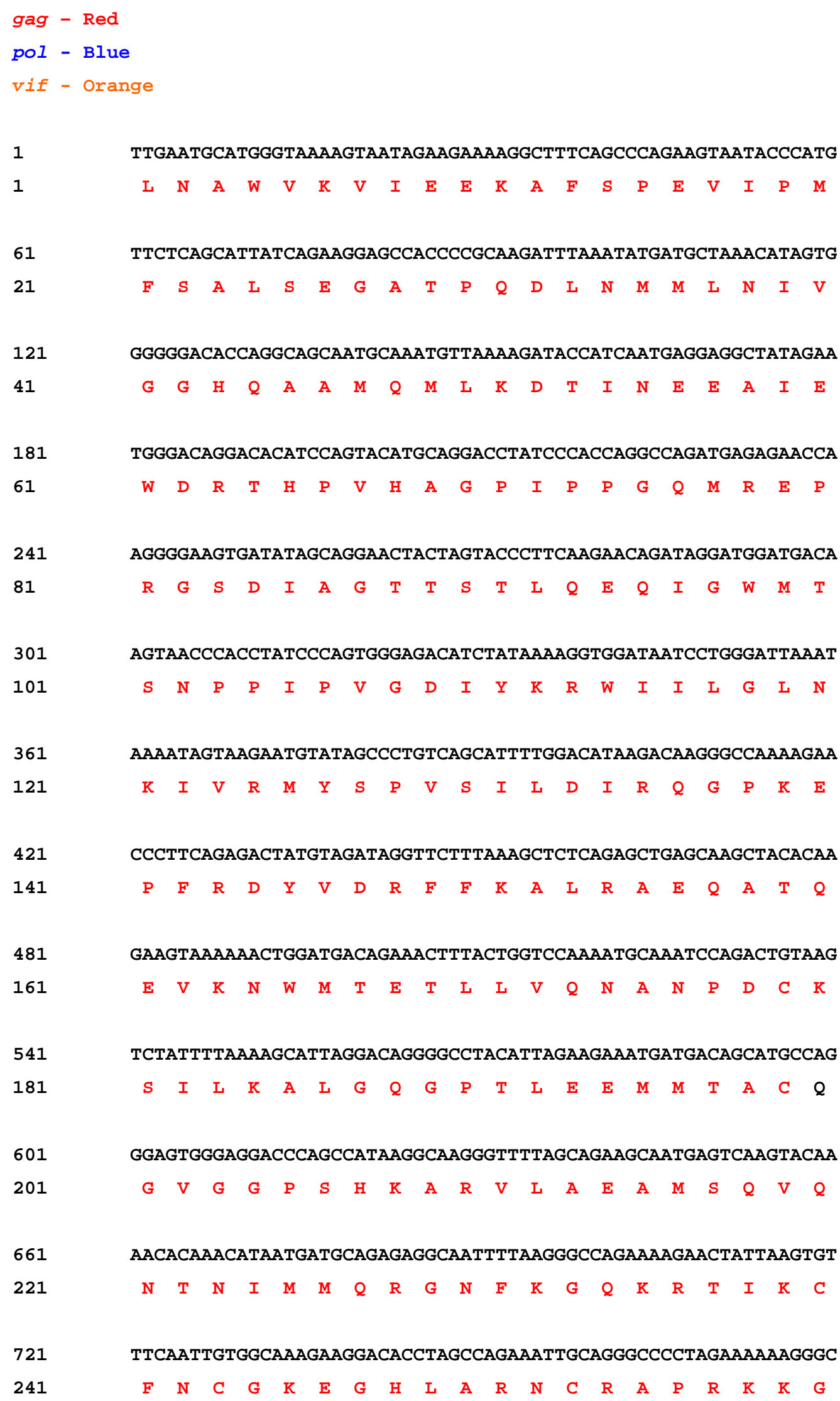




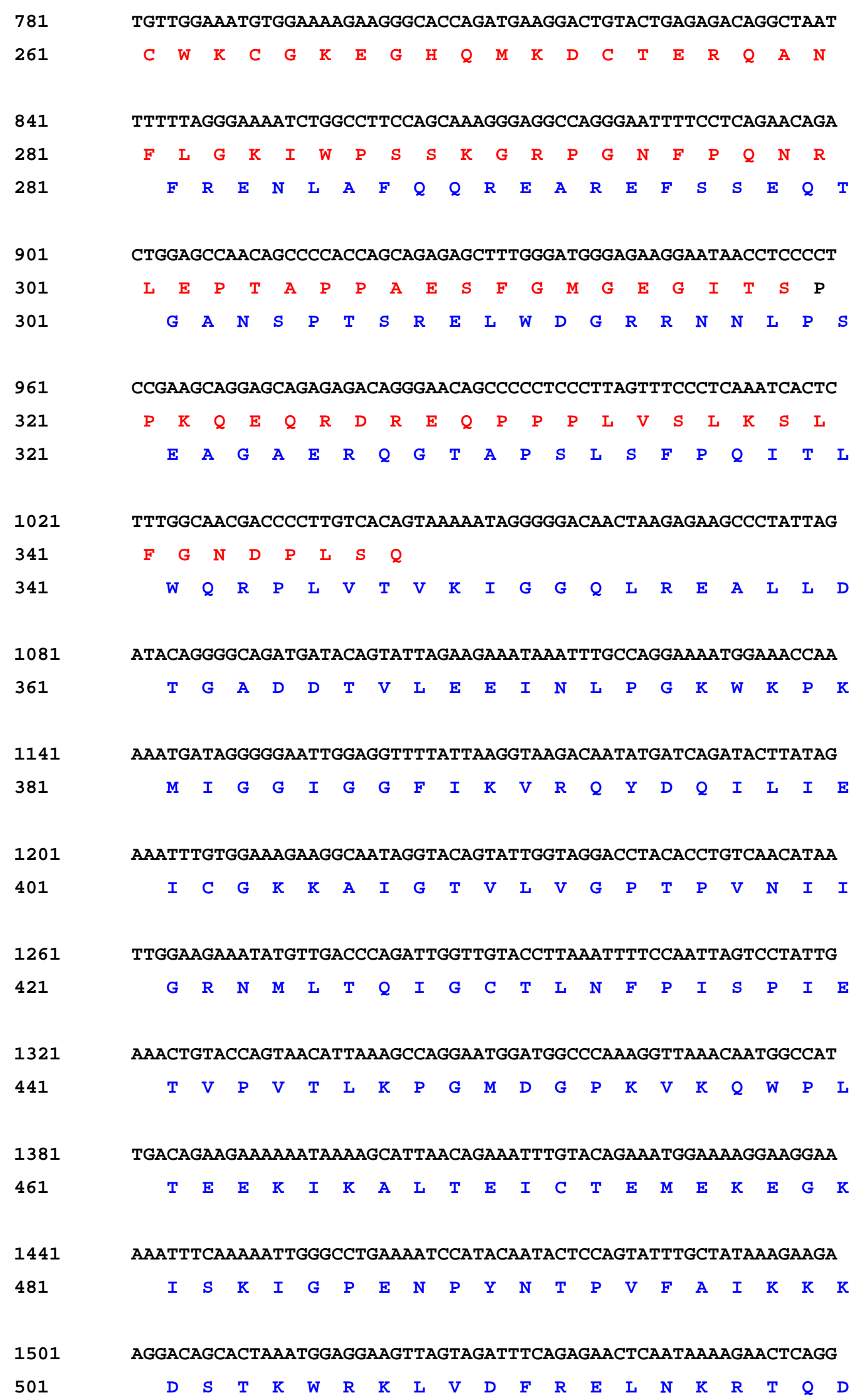


CACATCTAGAAGgAAAGTAATCATAGTAGCAGTCCATGTAGCCAGTGGCTATATAGAGG

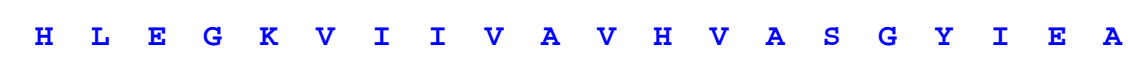
CAGAAGTTATCCCAGCAGAAACAGGACAGGAGGCAGCATACTTTCTGTTAAAATTAGCAG

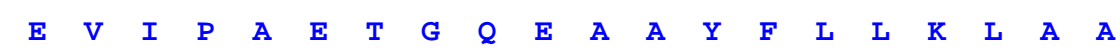
CAAGATGGCCAGTAAAGGTAATACACACAGACAATGGCAGTAATTTCACCAGTGCCGCAG $\begin{array}{lllllllllllllllllllll}R & \text { W } & \text { P } & \text { V } & \text { K } & \text { V } & \text { I } & \text { H } & \text { T } & \text { D } & \text { N } & \text { G } & \text { S } & \text { N } & \text { F } & \text { T } & \text { S } & \text { A } & \text { A } & \text { V }\end{array}$ 
TTAAAGCAGCCTGTTGGTGGGCAAATATCCAACAGGAATTTGGAATTCCCTACAATCCCC

AAATCATTAGGGACTATGGAAAACAGATGGCAGGTGATGATTGTGTGGCAGGTAGACAGG

CACATCCCACTAGgGGATGCTAGATTAGTAGTAAAAACATATTGGgGTCTGCAGACAGGA

GAAAAAGACTGGCAATTGGGTCATGGGGTCTCCATAGAATGGAGACAGAACAGATATGTT 
4261

CCAAGAAAGCCAAAGCCACCTGCCCAG

1421

$\begin{array}{lllllll}P & R & K & P & K & P & P\end{array}$


Sample TV 239 env-nef from 6195-9146 (coordinates relative to HXB2) Nucleotide and amino acid composition.

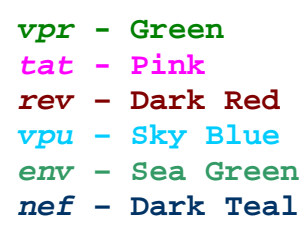

1

1

1

61

21

21

121

41

181

61

241

81

301

101

361

121

421

141

481

161

541

181

601

201

661

221

GAATTAGgGAAGAGCAGAAGACAGTGGCAATGAGAGTGATGGGGATACAGAGGAATTGT

$\begin{array}{llllllllllllllllllll}M & R & V & M & G & I & Q & R & N & C\end{array}$ CAACAATGGTGGATATGgGGCATCTTAGGCTTTTGGATGATATATAATGGGATGGgGGCG

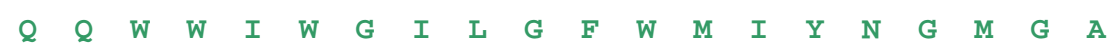

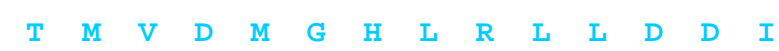

GGCTTGTGGGTCACGGTCTATTATGGAGTACCTGTGTGGAAAGACGCAGATACCACCCTA $\begin{array}{llllllllllllllllllllll}G & L & W & V & T & V & Y & Y & G & V & P & V & W & K & D & A & D & T & T & L\end{array}$ TTTTGTGCATCAGATGCTAAGgCATATGATACAGAAGTGCATAATGTCTGGGCTACACAT

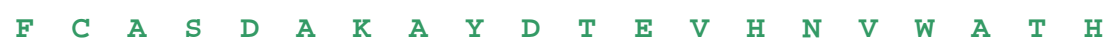
GCCTGTGTACCCACAGACCCCAACCCACAAGAAATGACTTTAATGAATGTAACAGAAAAG

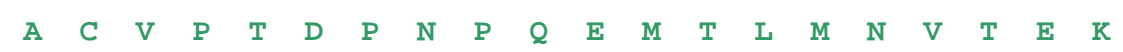
TTTAACATGTGGAAAAATAACATGGTAGAACAAATGCACACAGATATAATCAGTTTATGG $\begin{array}{lllllllllllllllllllll}F & N & M & W & K & N & N & M & V & E & Q & M & H & T & D & I & I & S & L & W\end{array}$ GACCAAAGCCTAAAACCATGTGTAAGCTTAACCCCTCTCTGTGTTACTTTAAATTGCAGA

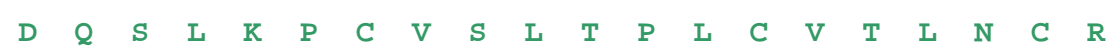
AATGTCACTATTAATGACACTATTAGAAACAGCAGTGTTATTGgTGACATGAAAGAAGAA $\begin{array}{llllllllllllllllllllll} & N & V & T & I & N & D & T & I & R & N & S & S & V & I & G & D & M & K & E & E\end{array}$ GTAACAAATTGCTCTTTCAATATAACCACAGAACTAAGAGATAAGAGACAAAAAGTATAT

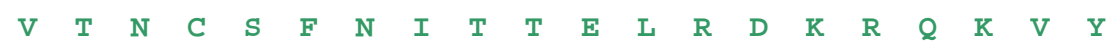
TCACTTTTTTATAAACTTGATGTAGTACAAATTAATCCTGCTGATAAGAATAGTACCCAA $\begin{array}{llllllllllllllllllllll}S & L & F & Y & K & \text { L } & D & V & V & Q & I & N & P & A & D & K & N & S & T & Q\end{array}$ TATAGACTAATAAATTGTAATACCTCAACCATTACACAGGCTTGTCCAAAGGTATCCTTT $\begin{array}{llllllllllllllllllll}Y & R & L & I & N & C & N & T & S & T & I & T & Q & A & C & P & K & V & S & F\end{array}$ GAGCCAATTCCCATACATTATTGTGCTCCAGCTGGTTTTGCGATTCTAAAGTGTAATGAT

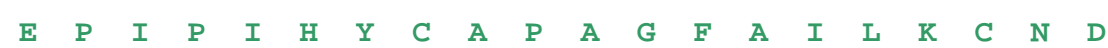




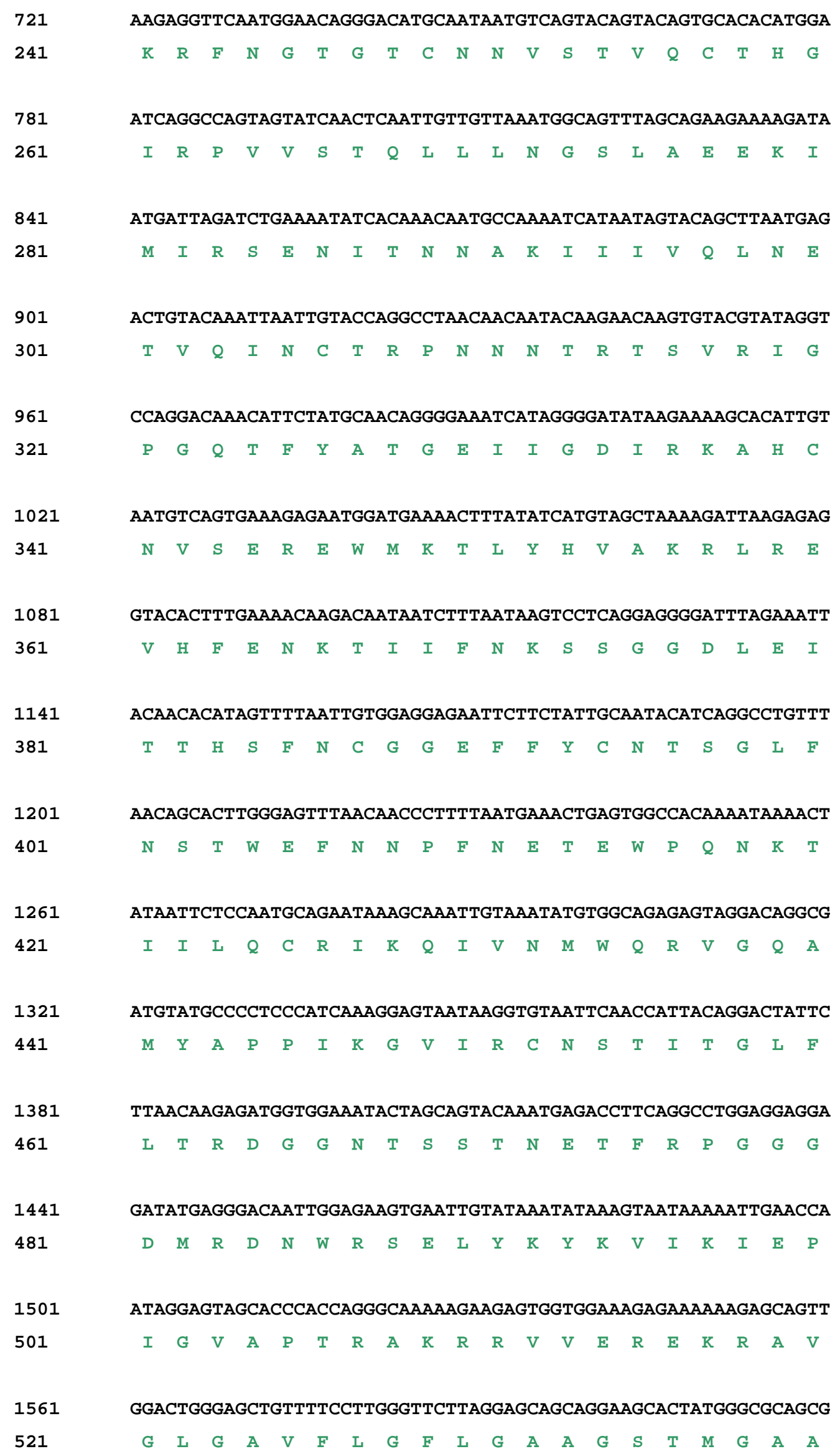




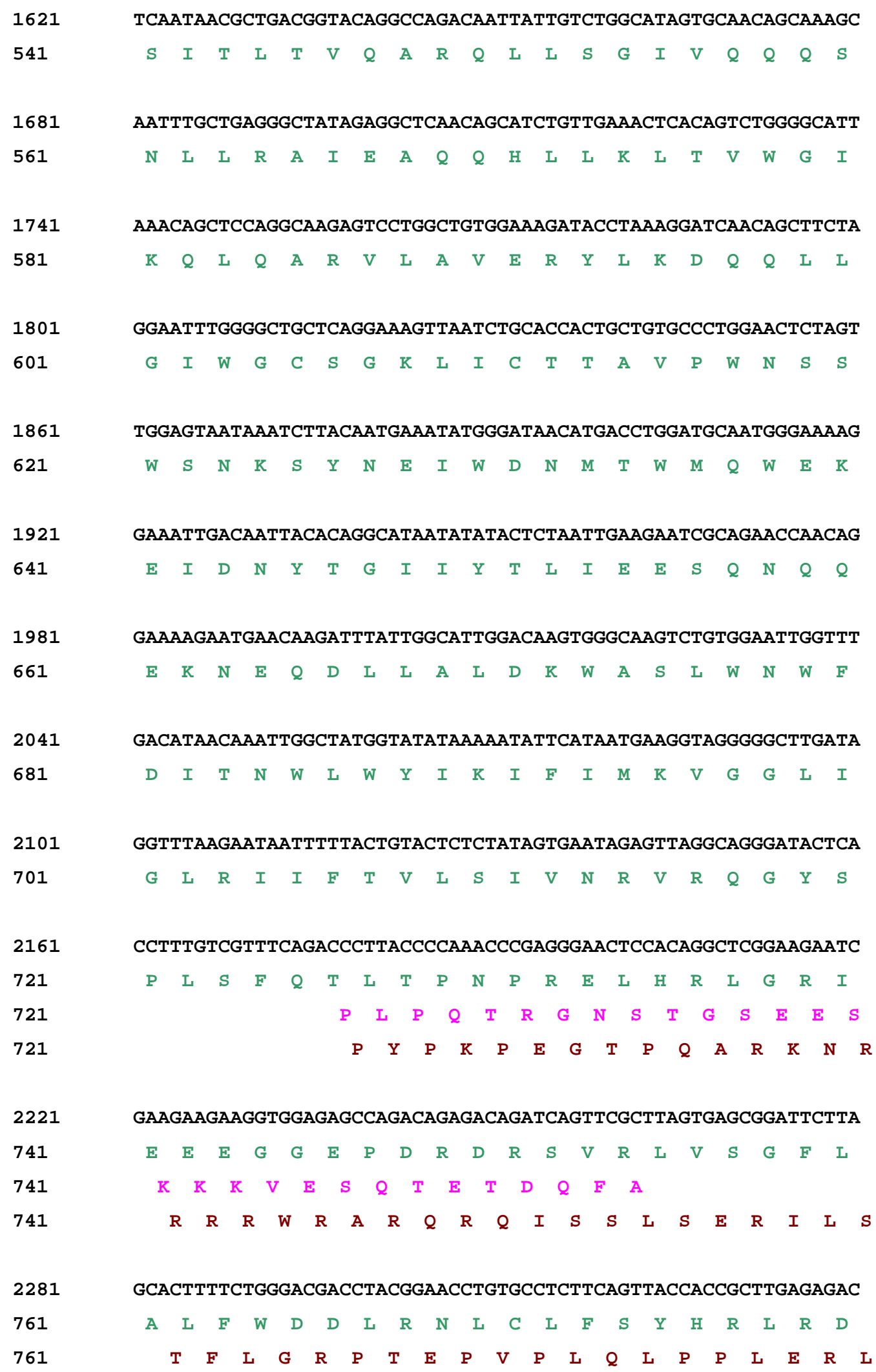




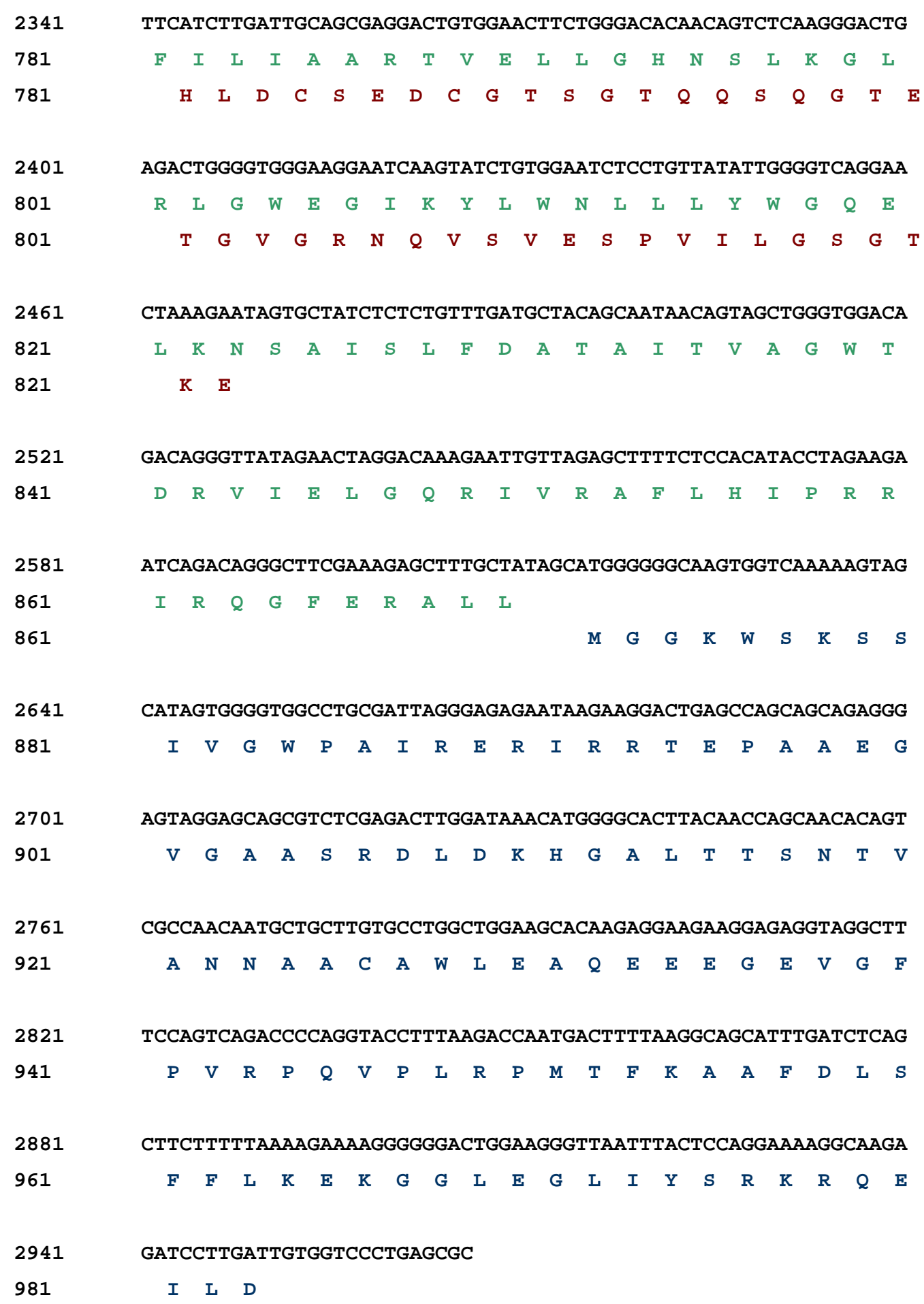


Sample TV 314 from 1235 - 9551 (coordinate relative to HXB2) Nucleotide and amino acid composition

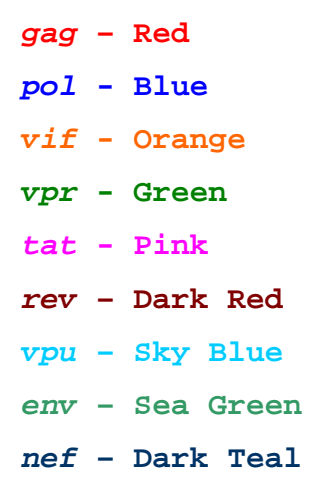

\section{TCAGGACTTTGGATGCATGGGTAAAAGTAATAGAAGAAAAGGCTTTCAGCCCTGAAGTAA} $\begin{array}{llllllllllllllllllll}R & T & L & D & A & W & V & K & V & \text { I } & \text { E } & \text { E } & K & \text { A } & F & \text { S } & \text { P } & \text { E } & \text { V } & \text { I }\end{array}$ TACCCATGTTCTCAGCATTATCAGAAGGAGCCACCCCACAAGATTTAAATATGATGCTGA $\begin{array}{lllllllllllllllllllll}P & M & F & S & A & L & S & E & G & A & T & P & Q & D & L & N & M & M & L & N\end{array}$ ACATAGTGGgGgGACACCAGgCAGCTATGCAAATGTTAAAGgATACCATCAATGAGgAAG $\begin{array}{lllllllllllllllllllll}I & V & G & G & H & Q & A & A & M & Q & M & L & K & D & T & I & N & E & E & A\end{array}$ CTGCAGAATGGgATAGgCTACATCCAGTACATGCAgGgCCAGTTGCACCAGgCCAGATGA $\begin{array}{llllllllllllllllllll}\text { A } & \text { E } & \text { W } & \text { D } & \text { R } & \text { L } & \text { H } & \text { P } & \text { V } & \text { H } & \text { A } & \text { G } & \text { P } & \text { V } & \text { A } & \text { P } & \text { G } & \text { Q } & \text { M } & \mathbf{R}\end{array}$ GAGAACCAAGGGGAAGTGATATAGCAGGAACTACTAGTACCCCTCAAGAACAAATAGCAT

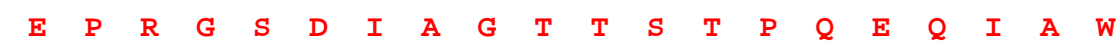
GGATGACAGGCAACCCACCTATCCCAGTGGGAGACATCTATAAAAGATGGATAATCCTAG $\begin{array}{lllllllllllllllllllll}M & T & G & N & P & P & I & P & V & G & D & I & Y & K & R & W & I & I & L & G\end{array}$ GGTTAAATAAAATAGTAAGAATGTATAGCCCTGTTAGCATTTTGGATATAAAACAAGGGC $\begin{array}{llllllllllllllllllllll}\text { L } & N & \text { K } & \text { K } & \text { I } & \text { V } & \text { R } & \text { M } & \text { Y } & \text { S } & \text { P } & \text { V } & \text { S } & \text { I } & \text { L } & \text { D } & \text { I } & K & \text { K } & \text { G } & \text { P }\end{array}$ CAAAAGAACCCTTCAGAGACTATGTAGATAGGTTCTTTAAAACTCTCAGGGCTGAGCAAG $\begin{array}{llllllllllllllllllllll}K & E & P & F & R & D & Y & V & D & R & F & F & K & T & L & R & A & E & Q & A\end{array}$ CTACACAgGAAGTAAAAAATTGGTGACAGAAACATTATTAGTACAAAATGCAAATCCAG $\begin{array}{llllllllllllllllllll}T & Q & E & V & K & N & W & M & T & E & T & L & L & V & Q & N & A & N & P & D\end{array}$ ATTGTAAgTCCATTTTAAGAGCATTAGgACCAGgGGCTACATTAGAAGAAATGATGACAG $\begin{array}{llllllllllllllllllllll}C & K & S & I & L & R & A & L & G & P & G & A & T & L & E & E & M & M & T & A\end{array}$ CATGCCAGgGAGTGgGagaCCTAGCCATAAAGCAAGgGTTTTAGCCGAGGCAATGAGTC
$\begin{array}{lllllllllllllllllllll}C & Q & G & V & G & G & P & S & H & K & A & R & V & L & A & E & A & M & S & Q\end{array}$ 


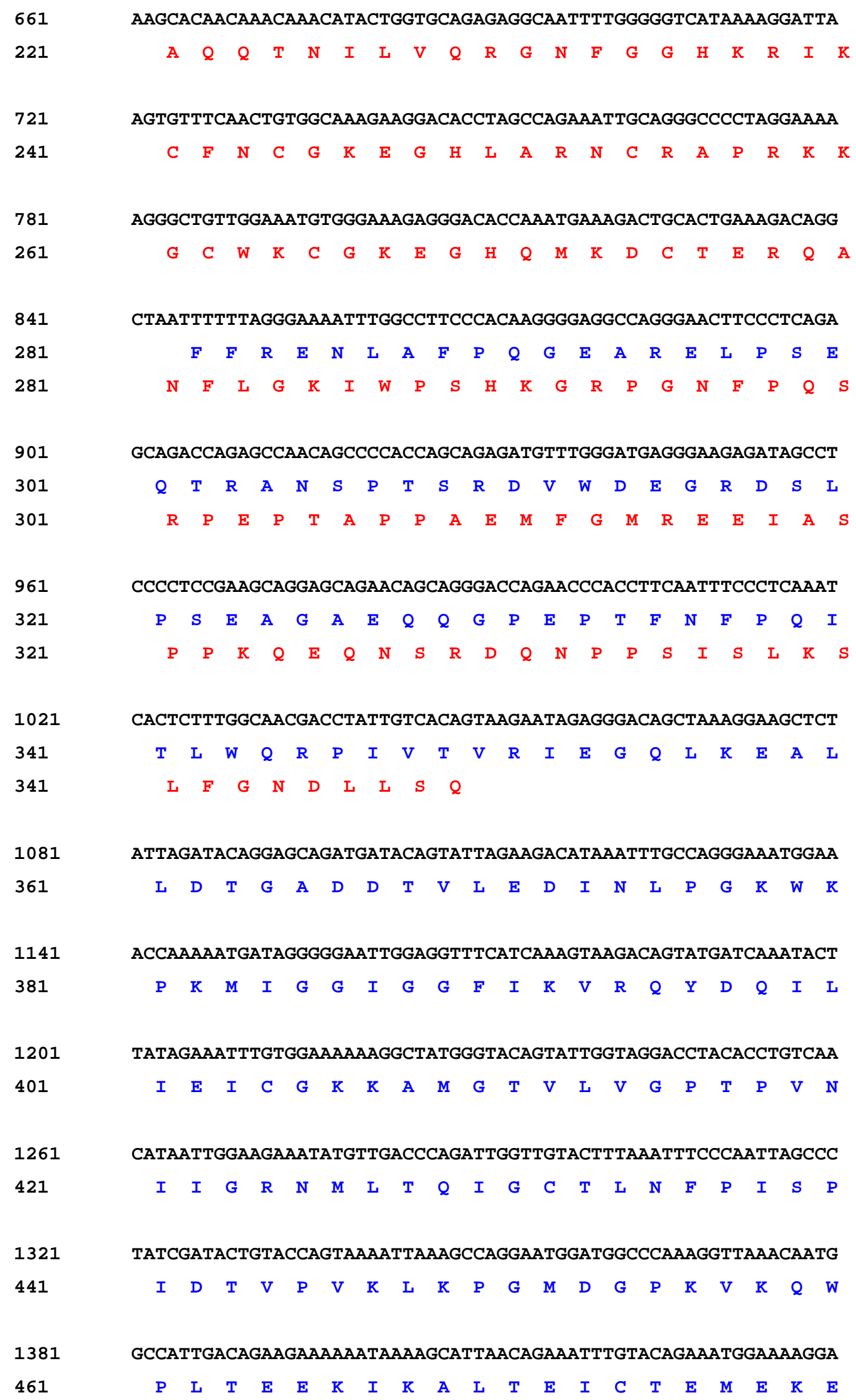

CACTCTTTGGCAACGACCTATTGTCACAGTAAGAATAGAGGGACAGCTAAAGGAAGCTCT $\begin{array}{llllllllllllllllllll}\mathbf{T} & \mathbf{L} & \mathbf{W} & \mathbf{Q} & \mathbf{R} & \mathbf{P} & \mathbf{I} & \mathbf{V} & \mathbf{T} & \mathbf{V} & \mathbf{R} & \mathbf{I} & \mathbf{E} & \mathbf{G} & \mathbf{Q} & \mathbf{L} & \mathrm{K} & \mathrm{E} & \mathbf{A} & \mathbf{L}\end{array}$ 
1441 AGGAAAAATTTCAAAAATTGGGCCTGAAAATCCATACAATACTCCAATATTTGCTATAAA

$\begin{array}{llllllllllllllllllllll}481 & G & K & I & S & K & I & G & P & E & N & P & Y & N & T & P & I & F & A & I & K\end{array}$

1501 GAAAAAGACAGCACTAAATGGAGAAAATTAGTAGATTTCAGAGAGCTCAATAAAAGAAC $\begin{array}{llllllllllllllllllllll}501 & K & K & D & S & T & K & W & R & K & L & V & D & F & R & E & L & N & K & R & T\end{array}$

1561 TCAAGACTTTTGGGAAGTTCAATTAGGAATACCGCATCCAGCGgGCTTAAAAAAGAAAAA $\begin{array}{llllllllllllllllllllll}521 & Q & D & F & W & E & V & Q & \text { L } & \text { G } & \text { I } & \text { P } & \text { H } & \text { P } & \text { A } & \text { G } & \text { L } & \text { K } & \text { K } & \text { K } & \text { K }\end{array}$

1621 ATCAGTAACAGTACTAGATGTGGgGGACGCATATTTTTCAGTTCCCTTAGATGAAAGTT $\begin{array}{lllllllllllllllllllllll}541 & S & V & T & V & \text { L } & \text { D } & \text { V } & \text { G } & \text { D } & \text { A } & \text { Y } & \text { F } & \text { S } & \text { V } & \text { P } & \text { L } & \text { D } & \text { E } & \text { S } & F\end{array}$

1681 TAGAAAGTATACTGCATTCACCATACCTAGTATAAACAATGAGACACCAGGAATCAGGTA $\begin{array}{lllllllllllllllllllll}561 & R & K & Y & \text { T } & \text { A } & \text { F } & \text { T } & \text { I } & \text { P } & \text { S } & \text { I } & \text { N } & \text { N } & \text { E } & \text { T } & \text { P } & \text { G } & \text { I } & \text { R } & \text { Y }\end{array}$

1741 TCAGTACAATGTGCTTCCACAGgGATGGAAAGATCACCAGCAATATTCCAGAGTAGCAT $\begin{array}{llllllllllllllllllllll}581 & Q & Y & N & V & \text { L } & \text { P } & \text { Q } & \text { G } & \text { W } & \text { K } & \text { G } & \text { S } & \text { P } & \text { A } & \text { I } & \text { F } & \text { Q } & \text { S } & \text { S } & \text { M }\end{array}$ 1801 GACAAAAATCTTAGATCCCTTTAGGTCAAAAAATCCAGAACTAATTATCTATCAATACAT $\begin{array}{llllllllllllllllllllll}601 & T & K & I & L & D & P & F & R & S & K & N & P & E & L & I & I & Y & Q & Y & M\end{array}$ 1861 GGATGACTTGTATGTAGgATCTGATTTAGAAATAGgGCAGCATAGAGCAAAAATAGAAGA $\begin{array}{llllllllllllllllllllll}621 & D & D & L & Y & V & G & S & D & L & E & I & G & Q & H & R & A & K & I & E & E\end{array}$ 1921 GTTGAGAGCTCATCTATTAAGCTGGgGATTACTACACCAGACAAAAAGCATCAGAAAGA $\begin{array}{lllllllllllllllllllll}641 & \text { L } & \text { R } & \text { A } & \text { H } & \text { L } & \text { L } & \text { S } & \text { W } & \text { G } & \text { F } & \text { T } & \text { T } & \text { P } & \text { D } & \text { K } & \text { K } & \text { H } & \text { Q } & \text { K } & \text { E }\end{array}$ 1981 GCCTCCATTCCTTTGGATGGGATATGAACTCCATCCTGACAAGTGGACAGTCCAACCTAT $\begin{array}{llllllllllllllllllllll}6 & \text { P } & \text { P } & \text { F } & \text { L } & \text { W } & \text { M } & \text { G } & \text { Y } & \text { E } & \text { L } & \text { H } & \text { P } & \text { D } & \text { K } & \text { W } & \text { T } & \text { V } & \text { Q } & \text { P } & \text { I }\end{array}$ 2041 ACAGCTGCCAGAAAAAGACAGTTGGACTGTCAATGATATACAGAAGCTAGTGGGGAACT $\begin{array}{lllllllllllllllllllll}681 & Q & \text { L } & P & \text { E } & K & \text { D } & \text { S } & \text { W } & \text { T } & \text { V } & \text { N } & \text { D } & \text { I } & \text { Q } & \text { K } & \text { L } & \text { V } & G & \text { K } & \text { L }\end{array}$ 2101 AAATTGGGCAAGTCAGATTTACCCAGGGATTCAAGTAAGACAATTGTGTAAACTCCTCAG $\begin{array}{llllllllllllllllllllll}701 & N & \text { W } & \text { A } & \text { S } & \mathbf{Q} & \text { I } & \text { Y } & \text { P } & \text { G } & \text { I } & \mathbf{Q} & \text { V } & \mathbf{R} & \mathbf{Q} & \mathbf{L} & \mathbf{C} & \mathbf{K} & \mathbf{L} & \mathbf{L} & \mathbf{R}\end{array}$ 2161 GGGAGCCAAAGCACTAACAGATATAGTAACATTGACTGAGGAAGCAGAATTAGAATTGGC 721 $\begin{array}{llllllllllllllllllll}G & A & K & A & \text { L } & \text { T } & \text { D } & \text { I } & \text { V } & \text { T } & \text { L } & \text { T } & \text { E } & \text { E } & \text { A } & \text { E } & \text { L } & \text { E } & \text { L } & \text { A }\end{array}$ AGAGAACAGgGAATTCTAAAAGACCCTGTGCATGgAGTCTATTATGACCCATCAAAAGA 
TGATTTTAATCTGCCACCTATAGTAGCAAAGgAGATAGTAGCCAGCTGTGATAAATGCCA 
AGCAGGAAGATGGCCAGTAAAAACAGTACACACAGACAATGGCAGCAATTTCACCAGTGC

GGTAAGAGAGCAAGCTGAACACCTTAAGACAGCAGTACAAATGGCAGTATTCATTCACAA

GGTTTATTACAGgGACAGCAGAGATCCAATTTGGAAAGGACCAGCAAAACTACTCTGGAA

ACAGGATGAGGATTAGAACATGGCACAGTTTAGTAAAACATCATATGTATGTCTCAAGGA

AAACTAAAGATTGGTCTTATAGACATCACTATGAAAGCAGACATCCAAGAGTAAGTTCAG

AAGTACACATCCCACTAGgGGACGCTAGAATAATAGTAAAAACATATTGGGgTCTGCATA 
ATAACACACAAATAGACCCTGACCTGGCAGACCAACTAATTCATCTGCATTATTTTGAAT

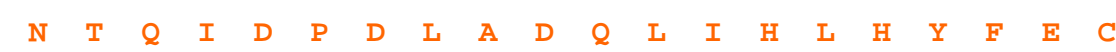
GTTTTTCAGATTCTGCCATAAGGAAAGCCATATTAGGGCGAGTAGTTAACCCTAGGTGTG

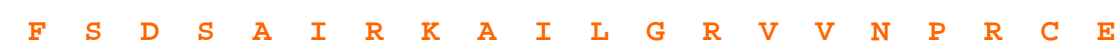
AATATCAAACAGgAAATAAAAAGTAGGATCTCTACAATATTTAGCACTAAAAGCATTAG

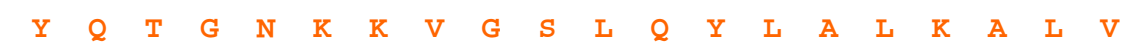

TAGGACCAAAAAAGACAAAGCCACCTTTGCCTAGTGTTAGTAAACTAACAGAGGATAGAT M $\begin{array}{llllllllllllllllllll}G & P & K & K & T & K & P & P & L & P & S & V & S & K & L & T & E & D & R & W\end{array}$ GGAACAAGCCCCAGAAGACCAGGGGCCCAGAGAGAGCCATACAATGAATGGATGTTAGA $\begin{array}{lllllllllllllllllllll} & E & Q & A & P & E & D & Q & G & P & Q & R & E & P & Y & N & E & W & M & L & E\end{array}$ $\begin{array}{llllllllllllllllll}N & K & P & Q & K & T & R & G & P & R & E & S & H & T & M & N & G & C\end{array}$ GCTGTTAGAAGAACTTAAGCATGAAGCTGTTAGACATTTCCCTAGACCATGGCTCCAGGG $\begin{array}{llllllllllllllllllll}\text { L } & \text { L } & \text { E } & \text { E } & \text { L } & \text { K } & \text { H } & \text { E } & \text { A } & \text { V } & \text { R } & \text { H } & \text { F } & \text { P } & \text { R } & \text { P } & \text { W } & \text { L } & Q & G\end{array}$ ACTAGGACAATATATCTACAACACCCATGGGGATACTTGGGAAGGAGTTGAAGCTATTAT $\begin{array}{llllllllllllllllllll}\text { L } & G & Q & Y & I & Y & N & T & H & G & D & T & W & E & G & V & E & A & I & I\end{array}$ AAGAATTTTGCAGCAACTACTGTTTGTTCATTTCAGGATTGGGTGCCAACACAGCAGAAT $\begin{array}{llllllllllllllllllll}R & I & L & Q & Q & \text { L } & \text { L } & F & \text { V } & \text { H } & \text { F } & \text { R } & \text { I } & G & \text { C } & \text { Q } & \text { H } & \text { S } & \text { R } & \text { I }\end{array}$ AGgCATTATTCGAGgGAGAAGAGTCAGAAATGGATCCAGTAGATCCTAACCTAGAGCCCT $\begin{array}{lllllllllllllllll} & G & I & I & R & G & R & R & V & R & N & G & S & S & R & S\end{array}$ GGAACCATCCAGGAAGTCAGCCTACAACTCCTTGTAGCAAGTGTTACTGTAAAGCGTGTT $\begin{array}{llllllllllllllllllll}N & H & P & G & S & Q & P & T & T & P & C & S & K & C & Y & C & K & A & C & C\end{array}$ GCTACCATTGCTTAGTTTGCTTTCAGACCAAAGGCTTAGGCATCTCCTATGGCAGGAAGA M A $\quad \mathbf{G} \quad \mathbf{R}$ $\begin{array}{lllllllllllllllllllll}\text { Y } & \text { H } & \text { C } & \text { L } & \text { V } & \text { C } & \text { F } & \text { Q } & \text { T } & \text { K } & \text { G } & \text { L } & G & \text { I } & \text { S } & \text { Y } & G & R & K & K\end{array}$ AGCGGAGACAGCGACGAGGCACTCCTCACAGCCGTACGGATCATCAAAATCCTGTATCAA

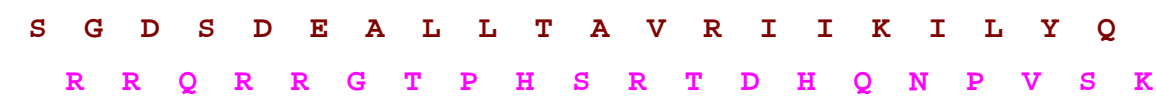
AGCAGTAAGTGTTTATATATGTAATGACCCCTTTAGAAATTAGTGCAATAATAGGATTGA $S$ Q 
GTGGCAATGAGAGTGAGGGGGATACAGATGACTTGGCAGCACTTATTGGGATGGGGAATT

ATGATCTTGGGGATGATTATAATGTGTAGTACTGCAGACAACTTGTGGGTTACTGTTTAC

TATGGGGTACCTGTGTGGAAAGATGCAGAGACCACCCTATTTTGTGCATCAGATGCTAAA

GCATATGAGAAAGAAGTGCATAATGTCTGGGCTACACATGCCTGTGTACCCACAGACCCC

ATGGTAGAGCAGATGCATGCAGATATAATCAGTCTATGGGACCAAAGCCTAAAGCCATGT

GTAAAGCTAACCССTCTCTGTGTAACTTTAAATTGTACCAATGCCAATATCACCTATGTC

AGTACCAACAGCACGAAGGCCTATGTCACTGTCAACGGCACAACGGAAGAAATAAAAAAC

TGCTCTTATAATATGACCACAGAACTAAGgGATAAGAAACAGAAAGTATATTCACTTTTT

TATAGACTTGATGTAGTACAGATTAATAAAAATAATAATAGTAGAGATAATGATAGTGGT 
6481 GTAGCACCATCCAAGgCAAAGAGAAGAGTGGTGGGAAGAGAAAAAGAGCAGTTGGACTG

6541 GGAGCTGTATTCATTGGGTTCTTGGGAGCAGCAGGAAGCACTATGGGCGCGGCGTCAGTG 
TCATTTCAGACCCATACCCCAAACCCAGGGGGACTCGACAGGCCCGAAAGAACAGAAGAA

GAAGGTGGAGTGCAAGGCAGAGACAGATCGATTCGATTAGTCAGCGGATTCTTAGCTCTT 


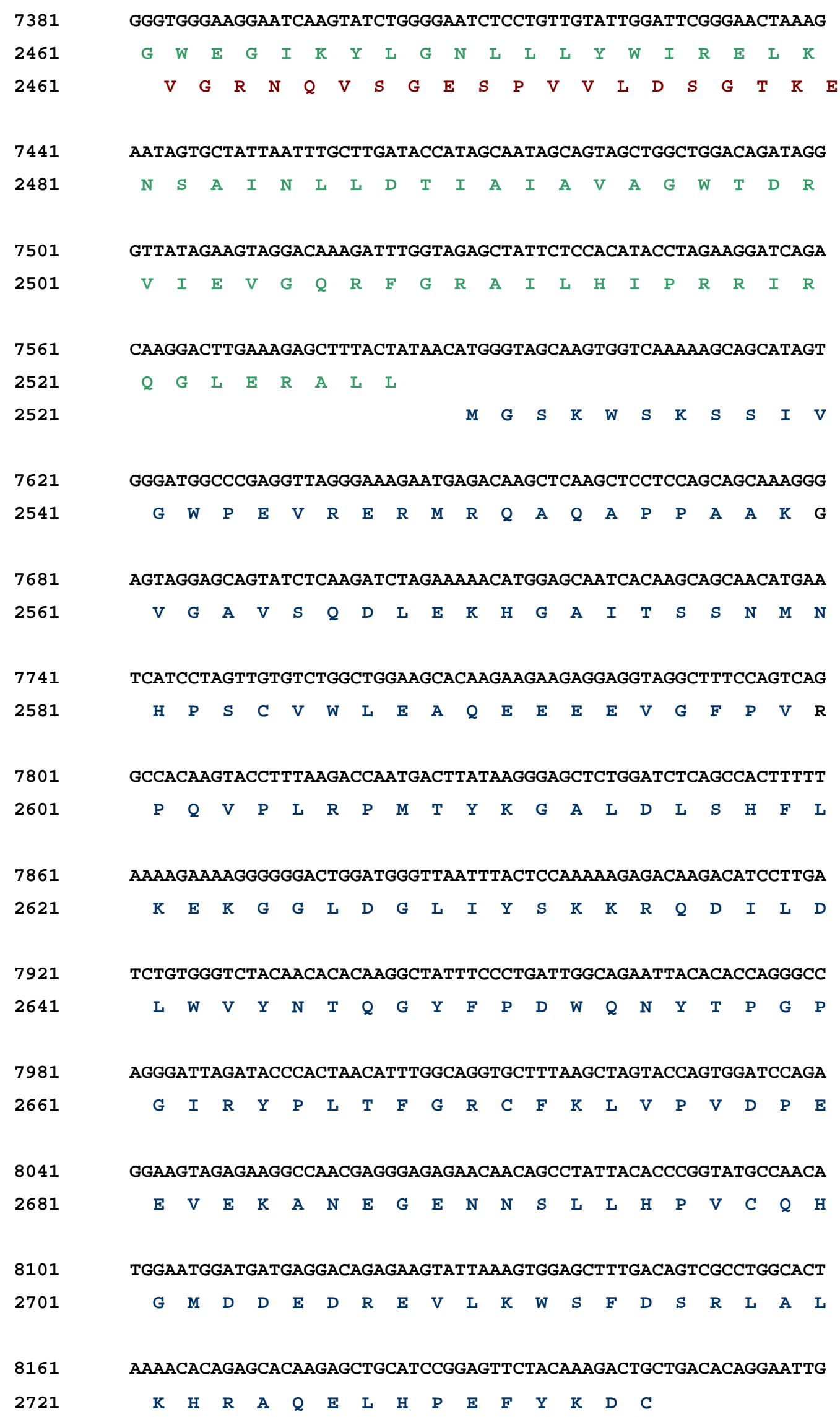

CAAGgACTTGAAAGAGCTTTACTATAACATGgGTAGCAAGTGGTCAAAAAGCAGCATAGT

TCTGTGGGTCTACAACACACAAGGCTATTTCCCTGATTGGCAGAATTACACACCAGGGCC $\begin{array}{lllllllllllllllllllll}\mathbf{L} & \text { W } & \mathbf{V} & \mathbf{Y} & \mathbf{N} & \mathbf{T} & \mathbf{Q} & \mathbf{G} & \mathbf{Y} & \mathbf{F} & \mathbf{P} & \mathbf{D} & \mathbf{W} & \mathbf{Q} & \mathbf{N} & \mathbf{Y} & \mathbf{T} & \mathbf{P} & \mathbf{G} & \mathbf{P}\end{array}$ AGGGATTAGATACCCACTAACATTTGGCAGGTGCTTTAAGCTAGTACCAGTGGATCCAGA $\begin{array}{lllllllllllllllllllll}G & I & R & Y & P & L & T & F & G & R & C & F & K & L & V & P & V & D & P & E\end{array}$ GGAAGTAGAGAAGGCCAACGAGGGAGAGAACAACAGCCTATTACACCCGGTATGCCAACA $\begin{array}{lllllllllllllllllllll} & \text { E } & V & E & K & A & N & E & G & E & N & N & S & L & L & H & P & V & C & Q & H\end{array}$ AAAACACAGAGCACAAGAGCTGCATCCGgAGTTCTACAAAGACTGCTGACACAGGAATTG $\begin{array}{lllllllllllllll}K & H & R & A & Q & E & L & H & P & E & F & Y & K & D & C\end{array}$ 
Sample TV412 from 1246 - 8254 (coordinates relative to HXB2)

Nucleotide and amino acid composition

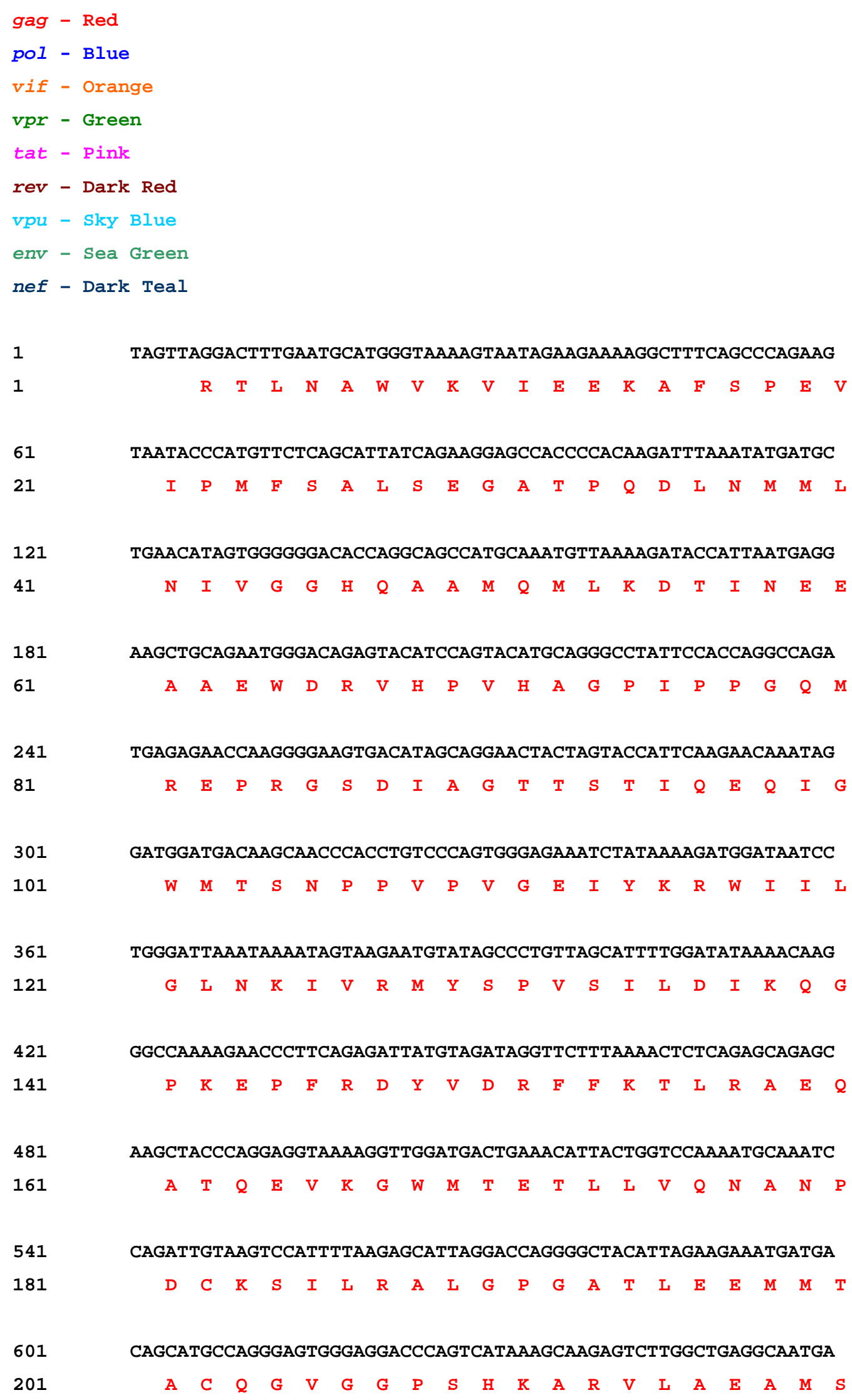




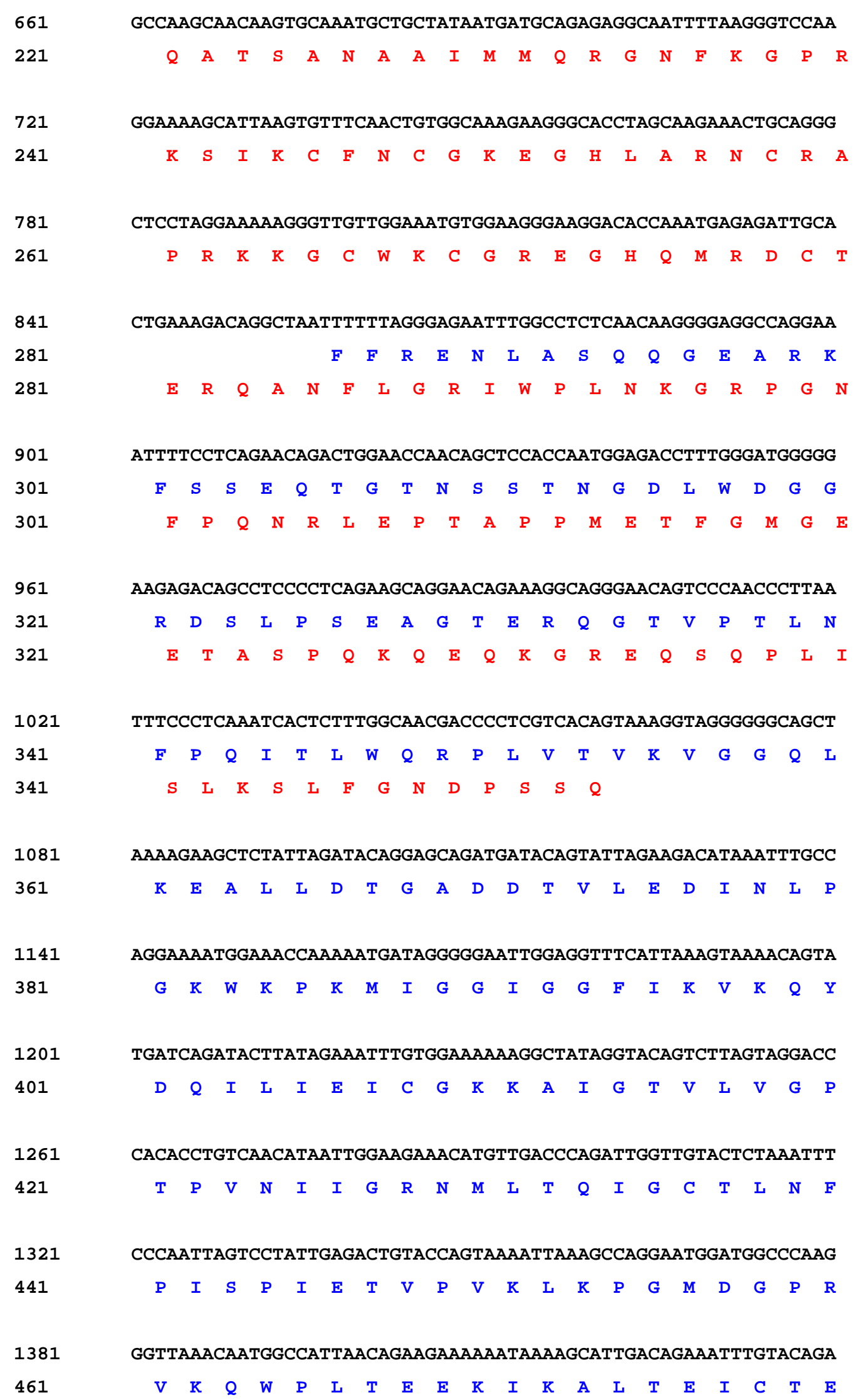

TGATCAGATACTTATAGAAATTTGTGGAAAAAAGGTATAGGTACAGTCTTAGTAGGACC 
AATAGACATAATAGCAACAGACATACAAACTAAAGAACTACAAAAACAAATTACAAAAAT

TCAAAATTTTCGgGTTTATTACAGGGACAGCAGAGATCCAGTTTGGAAAGGACCAGCAAA

TGTGGCAGGTAGACAGGATGAGGATTAAAACATGGAACAGTTTAGTAAAGCATCATATGT

ATGTCTCAAAGAAAGCTAAAGATTGGTTCTATAGACATCATTATGAAAGCAGGCATCCAA

AAgTAAgTTCAgAAgTACACATCCCACTCGgAgAAGCTAGACTGGTAGTAAGAACATATT 
GGAAAAGGAGATATAGCACACAAATAGACCCTGGCCTGGCAGACCAACTAATTCATATAC

CTCCTAGgTGTAATTATCAAGCAGgACATAACAAGGTAGGATCTTTACAATATTTGGCAT

CAGAAGATAGATGGAACGAACCCCAGAGGACCAAGGACCACAGAGGGAGCCATGCAATGA

ATGGACATTAGAGCTTTTAGAGGAGCTCAAGAGTGAAGCTGTTAGACACTTTCCTAGGCC $\begin{array}{llllllllllllllllllll}\text { W } & \text { T } & \text { L } & \text { E } & \text { L } & \text { L } & \text { E } & \text { E } & \text { L } & \text { K } & \text { S } & \text { E } & \text { A } & V & \text { R } & \text { H } & \text { F } & \text { P } & \text { R } & \text { P }\end{array}$ G H ATGGCAGGAAGAAGCGGAAACAGCGACGAAGACCTCCTGAAGGCGGTCAGGCTCATCAAG 
TGGGGAACCATGATTATGGGGATATTAATAATTTGTAGTACTGCAGAAGAAACGTGGGTT

ACTGTCTACTATGGGGTACCTGTGTGGAGAGACGCAGAGACCACCTTATTTTGTGCATCA

GATGCTAAGGCATATGAGACAGAAAAGCATAATGTCTGGGCTACACATGCCTGTGTACCC

ACAGACCCCAGCCCACAAGAAATATATTTGGAAAATGTGACAGAACAGTTTAACATGTGG

AAGCCATGTGTACGGTTAACCCCTCTCTGTGTTACTTTAGAGTGTAGTGACGTCATTAAC

AAAACCAGAGGTACCATCAACCAAACCATAGAACAAAGAATGGAAGGAGAAATAAAAAAC

TGCTCTTACAATATGACCACAGAACTAAGgGATAAGAGACAAAAAGTACAGTCATTATTT

TATAGACTTGATGTAGTAAAAATTAATAAAAATAGTAATAACACAAATACCAGTGAATAT

AGATTAATAAATTGTAATACCTCAGCCATTACACAAGCATGCCCAAAGgTAACCTTTGAG 
GTCAGTAAAAAACAATGGGATGGAATGTTGCAAAAAGTAGCCGACCAATTAAGAACACAT

TTTGGGGAAACAAAACAATAATCTTTGCTAACTCCTCAGGAGGGGACGTACAAATTACA

6481 GGAGTAGCACCCACCAGgGCAAAGAGAAGAGTGGTGGAGAGAGAAAAAAGAGCAGTTGGA

6541 ATAGGAGCTGTTTTCATTGGGTTCTTAGGAGCAGCAGGAAGCACTATGGGCGCGGCGTCA $\begin{array}{lllllllllllllllllllllll}2181 & I & G & A & V & F & I & G & F & L & G & A & A & G & S & T & M & G & A & A & S\end{array}$ 
TTAAGAATAGTTGCTGTGCTTTCTATAATAAATAGAGTTAGgCAGgGATACTCACCTTTG 
7381 GGGTGGGAAGGAATCAAGTATCTGGGGAATCTCCTGTTGT

2461

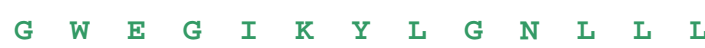

2461

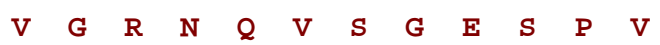




\section{Appendix E}

Table 6.8: jpHMM subtyping results of NFLG's fragments.

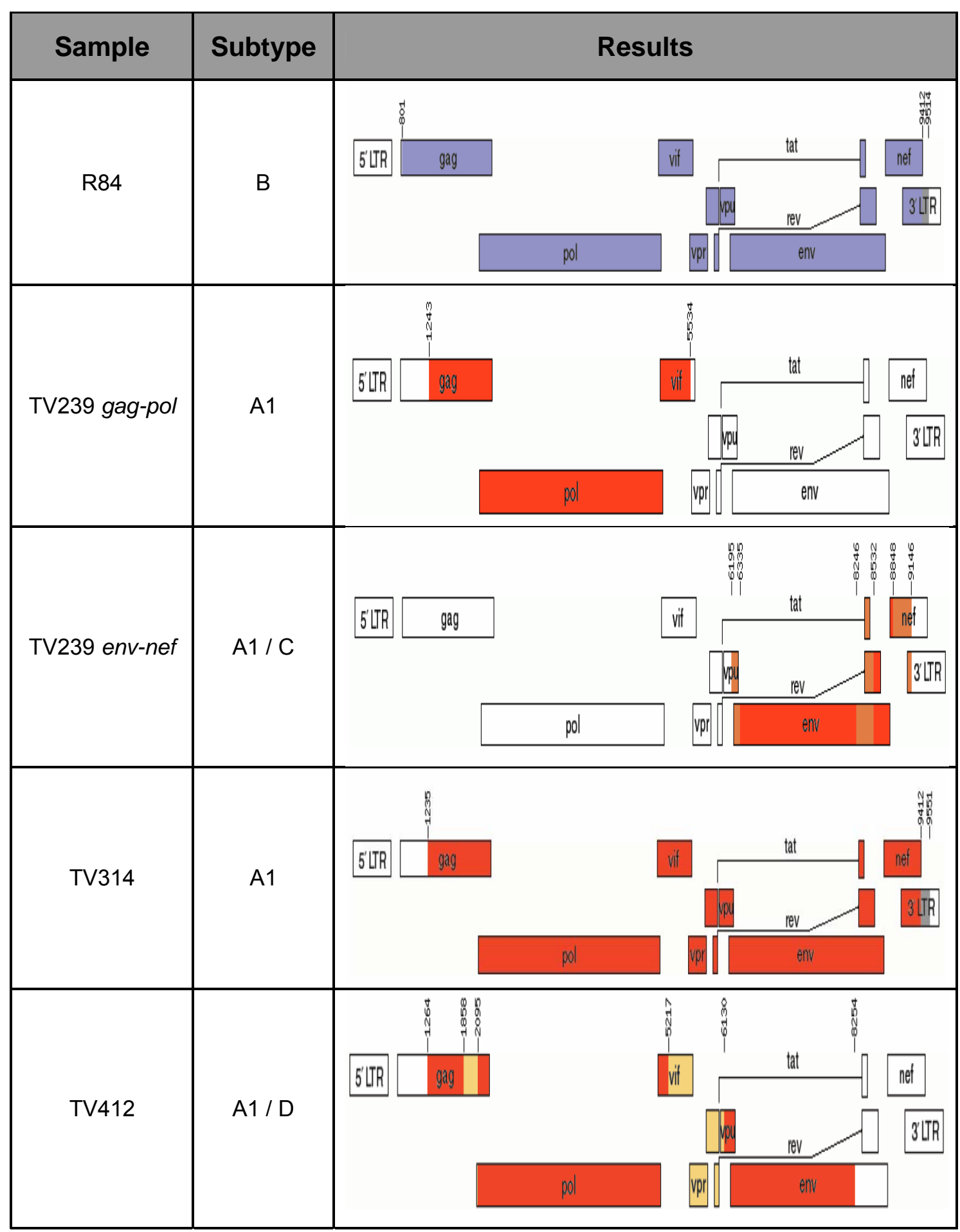




\begin{tabular}{|c|c|c|c|c|c|}
\hline Name & Length & Repoit & Assiginent & Suppoot & Gellome \\
\hline \multirow[t]{2}{*}{ R84 } & \multirow[t]{2}{*}{871060} & \multirow[t]{2}{*}{ Repost } & \multirow[t]{2}{*}{ III-1 Subtype B } & \multirow[t]{2}{*}{100.0} & 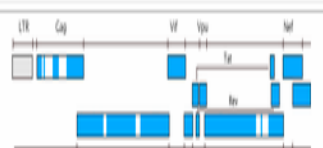 \\
\hline & & & & & 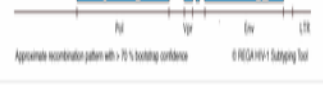 \\
\hline \multirow[t]{2}{*}{ TV239 gage-pol } & \multirow[t]{2}{*}{428760} & \multirow[t]{2}{*}{ Repost } & \multirow[t]{2}{*}{ BIV.1Subtype \&(A1) } & \multirow[t]{2}{*}{100.0} & 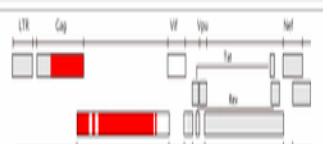 \\
\hline & & & & & 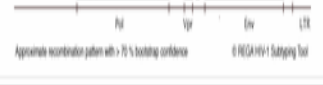 \\
\hline \multirow[t]{2}{*}{ TV239_entr-nef } & \multirow[t]{2}{*}{296600} & \multirow[t]{2}{*}{ Repost } & \multirow[t]{2}{*}{ Check the bootscan } & \multirow[t]{2}{*}{ MA } & 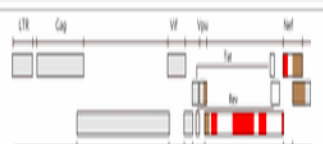 \\
\hline & & & & & 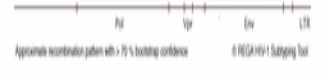 \\
\hline \multirow[t]{2}{*}{ TV314 } & \multirow[t]{2}{*}{834460} & \multirow[t]{2}{*}{ Repost } & \multirow[t]{2}{*}{ 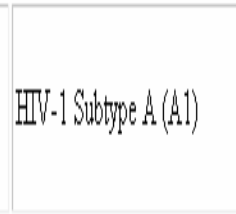 } & \multirow[t]{2}{*}{100.0} & 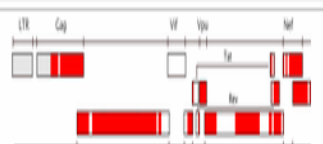 \\
\hline & & & & & 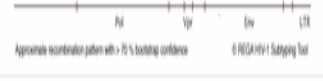 \\
\hline \multirow{2}{*}{ TV412 } & \multirow{2}{*}{$74200 \mathrm{pp}$} & \multirow{2}{*}{ Report } & \multirow{2}{*}{ Cheect the bootscan } & \multirow[b]{2}{*}{$M$} & 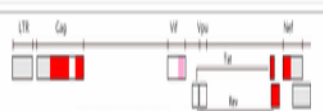 \\
\hline & & & & & $\mathrm{C}_{\mathrm{N}}$ \\
\hline
\end{tabular}

Figure 6.4: REGA subtyping results of NFLG's fragments. 